Modulating learning and memory processes by transcranial magnetic stimulation over the dorsolateral prefrontal cortex

\author{
Ph.D. Thesis
}

Teodóra Vékony

Szeged

2020 


\title{
Modulating learning and memory processes by transcranial magnetic stimulation over the dorsolateral prefrontal cortex
}

\author{
Ph.D. Thesis
}

Teodóra Vékony

Supervisor:

Anita Must, MD Ph.D.

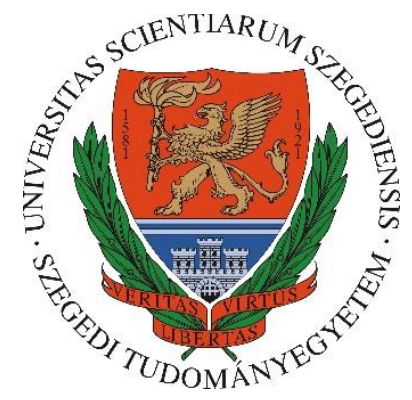

Doctoral School of Clinical Medicine

Department of Neurology

University of Szeged, Hungary 


\section{Original publications directly related to this thesis:}

I. Vékony, T., Németh, V. L., Holczer, A., Kocsis, K., Kincses, Z. T., Vécsei, L., \& Must, A. (2018). Continuous theta-burst stimulation over the dorsolateral prefrontal cortex inhibits improvement on a working memory task. Scientific Reports, 8(1), 1-9. IF = 4.011

II. Ambrus, G. G., Vékony, T.*, Janacsek, K., Trimborn, A. B., Kovács, G., \& Nemeth, D. (2020). When less is more: enhanced statistical learning of non-adjacent dependencies after disruption of bilateral DLPFC. Journal of Memory and Language, 114, 104144. IF = $\mathbf{3 . 8 9 3}$

* = shared first authorship

III. Vékony, T., Marossy, H., Must, A., Vécsei, L., Janacsek, K., \& Nemeth, D. (2020). Speed or accuracy instructions during skill learning do not affect the acquired knowledge. Cerebral Cortex Communications, tgaa041. IF = expected in 2022

Cumulative impact factor of publications related to this thesis: 7.904 
Original publications and review articles not directly related to this thesis:

I. Szegedi-Hallgató, E., Janacsek, K., Vékony, T., Tasi, L. A., Kerepes, L., Hompoth, E. A., Bálint, A., \& Németh, D. (2017). Explicit instructions and consolidation promote rewiring of automatic behaviors in the human mind. Scientific Reports, 7(1), 1-7. IF = 4.122

II. Holczer, A., Németh, V. L., Vékony, T., Vécsei, L., Klivényi, P., \& Must, A. (2020). Noninvasive brain stimulation in Alzheimer's Disease and Mild Cognitive Impairment-A state-ofthe-art review on methodological characteristics and stimulation parameters. Frontiers in Human Neuroscience, 14 . IF $=\mathbf{2 . 6 7 3}$

\section{Preprints or submitted manuscripts not directly related to this thesis:}

I. Vékony, T., Török, L., Pedraza, F., Schipper, K., Pleche, C., Tóth, L, Janacsek, K., \& Nemeth, D. (2020). Retrieval of a well-established skill is resistant to distraction: evidence from an implicit probabilistic sequence learning task. bioRxiv, 849729.

II. Fanuel, L., Pleche, C., Vékony, T., Quentin, R., Janacsek, K., \& Nemeth, D. (2020). The longer the better? General skill but not probabilistic learning improves with the duration of short rest periods. bioRxiv, 090886.

III. Holczer, A., Németh, V. L., Vékony, T., Kocsis, K., Király, A., Kincses, Z. T., Klivényi, P., \& Must, A. (submitted) Bilateral theta-burst stimulation may improve psychomotor speed but does not affect executive functions in major depressive disorder.

IV. Marno, H., Danyi, R., Vékony, T., Janacsek, K., \& Nemeth, D. (submitted) Prompting teaching modulates children's encoding of novel information by facilitating rule-based sequence learning and hindering probability-based statistical learning. 


\section{TABLE OF CONTENTS}

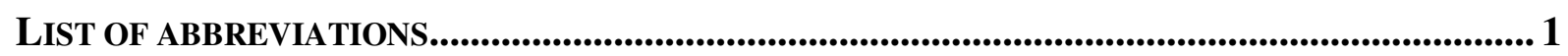

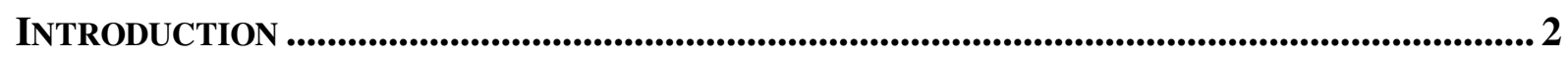

WORKING MEMORY AND ITS NEURAL BACKGROUND .................................................. 2

IMPLICIT PROBABILISTIC LEARNING AND ITS NEURAL BACKGROUND................................... 4

THE RELATIONSHIP BETWEEN IMPLICIT PROBABILISTIC LEARNING AND WORKING MEMORY 5

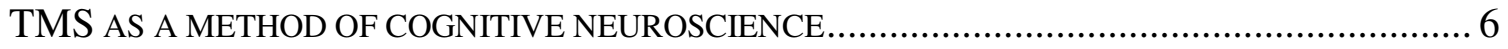

TMS TO EXPLORE WORKING MEMORY PROCESSES ….............................................. 7

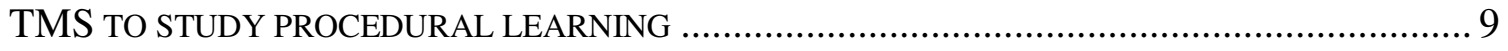

A POTENTIAL METHODOLOGICAL PROBLEM IN THE RESEARCH OF LEARNING AND MEMORY 10

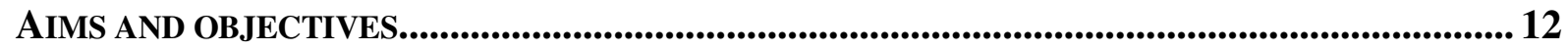

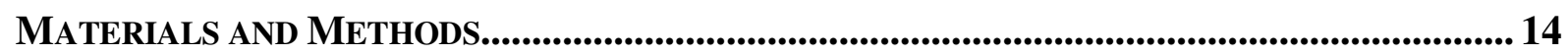

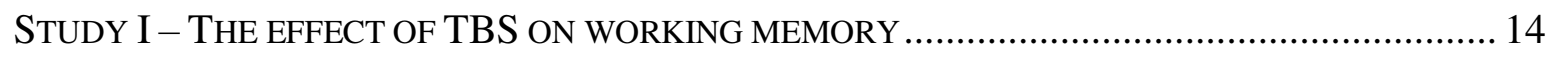

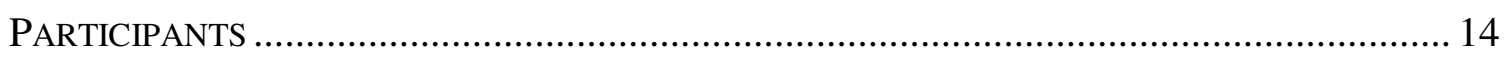

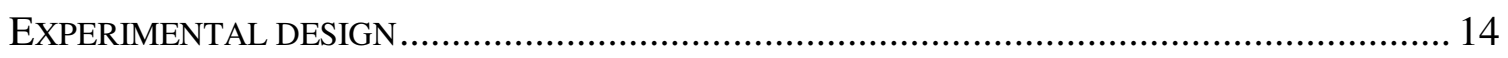

THETA-BURST STIMULATION AND NEURONAVIGATION.................................................... 15

N-BACK TASK AS A MEASURE OF WORKING MEMORY …................................................. 15

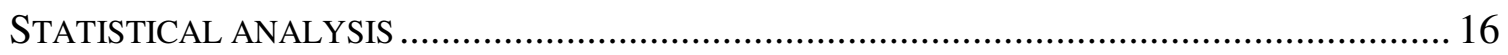

STUDY II - THE EFFECT OF RTMS ON PROBABILISTIC LEARNING ..................................... 17

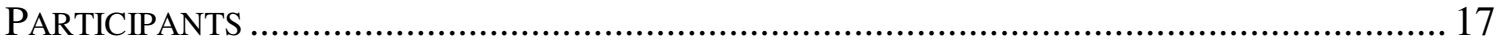

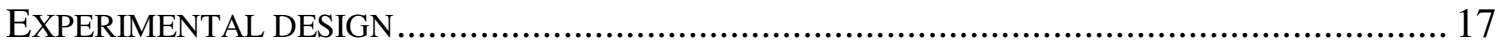

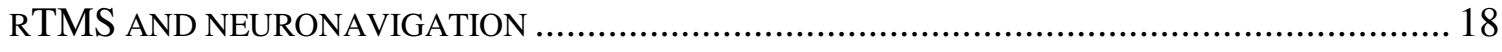

ASRT AS A MEASURE OF IMPLICIT PROBABILISTIC LEARNING ...................................... 19

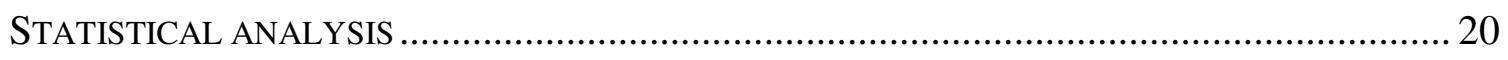

STUDY III - THE EFFECT OF INSTRUCTIONS ON PROBABILISTIC LEARNING ............................ 21 


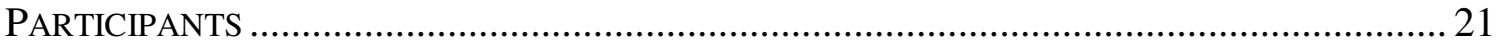

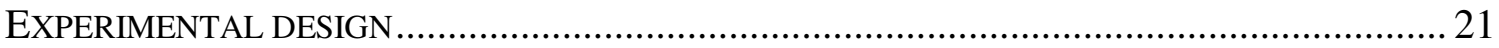

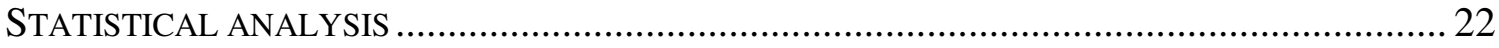

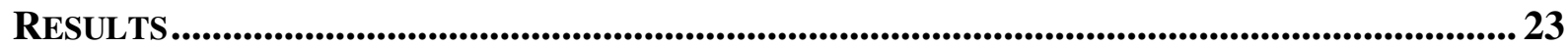

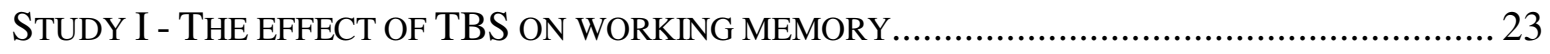

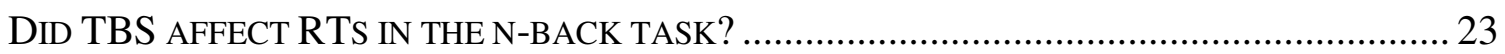

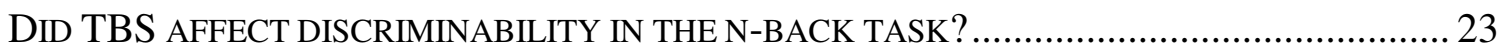

STUDY II - THE EFFECT OF RTMS ON PROBABILISTIC LEARNING ........................................ 24

STUDY III - THE EFFECT OF INSTRUCTIONS ON PROBABILISTIC LEARNING ............................ 25

DID THE INSTRUCTIONS AFFECT GENERAL RTS AND PROBABILISTIC LEARNING IN RT MEASURES IN THE DIFFERENT INSTRUCTION PHASE? ..................................................... 25

DID THE INSTRUCTIONS AFFECT GENERAL ACCURACIES AND PROBABILISTIC LEARNING IN ACCURACY MEASURES IN THE DIFFERENT INSTRUCTION PHASE?

DID THE ACQUIRED KNOWLEDGE DIFFER BETWEEN GROUPS IN THE SAME INSTRUCTION PHASE?

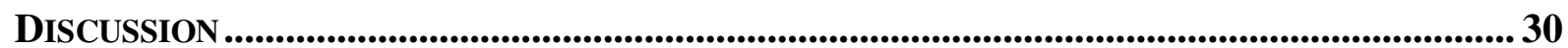

CTBS OVER BOTH DLPFCS HINDERS WORKING MEMORY IMPROVEMENT ......................... 31

DISRUPTING THE DLPFCS LEADS TO IMPROVED IMPLICIT PROBABILISTIC LEARNING ........ 32

THE ROLE OF DLPFC IN WORKING MEMORY AND IMPLICIT PROBABILISTIC LEARNING: WHAT

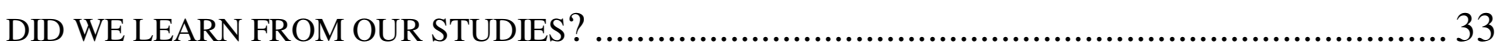

INSTRUCTIONS DO NOT MODIFY PROBABILISTIC REPRESENTATIONS …............................ 35

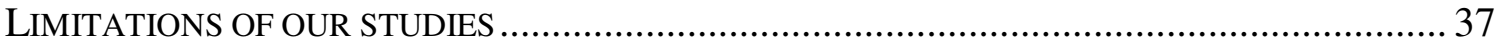

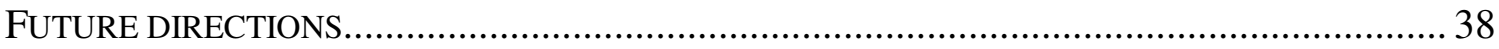

CONCLUSION

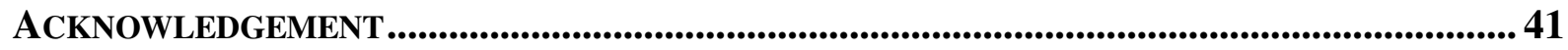

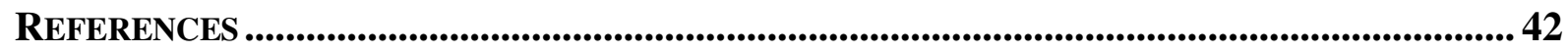




\section{LIST OF ABBREVIATIONS}

\begin{tabular}{|c|c|c|}
\hline ASRT & $=$ & ALteRnAting SERIAL REACTION TIME TASK \\
\hline ANOVA & $=$ & ANALYSIS OF VARIANCE \\
\hline CTBS & $=$ & CONTINUOUS THETA-BURST STIMULATION \\
\hline $\mathbf{D}^{\prime}$ & $=$ & DISCRIMINABILITY INDEX \\
\hline DLPFC & $=$ & DORSOLATERAL PREFRONTAL CORTEX \\
\hline ITBS & $=$ & INTERMITTENT THETA-BURST STIMULATION \\
\hline $\mathbf{M}$ & $=$ & MEAN \\
\hline MSO & $=$ & MAXIMUM STIMULATOR OUTPUT \\
\hline MT & $=$ & MOTOR THRESHOLD \\
\hline RT & $=$ & REACTION TIME \\
\hline RTMS & $=$ & REPETITIVE TRANSCRANIAL MAGNETIC STIMULATION \\
\hline SD & $=$ & STANDARD DEVIATION \\
\hline SE & $=$ & STANDARD ERROR \\
\hline SEM & $=$ & STANDARD ERROR OF THE MEAN \\
\hline SRTT & $=$ & SERIAL REACTION TIME TASK \\
\hline TBS & $=$ & THETA-BURST STIMULATION \\
\hline TMS & $=$ & TRANSCRANIAL MAGNETIC STIMULATION \\
\hline WM & $=$ & WORKING MEMORY \\
\hline
\end{tabular}




\section{INTRODUCTION}

The dorsolateral prefrontal cortex (DLPFC) is a core area within the prefrontal cortex localized in the Brodmann 9/46 regions, which has been associated with a wide range of cognitive functions, including decision making (e.g., Heekeren, Marrett, Ruff, Bandettini, \& Ungerleider, 2006), planning (e.g., Kaller, Rahm, Spreer, Weiller, \& Unterrainer, 2011), executive functions (e.g., Barbey, Colom, \& Grafman, 2013), cognitive control (e.g., MacDonald, Cohen, Stenger, \& Carter, 2000), and also various learning and memory functions, such as working memory processes (WM) (e.g., Owen, McMillan, Laird, \& Bullmore, 2005). The DLPFC was not only connected to conscious and voluntary operations but to more subconscious memory processes such as procedural learning (e.g., Bennett, Madden, Vaidya, Howard, \& Howard, 2011; Simon, Vaidya, Howard, \& Howard, 2012; Stillman et al., 2013).

In the last decades, neuroimaging methods, investigations of patients with a brain lesion, and animal research allowed us to gain insight into the relationship between the DLPFC and related brain networks underlying learning and memory processes. Methods such as local field potentials in animals (e.g., Siegel, Warden, \& Miller, 2009), in-vivo electrical recordings (e.g., Oehrn et al., 2018), electroencephalography (e.g., Kóbor et al., 2018), near-infrared spectroscopy (Sato et al., 2013) or functional magnetic resonance imaging in humans (e.g., Bennett et al., 2011) provided us with a wealth of valuable knowledge on the potential roles of the prefrontal cortex in WM and implicit probabilistic learning. However, their crucial shortcomings limit the conclusions we can derive from them. For instance, neuroimaging methods could only provide correlational evidence between different cognitive functions and brain activity; lesion studies and animal research, on the other hand, are also limited by the fact that their conclusions are not necessarily applicable to the mechanisms of the healthy human brain. On the contrary, non-invasive brain stimulation methods allow us to examine the healthy brain without invasive interventions to drive causal conclusions about brain-behavior relationships. The thesis presents two studies aiming to investigate the role of DLPFC in WM and implicit probabilistic learning with a non-invasive brain stimulation method, namely transcranial magnetic stimulation.

\section{Working memory and its neural background}

WM is a system responsible for temporarily storing, processing, and manipulating goalrelevant information (Baddeley, 1986). In other words, this system holds the stimuli „online” 
for a short period, to then retain or manipulate them with a short delay. This fundamental set of processes is crucial for many higher-level cognitive functions such as complex problem solving, language comprehension, long-term learning, which are essential for the successful management of our daily activities, and to pursue an adaptive behavior (Baddeley, 1992). Thus, it is not surprising that impaired WM is a common cognitive dysfunction in several psychiatric disorders such as schizophrenia, major depression, bipolar disorder (Trivedi, 2006) or neurological conditions such as sclerosis multiplex (DeSousa, Albert, \& Kalman, 2002), mild cognitive impairment, Alzheimer's Disease or Huntington's Disease (Kirova, Bays, \& Lagalwar, 2015; Stout et al., 2011).

One of the most commonly used methods to evaluate WM performance is the n-back task (Kirchner, 1958). In the classic version of this task, visual stimuli (typically letters or numbers) are presented sequentially to the participants. The individuals have to decide whether the currently presented stimulus is the same as the stimulus presented $n$ trials earlier. Thus, the 1-back version (when the decision has to be made in the light of the trial presented one trial earlier) is less demanding for WM, but the 2- and 3-back versions require to store and monitor multiple pieces of information online. They also require maintaining recently processed and potentially relevant, but also rejecting processed and potentially non-relevant stimuli. Moreover, they entail making continuous comparisons between items to identify target stimuli. Thus, the n-back task allows us to evaluate WM performance with increasing cognitive and processing demand.

Neuroimaging techniques have provided us with the ability to study the underlying neural background of WM. Possibly the most extensive meta-analysis of functional neuroimaging studies using the n-back task found task-related activity in many brain regions, including the lateral and medial posterior parietal cortex, the lateral premotor cortex, the rostral prefrontal cortex, the dorsal cingulate, the medial premotor cortex, or the ventrolateral prefrontal cortex. However, the most prominent activity was shown within the DLPFC (Owen et al., 2005). The authors concluded that these regions of the prefrontal cortex could be mainly involved in the reorganization of information into regular or higher-level chunks, i.e., the strategic control of WM processes. Although the involvement of the DLPFC in WM is welldocumented (Owen et al., 2005), recent studies have questioned the necessity of DLPFC for smooth WM-related cognitive operations (Barbey, Koenigs, \& Grafman, 2013; Mackey, Devinsky, Doyle, Meager, \& Curtis, 2016). Apart from the frontal and parietal areas, connections between the striatal regions and the dorsal prefrontal cortex were also associated 
with performance on WM tasks, as these connections are involved in the manipulation of information (Riley, Moore, Cramer, \& Lin, 2011), as well as in the update and selection of representations based on their adaptive value (Scimeca \& Badre, 2012).

\section{Implicit probabilistic learning and its neural background}

The procedural memory system underlies a wide range of cognitive functions, including the (implicit) acquisition of sequences (Squire, 2004). Fronto-striatal connections also support these memory functions (Bennett et al., 2011; Doyon et al., 2009; Poldrack et al., 2005), but contrary to WM, these types of learning support more long-term knowledge formation than momentary storage of information. It is a fundamental mechanism of the brain that underlies our ability to obtain social, cognitive, and motor skills by enabling us to extract the relevant regularities and patterns from the environment (Kaufman et al., 2010; Lieberman, 2000). Surprising, contrary to WM, procedural learning can be entirely or partly intact in psychiatric disorders such as borderline personality disorder (Unoka et al., 2017), neurodevelopmental disorders such as Tourette syndrome, Attention Deficit Hyperactivity Disorder, or autism (Nemeth et al., 2010; Takács et al., 2018, 2017) or neurological conditions such as Huntington's Disease (Schneider et al., 2010).

Implicit learning of sequential regularities can be measured by exposing individuals to a sequence of stimuli and instruct them to follow their appearance. The sequence can be a fixed, repeating sequence (deterministic sequence learning) contrasted to a series of random elements (e.g., Serial Reaction Time Task (Nissen \& Bullemer, 1987)). However, in such deterministic sequence learning tasks, explicit awareness about the regularities can emerge (Cohen \& Poldrack, 2008; Song, Howard, \& Howard, 2007). As a result of the deterministic structure, these tasks measure simple, adjacent regularities (Remillard, 2008). Alternatively, sequence learning can be measured by presenting elements or sequences with higher probability than others (probabilistic sequence learning). This type of learning can be termed as statistical learning as well, as it requires the acquisition of statistical regularities of the series of stimuli, i.e., the probabilities of the appearance of the different items. One particular method to measure probabilistic sequence learning is the Alternating Serial Reaction Time (ASRT) task, where pattern and random elements are alternating (Howard \& Howard, 1997). Here, probabilistic/statistical learning is measured by contrasting the responses to low vs. highprobability elements. This structure is less likely to evoke explicit awareness, and it can also separate general skill improvements (i.e., the continuous improvement on the task due to 
practice) from sequence-specific learning (i.e., the higher sensitivity to more probable events) (Howard \& Howard, 1997; Song et al., 2007).

Studies investigating the neural basis of picking up probabilistic regularities from the environment emphasize the role of fronto-striatal networks. Subcortical structures in the basal ganglia, such as the striatum (caudate nucleus), and the globus pallidus have pivoting roles in probabilistic learning, and also the hippocampal activity has emerged in several studies (Bennett et al., 2011; Gheysen, Van Opstal, Roggeman, Van Waelvelde, \& Fias, 2011; Janacsek et al., 2020; Rieckmann, Fischer, \& Bäckman, 2010; Simon et al., 2012). The role of the DLPFC was claimed controversial in this type of learning, as DLPFC-related activity is not consistently found in studies assessing probabilistic learning (Bo, Peltier, Noll, \& Seidler, 2011; Fletcher et al., 2005; Munoz \& Everling, 2004; Rieckmann et al., 2010; Röttger, Haider, Zhao, \& Gaschler, 2017; Schwarb \& Schumacher, 2009). However, a functional connectivity index between the caudate and medial temporal regions revealed a positive correlation with probabilistic learning (Stillman et al., 2013). The integrity of the neural tracts between the hippocampus and the DLPFC was also related to probabilistic learning performance, and similar results were found regarding the tracts between the caudate nucleus and the DLPFC (Bennett et al., 2011). These results indicate a potential mediating role of the DLPFC between other areas involved in probabilistic learning.

\section{The relationship between implicit probabilistic learning and working memory}

Although WM and implicit probabilistic and sequence learning have partly shared brain networks (i.e., they both rely at least partly on the fronto-striatal networks), their relationship is a subject of continuous debate in the literature (Janacsek \& Nemeth, 2013, 2015; Martini, Sachse, Furtner, \& Gaschler, 2015). It seems intuitive that higher WM capacity enables us to maintain the sequence elements continuously, and thereby, it helps us learn regularities (Frensch \& Miner, 1994). However, the majority of studies investigating the relationship between WM and implicit sequence learning found no connections between the two processes (for review, see Janacsek \& Nemeth, 2013). It was suggested that better WM performance is more related to better explicit sequence learning and general skill improvements than to better implicit sequence learning. Moreover, recent findings emphasize that reducing the engagement of DLPFC (a structure highly involved in WM) can even lead to improved implicit probabilistic sequence learning (Nemeth, Janacsek, Polner, \& Kovacs, 2013; Virag et al., 2015). Such a relationship might be explained by the fact that WM supports the more controlled forms of 
learning mechanisms which - although they do not cover exactly the same mechanisms - are known as declarative memory system (e.g., Scimeca \& Badre, 2012), goal-directed learning (e.g., Eppinger, Walter, Heekeren, \& Li, 2013), or model-based learning (e.g., Otto, Raio, Chiang, Phelps, \& Daw, 2013).

\section{TMS as a method of cognitive neuroscience}

In the last decades, a significant amount of knowledge has been accumulated on the neural background of WM and implicit sequence learning, and also on their relationship. However, neuroimaging and electrophysiological techniques could only provide correlational evidence to support the roles of distinct brain areas/networks in memory processes and do not provide evidence for the causal role of specific areas in these functions. We might overcome this limitation by the use of non-invasive brain stimulation methods. These methods have been developed to temporarily enhance or decrease the excitability of different areas of the brain through the intact scalp (Barker, Jalinous, \& Freeston, 1985; Merton \& Morton, 1980). Following the first successful applications of these methods to influence the excitability of the human motor cortex, non-invasive brain stimulation methods became widely used neuroscientific research tools to study almost every aspect of the human brain, including cognition (Demeter, 2016).

Two major types of non-invasive brain stimulation methods can be distinguished. Transcranial electrical stimulation methods (such as transcranial direct current stimulation, transcranial random noise stimulation, or transcranial alternating current stimulation) operate with weak electrical stimulation through the scalp (Paulus, 2011). Transcranial magnetic stimulation (TMS) methods, on the other hand, work by creating an intense magnetic field via a wire coil that induces a current flow in the targeted cortical tissue (i.e., under the coil). Therefore, neural firing and changes in cortical excitability are elicited with a precision of tens of millimeters (Deng, Lisanby, \& Peterchev, 2013; Hallett, 2000). TMS can be applied as single-pulse (Barker et al., 1985), paired-pulse stimulation (Kujirai et al., 1993), or repetitively, which is known as repetitive transcranial magnetic stimulation (rTMS). The rTMS can modulate cortical activity beyond the duration of the stimulation (Klomjai, Katz, \& LackmyVallée, 2015), which makes it an ideal tool to study cognitive processes such as learning and memory. Moreover, the delivery of rTMS induces activity changes not only in the targeted brain regions, but in distant, interconnected brain areas (Eldaief, Halko, Buckner, \& Pascual-Leone, 2011). However, the actual mechanisms behind the aftereffects of TMS are still not clear. It 
may exert its value by altering the level of various neurotransmitters or cerebral blood flow (Noda et al., 2015). Induced changes in cortical activity beyond the termination of the stimulation suggest a mechanism similar to long-term potentiation and long-term depressionlike plasticity (Klomjai et al., 2015; Luber \& Lisanby, 2014).

So far, several protocols of rTMS have been developed, which have distinct effects on the brain's excitability. The aftereffects of the rTMS are mainly determined by the frequency of the stimulation, the duration of the stimulation period, and the pattern of the application (Klomjai et al., 2015). In general, rTMS applied with $1 \mathrm{~Hz}$ or lower frequencies (low-frequency stimulation) is considered to have inhibitory effects, i.e., inhibition of neural firing. In contrast, stimulation with $5 \mathrm{~Hz}$ or higher (high-frequency stimulation) leads to excitatory effects, i.e., neural depolarization in the underlying brain tissue. As for the duration, typically, longer duration induces longer-lasting effects (Peinemann et al., 2004). However, especially in cognitive domains, other factors also determine the outcome of the stimulation, such as the baseline activity of the target area (Sandrini, Umiltà, \& Rusconi, 2011; Silvanto, Cattaneo, Battelli, \& Pascual-Leone, 2008).

A more recently developed alternative to rTMS is theta-burst stimulation (TBS) (Huang, Edwards, Rounis, Bhatia, \& Rothwell, 2005). TBS consists of gamma frequency trains of burst (typically $50 \mathrm{~Hz}$ ) applied at a theta rhythm; thus, it mimics the theta-gamma coupling which is involved in memory processes (Düzel, Penny, \& Burgess, 2010; Lisman \& Jensen, 2013). One of the two widely-used forms of TBS is continuous TBS (cTBS) that applies TBS continuously for a duration of ca. $40 \mathrm{~s}$. The other often used protocol is the intermittent TBS (iTBS), which applies TBS for $2 \mathrm{~s}$ every $10 \mathrm{~s}$ (typically applied for $190 \mathrm{~s}$ ). The cTBS protocol was found to exert inhibitory, while the iTBS protocol excitatory effects on the human motor cortex (Huang et al., 2005). The advantages of TBS over rTMS include the shorter stimulation periods and the seemingly more prolonged aftereffects (Nyffeler et al., 2006).

\section{TMS to explore working memory processes}

Since the development of TMS protocols to stimulate the human brain, a lot of studies aimed to gather causal evidence for the involvement of specific regions/networks in learning and memory. rTMS was found to effectively modulate various memory processes, such as episodic (Sandrini, Cappa, Rossi, Rossini, \& Miniussi, 2003) or prospective memory (Bisiacchi, Cona, Schiff, \& Basso, 2011). Due to its involvement in WM performance, several 
studies have targeted the DLPFC to modulate WM-related processes (Brunoni \& Vanderhasselt, 2014), and many of them reported enhanced WM performance measured by the n-back task (Bagherzadeh, Khorrami, Zarrindast, Shariat, \& Pantazis, 2016; Bakulin et al., 2020; Weigand, Grimm, et al., 2013). However, most of them applied high-frequency stimulation, and much less is known about the effect of low-frequency rTMS on WM. Moreover, the results became contradictory in the last few years: multiple studies and metaanalyses failed to find a significant effect of neither low- or high-frequency rTMS on WM performance (Gaudeau-Bosma et al., 2013; Guse et al., 2013; Patel, Silla, Pierce, Theule, \& Girard, 2020). Thus, rTMS might not always be capable of influencing WM-related processes.

In the last few years, studies have begun to explore the effects of TBS on WM performance. It is a promising tool to influence WM, as it operates with theta-gamma coupling, which is fundamental for WM-related processes (Lisman, 2010). Behavioral improvements in the n-back task along with increased theta weighted phase-lag index between fronto-parietal regions and increased parietal gamma power was found following facilitatory iTBS (Hoy et al., 2016). Enhanced amplitudes of TMS-evoked event-related potentials and increased theta and gamma power were also described following TBS (Chung, Rogasch, Hoy, \& Fitzgerald, 2018; Chung, Rogasch, et al., 2017); however, without substantial behavioral improvements. On the contrary, cTBS applied over the DLPFC was reported to impair WM (Lee \& D’Esposito, 2012; Schicktanz et al., 2015), along with exerting a decreasing effect on theta power (Chung, Lewis, et al., 2017). Given these inconsistencies, direct comparisons of the effect of cTBS and iTBS protocols with the same stimulation parameters on WM are warranted.

A recent study found decreased WM performance following both cTBS and iTBS, as reflected by a lack of practice effects in reaction times, as well as a disruptive effect of cTBS on task accuracy (Viejo-Sobera et al., 2017). This finding suggests that cTBS might also disrupt our ability to improve in a WM task with practice. So far, practice effects were mostly considered a confounding factor in cognitive measurements. However, practice-related changes can also yield useful information about the underlying mechanism of cognitive performance (Duff et al., 2007; Duff, Callister, Dennett, \& Tometich, 2012) as it is a tool to measure cognitive plasticity (Calero \& Navarro, 2004). Whether TMS can manipulate the ability to learn task-specific knowledge can reveal information about brain plasticity, which is a crucial mechanism for learning and memory (Tse et al., 2018; Vallence et al., 2015). 
Most of the studies mentioned above applied TBS over the left DLPFC only, leaving the effects of TBS over the right DLPFC as an open question. It was found that cTBS over the left but not right DLPFC induces bilateral blood oxygenation changes in the prefrontal area (Tupak et al., 2013), and dopamine release is enhanced only following stimulation over the left DLPFC (Ko et al., 2008). The behavioral effects on WM performance over the left and right DLPFC are yet to be empirically tested. TMS studies found that verbal WM can be modified by stimulation over the left but not the right DLPFC (Mull \& Seyal, 2001; Shields, Mock, Devier, \& Foundas, 2017). Others claimed that rTMS effectively modulates WM equally over the left and the right DLPFC (Hamidi, Tononi, \& Postle, 2009; Hulst et al., 2017; Mottaghy, Döring, Müller-Gärtner, Töpper, \& Krause, 2002; Mottaghy et al., 2000; Preston, Anderson, Silva, Goldberg, \& Wassermann, 2010). It should be noted that, in some cases, the effects of rTMS over the right DLPFC were related to WM tasks containing spatial (Fried, Rushmore, Moss, Valero-Cabré, \& Pascual-Leone, 2014; Sandrini, Rossini, \& Miniussi, 2008) or negatively valenced stimuli (Weigand, Grimm, et al., 2013; Weigand, Richtermeier, et al., 2013).

Taken together, the effects of TMS on WM performance still raises a number of questions. The efficacy of rTMS over the DLPFC to modulate WM performance has been questioned lately (Patel et al., 2020), although many studies did find effects (Bagherzadeh et al., 2016; Bakulin et al., 2020; Weigand, Grimm, et al., 2013). TBS, on the other hand, is an alternative form of rTMS, which can be more useful to modulate cognitive performance. It might be exceptionally capable of modulating $\mathrm{WM}$ as it operates with the theta-gamma coupling known to be involved in memory processes (Lisman \& Jensen, 2013). Although previous studies have suggested the capability of TBS to modify WM processes (Chung et al., 2018; Chung, Rogasch, et al., 2017; Hoy et al., 2016; Viejo-Sobera et al., 2017), there are inconsistencies in the results. It is not clear how the effects of TBS over the left and right DLPFC compare to each other, and also whether iTBS and cTBS have the opposite effect on WM performance. Therefore, one of our studies aimed to further investigate the effect of TBS on WM-related processes.

\section{TMS to study procedural learning}

Contrary to the studies on WM, very few TMS studies were conducted to reveal the causal role of DLPFC in procedural learning. An early rTMS study revealed that $5 \mathrm{~Hz}$ rTMS over the DLPFC during a deterministic serial reaction time task (SRTT) impairs online learning 
(Pascual-Leone, Wassermann, Grafman, \& Hallett, 1996). Inhibitory cTBS over the DLPFC following practice on SRTT led to better sequence knowledge after an 8-hour consolidation period (Galea, Albert, Ditye, \& Miall, 2010). A similar stimulation before learning also resulted in increased learning performance on phonological sequences (Smalle, Panouilleres, Szmalec, \& Möttönen, 2017). Contrary to these results, DLPFC stimulation was found to lead to null effects on a deterministic learning task that required the use of only one hand (Savic, Cazzoli, Müri, \& Meier, 2017). This result raises the possibility that interhemispheric compensation might obscure the effects of the stimulation. More importantly, these studies used deterministic sequences with adjacent regularities and not probabilistic sequences with non-adjacent regularities (Remillard, 2008). Thus, the role of the DLPFC in this complex form of implicit probabilistic learning remains elusive.

To sum up, studies so far suggest that inhibitory stimulation of the DLPFC might lead to increased performance on implicit learning tasks. This is in line with the competition theory that posits that distinct memory processes can compete with each other (Poldrack \& Packard, 2003). However, we do not know whether the stimulation of the DLPFC can modulate the acquisition of the ecologically more valid, non-adjacent statistical regularities. Moreover, studies so far aimed to test the knowledge at a single time point; however, it is still not revealed how the effects of TMS on procedural learning develop over time. Our second study aims to address these issues. Still, it also raised questions about some more general methodological aspects of measurement, potentially affecting the interpretation of learning and memory studies.

\section{A potential methodological problem in the research of learning and memory}

In cognitive neuroscience, learning and memory functions are typically measured at a single time point, within a single context of learning. That is, we often draw conclusions about the relationship between the brain and human behavior based on either only the long-term learning (the relatively permanent changes in knowledge, i.e., competence) or the temporary fluctuation in behavior (momentary performance) (Heideman, van Ede, \& Nobre, 2018; Rose, Haider, Salari, \& Buchel, 2011; Thomas et al., 2004; Turk-Browne, Scholl, Johnson, \& Chun, 2010). On the other hand, if we measure the same cognitive functions at many different time points, we assume that only our manipulation (different study groups or conditions) determines the potential change in performance between the consecutive sessions (i.e., that the two performances are similar without manipulation). Many examples show that performance and competence can be separated from each other. They can differ due to fatigue, latent learning, 
practice, or overlearning of the practiced skill (Soderstrom \& Bjork, 2015). Thus, if we only measure performance, it could easily lead to wrong conclusions on brain-behavior relationships. In our third, complementary study, we tested how fragile are the memory representations that we are measuring by giving extreme instructions to the participants on an implicit probabilistic learning task. If instructions do not affect learning, it will underlie the robust nature of picking up statistical regularities (Kóbor et al., 2017); otherwise, it will raise our attention to the possible differences between competence and performance measured in learning and memory tasks. 


\section{AIMS AND OBJECTIVES}

The current thesis presents two TMS studies to address some of the issues mentioned above concerning the role of DLPFC in WM and implicit probabilistic learning (Table 1). Because of the inconsistent results of TBS methods to manipulate WM, Study I aimed to further investigate the effects of TBS on WM-related processes. The above-discussed gaps in the literature led us to the following questions: (1) does iTBS improve and cTBS worsen WM performance, (2) do left and right DLPFC stimulation lead to different changes in WM performance? We go beyond previous studies by directly comparing the effect of the two major types of TBS, and also by investigating the effect of TBS over the left and right DLPFC in the same experimental design. To answer our research questions, we administered iTBS, cTBS, or sham stimulation over the left and right DLPFC of healthy participants. Before and after the stimulation, the participants performed the n-back working memory task. We compared their behavioral performance over time, side of stimulation, and between the three experimental groups who received either iTBS, cTBS, or sham stimulation.

Next, Study II aimed to investigate the involvement of the same region, that is, the DLPFC, in implicit probabilistic learning. Our main question was whether DLPFC stimulation during learning affects the acquisition and retrieval of implicit probabilistic knowledge. We go beyond previous studies by multiple steps: by investigating the effects on probabilistic statistical regularities, by applying bilateral stimulation, and also by testing the aftereffects at several time points after the stimulation. Healthy participants performed the ASRT task, and in the rest periods, they either received $1 \mathrm{~Hz}$ inhibitory stimulation or sham stimulation bilaterally over the DLPFC. We compared their learning performance during learning, $10 \mathrm{~min}, 2 \mathrm{~h}$, and $24 \mathrm{~h}$ after the stimulation.

The last study investigated how a potential methodological problem can affect the behavioral results of the study of implicit probabilistic learning, i.e., how stable the statistical representations that we are measuring are. Thus, Study III aimed to test the effect of instruction on this type of learning. Our questions were 1) whether the speed/accuracy instructions affect the acquisition of probabilistic statistical regularities, and if they do, 2) do they affect the momentary performance and the retrieval of knowledge equally? To this aim, we trained participants on the ASRT task with speed or accuracy instructions, and after that, tested their knowledge by equally emphasizing speed and accuracy. We compared the learning (performance), as well as the retrieved knowledge (competence). 
Table 1. The main questions of the thesis and the methods used to answer them

The main question to be answered Methods

\begin{tabular}{|c|c|c|}
\hline Study I & $\begin{array}{l}\text { How does inhibitory and facilitatory TBS } \\
\text { over the left and right DLPFC affect WM } \\
\text { performance? }\end{array}$ & $\begin{array}{l}\text { Pre-and post-stimulation measurement on the n- } \\
\text { back WM task after one session of TBS over the } \\
\text { left and right DLPFC }\end{array}$ \\
\hline Study II & $\begin{array}{l}\text { How does inhibitory rTMS over bilateral } \\
\text { DLPFC affect implicit probabilistic } \\
\text { sequence learning performance? }\end{array}$ & $\begin{array}{l}\text { rTMS stimulation over bilateral DLPFC during } \\
\text { the acquisition of statistical regularities on the } \\
\text { ASRT task + post-stimulation retests }\end{array}$ \\
\hline Study III & $\begin{array}{l}\text { How do instructions affect learning and } \\
\text { retrieval of implicit probabilistic } \\
\text { knowledge? }\end{array}$ & $\begin{array}{l}\text { Acquisition of statistical regularities on the ASRT } \\
\text { task with speed/accuracy instructions + retest with } \\
\text { equally emphasizing speed and accuracy }\end{array}$ \\
\hline
\end{tabular}




\section{Study I - The effect of TBS on working memory}

\section{Participants}

Fifty-two healthy participants took part in the study. One participant was excluded due to technical failure in the first study session. Thus, final analyses were carried out on 51 participants ( 25 males, $\mathrm{M}_{\mathrm{age}}=23.68 \pm 3.06 \mathrm{SD}$ years). None of them had any neurological or psychiatric condition at the time of the study or report taking medications potentially influencing cognitive performance. All of them were TMS-naïve and signed informed consent before the start of the experiment. The study was conducted in accordance with the declaration of Helsinki and was approved by the local Ethics Committee (University of Szeged).

\section{Experimental design}

Participants took part in two separate sessions with at least two weeks of washout period between the two occasions (Fig. 1). They were randomly assigned to one of the three experimental groups: participants received cTBS $(n=17)$, iTBS $(n=18)$, or sham stimulation $(n=16)$. The left or the right DLPFC was stimulated in the first session and the other hemisphere in the second session, respectively; however, the type of stimulation (cTBS, iTBS, or sham) was kept constant between the two sessions. Before and after the stimulation, participants completed the three levels of the n-back working memory task (see section " $N$-back task") (Fig. 2).

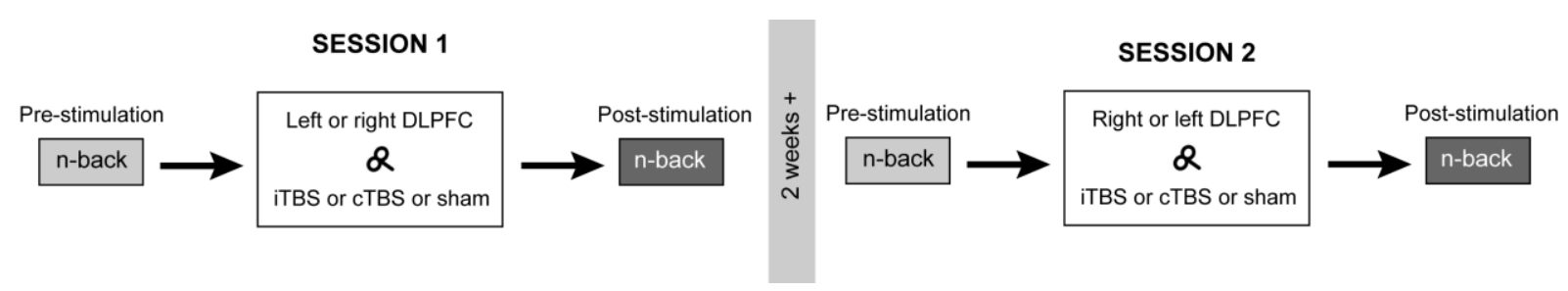

Fig. 1. Experimental design of Study I. The study consisted of two main sessions. In the first session, participants practiced three levels of the n-back WM task. After that, we administered iTBS, cTBS, or sham stimulation over the left or right DLPFC). After the stimulation, they completed the n-back task again. The second session occurred at least two weeks later; here, the experimental design was the same, except that the other hemisphere was stimulated. The type of stimulation (iTBS, cTBS, or sham) remained the same for the given participant. 


\section{Theta-burst stimulation and neuronavigation}

Participants of the cTBS and iTBS groups went through an anatomical T1-weighted MRI scan using a 1.5T GE Signa Excite HDxt scanner (Milwaukee, WI, USA): 3D IR-FSPGR: TR/TE/TI: 10.3/4.2/450 ms; fip angle: 15; ASSET: 2, FOC: $25 \times 25 \mathrm{~cm}$; matrix: 256×256; slice thickness: 1mm. We created 3D-brain models from the individual MRI scans and used a TMS Neuronavigator (Brain Innovation, Maastricht, the Netherlands) with ultrasound CMS20 Measuring System (Zebris GmbH, Tübingen, Germany) to localize the target position on the scalp of each participant.

We used the MagStim Rapid ${ }^{2}$ stimulator with a D70² $70 \mathrm{~mm}$ figure-of-eight coil (The Magstim Company Ltd, Whitland, Wales, UK) for the TBS. Right and left DLPFC were used as target areas and localized at the anterior third of the middle frontal gyrus (Brodmann 9/46). The coil was positioned over the target area, tangentially to the skull, with the handle pointing backward. For sham stimulation, the coil orientation was tilted by $45^{\circ}$ relative to the active stimulation conditions (only one wing of the coil was in contact with the scalp).

The cTBS pattern consisted of 3 pulses of $50 \mathrm{~Hz}$ (gamma frequency) in every $200 \mathrm{~ms}$ (theta-rhythm) for $40 \mathrm{~s}$ (600 pulses). The iTBS pattern consisted of $2 \mathrm{~s}-$ long trains repeated every $10 \mathrm{~s}$ for $190 \mathrm{~s}$ (600 pulses). The stimulation intensity was $30 \%$ of the maximal stimulator output.

To ensure that potential differences in the motor threshold (MT) did not influence the results, we measured the visible MT before the two TBS sessions (separate occasions). MT was defined as the lowest stimulation intensity applied over the right primary motor cortex required to evoke a visible contraction of the left abductor pollicis brevis muscle in 3 out of 5 probes. If $80 \%$ of the individual MT did not reach the used intensity, then the applied intensity was reduced by $20 \%$ of the MT value. This scenario occurred in one case in the cTBS group (intensity modified to $27 \%$ of maximum stimulator output, MSO) and in one case in the sham group (intensity modified to $29 \%$ of MSO). Overall, no difference was found between groups in terms of MT, $F_{(2,48)}=1.33, p=.28, \eta_{\mathrm{p}}^{2}=.52$.

\section{N-back task as a measure of working memory}

The n-back task was used to measure the WM performance of the participants (Gevins \& Cutillo, 1993) (Fig. 2). The 1-back, 2-back, and 3-back versions of the task were administered 
consecutively. PsychoPy was used as experimental software (version: v1.82.01) (Peirce, 2007). A capital letter stimulus was presented on the screen for $1500 \mathrm{~ms}$. Stimuli were chosen randomly out of 11 possible letters (A, C, E, I, K, L, S, O, R, T, U). Participants were instructed to press the space bar if the letter on the screen was identical to the letter presented one (1-back task), two (2-back task), or three (3-back task) trials earlier. After a 1500 ms-long interstimulus interval, the next item was presented. At each level, 100 trials were completed (a total of 300 trials per measurement). The frequency of target stimuli was set at $20 \%$. Reaction times (RT) and accuracy were recorded.

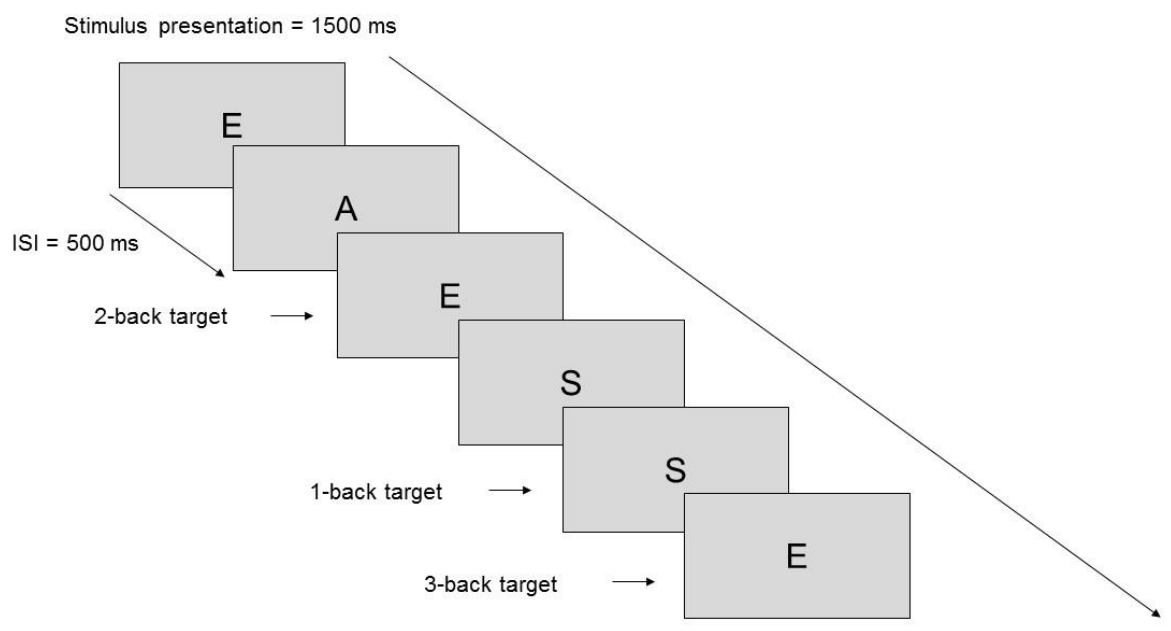

Fig. 2. The structure of the n-back WM task. The participants' task was to press a button if they perceived the same letter as presented one (1-back), two (2-back), or three (3-back) trials earlier. Each stimulus was presented on the screen for $1500 \mathrm{~ms}$, with an interstimulus interval of $500 \mathrm{~ms}$. Figure 2 of Vékony et al. (2018), see Appendix $I$.

\section{Statistical analysis}

The n-back performance was evaluated by the median RTs and discriminability index (d') (Haatveit et al., 2010). For the latter, four types of answers were distinguished: hits (correctly identified targets), misses (incorrectly identified targets as non-targets), false alarms (incorrectly identified non-targets as targets), and correct rejections (correctly identified nontargets). The $d^{\prime}$ scores were calculated as follows:

$$
d^{\prime}=Z(\text { hit rate })-Z(\text { false alarm rate })
$$

Mixed-design ANOVAs were conducted separately for RTs and $d^{\prime}$ scores. For the RT analysis, the within-subject factors of cognitive load (Load: 1-back vs. 2-back vs. 3-back), time 
of administration (Time: pre-stimulation vs. post-stimulation) and side of the stimulation (Side: right DLPFC vs. left DLPFC) and the between-subjects factor of stimulation type (Group: cTBS vs. iTBS vs. sham stimulation) were used.

Participants completed the 1-back task with high accuracy (99.53\% in total). Thus, we did not calculate $d^{\prime}$ scores for this sub-task. D' scores were analyzed with a similar mixed-design ANOVA but excluding the 1-back condition. An additional mixed-design ANOVA with the within-subject factor of Load and Side, and with the between-subjects factor of Group was performed to test whether the groups differed in their pre-stimulation n-back performance. Partial eta-squared $\left(\eta_{p}^{2}\right)$ is reported as the measure of effect size (considered low between .01 and .05, medium between .06 and .13, and high above .14) (Cohen, 1992).

\section{Study II - The effect of rTMS on probabilistic learning}

\section{Participants}

Thirty-two participants were recruited to participate in the study. All participants were right-handed. One participant was excluded from the final sample due to poor performance on the Berg Card Sorting Task (percentage of preservative errors: 31.25\%; percentage of correct responses: $51.56 \%$ ). Thus, the final analyses were carried out on 31 participants (four males, $\mathrm{M}_{\mathrm{age}}=22.16 \pm 3.01 \mathrm{SD}$ years). None of the participants had neurological or psychiatric disorders at the time of the experiment or report taking medication potentially affecting cognitive performance. The participants were TMS-naïve. The experiment was conducted in accordance with the Declaration of Helsinki and with the approval of the local Ethics Committee (University of Jena).

\section{Experimental design}

The experiment consisted of four sessions. In the first session, participants practiced on the Alternating Serial Reaction Time Task (ASRT) for ca. 25 minutes (Training/rTMS phase). Before starting the task, participants received rTMS bilaterally (5 minutes over the left and 5 minutes over the right hemisphere). Two groups were formed: one group of participants received $1 \mathrm{~Hz}$ rTMS over the DLPFC (Brodmann 9), and the other group received sham stimulation. After every ca. five minutes of learning, the rTMS procedure was repeated (repeated four more times, i.e., five runs of stimulation altogether). The second, third, and fourth session took place $10 \mathrm{~min}, 2 \mathrm{~h}$, and $24 \mathrm{~h}$ after the end of the Training/rTMS phase, respectively. 
Participants completed ca. 5 more minutes of practice on the ASRT task in every session (Fig. $3)$.

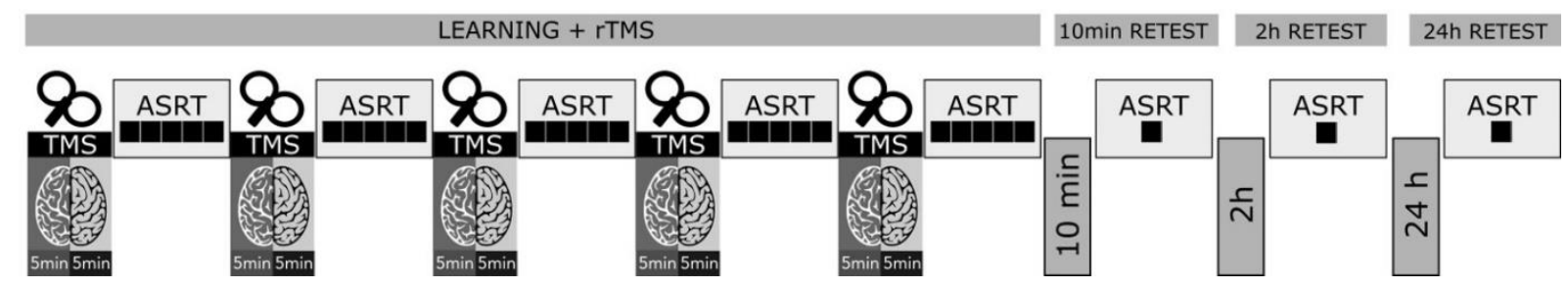

Fig. 3. Experimental design of Study II. The experiment consisted of four sessions. In the first session, participants received $1 \mathrm{~Hz}$ rTMS or sham stimulation bilaterally over the DLPFC for 5-5 minutes. After that, they practice on the ASRT task for ca. 5 minutes (completion of 5 blocks). This procedure was repeated four more times $(5 \times 5-5$ minutes and $5 \times 5$ blocks altogether). After the last stimulation, a $10 \mathrm{~min}$ break followed, and then five more blocks of ASRT were administered. We also measured the performance on the ASRT task $2 \mathrm{~h}$ and $24 \mathrm{~h}$ after the end of the rTMS. Modified version of Figure 1 of Ambrus et al. (2020), see Appendix II.

After the end of the ASRT task of the last session, participants completed the Berg Card Sorting Task (Fox, Mueller, Gray, Raber, \& Piper, 2013) and the Counting Span task (Case, Kurland, \& Goldberg, 1982; Conway et al., 2005; Engle, Laughlin, Tuholski, \& Conway, 1999) to ensure that the participants' executive function performance is equal in the two groups. We observed no significant differences in executive functions between the two groups (Berg Card Sorting Test, percent correct responses: $p=.80$; percent perseverative errors: $p=.38$; percent non-perseverative errors: $p=.65$; Counting span: $p=.23$ ). Participants also filled out a questionnaire about the perceived task difficulty, discomfort, and tiredness. Again, no differences were found either in the level of discomfort $(p=.57)$, tiredness $(p=.83)$, or perceived task difficulty $(p=.24)$.

\section{rTMS and neuronavigation}

Before the TMS sessions, the individual structural MRIs of the participants were collected using a Siemens Magnetom Trio 3 T MRI scanner at the Institute for Diagnostic and Interventional Radiology, University of Jena. We acquired high-resolution sagittal T1-weighted images for the 3D head and brain meshes using a magnetization EPI sequence (MP-RAGE; TR $=2300 \mathrm{~ms} ; \mathrm{TE}=3.03 \mathrm{~ms} ; 1 \mathrm{~mm}$ isotropic voxel size). The 3D-head and brain models were created from the participants' individual MRI scans.

A PowerMag 100 Research Stimulator (MES Forschungssysteme GmbH) was used for the delivery of rTMS, and a PowerMag View (MES Medizintechnik GmbH) Neuronavigation system was used to carry out neuronavigation. The target area was the dorsal part of the DLPFC (MNI coordinates: $\mathrm{x}=37, \mathrm{y}=33, \mathrm{Z}=32$ ). For sham stimulation, the coil was oriented 
perpendicularly, facing away from the skull. Magnetic pulses of $1 \mathrm{~Hz}$ were delivered at $55 \%$ MSO. In one run, 300 pulses were delivered (one run per hemisphere, repeated five times). The stimulation started with the right or the left hemisphere (counterbalanced between participants) but the order remained the same for the given participant.

\section{ASRT as a measure of implicit probabilistic learning}

Implicit probabilistic learning was measured with the ASRT task (Howard et al., 2004; Song et al., 2007). Four circles were presented on a computer screen in a horizontal arrangement. A target stimulus (a drawing of a dogs' head) appeared in one of the four possible locations (Fig. 4A). The participants were instructed to press the response key corresponding to the position of the target stimulus ( $\mathrm{Z}, \mathrm{C}, \mathrm{B}$, and $\mathrm{M}$ on a QWERTY keyboard) as fast and as accurately as possible. The key "Z" and " $\mathrm{C}$ " had to be pressed with the middle and index finger of the left hand, and the "B" and "M" with the middle and index finger of the right hand. The stimulus remained on the screen until the first correct response. After a $120 \mathrm{~ms}$ long responsethe-stimulus interval, the next stimulus appeared. Eighty-five stimuli were presented in a block. After each block, participants received feedback about their average speed and accuracy during the block and could rest a little. In the first session (Training/rTMS phase), 25 blocks were completed. Before every unit of five blocks (one epoch), the 5-5 min long rTMS runs were administered. Five blocks were completed in each of the $10 \mathrm{~min}, 2 \mathrm{~h}$, and 24 h retest phases.

The first five trials of each block were presented randomly and served practice purposes. After that, an eight-element alternating sequence of pattern and random elements was repeated ten times. For example, the sequence could have been $2 \mathrm{r} 3 \mathrm{r} 4 \mathrm{r} 1 \mathrm{r}$, where the numbers indicate the location of the target stimulus from left to right, and " $r$ " indicates a random position. Due to this structure, some combinations of three consecutive elements (referred to as triplets) appeared with a higher probability than other triplets. For instance, if the sequence was $2 \mathrm{r} 4 \mathrm{r} 3 \mathrm{r} 1 \mathrm{r}$, the $2 \mathrm{X} 4,4 \mathrm{X} 3,3 \mathrm{X} 1$, and $1 \mathrm{X} 2$ triplets (where "X" indicates any middle element) occurred with higher probability because the third element of the triplet could be derived from the pattern elements (and in some cases, from random elements too, but with less probability). The triplets of, for example, $2 \mathrm{X} 1$ or $3 \mathrm{X} 2$ could appear with less probability because the third element could only be random (Fig. 4B). The third element of a high-probability triplet is more predictable than the third element of a low-probability triplet; therefore, as time progressed, participants become faster and faster in responding to the third element of high-probability triplets. The ratio of high-probability triplets was $62.5 \%$ (16 different triplets, each of them occurs in $4 \%$ of the 
cases), and the ratio of low-probability triplets was $37.5 \%$ (48 different triplets, each of them occurs in $\sim 0.8 \%$ of the cases) (Fig. $4 \mathrm{C}$ ).

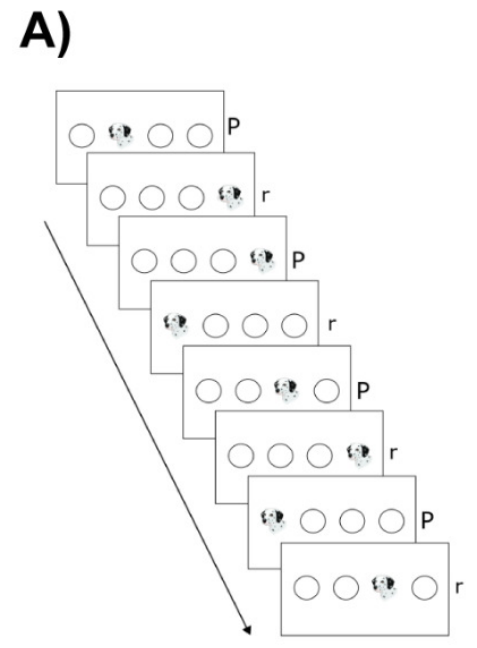

B) Sequence: $2 \mathrm{r} 4 \mathrm{r} 3 \mathrm{r} 1 \mathrm{r}$

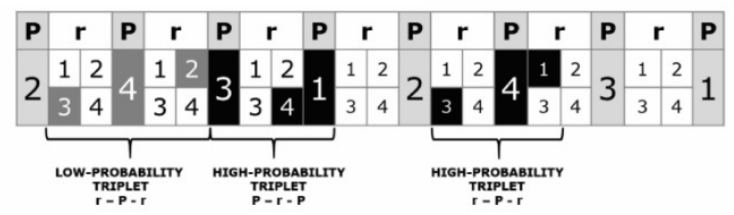

C)

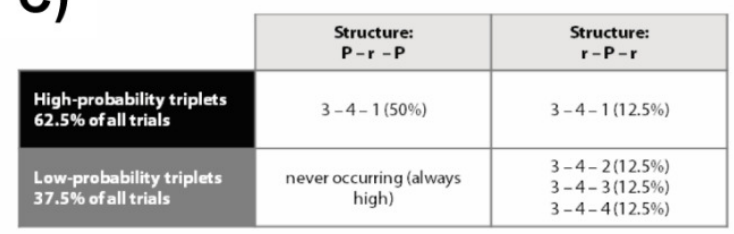

Fig. 4. Structure of the ASRT task. (A) In the ASRT task, a stimulus (a head of a dog) appears at one of four possible positions. (B) The order of appearance follows a predetermined, 8-element sequence: every first element of this sequence is part of the pattern, and every second element appears at a truly random position. Because of this hidden sequence, specific triplets (three consecutive elements) appear with higher probability than other triplets. (C) High-probability triplets can be formed by two pattern elements and one random element in the middle. Two random elements and a pattern element in the middle can also form high-probability triplets; however, much less frequently. Low-probability triplets always consist of two random and one pattern element. Modified version of Figure 1 of Vékony et al. (2020), see Appendix III.

\section{Statistical analysis}

We categorized every stimulus as the third element of a high- or low-probability triplet. Trills (e.g., 1-3-1) and repetitions (e.g., 3-3-3) were excluded from the analysis, as participants tend to show pre-existing response tendencies to these types of triplets (Howard \& Howard, 1997; Howard et al., 2004). Only correct responses were considered. Trials with \pm 2.5 SD above or below the mean of the given epoch (units of analysis, five blocks) were also removed. We calculated the individual mean of RTs separately for high- and low-probability triplets in each epoch. After that, we subtracted the RTs for high-probability triplets from the RTs for the lowprobability triplets and divided it by the RTs for low-probability triplets. This learning index was used to compare the learning of the two groups in the four experimental sessions (for the Training/rTMS phase, the learning indices for the five epochs were averaged).

We performed a mixed-design ANOVA with the between-subjects factor of Group (DLPFC Stimulation vs. Sham Stimulation) and the within-subject factor of Session (Training/rTMS session vs. 10 min retest session vs. $2 \mathrm{~h}$ retest session vs. $24 \mathrm{~h}$ retest session) 
to compare the statistical learning performance between the two stimulation groups throughout the experiment. We also tested whether the observed effects were due to the order of stimulation. Thus, we conducted a mixed-design ANOVA with the between-subjects factor of Order (Right Start vs. Left Start) and the within-subject factor of Session (Training/rTMS session vs. 10 min retest session vs. 2 h retest session vs. 24 h retest session). GreenhouseGeisser corrections were used if applicable. Partial eta-squared $\left(\eta_{\mathrm{p}}^{2}\right)$ is reported as the measure of effect size. Bonferroni-corrected pairwise comparisons were used to further analyze significant main effects and interactions. Alpha level was set at .05

\section{Study III - The effect of instructions on probabilistic learning}

\section{Participants}

Sixty-six healthy young adults were recruited for the study. Five of them were excluded from the experiment due to misunderstanding of task instructions (RT or accuracy scores more than 2 SD from the mean of their group in more than $50 \%$ of the epochs, i.e., units of analysis). Therefore, the final analysis was carried out on 61 participants ( 40 females, $M_{\text {age }}=21.18 \pm 2.13$ SD years). None of them suffered from any neurological and/or psychiatric disorders or reported taking medications potentially influencing cognitive performance. All participants gave written informed consent before enrollment and received course credit in exchange for participation. The study was approved by the local Ethics Committee (Eötvös Loránd University), and it was conducted in accordance with the Declaration of Helsinki.

\section{Experimental design}

Participants completed three practice blocks of 85 random trials of the ASRT task. It was followed by the training session (referred to as Different Instruction Phase). Participants completed four epochs (20 blocks) of the ASRT task (the ASRT task was used with the same parameters as in Study II, Fig. 4). However, we gave different instructions to the two groups. The Accuracy Group ( $n=31$ ) were instructed to be as accurate as possible during the task. The Speed Group $(n=30)$ were instructed to be as fast as possible. After a $\sim 10$ min rest period, the next session followed. In the Same Instruction Phase, participants completed one more epoch of ASRT (five blocks). Here, both groups were instructed to complete the task as quickly and as accurately as possible (Fig. 5). After the ASRT task, a process dissociation procedure was administered to measure the level of implicitness (Jacoby, 1991), and also a questionnaire 
dealing with the subjective preferences of the participants about speed and accuracy (see details in Appendix III).
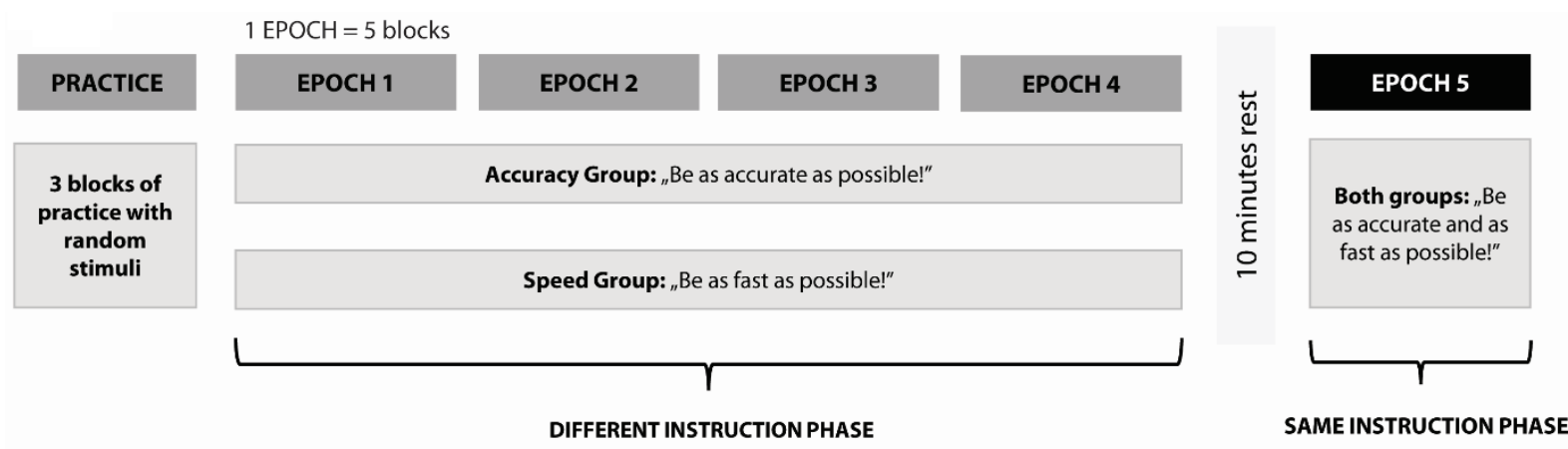

Fig. 5. Experimental design of Study III. First, three blocks of practice with random stimuli were completed. After that, four epochs of the ASRT task were administered. The Accuracy Group received the instruction to be as accurate as possible, and the Speed Group to be as fast as possible (Different Instruction Phase). After that, 10 min rest period followed. After the rest period, participants completed another epoch of the ASRT task, but this time, both groups were instructed to be fast and accurate at the same time. Modified version of Figure 1 of Vékony et al. (2020), see Appendix III.

\section{Statistical analysis}

Each trial was categorized as the third element of a high- or low-probability triplet. Trills and repetitions were excluded (J. H. Howard \& Howard, 1997; D. V. Howard et al., 2004). Only correct responses were considered. The individual median RTs and accuracies were calculated separately for high- and low-probability triplets in each epoch.

The learning performance between the two groups in the Different and Same Instruction Phase was compared with mixed-design ANOVAs. We performed the ANOVAs with the within-subject factor of Triplet (high- vs. low-probability triplets) and the between-subjects factor of Group (Accuracy Group vs. Speed Group). For the analysis of the Different Instruction Phase, the Epoch factor (Epoch 1-4) was also included.

Greenhouse-Geisser epsilon $(\varepsilon)$ correction was used if necessary. We reported corrected $d f$ and $p$ values if applicable. Partial eta-squared $\left(\eta_{\mathrm{p}}^{2}\right)$ is reported as the measure of effect size. We used the Least Significant Difference tests for pairwise comparisons, and significant interactions involving the Triplet factor were further analyzed using follow-up ANOVAs on the difference scores between high- and low-probability triplets. Alpha level was set at .05. Bayesian analyses were also conducted to support further our findings (see details and results in Appendix III). 


\section{RESULTS}

\section{Study I - The effect of TBS on working memory}

\section{Did TBS affect RTs in the n-back task?}

The ANOVA of the RTs revealed a significant main effect of Time, $F_{(1,48)}=8.76, p=$ $.005, \eta_{\mathrm{p}}^{2}=.15$. Pairwise comparisons confirmed shorter overall median RTs following stimulation $\left(\mathrm{M}_{\text {pre-stimulation }}=618 \mathrm{~ms} \pm 12 \mathrm{~ms} \mathrm{SE}, \mathrm{M}_{\text {post-stimulation }} 600 \mathrm{~ms} \pm 11 \mathrm{~ms} \mathrm{SE}\right)$. The main effect of Load was also significant, $F_{(2,96)}=115.23, p<.001, \eta_{\mathrm{p}}{ }^{2}=.71$. RTs increased with higher cognitive load (M1-back: $499 \mathrm{~ms} \pm 9 \mathrm{~ms} \mathrm{SE}, \mathrm{M}_{2 \text {-back: }} 609 \mathrm{~ms} \pm 14 \mathrm{~ms} \mathrm{SE} ; \mathrm{M}_{2 \text {-back: }} 719 \mathrm{~ms}$ \pm 0.017 SE. The interaction of Time and Load factors was also significant, $F_{(1,96)}=4.95, p=$ $.009, \eta_{\mathrm{p}}{ }^{2}=.09$. A change in RTs between the pre- and post-stimulation measurements was found for the 2-back ( $p=.004)$ and 3-back conditions $(p=.01)$ but not for 1-back condition $(p=0.45)$. The above interaction was not modified by the Group, $F_{(4,96)}=1.36, p=.25, \eta_{\mathrm{p}}{ }^{2}=.05$, or by the Side factors, $F_{(1.69,81.10)}=0.31, p=.69, \eta_{\mathrm{p}}^{2}=.01$. The lack of the role of the Group factor was also confimed by Bayesian analysis methods (see details in Appendix I.).

\section{Did TBS affect discriminability in the n-back task?}

The ANOVA of the $d$ ' scores revealed a significant main effect of Time, $F_{(1,48)}=30.30$, $p<0.001, \eta_{\mathrm{p}}{ }^{2}=0.39$. Increased $d$ ' scores were found following the stimulation $\left(\mathrm{M}_{\text {pre-stimulation: }}\right.$ $2.71 \pm 0.07 \mathrm{SE}, \mathrm{M}_{\text {post-stimulation: }} 2.99 \pm 0.07 \mathrm{SE}$ ). The main effect of Load was also significant, $F_{(1,48)}=344.82, p<.001, \eta_{\mathrm{p}}{ }^{2}=.88$. Participants performed better in the 2-back condition than in the 3-back condition ( $\mathrm{M}_{2 \text {-back: }} 3.58 \pm 0.07 \mathrm{SE}$; M3-back: $\left.2.13 \pm 0.09 \mathrm{SE}\right)$. Most importantly, a significant interaction between the Time and Group was detected, $F_{(2,48)}=4.25, p=.02, \eta_{\mathrm{p}}{ }^{2}=$ .15. Pairwise comparisons revealed an increase of $d$ 'scores from pre- to post-stimulation measurement in the iTBS $(p=.001)$ and the sham group $(p<0.001)$. However, no improvement was found in the cTBS group $(p=.32)$ (Fig. 6). The Time $\times$ Group interaction was not modified by the Side factor, $F_{(1,48)}=0.20, p=.82, \eta_{\mathrm{p}}^{2}=.008$ (also confirmed by Bayesian methods, see Appendix I). 
A) LEFT DLPFC

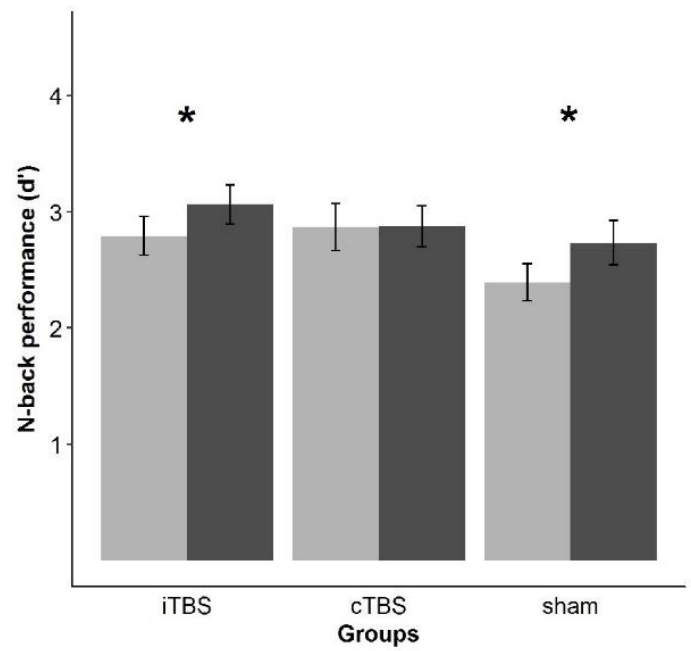

B) RIGHT DLPFC

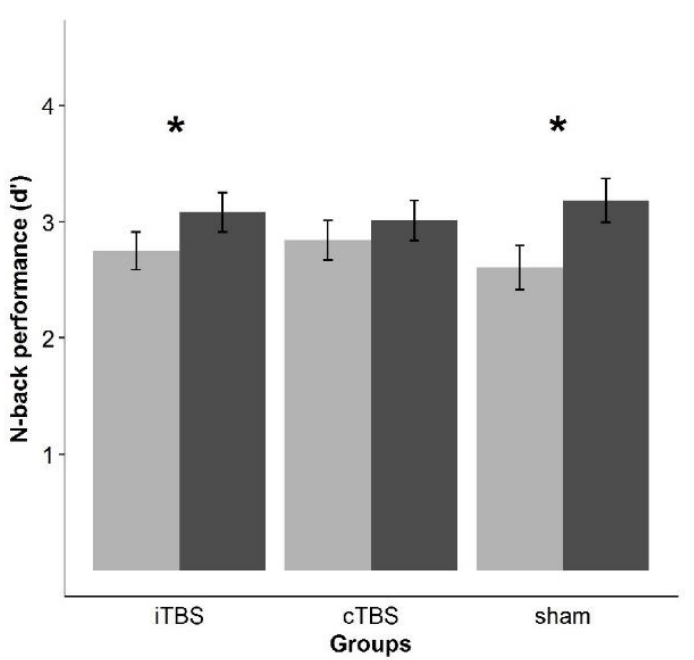

Fig. 6. $N$-back performance of the three groups before and after the stimulation of (A) the left and (B) right DLPFC. The horizontal axes denote the three groups, and the vertical axis the $d$ '. The error bars indicate SEM. The results were the same after the stimulation of both hemispheres: after iTBS and sham stimulation, practice effect occurred, i.e., the performance enhanced compared to the pre-stimulation measurement. However, after the cTBS, a lack of change was observed. $* p<.05$ Figure 1 of Vékony et al. (2018), see Appendix I.

\section{Study II - The effect of rTMS on probabilistic learning}

The ANOVA revealed a significant main effect of Session, $F_{(3,87)}=7.11, p<.001, \eta_{\mathrm{p}}{ }^{2}$ $=.20$. The pairwise comparisons demonstrated an overall increase in performance in the three retest sessions compared to the Training/rTMS session (Training/rTMS session vs. $10 \mathrm{~min}$ retest session: $p=.001,2 \mathrm{~h}$ retest session: $p=.001,24 \mathrm{~h}$ retest session: $p=.004)$. No main effect of Group was found, $F_{(1,29)}=0.61, p=.44, \eta_{\mathrm{p}}{ }^{2}=.02$. Importantly, the interaction between the Session and Group factors was significant, $F_{(3,87)}=3.96, p=.01, \eta_{\mathrm{p}}{ }^{2}=.12$. An increased performance was detected in the 10 min retest session in the Sham Group compared to the Training/rTMS session ( $p=.002$ ). Performance of DLPFC Group was also enhanced at the $2 \mathrm{~h}$ retest session compared to the Training/rTMS session $(p=.01)$. Contrary to the Sham Group, the difference was still statistically significant at the $24 \mathrm{~h}$ retest session $(p=.001)$. The learning indices between the two groups was similar at all measurements (each $p>.25$ ) except for the $24 \mathrm{~h}$ retest session, where the DLPFC Group showed better probabilistic knowledge $(p=.03)$ (Fig. 7). 

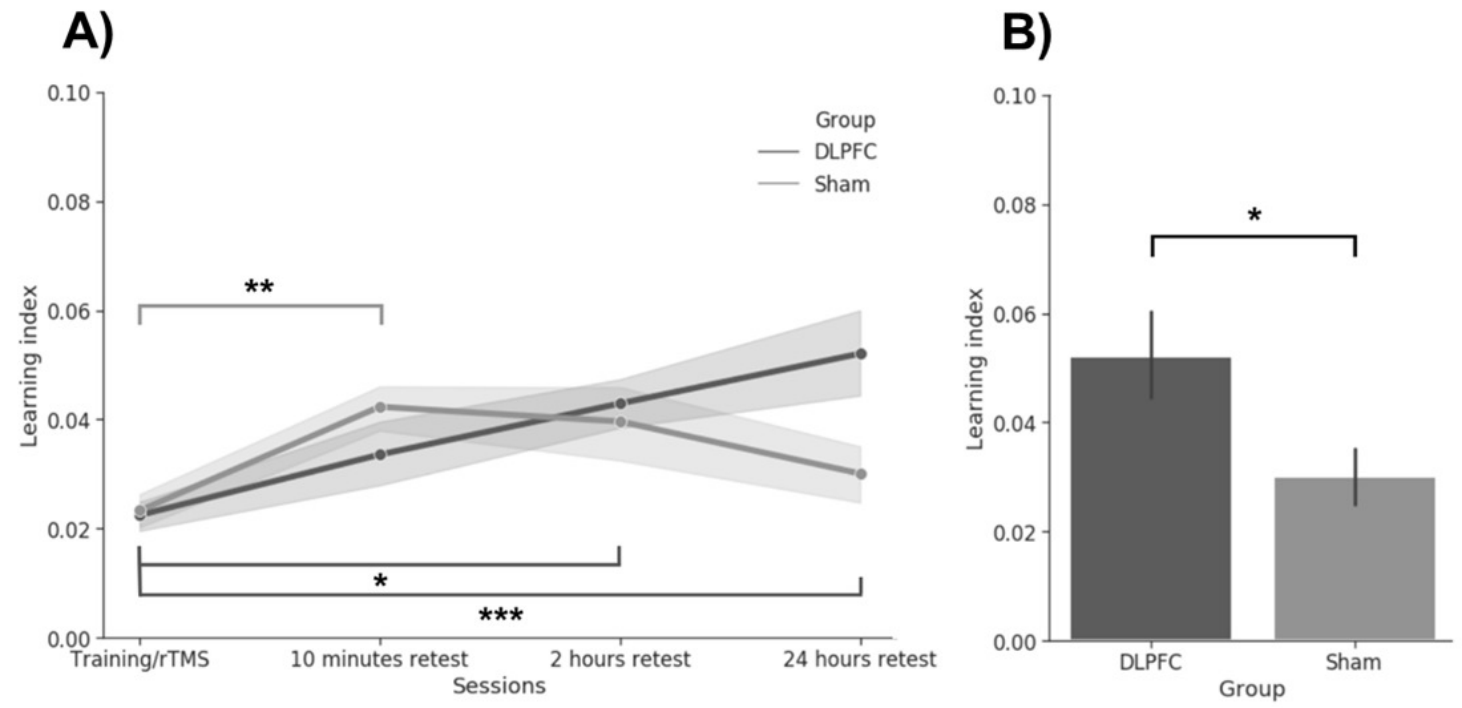

Fig. 7. Learning performance of the two groups on the ASRT task. (A) Changes in performance over the four sessions. The horizontal axis shows the four measurement points, and the vertical axis the learning indices (calculated as [mean RTs for low-probability triplets - mean RTs for high-probability triplets]/mean RTs for lowprobability triplets). Thus, higher scores indicate better learning of the sequence. In the Sham Group, performance improved from the Training/rTMS session to the 10 min retest session. In the DLPFC Group, on the other hand, performance enhanced between the Training/rTMS session and the 2-hour retest session, and also between the Training/rTMS session and the 24-hour retest session. (B) Performance of the two groups on the 24-hour retest session. DLPFC Group showed significantly higher probabilistic knowledge than the Sham Group. * $p<.05$ Modified version of Figure 2 of Ambrus et al. (2020), see Appendix II.

The ANOVA regarding the stimulation order of the hemispheres did not reveal any difference in probabilistic learning performance, $F_{(3,42)}=0.15, p=.93, \eta_{\mathrm{p}}^{2}=.01$.

\section{Study III - The effect of instructions on probabilistic learning}

\section{Did the instructions affect general RTs and probabilistic learning in $R T$ measures in the}

\section{Different Instruction Phase?}

The ANOVA on the RT scores in the Different Instruction Phase revealed a main effect of Group, $F_{(1,59)}=51.86, p<.001, \eta_{\mathrm{p}}^{2}=.47$, indicating faster overall RTs in the Speed Group. This result means that the instructions modified the overall speed of the participants. A main effect of Epoch was revealed, $F_{(1.97,116.33)}=7.46, p=.001, \eta_{\mathrm{p}}{ }^{2}=.11$, indicating a change in average RTs during the task. The interaction between the Epoch and Group factors was nonsignificant, $F_{(1.97,116.33)}=2.30, p=.10, \eta_{\mathrm{p}}^{2}=.04$.

The ANOVA revealed a main effect of Triplet, $F_{(1,59)}=49.41, p<.001, \eta_{\mathrm{p}}{ }^{2}=.46$, as participants were faster for high- compared to low-probability triplets, thus, revealing significant implicit probabilistic learning. Importantly, no significant interaction was found between the Triplet and Group factors, $F_{(1,59)}=0.48, p=.49, \eta_{\mathrm{p}}{ }^{2}=.01$. These results indicate 
that the degree of learning did not differ between groups throughout the learning. The interaction of the Triplet and Epoch factors was significant, $F_{(3,177)}=5.66, p=.001, \eta_{\mathrm{p}}^{2}=.09$, indicating that the degree of learning changes throughout the task. However, the interaction between the Triplet, Epoch, and Group factors was non-significant, $F_{(3,177)}=0.90, p=.43, \eta_{\mathrm{p}}^{2}$ $=.02$. These results indicate that the time course of probabilistic learning did not differ between groups (Fig. 8). The analysis with the standardized learning scores in the RT measures revealed similar results, and Bayesian analysis confirmed the lack of difference between groups in terms of implicit probabilistic learning (see Appendix III for details).

A) Accuracy Group

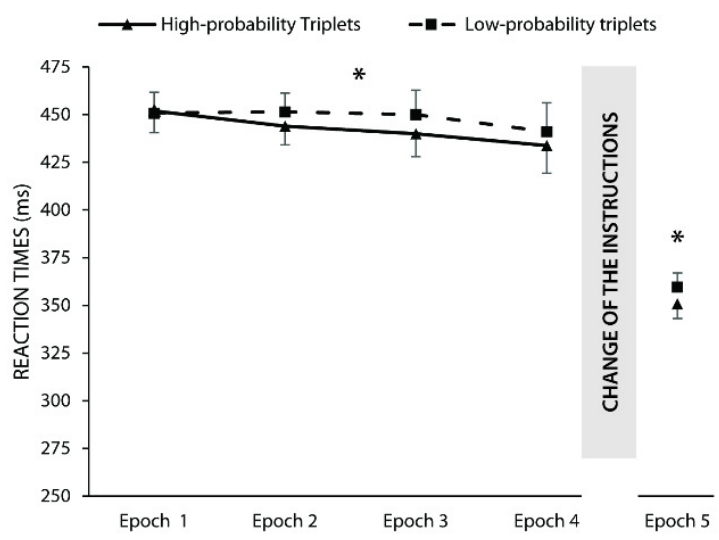

B) Speed Group

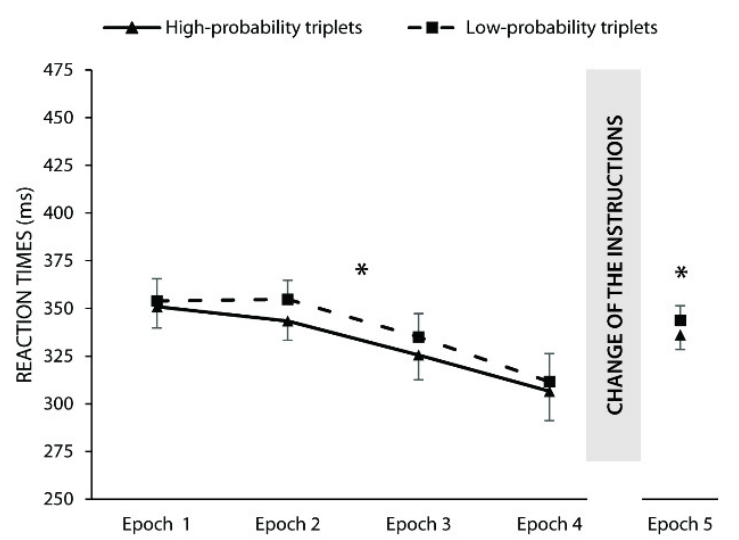

Fig. 8. Performance of the (A) Accuracy Group and (B) Speed Group in RT measures. The horizontal axes indicate the five epochs of the task (Epoch 1 - 4 as part of the Different Instruction Phase, and Epoch 5 as part of the Same Instruction Phase). The vertical axes denote RTs. The solid lines represent RTs for high-probability triplets, and the dashed lines the same for low-probability triplets. Similar levels of implicit probabilistic knowledge were detected in both groups, and it remained even after the change of the instructions. $* p<.05$. Figure 3 of Vékony et al. (2020), see Appendix III.

\section{Did the instructions affect general accuracies and probabilistic learning in accuracy measures in the Different Instruction Phase?}

The ANOVA on the accuracy scores in the Different Instruction Phase revealed a significant main effect of Group, $F_{(1,59)}=117.40, p<.001, \eta_{\mathrm{p}}{ }^{2}=.67$, indicating higher overall accuracy in the Accuracy Group. This result means that the instructions influenced the overall accuracy of the participants. The main effect of Epoch was also significant, $F_{(1.81,107)}=8.19, p$ $=.001, \eta_{\mathrm{p}}{ }^{2}=.13$, revealing a significant decrease in accuracies as the task progressed. The interaction between the Epoch and Group factors was also significant, $\mathrm{F}_{(1.84,107)}=7.08, p=$ $.002, \eta_{\mathrm{p}}{ }^{2}=.11$. This result indicates that accuracy decreased over the epochs in the Speed Group but not in the Accuracy Group. 
The ANOVA revealed a main effect of Triplet, $F_{(1,59)}=93.88, p<.001, \eta_{\mathrm{p}}{ }^{2}=.61$. Participants were more accurate on high- compared to low-probability triplets, revealing implicit probabilistic learning. Here, the ANOVA revealed a significant interaction between the Triplet and Group factors, $F_{(1,59)}=45.25, p<.001, \eta_{\mathrm{p}}^{2}=.43$. Only the Speed Group showed implicit statistical learning, i.e., more accurate responses to high- compared to the lowprobability triplets. The interaction between the Triplet and Epoch factors was significant, $F_{(3 \text {, }}$ 177) $=3.69, p=.01, \eta_{\mathrm{p}}{ }^{2}=.06$. It indicates that the degree of statistical learning changed throughout learning (Fig. 9). The three-way interaction of the Triplet, Epoch, and Group factors was also significant, $F_{(2.95,174.28)}=2.99, p=.03, \eta_{\mathrm{p}}{ }^{2}=.05$, suggesting different dynamics of implicit probabilistic learning for the two groups: only the Speed Group showed increasing probabilistic learning with time. The analysis of the standardized learning scores revealed similar results, and Bayesian analysis confirmed again the lack of difference between groups in terms of implicit probabilistic learning (see Appendix III for details).

A) Accuracy Group

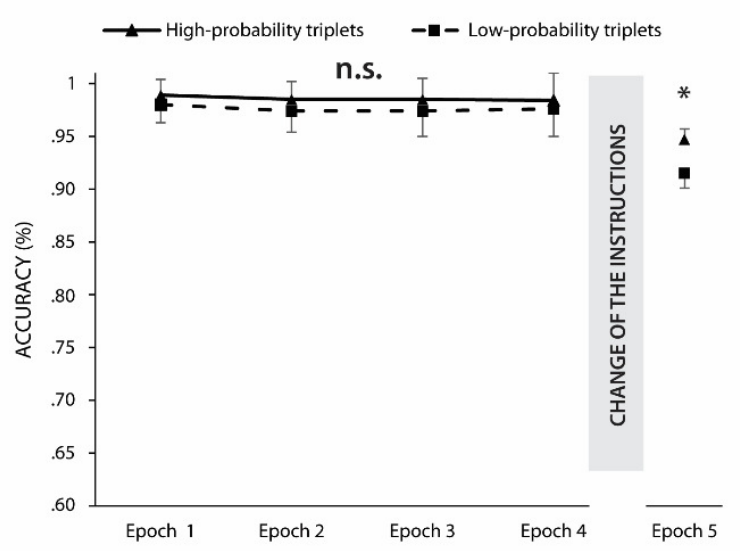

B) Speed Group

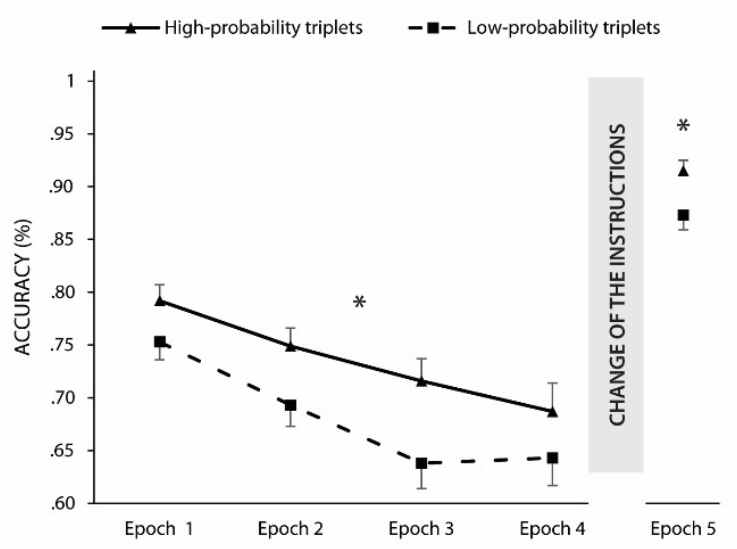

Fig. 9. Performance of the (A) Accuracy Group and (B) Speed Group in accuracy measures. The horizontal axes indicate the five epochs of the task (Epoch 1 - 4 as part of the Different Instruction Phase, and Epoch 5 as part of the Same Instruction Phase). The vertical axes denote accuracies. The solid lined represent accuracies for high-probability triplets, and the dashed lines the same for low-probability triplets. Implicit probabilistic learning was detected only in the Speed Group during the Different Instruction Phase, but a similar level of knowledge was found in both groups in the Same Instruction Phase. *: $p<.05$, ns: $p>.05$. Figure 4 of Vékony et al. (2020), see Appendix III.

\section{Did the acquired knowledge differ between groups in the Same Instruction Phase?}

The ANOVA on the RT measures of the Same Instruction Phase revealed a main effect of Triplet, $F_{(1,59)}=50.50, p<.001, \eta_{\mathrm{p}}{ }^{2}=.46$. Again, participants were faster for high-probability triplets, indicating that probabilistic knowledge persisted in this phase. No main effect of Group was found, $F_{(1,59)}=2.03, p=.16, \eta_{\mathrm{p}}{ }^{2}=.03$, indicating that after the change of the instructions, the difference in overall RTs between groups disappeared. Most importantly, the interaction of 
the Triplet and Group factors was non-significant, $F_{(1,59)}=0.27, p=.60, \eta_{\mathrm{p}}{ }^{2}=.01$. This result indicates that the two groups showed the same level of statistical knowledge in the Same Instruction Phase (Fig. 10).

\section{Reaction Times Measures}

A)

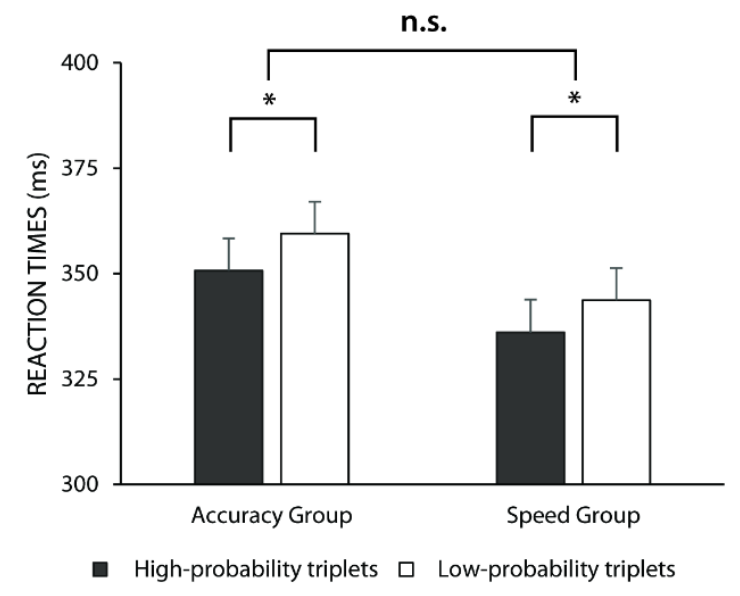

C)

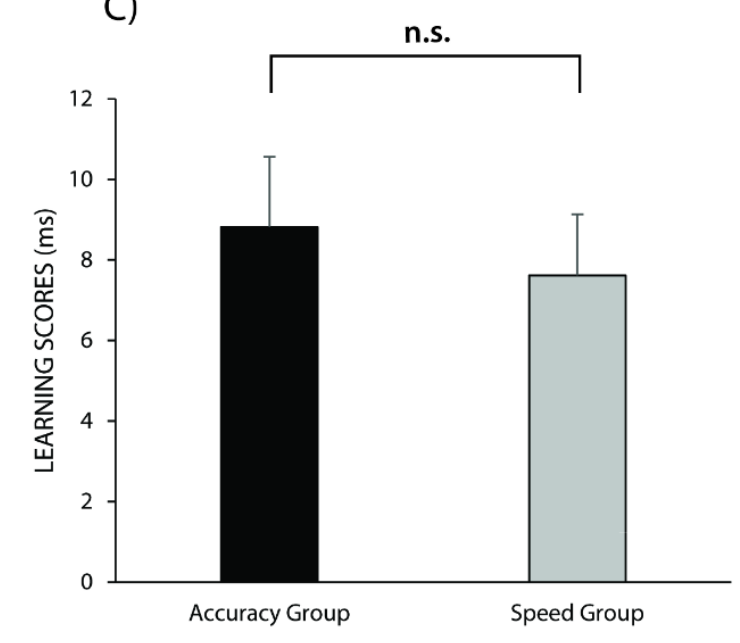

Accuracy Measures

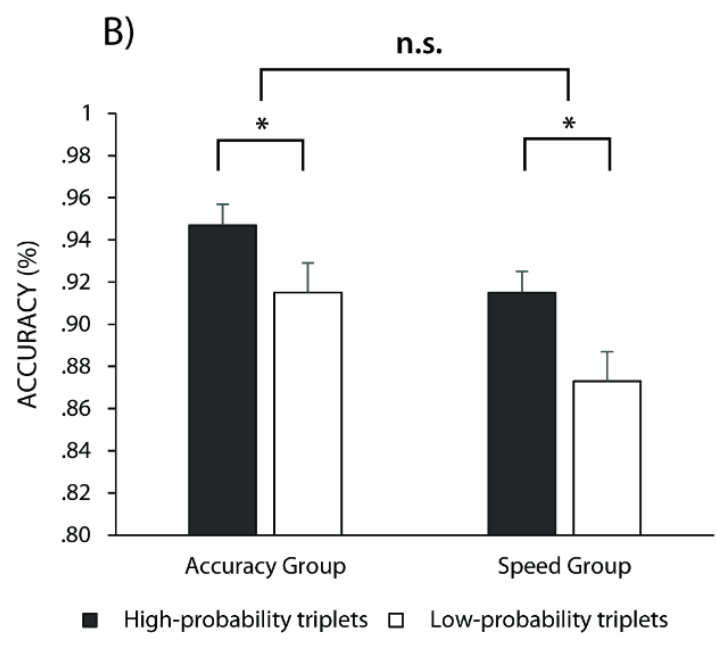

D)

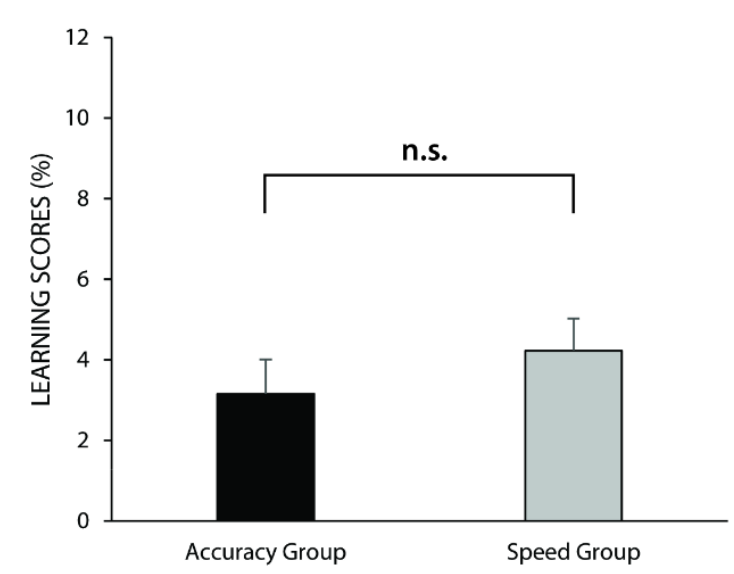

Fig. 10. Comparison of the high-and low-probability triplets ( $A$ and $B$ ), and the learning scores in the Same Instruction Phase (C and D). The vertical axes denote the RTs (A), accuracy (B), or the learning scores (C and D). The horizontal axes indicate the two groups. The error bars denote the SEM. Although probabilistic knowledge was detected in both groups, a lack of significant difference was found in the learning scores. *: $p\langle .05$, ns: $p>$ .05. Figure 5 of Vékony et al. (2020), see Appendix III.

The ANOVA on the accuracy scores revealed a significant main effect of Triplet, $F_{(1,59)}$ $=39.96, p<.001, \eta_{\mathrm{p}}{ }^{2}=.40$, indicating persisting statistical knowledge in accuracy: more accurate responses were found for high- compared to low-probability triplets. The ANOVA revealed a significant main effect of Group, $F_{(1,59)}=5.08, p=.03, \eta_{\mathrm{p}}{ }^{2}=.08$. It indicates that the overall difference in accuracy remained after the change of the instructions. Most importantly, the interaction between the Triplet and Group factors was non-significant, $\mathrm{F}_{(1,59)}=0.85, p=$ 
$.36, \eta_{\mathrm{p}}{ }^{2}=.01$. This result indicates a similar level of probabilistic knowledge in the two groups after the change of the instructions (Fig. 10).

The analysis with the standardized learning scores in RT and accuracy measures revealed similar results, and the Bayesian analysis also confirmed the lack of difference in implicit probabilistic learning between groups (see Appendix III for details). 


\section{DISCUSSION}

The present thesis summarizes the findings of two studies investigating the effect of TMS methods over the DLPFC (Brodmann 9/46) on WM and implicit probabilistic (statistical) learning along with the investigation of a potential methodological problem in learning and memory studies in clinical and cognitive neuroscience.

More specifically, Study I investigated the effect of an alternative form the rTMS, TBS over the left and right DLPFC on WM-related processes. We administered a WM task, namely the n-back task before and after cTBS, iTBS, or sham stimulation. The results show that participants became faster for the measurement after iTBS or sham stimulation, possibly due to practice. However, cTBS hindered this practice effect and, by that, reduced WM performance. This pattern was observable both when stimulating over the right and left DLPFC. The finding was accompanied by a large effect size.

Study II aimed to investigate how inhibitory stimulation over bilateral DLPFC affects the acquisition and retrieval of probabilistic regularities. To this aim, we administered inhibitory $1 \mathrm{~Hz}$ rTMS bilaterally over the DLPFCs in the process of learning on an implicit probabilistic sequence learning task. We tested the effect of rTMS during learning, as well as $10 \mathrm{~min}, 2 \mathrm{~h}$, and $24 \mathrm{~h}$ after the end of the initial learning. Our results show that the probabilistic knowledge became better $24 \mathrm{~h}$ after the stimulation, suggesting that bilateral disruption of the DLPFCs during learning has a boosting effect on implicit probabilistic learning performance. The difference appeared with a medium effect size.

Study III aimed to test the effects of instruction on implicit probabilistic learning. We instructed two groups of participants to be either fast or accurate while practicing on an implicit probabilistic sequence learning task. After that, participants were tested with instructions to be both fast and accurate at the same time. Results show that although the instructions affected general speed and accuracy (with large effect sizes), the level of probabilistic learning measured by RTs remained similar between groups (performance). However, probabilistic learning was not measurable with accuracy instructions when measured by accuracies. Moreover, a lack of difference was detected in RTs and accuracy measures when tested with the same instructions (competence). 


\section{cTBS over both DLPFCs hinders working memory improvement}

TBS is a prospering method to modulate WM as it operates with theta-gamma coupling (Huang et al., 2005), which is especially involved in WM by realizing coordination during cognitive tasks (Axmacher et al., 2010; Canolty et al., 2006; Niessing et al., 2005). We found a disruptive effect of cTBS on WM-related processes, as unlike iTBS and sham stimulation, it hindered the improvement on the n-back task. The differences were accompanied by large effect sizes. This result is in line with previous studies showing a reduced WM performance after cTBS over the DLPFC (Lee \& D'Esposito, 2012; Schicktanz et al., 2015). Besides the potential depression-like effects caused by the repetitive stimulation, the modulation of theta-gamma oscillations could also play a role in the inhibitory effects of cTBS. This possibility is supported by studies that found cTBS to decrease and iTBS to increase theta-gamma power over the DLPFC (Chung, Lewis, et al., 2017; Chung et al., 2018; Hoy et al., 2016).

Surprisingly, we did not find any effect of the iTBS stimulation compared to the sham stimulation, although enhanced WM performance after iTBS was shown previously (Hoy et al., 2016). It should be noted, however, that iTBS over the DLPFC was mostly associated with altered WM-related oscillatory activity or event-related potentials rather than strong behavioral aftereffects (Chung et al., 2018; Chung, Rogasch, et al., 2017; Hoy et al., 2016). Thus, it is possible that on the electrophysiological level, the iTBS did have an effect, but the changes in WM-related features were not strong enough to manifest as a change in behavioral performance. Another explanation for the lack of iTBS-related effects might be that cTBS methods were shown to lead to more stable cognitive aftereffects than iTBS (Lowe, Manocchio, Safati, \& Hall, 2018). The WM-related electrophysiological aftereffects of cTBS are not described yet, which calls for further investigation on this topic, mostly because of the potentially more stable behavioral changes following cTBS.

We found similar results when stimulating over the left and right DLPFC. Previously, mostly bilateral contribution of the DLPFC or even right DLPFC superiority was found with rTMS in WM performance (Fried et al., 2014; Hamidi et al., 2009; Mottaghy et al., 2002, 2000; Preston et al., 2010; Sandrini et al., 2008; Weigand, Grimm, et al., 2013). On the contrary, neuroimaging results mostly supported left DLPFC superiority for WM (Wager \& Smith, 2003). Studies that found right DLPFC superiority used emotional or spatial WM tasks (Fried et al., 2014; Weigand, Grimm, et al., 2013), which was not the case in our study. Another possibility is that disrupting one hemisphere can cause an imbalance between the two 
hemispheres, leading to reduced performance (Grimm et al., 2008), which would also support the raison d'être of bilateral stimulation methods used in Study II. However, logically, the imbalance theory would also implicate the enhancing nature of iTBS or high-frequency rTMS to lead to an imbalance; thus, these protocols should also result in impaired performance, which is not always the case (Brunoni et al., 2013; Lowe et al., 2018). Nevertheless, our results support that TBS is capable of modulating WM-related processes over both hemispheres.

\section{Disrupting the DLPFCs leads to improved implicit probabilistic learning}

Studies investigating the causal role of DLPFC in acquiring sequential regularities found mainly the facilitating stimulation of the DLPFC to hinder whereas inhibitory stimulation to improve this type of learning (Galea et al., 2010; Pascual-Leone et al., 1996; Smalle et al., 2017). Our results are in line with these findings, as we found better retrieval of probabilistic knowledge $24 \mathrm{~h}$ after the stimulation with medium effect sizes. We went beyond previous results by verifying the disruptive effect of DLPFC also on probabilistic non-adjacent dependencies, and by comparing the effect of rTMS over a longer period. However, a major difference between our results and previous findings is that we did not reveal immediate effect of the disruption of the DLPFC but only $24 \mathrm{~h}$ later. The differences between deterministic and probabilistic learning tasks might explain this result: in non-adjacent second-order probabilistic learning tasks, the to-be-learned information is hidden in noise. Therefore, the building-up of the models about the dependencies might be slower. Nevertheless, it is worth noting that differences in the stimulation parameters such as the intensity of stimulation, the number of pulses, the time of the stimulation relative to learning might cause inconsistencies across studies (Klomjai et al., 2015; Thut \& Pascual-Leone, 2010). Future investigations on the role of the DLPFC in deterministic and probabilistic learning, as well as the effects of different stimulation parameters on them, will help disentangle the inconsistencies in stimulation aftereffects.

Another innovation in our study is that we used bilateral stimulation of the DLPFC. A previous study revealed no effect of TMS on sequence learning, which might have been due to the interhemispheric compensation obscuring the effects (Savic et al., 2017). Another study found hemispheric differences in the effects of TMS on sequence learning (Galea et al., 2010); however, TMS studies proved that lateralization does not necessarily mean that the function is eliminated from the non-dominant hemisphere (Hartwigsen et al., 2010; Mottaghy et al., 2002), thus, that they cannot confound with the results. We hope that future studies would benefit from 
using both unilateral and bilateral stimulation to get a holistic picture of the role of different brain areas and networks in cognitive functions.

\section{The role of DLPFC in working memory and implicit probabilistic learning: what did we learn} from our studies?

Summarizing the results of Study I and Study II, we can claim that the disruptive stimulation of DLPFC could lead to worse WM performance but improved implicit probabilistic learning; thus, inhibiting the DLFPC could improve but also worsen specific learning and memory functions. A growing body of research suggests the existence of competitive neurocognitive networks underlying learning and memory. Such a competitive relationship is theorized to exist between neural circuitry involving the basal ganglia and parts of the medial temporal and the prefrontal lobe (Poldrack \& Packard, 2003). This distinction was primarily associated with non-declarative and declarative memory processes (Squire, 2004). Still, it also harmonizes with conclusions drawn about habitual vs. goal-directed processes (Hardwick, Forrence, Krakauer, \& Haith, 2019; Yin \& Knowlton, 2006), and with the computational theory of model-free and model-based processes (Daw, Niv, \& Dayan, 2005; Smittenaar, FitzGerald, Romei, Wright, \& Dolan, 2013). Although these concepts do not refer to the entirely same learning processes, they share similarities as they reflect more habit-like, incidental vs. more controlled forms of learning.

Prefrontal cortical areas, more specifically, the DLPFC was suggested to play a mediating role between the more voluntary, controlled, and the more incidental forms of learning (e.g., Smittenaar et al., 2013). On an anatomical level, the DLPFC has strong connections with the basal ganglia and the medial temporal lobe (Aggleton, 2012; Draganski et al., 2008), and these connections were found to mediate procedural learning processes, such as implicit probabilistic learning (Bennett et al., 2011). Still, they also play a role in other memory functions such as WM (Chang, Crottaz-Herbette, \& Menon, 2007). Functionally, the DLPFC is strongly associated with executive function and control mechanisms (Barbey, Colom, et al., 2013; MacDonald et al., 2000), that correlate positively with WM performance (McCabe, Roediger, McDaniel, Balota, \& Hambrick, 2010) and declarative memory (Lum, ContiRamsden, Page, \& Ullman, 2012); however, negatively with implicit sequence learning (Nemeth et al., 2013; Smalle et al., 2017; Virag et al., 2015). 
In the framework of competitive neurocognitive networks, our results posit a new theoretical interpretation for the role of DLPFC in probabilistic learning abilities. The DLPFC might have a pivoting role in a more controlled form of learning, such as in accessing long-term memory representations or in higher-level functions supporting these types of learning processes, such as WM (Eppinger et al., 2013; Otto, Skatova, Madlon-Kay, \& Daw, 2014; Scimeca \& Badre, 2012). These processes might be disadvantageous during the incidental learning of new patterns or statistics. Moreover, the already existing representations of statistics hinder the adaptation to new regularities (Kóbor, Horváth, Kardos, Nemeth, \& Janacsek, 2020). If there is only limited access to these controlled processes, then the balance will change in favor of the more incidental forms of learning; thus, the learning of probabilistic regularities will be enhanced. These interpretations give us an answer to why we found improved probabilistic sequence learning performance $24 \mathrm{~h}$ after the inhibitory stimulation of the DLPFC: the stimulation might have interrupted the controlled, top-down information stream during learning, and then, the pure statistical information about the probabilistic regularities consolidated. We propose that the DLPFC might have realized these processes by modulating the activity of the prefrontal-hippocampal circuitry. The above-mentioned competitive relationship between medial temporal regions (hippocampus) and basal ganglia (striatum) was also shown in sequence learning studies, as the activity in the hippocampus decreases in parallel to an increase in the striatal area (Albouy et al., 2015; Albouy et al., 2008). This antagonist relationship was also shown to lead to performance gains after the consolidation of information (Albouy, King, Maquet, \& Doyon, 2013). Thus, we propose that the DLPFC - because of its mediating role - shifted the advantage in this competition in favor of the incidental learning processes.

The findings of Study I can be interpreted similarly to the results of Study II. Although the results of Study I can be viewed as a change in WM itself, we should note that the change was not in WM performance, but in the improvement on WM due to practice. Thus, we can speculate that the disruptive effect of cTBS over the DLPFC might have affected the short-term consolidation of task-specific knowledge, i.e., the learned information about task completion. Following this reasoning, practice effects on cognitive tasks could be interpreted as task-related knowledge helping the individuals improve their performance over multiple testing. This interpretation is supported by the lack of practice effect in neurocognitive disorders (Duff et al., 2007; Ivnik et al., 2000), such as mild cognitive impairment and Alzheimer's Disease where deficits in memory consolidation and in acquiring new information are also detectable (Pace- 
Schott \& Spencer, 2015; Weintraub, Wicklund, \& Salmon, 2012). These consolidation processes might have been modified by the disruption of DLPFC, similarly to how it changed the consolidation of probabilistic regularities in Study II.

An argument against the above interpretation of the findings of Study I is that we did not find practice effects on every difficulty level of the used WM task but only on the more demanding levels. Thus, the changes have to be WM-dependent to some extent, and it might not have been related to general task-related learning abilities, mainly because practice-related effects were described as independent of task difficulty (Reeve \& Lam, 2007). However, the different levels of the n-back task do not only differ in task difficulty but in the measured cognitive abilities (Rac-Lubashevsky \& Kessler, 2016). To decide whether the found effects originate from changes in the short-term consolidation of task-related knowledge or WMspecific processes, TBS or rTMS-induced changes on the practice effect using other cognitive tasks with less WM demand should be investigated. The longitudinal assessment of the dynamics of practice effects could also disclose potential specificities of this phenomenon. The investigation of such task-related changes and the role of DLPFC in such processes awaits future studies.

\section{Instructions do not modify probabilistic representations}

Besides practice effects, the present set of studies dealt with another methodological issue in the research of learning and memory. In Study I and Study II, the effects of TMS over the DLPFC did not reveal itself at the first measurement point. If we had tested the effect of TMS on the measured memory process only once (e.g., immediately following stimulation), we would have obtained null results. This suggests the importance of multiple testing; however, multiple testing raises the question of whether the measurements at different time points, i.e., the measured competence and performance, can differ from each other. As cognitive testing at different time points will be necessarily different in some ways due to the current state of the individuals and the circumstances of the given experimental session, it is crucial to be sure how fragile the memory representations that we are measuring are. In Study III, we investigated implicit probabilistic learning and found that extreme speed and accuracy instructions (simulating different attitudes) do not modify our representations on probabilistic regularities.

We found equal learning performance, both when learning with accuracy or speed instructions. From a theoretical viewpoint, these results underlie the assumption that implicit 
probabilistic learning is a robust learning mechanism, and the acquired representations are stable (Kóbor et al., 2017; Vékony et al., 2019). This means that a similar level of probabilistic knowledge emerges throughout learning when it occurs under different circumstances and with different strategies, at least when using complex probabilistic regularities - with deterministic and more explicit sequences, accuracy instruction was typically found to impair, and/or speed to benefit learning (Barnhoorn, Panzer, Godde, \& Verwey, 2019; Hoyndorf \& Haider, 2009). The more explicit nature of the task used in these studies could explain the difference: increased speed during learning may cover up the explicitness of the task, and ultimately, the task becomes more implicit. The implicitness reduces top-down control, and better learning will be measured. Future studies are warranted to determine how implicitness (or the probabilistic structure) affects the effects of instruction.

From a methodological perspective, it should be noted that a difference was found in RT and accuracy measures: only speed instruction resulted in demonstrable probabilistic sequence learning when measured by accuracies. Contrary to RTs, accuracy can reach a maximum easily. In our study, the participants' accuracy almost reached this maximum when learning with accuracy instruction. Thus, a ceiling effect was obtained that prevented us from measuring learning, although it did occur, as evidenced by the results of the second measurement. These findings raise our attention to a careful approach when evaluating the results obtained by accuracy measures, as it can cause differences in the momentary (measured) performance and the actual competence. In Study I, we found differences in $d$ ' prime, which is based on accuracy; however, it was claimed to be a stable measure of the n-back task (Haatveit et al., 2010). In Study II, our conclusions about implicit probabilistic learning were based on RT scores, which are proven to be a reliable measure of this learning mechanism. Future studies should further explore how RTs or accuracy measurements behave with different learning strategies in other cognitive tasks, as general speed-up and changes in accuracy are typically detectable in various cognitive tasks that require a series of fast decision-making. Until that, although multiple testing seems essential to reveal all potential differences of the tested effect, we highly recommend taking into consideration the possible variances between the competence and the momentary performance when planning learning studies, especially if they involve the evaluation of accuracy measures. 
Table 2. The main questions of the thesis and the results of the studies

The main question to be answered

Results

\begin{tabular}{lll}
\hline Study I & $\begin{array}{l}\text { How does inhibitory and facilitatory TBS } \\
\text { over the left and right DLPFC affect WM } \\
\text { performance? }\end{array}$ & $\begin{array}{l}\text { cTBS hinders improvement on a WM task; iTBS } \\
\text { and sham stimulation both failed to affect WM- } \\
\text { related processes }\end{array}$ \\
Study II & $\begin{array}{l}\text { How does inhibitory rTMS over bilateral } \\
\text { DLPFC affect implicit probabilistic }\end{array}$ & $\begin{array}{l}\text { Inhibitory rTMS over the course of learning leads } \\
\text { to enhanced implicit probabilistic knowledge 24-h }\end{array}$ \\
& sequence learning performance? & later \\
Study III & $\begin{array}{l}\text { How do instructions affect learning and } \\
\text { retrieval of implicit probabilistic } \\
\text { knowledge? }\end{array}$ & $\begin{array}{l}\text { Speed and accuracy instructions do not affect the } \\
\text { acquisition of probabilistic regularities (at least in } \\
\text { RT measures) and lead to similar acquired }\end{array}$ \\
\end{tabular}

\section{Limitations of our studies}

Our studies have limitations that should also be discussed. Limitations of TMS application, in general, might have affected both Study I and Study II. Possibly the most commonly used method to ensure that the measured effects are due to the intended change in neural activity is comparing the effects of verum stimulation to sham stimulation. The sham stimulation methods consist of "simulating" stimulation by rotating the TMS coil away from the scalp by 45-90 degrees or using a sham coil that does not emit real impulses but only imitates the clicking sounds of verum stimulation. Sham protocols aim to create the same belief in participants about the stimulation as if receiving verum stimulation, thus, ensuring blinding. However, sham stimulation cannot control for the sensory side effects (e.g., skin sensation) or the twitches of facial muscles often caused by verum stimulation (Duecker \& Sack, 2015). In Study I, we did not evaluate whether the blinding of participants was successful; thus, it is possible that this factor confounded our results. However, the effects of cTBS found in our study did not only differ from the effects of sham, but also iTBS effects - and it is doubtful that participants can differentiate between the diverse patterns of TBS (cTBS vs. iTBS) concerning their possible cognitive outcomes. In Study II, we measured the level of discomfort and tiredness of participants to assess the potential differences between verum and sham stimulation in terms of the overall experience caused by the stimulation, and we did not find differences between the two experimental groups. However, we only evaluated the experience of the 
participants at the last session, that is, 24-hour after the end of the stimulation. Therefore, we cannot exclude the possibility that the elapsed time between the stimulation and the assessment influenced the precision of the ratings. The reason behind it was that we did not intend to draw the attention of the participants to the (lack) of side effects of the stimulation in order not to bias our results in this sense.

Another possible issue with sham control methods is that we cannot be sure about the regional specificity of the tested effects, that is, whether the stimulation of any other regions would have led to the same outcome, for example, due to induced changes in general arousal or attentional processes. This can only be claimed by active control methods (Duecker \& Sack, 2015). In both Study I, only the $d$ ' was altered due to the stimulation, and in Study II, the effects of stimulation affected only the difference between more and less probable events; thus, it is likely that the effects were due to the involvement of DLPFC in the targeted memory processes. Regional specificity can be further supported by combining TMS with neuroimaging methods, such as electroencephalography (Thut \& Miniussi, 2009) or functional magnetic resonance imaging (Ruff, Driver, \& Bestmann, 2009). Study I and Study II only involved behavioral testing; therefore, a next step to further explore the role of DLPFC in WM and implicit probabilistic learning could be to couple TMS methods with imaging techniques. These methods could reveal the brain activity during and after the stimulation, and also their connection with the tested learning and memory processes.

Study III is limited by the fact that only implicit probabilistic learning was tested. As discussed previously, the explicitness and probabilistic nature of the task could have influenced our results, which makes it essential to test similar processes with other cognitive tasks to reveal how generalizable our findings to a wide range of cognitive processes are.

\section{Future directions}

Although we have answered our primary questions (Table 2), the presented studies opened several new ones. We are planning to continue the started research in multiple directions. First, we have now presented two studies where we successfully modified learning and memory processes with inhibitory TMS. We managed to improve and worsen performance by this type of stimulation, depending on the type of learning. However, the perfect evidence for the competition theory would be to show that the stimulation of the DLPFC can lead to improved or worsened learning performance within the same experiment, with the same 
stimulation parameters. Second, we showed that inhibiting the DLPFC during the acquisition of implicit probabilistic regularities is improved after a 24-hour offline period, possibly through the effect of TMS on the consolidation of the learned information. To further test the assumed role of DLPFC in this type of learning, it would be essential to test if TMS modifies an already acquired knowledge, where consolidation already occurred.

Going a step forward, learning, and retrieving the learned information is not necessarily the end of the learning procedure. Sometimes, we might wish to modify our skills and habits that are already formed. How can we rewire our already existing knowledge? This question is surprisingly neglected in the literature, although we have already started to explore this question (Szegedi-Hallgató et al., 2017). As we have seen that TMS during the learning of implicit probabilistic regularities leads to better-consolidated knowledge, the question is given: can we intervene in this process by manipulating the activity of DLPFC for a more successful rewiring of old habits? Future studies of us will explore this question. 
The presented studies have both theoretical and methodological contributions. From a theoretical perspective, we provided further evidence that the DLPFC plays a role in WMrelated and implicit probabilistic sequence learning processes. As inhibitory TMS over the DLPFC led to a deterioration in WM-related processes, but enhancement in probabilistic learning, thus, our findings provide further evidence that controlled and more incidental forms of memory processes might have an antagonistic relationship. Moreover, we showed that implicit probabilistic sequence learning is robust, resistant form of learning. From a methodological perspective, we underlined the efficacy of cTBS and bilateral stimulation to influence learning and memory processes. We also drew attention to a potential methodological issue in the research of learning and memory by showing how competence and performance can be manipulated in decision-making tasks. We genuinely believe that the present findings contribute to the literature of learning and memory and that they will also help create better TMS-interventions and designs to improve learning abilities also in clinical populations. 


\section{ACKNOWLEDGEMENT}

First of all, I would like to thank Anita Must for her support during my Ph.D. studies. I am deeply grateful to Professor László Vécsei, previous Head of the Department of Neurology, for the opportunity to work at the department and for his continuous support of my research activities. I would like to thank Professor Péter Klivényi, Head of the Department of Neurology, for the opportunity to work at the department.

I am also grateful to Professor Zsigmond Tamás Kincses, and the members of the Neuroimaging Research Group for the opportunity to work at the Neurophysiological Lab and their help during my Ph.D. studies.

I wish to express my gratitude also to Professor Dezső Németh and the members of the Brain, Memory and Language Lab of the Eötvös Loránd University for the fruitful collaborations and their help during my research.

I would like to thank all of my co-authors for their work on our projects and all the valuable knowledge they shared with me.

I am also grateful to my undergraduate supervisors, Gábor Csifcsák and Emese SzegediHallgató, for all the things they taught me about science and their encouragement to pursue a scientific career.

Last but not least, I would like to thank my family for their patience and support during this journey. 


\section{REFERENCES}

Aggleton, J. P. (2012). Multiple anatomical systems embedded within the primate medial temporal lobe: Implications for hippocampal function. Neuroscience and Biobehavioral Reviews, 36(7), 1579-1596. https://doi.org/10.1016/j.neubiorev.2011.09.005

Albouy, G., Fogel, S., King, B. R., Laventure, S., Benali, H., Karni, A., .. Doyon, J. (2015). Maintaining vs. enhancing motor sequence memories: Respective roles of striatal and hippocampal systems. NeuroImage, 108, 423-434. https://doi.org/10.1016/j.neuroimage.2014.12.049

Albouy, G. G., Sterpenich, V., Balteau, E., Vandewalle, G., Desseilles, M., Dang-Vu, T., ... Maquet, P. (2008). Both the hippocampus and striatum are involved in consolidation of motor sequence memory. Neuron, 58(2), 261-272. https://doi.org/10.1016/j.neuron.2008.02.008

Albouy, G., King, B. R., Maquet, P., \& Doyon, J. (2013). Hippocampus and striatum: Dynamics and interaction during acquisition and sleep-related motor sequence memory consolidation. Hippocampus, 23(11), 985-1004. https://doi.org/10.1002/hipo.22183

Axmacher, N., Henseler, M. M., Jensen, O., Weinreich, I., Elger, C. E., \& Fell, J. (2010). Cross-frequency coupling supports multi-item working memory in the human hippocampus. Proceedings of the National Academy of Sciences, 107(7), 3228-3233. https://doi.org/10.1073/pnas.0911531107

Baddeley, A. (1986). Working memory. https://doi.org/10.1002/acp.2350020209

Baddeley, A. (1992). Working memory. Science, 255(5044), 556-559. https://doi.org/10.1126/science.1736359

Bagherzadeh, Y., Khorrami, A., Zarrindast, M. R., Shariat, S. V., \& Pantazis, D. (2016). Repetitive transcranial magnetic stimulation of the dorsolateral prefrontal cortex enhances working memory. Experimental Brain Research, 234(7), 1807-1818. https://doi.org/10.1007/s00221-016-4580-1

Bakulin, I., Zabirova, A., Lagoda, D., Poydasheva, A., Cherkasova, A., Pavlov, N., ... Piradov, M. (2020). Combining HF rTMS over the left DLPFC with concurrent 
cognitive activity for the offline modulation of working memory in healthy volunteers: A proof-of-concept study. Brain Sciences, 10(2), 83.

https://doi.org/10.3390/brainsci10020083

Barbey, A. K., Colom, R., \& Grafman, J. (2013). Dorsolateral prefrontal contributions to human intelligence. Neuropsychologia, 51(7), 1361-1369.

https://doi.org/10.1016/j.neuropsychologia.2012.05.017

Barbey, A. K., Koenigs, M., \& Grafman, J. (2013). Dorsolateral prefrontal contributions to human working memory. Cortex, 49(5), 1195-1205. https://doi.org/10.1016/j.cortex.2012.05.022

Barker, A. T., Jalinous, R., \& Freeston, I. L. (1985). Non-invasive magnetic stimulation of human motor cortex. The Lancet, 325(8437), 1106-1107. https://doi.org/10.1016/S01406736(85)92413-4

Barnhoorn, J. S., Panzer, S., Godde, B., \& Verwey, W. B. (2019). Training motor sequences: Effects of speed and accuracy instructions. Journal of Motor Behavior, 51(5), 540-550. https://doi.org/10.1080/00222895.2018.1528202

Bennett, I. J., Madden, D. J., Vaidya, C. J., Howard, J. H., \& Howard, D. V. (2011). White matter integrity correlates of implicit sequence learning in healthy aging. Neurobiology of Aging, 32(12), 2317.e1-2317.e12. https://doi.org/10.1016/j.neurobiolaging.2010.03.017

Bisiacchi, P. S., Cona, G., Schiff, S., \& Basso, D. (2011). Modulation of a fronto-parietal network in event-based prospective memory: An rTMS study. Neuropsychologia, 49(8), 2225-2232. https://doi.org/10.1016/j.neuropsychologia.2011.05.007

Bo, J., Peltier, S. J., Noll, D. C., \& Seidler, R. D. (2011). Symbolic representations in motor sequence learning. NeuroImage, 54(1), 417-426. https://doi.org/10.1016/j.neuroimage.2010.08.019

Brunoni, A. R., \& Vanderhasselt, M.-A. (2014). Working memory improvement with noninvasive brain stimulation of the dorsolateral prefrontal cortex: A systematic review and meta-analysis. Brain and Cognition, 86, 1-9. https://doi.org/10.1016/j.bandc.2014.01.008 
Brunoni, A. R., Zanao, T. A., Ferrucci, R., Priori, A., Valiengo, L., de Oliveira, J. F., ... Fregni, F. (2013). Bifrontal tDCS prevents implicit learning acquisition in antidepressant-free patients with major depressive disorder. Progress in NeuroPsychopharmacology and Biological Psychiatry, 43, 146-150.

https://doi.org/10.1016/j.pnpbp.2012.12.019

Calero, M. D., \& Navarro, E. (2004). Relationship between plasticity, mild cognitive impairment and cognitive decline. Archives of Clinical Neuropsychology, 19(5), 653660. https://doi.org/10.1016/j.acn.2003.08.008

Canolty, R. T., Edwards, E., Dalal, S. S., Soltani, M., Nagarajan, S. S., Kirsch, H. E., ... Knight, R. T. (2006). High gamma power is phase-locked to theta oscillations in human neocortex. Science, 313(5793), 1626-1628. https://doi.org/10.1126/science.1128115

Case, R., Kurland, D. M., \& Goldberg, J. (1982). Operational efficiency and the growth of short-term memory span. Journal of Experimental Child Psychology, 33(3), 386-404. https://doi.org/10.1016/0022-0965(82)90054-6

Chang, C., Crottaz-Herbette, S., \& Menon, V. (2007). Temporal dynamics of basal ganglia response and connectivity during verbal working memory. NeuroImage, 34(3), 12531269. https://doi.org/10.1016/j.neuroimage.2006.08.056

Chung, S. W., Lewis, B. P., Rogasch, N. C., Saeki, T., Thomson, R. H., Hoy, K. E., ... Fitzgerald, P. B. (2017). Demonstration of short-term plasticity in the dorsolateral prefrontal cortex with theta burst stimulation: A TMS-EEG study. Clinical Neurophysiology, 128(7), 1117-1126. https://doi.org/10.1016/j.clinph.2017.04.005

Chung, S. W., Rogasch, N. C., Hoy, K. E., \& Fitzgerald, P. B. (2018). The effect of single and repeated prefrontal intermittent theta burst stimulation on cortical reactivity and working memory. Brain Stimulation, 11(3), 566-574. https://doi.org/10.1016/j.brs.2018.01.002

Chung, S. W., Rogasch, N. C., Hoy, K. E., Sullivan, C. M., Cash, R. F. H., \& Fitzgerald, P. B. (2017). Impact of different intensities of intermittent theta burst stimulation on the cortical properties during TMS-EEG and working memory performance. Human Brain Mapping, (1072057), 157917. https://doi.org/10.1002/hbm.23882

Cohen, J. (1992). A power primer. Psychological Bulletin, 112(1), 155-159. 
https://doi.org/10.1037/0033-2909.112.1.155

Cohen, J. R., \& Poldrack, R. A. (2008). Automaticity in motor sequence learning does not impair response inhibition. Psychonomic Bulletin \& Review, 15(1), 108-115. https://doi.org/10.3758/PBR.15.1.108

Conway, A. R. A., Kane, M. J., Bunting, M. F., Hambrick, D. Z., Wilhelm, O., \& Engle, R. W. (2005). Working memory span tasks: A methodological review and user's guide. Psychonomic Bulletin and Review, 12(5), 769-786. https://doi.org/10.3758/BF03196772

Daw, N. D., Niv, Y., \& Dayan, P. (2005). Uncertainty-based competition between prefrontal and dorsolateral striatal systems for behavioral control. Nature Neuroscience, $8(12)$, 1704-1711. https://doi.org/10.1038/nn1560

Demeter, E. (2016). Enhancing cognition with theta burst stimulation. Current Behavioral Neuroscience Reports, 3(2), 87-94. https://doi.org/10.1007/s40473-016-0072-7

Deng, Z. De, Lisanby, S. H., \& Peterchev, A. V. (2013). Electric field depth-focality tradeoff in transcranial magnetic stimulation: Simulation comparison of 50 coil designs. Brain Stimulation, 6(1), 1-13. https://doi.org/10.1016/j.brs.2012.02.005

DeSousa, E. A., Albert, R. H., \& Kalman, B. (2002). Cognitive impairments in multiple sclerosis: A review. American Journal of Alzheimer's Disease \& Other Dementiasr, 17(1), 23-29. https://doi.org/10.1177/153331750201700104

Doyon, J., Bellec, P., Amsel, R., Penhune, V., Monchi, O., Carrier, J., ... Benali, H. (2009, April 12). Contributions of the basal ganglia and functionally related brain structures to motor learning. Behavioural Brain Research, Vol. 199, pp. 61-75. https://doi.org/10.1016/j.bbr.2008.11.012

Draganski, B., Kherif, F., Klöppel, S., Cook, P. A., Alexander, D. C., Parker, G. J. M., ... Frackowiak, R. S. J. (2008). Evidence for segregated and integrative connectivity patterns in the human basal ganglia. Journal of Neuroscience, 28(28), 7143-7152. https://doi.org/10.1523/JNEUROSCI.1486-08.2008

Duecker, F., \& Sack, A. T. (2015). Rethinking the role of sham TMS. Frontiers in Psychology, 6, 210. https://doi.org/10.3389/fpsyg.2015.00210 
Duff, K., Beglinger, L. J., Schultz, S. K., Moser, D. J., McCaffrey, R. J., Haase, R. F., ... Paulsen, J. S. (2007). Practice effects in the prediction of long-term cognitive outcome in three patient samples: A novel prognostic index. Archives of Clinical Neuropsychology, 22(1), 15-24. https://doi.org/10.1016/j.acn.2006.08.013

Duff, K., Callister, C., Dennett, K., \& Tometich, D. (2012). Practice effects: A unique cognitive variable. Clinical Neuropsychologist, 26(7), 1117-1127. https://doi.org/10.1080/13854046.2012.722685

Düzel, E., Penny, W. D., \& Burgess, N. (2010). Brain oscillations and memory. Current Opinion in Neurobiology, 20(2), 245-257. https://doi.org/10.1016/j.conb.2010.01.004

Eldaief, M. C., Halko, M. A., Buckner, R. L., \& Pascual-Leone, A. (2011). Transcranial magnetic stimulation modulates the brain's intrinsic activity in a frequency-dependent manner. Proceedings of the National Academy of Sciences of the United States of America, 108(52), 21229-21234. https://doi.org/10.1073/pnas.1113103109

Engle, R. W., Laughlin, J. E., Tuholski, S. W., \& Conway, A. R. A. (1999). Working memory, short-term memory, and general fluid intelligence: A latent-variable approach. Journal of Experimental Psychology: General, 128(3), 309-331. https://doi.org/10.1037/0096-3445.128.3.309

Eppinger, B., Walter, M., Heekeren, H. R., \& Li, S. C. (2013). Of goals and habits: Agerelated and individual differences in goal-directed decision-making. Frontiers in Neuroscience, 7, 253. https://doi.org/10.3389/fnins.2013.00253

Fletcher, P. C., Zafiris, O., Frith, C. D., Honey, R. A. E., Corlett, P. R., Zilles, K., \& Fink, G. R. (2005). On the benefits of not trying: Brain activity and connectivity reflecting the interactions of explicit and implicit sequence learning. Cerebral Cortex, 15(7), 10021015. https://doi.org/10.1093/cercor/bhh201

Fox, C. J., Mueller, S. T., Gray, H. M., Raber, J., \& Piper, B. J. (2013). Evaluation of a shortform of the Berg Card Sorting Test. PLoS ONE, 8(5), e63885. https://doi.org/10.1371/journal.pone.0063885

Frensch, P. A., \& Miner, C. S. (1994). Effects of presentation rate and individual differences in short-term memory capacity on an indirect measure of serial learning. Memory \& 
Cognition, 22(1), 95-110. https://doi.org/10.3758/BF03202765

Fried, P. J., Rushmore, R. J., Moss, M. B., Valero-Cabré, A., \& Pascual-Leone, A. (2014). Causal evidence supporting functional dissociation of verbal and spatial working memory in the human dorsolateral prefrontal cortex. European Journal of Neuroscience, 39(11), 1973-1981. https://doi.org/10.1111/ejn.12584

Galea, J. M., Albert, N. B., Ditye, T., \& Miall, R. C. (2010). Disruption of the dorsolateral prefrontal cortex facilitates the consolidation of procedural skills. Journal of Cognitive Neuroscience, 22(6), 1158-1164. https://doi.org/10.1162/jocn.2009.21259

Gaudeau-Bosma, C., Moulier, V., Allard, A.-C., Sidhoumi, D., Bouaziz, N., Braha, S., ... Januel, D. (2013). Effect of two weeks of rTMS on brain activity in healthy subjects during an n-back task: A randomized double blind study. Brain Stimulation, 6(4), 569575. https://doi.org/10.1016/j.brs.2012.10.009

Gevins, A., \& Cutillo, B. (1993). Spatiotemporal dynamics of component processes in human working memory. Electroencephalography and Clinical Neurophysiology, 87(3), 128143. https://doi.org/10.1016/0013-4694(93)90119-G

Gheysen, F., Van Opstal, F., Roggeman, C., Van Waelvelde, H., \& Fias, W. (2011). The neural basis of implicit perceptual sequence learning. Frontiers in Human Neuroscience, 5. https://doi.org/10.3389/fnhum.2011.00137

Grimm, S., Beck, J., Schuepbach, D., Hell, D., Boesiger, P., Bermpohl, F., .. Northoff, G. (2008). Imbalance between left and right dorsolateral prefrontal cortex in major depression is linked to negative emotional judgment: An fMRI study in severe major depressive disorder. Biological Psychiatry, 63(4), 369-376.

https://doi.org/10.1016/j.biopsych.2007.05.033

Guse, B., Falkai, P., Gruber, O., Whalley, H., Gibson, L., Hasan, A., ... Wobrock, T. (2013). The effect of long-term high frequency repetitive transcranial magnetic stimulation on working memory in schizophrenia and healthy controls-A randomized placebocontrolled, double-blind fMRI study. Behavioural Brain Research, 237(1), 300-307. https://doi.org/10.1016/j.bbr.2012.09.034

Haatveit, B. C., Sundet, K., Hugdahl, K., Ueland, T., Melle, I., \& Andreassen, O. A. (2010). 
The validity of $d$ prime as a working memory index: results from the "Bergen $n$-back task”. Journal of Clinical and Experimental Neuropsychology, 32(8), 871-880. https://doi.org/10.1080/13803391003596421

Hallett, M. (2000). Transcranial magnetic stimulation and the human brain. Nature, 406(6792), 147-150. https://doi.org/10.1038/35018000

Hamidi, M., Tononi, G., \& Postle, B. R. (2009). Evaluating the role of prefrontal and parietal cortices in memory-guided response with repetitive transcranial magnetic stimulation. Neuropsychologia, 47(2), 295-302. https://doi.org/10.1016/j.neuropsychologia.2008.08.026

Hardwick, R. M., Forrence, A. D., Krakauer, J. W., \& Haith, A. M. (2019). Time-dependent competition between goal-directed and habitual response preparation. Nature Human Behaviour, 3(12), 1252-1262. https://doi.org/10.1038/s41562-019-0725-0

Hartwigsen, G., Baumgaertner, A., Price, C. J., Koehnke, M., Ulmer, S., \& Siebner, H. R. (2010). Phonological decisions require both the left and right supramarginal gyri. Proceedings of the National Academy of Sciences of the United States of America, 107(38), 16494-16499. https://doi.org/10.1073/pnas.1008121107

Heekeren, H. R., Marrett, S., Ruff, D. A., Bandettini, P. A., \& Ungerleider, L. G. (2006). Involvement of human left dorsolateral prefrontal cortex in perceptual decision making is independent of response modality. Proceedings of the National Academy of Sciences of the United States of America, 103(26), 10023-10028. https://doi.org/10.1073/pnas.0603949103

Heideman, S. G., van Ede, F., \& Nobre, A. C. (2018). Temporal alignment of anticipatory motor cortical beta lateralisation in hidden visual-motor sequences. European Journal of Neuroscience, 48(8), 2684-2695. https://doi.org/10.1111/ejn.13700

Howard, J. H., \& Howard, D. V. (1997). Age differences in implicit learning of higher order dependencies in serial patterns. Psychology and Aging, 12(4), 634-656. https://doi.org/10.1037/0882-7974.12.4.634

Howard, D. V., Howard, J. H., Japikse, K., DiYanni, C., Thompson, A., \& Somberg, R. (2004). Implicit sequence learning: Effects of level of structure, adult age, and extended 
practice. Psychology and Aging, 19(1), 79-92. https://doi.org/10.1037/0882-

7974.19.1.79

Hoy, K. E., Bailey, N., Michael, M., Fitzgibbon, B., Rogasch, N. C., Saeki, T., \& Fitzgerald, P. B. (2016). Enhancement of working memory and task-related oscillatory activity following intermittent theta burst stimulation in healthy controls. Cerebral Cortex, 1-11. https://doi.org/10.1093/cercor/bhv193

Hoyndorf, A., \& Haider, H. (2009). The "Not Letting Go" phenomenon: Accuracy instructions can impair behavioral and metacognitive effects of implicit learning processes. Psychological Research, 73(5), 695-706. https://doi.org/10.1007/s00426-0080180-4

Huang, Y.-Z. Z., Edwards, M. J., Rounis, E., Bhatia, K. P., \& Rothwell, J. C. (2005). Theta burst stimulation of the human motor cortex. Neuron, 45(2), 201-206. https://doi.org/10.1016/j.neuron.2004.12.033

Hulst, H. E., Goldschmidt, T., Nitsche, M. A., De Wit, S. J., Van Den Heuvel, O. A., Barkhof, F., ... Geurts, J. J. G. (2017). RTMS affects working memory performance, brain activation and functional connectivity in patients with multiple sclerosis. Journal of Neurology, Neurosurgery and Psychiatry, 88(5), 386-394. https://doi.org/10.1136/jnnp2016-314224

Ivnik, R. J., Smith, G. E., Petersen, R. C., Boeve, B. F., Kokmen, E., \& Tangalos, E. G. (2000). Diagnostic accuracy of four approaches to interpreting neuropsychological test data. Neuropsychology, 14(2), 163-177. https://doi.org/10.1037/0894-4105.14.2.163

Jacoby, L. L. (1991). A process dissociation framework: Separating automatic from intentional uses of memory. Journal of Memory and Language, 30(5), 513-541. https://doi.org/10.1016/0749-596X(91)90025-F

Janacsek, K., \& Nemeth, D. (2013). Implicit sequence learning and working memory: Correlated or complicated? Cortex, 49(8), 2001-2006. https://doi.org/10.1016/j.cortex.2013.02.012

Janacsek, K., \& Nemeth, D. (2015). The puzzle is complicated: when should working memory be related to implicit sequence learning, and when should it not? (Response to 
Martini et al.). Cortex, 64(411-412). https://doi.org/10.1016/j.cortex.2014.07.020

Janacsek, K., Shattuck, K. F., Tagarelli, K. M., Lum, J. A. G., Turkeltaub, P. E., \& Ullman, M. T. (2020). Sequence learning in the human brain: A functional neuroanatomical metaanalysis of serial reaction time studies. NeuroImage, 207, 116387. https://doi.org/10.1016/j.neuroimage.2019.116387

Kaller, C. P., Rahm, B., Spreer, J., Weiller, C., \& Unterrainer, J. M. (2011). Dissociable contributions of left and right dorsolateral prefrontal cortex in planning. Cerebral Cortex, 21(2), 307-317. https://doi.org/10.1093/cercor/bhq096

Kaufman, S. B., DeYoung, C. G., Gray, J. R., Jiménez, L., Brown, J., \& Mackintosh, N. (2010). Implicit learning as an ability. Cognition, 116(3), 321-340. https://doi.org/10.1016/j.cognition.2010.05.011

Kirchner, W. K. (1958). Age differences in short-term retention of rapidly changing information. Journal of Experimental Psychology, 55(4), 352-358. https://doi.org/10.1037/h0043688

Kirova, A. M., Bays, R. B., \& Lagalwar, S. (2015). Working memory and executive function decline across normal aging, mild cognitive impairment, and Alzheimer's Disease. BioMed Research International, 2015. https://doi.org/10.1155/2015/748212

Klomjai, W., Katz, R., \& Lackmy-Vallée, A. (2015). Basic principles of transcranial magnetic stimulation (TMS) and repetitive TMS (rTMS). Annals of Physical and Rehabilitation Medicine, 58(4), 208-213. https://doi.org/10.1016/j.rehab.2015.05.005

Ko, J. H., Monchi, O., Ptito, A., Bloomfield, P., Houle, S., \& Strafella, A. P. (2008). Theta burst stimulation-induced inhibition of dorsolateral prefrontal cortex reveals hemispheric asymmetry in striatal dopamine release during a set-shifting task - A TMS[11C]raclopride PET study. European Journal of Neuroscience, 28(10), 2147-2155. https://doi.org/10.1111/j.1460-9568.2008.06501.x

Kóbor, A., Horváth, K., Kardos, Z., Nemeth, D., \& Janacsek, K. (2020). Perceiving structure in unstructured stimuli: Implicitly acquired prior knowledge impacts the processing of unpredictable transitional probabilities. Cognition, 104413. https://doi.org/10.1016/j.cognition.2020.104413 
Kóbor, A., Janacsek, K., Takács, A., Nemeth, D., Kobor, A., Janacsek, K., ... Nemeth, D. (2017). Statistical learning leads to persistent memory: Evidence for one-year consolidation. Scientific Reports, 7(1), 1-10. https://doi.org/10.1038/s41598-017-008073

Kóbor, A., Takács, Á., Kardos, Z., Janacsek, K., Horváth, K., Csépe, V., \& Nemeth, D. (2018). ERPs differentiate the sensitivity to statistical probabilities and the learning of sequential structures during procedural learning. Biological Psychology, 135(April), 180-193. https://doi.org/10.1016/j.biopsycho.2018.04.001

Kujirai, T., Caramia, M. D., Rothwell, J. C., Day, B. L., Thompson, P. D., Ferbert, A., ... Marsden, C. D. (1993). Corticocortical inhibition in human motor cortex. The Journal of Physiology, 471(1), 501-519. https://doi.org/10.1113/jphysiol.1993.sp019912

Lee, T. G., \& D'Esposito, M. (2012). The dynamic nature of top-down signals originating from prefrontal cortex: a combined fMRI-TMS study. Journal of Neuroscience, 32(44), 15458-15466. https://doi.org/10.1523/JNEUROSCI.0627-12.2012

Lieberman, M. D. (2000). Intuition: A social cognitive neuroscience approach. Psychological Bulletin, 126(1), 109-137. https://doi.org/10.1037/0033-2909.126.1.109

Lisman, J. (2010). Working memory: The importance of theta and gamma oscillations. Current Biology, 20(11), R490-R492. https://doi.org/10.1016/j.cub.2010.04.011

Lisman, J., \& Jensen, O. (2013). The theta-gamma neural code. Neuron, 77(6), 1002-1016. https://doi.org/10.1016/j.neuron.2013.03.007

Lowe, C. J., Manocchio, F., Safati, A. B., \& Hall, P. A. (2018). The effects of theta burst stimulation (TBS) targeting the prefrontal cortex on executive functioning: A systematic review and meta-analysis. Neuropsychologia, 111, 344-359. https://doi.org/10.1016/j.neuropsychologia.2018.02.004

Luber, B., \& Lisanby, S. H. (2014). Enhancement of human cognitive performance using transcranial magnetic stimulation (TMS). NeuroImage, 85, 961-970. https://doi.org/10.1016/j.neuroimage.2013.06.007

Lum, J. A. G., Conti-Ramsden, G., Page, D., \& Ullman, M. T. (2012). Working, declarative 
and procedural memory in specific language impairment. Cortex, 48(9), 1138-1154. https://doi.org/10.1016/j.cortex.2011.06.001

MacDonald, A. W., Cohen, J. D., Stenger, V. A., \& Carter, C. S. (2000). Dissociating the role of the dorsolateral prefrontal and anterior cingulate cortex in cognitive control. Science, 288(5472), 1835-1838. https://doi.org/10.1126/science.288.5472.1835

Mackey, W. E., Devinsky, O., Doyle, W. K., Meager, M. R., \& Curtis, C. E. (2016). Human dorsolateral prefrontal cortex is not necessary for spatial working memory. Journal of Neuroscience, 36(10), 2847-2856. https://doi.org/10.1523/JNEUROSCI.3618-15.2016

Martini, M., Sachse, P., Furtner, M. R., \& Gaschler, R. (2015). Why should working memory be related to incidentally learned sequence structures? Cortex, 64, 407-410. https://doi.org/10.1016/j.cortex.2014.05.016

McCabe, D. P., Roediger, H. L., McDaniel, M. A., Balota, D. A., \& Hambrick, D. Z. (2010). The relationship between working memory capacity and executive functioning: Evidence for a common executive attention construct. Neuropsychology, 24(2), 222-243. https://doi.org/10.1037/a0017619

Merton, P. A., \& Morton, H. B. (1980). Stimulation of the cerebral cortex in the intact human subject. Nature, 285(5762), 227. https://doi.org/10.1038/285227a0

Mottaghy, F. M., Döring, T., Müller-Gärtner, H.-W. W., Töpper, R., \& Krause, B. J. (2002). Bilateral parieto-frontal network for verbal working memory: An interference approach using repetitive transcranial magnetic stimulation (rTMS). European Journal of Neuroscience, 16(8), 1627-1632. https://doi.org/10.1046/j.1460-9568.2002.02209.x

Mottaghy, F. M., Krause, B. J., Kemna, L. J., Töpper, R., Tellmann, L., Beu, M., ... MüllerGärtner, H.-W. (2000). Modulation of the neuronal circuitry subserving working memory in healthy human subjects by repetitive transcranial magnetic stimulation. Neuroscience Letters, 280(3), 167-170. https://doi.org/10.1016/S0304-3940(00)00798-9

Mull, B. R., \& Seyal, M. (2001). Transcranial magnetic stimulation of left prefrontal cortex impairs working memory. Clinical Neurophysiology, 112(9), 1672-1675. https://doi.org/10.1016/S1388-2457(01)00606-X 
Munoz, D. P., \& Everling, S. (2004). Look away: the anti-saccade task and the voluntary control of eye movement. Nature Reviews. Neuroscience, 5(3), 218-228. https://doi.org/10.1038/nrn1345

Nemeth, D., Janacsek, K., Balogh, V., Londe, Z., Mingesz, R., Fazekas, M., ... Vetro, A. (2010). Learning in autism: Implicitly superb. PLoS ONE, 5(7), 1-7. https://doi.org/10.1371/journal.pone.0011731

Nemeth, D., Janacsek, K., Polner, B., \& Kovacs, Z. A. (2013). Boosting human learning by hypnosis. Cerebral Cortex, 23(4), 801-805. https://doi.org/10.1093/cercor/bhs068

Niessing, J., Ebisch, B., Schmidt, K. E., Niessing, M., Singer, W., \& Galuske, R. A. W. (2005). Neuroscience: Hemodynamic signals correlate tightly with synchronized gamma oscillations. Science, 309(5736), 948-951. https://doi.org/10.1126/science.1110948

Nissen, M. J., \& Bullemer, P. (1987). Attentional requirements of learning: Evidence from performance measures. Cognitive Psychology, 19(1), 1-32. https://doi.org/10.1016/00100285(87)90002-8

Noda, Y., Silverstein, W. K., Barr, M. S., Vila-Rodriguez, F., Downar, J., Rajji, T. K., ... Blumberger, D. M. (2015). Neurobiological mechanisms of repetitive transcranial magnetic stimulation of the dorsolateral prefrontal cortex in depression: A systematic review. Psychological Medicine, 45(16), 3411-3432. https://doi.org/10.1017/S0033291715001609

Nyffeler, T., Wurtz, P., Lüscher, H. R., Hess, C. W., Senn, W., Pflugshaupt, T., ... Müri, R. M. (2006). Repetitive TMS over the human oculomotor cortex: Comparison of 1-Hz and theta burst stimulation. Neuroscience Letters, 409(1), 57-60. https://doi.org/10.1016/j.neulet.2006.09.011

Oehrn, C. R., Fell, J., Baumann, C., Rosburg, T., Ludowig, E., Kessler, H., ... Axmacher, N. (2018). Direct electrophysiological evidence for prefrontal control of hippocampal processing during voluntary forgetting. Current Biology, 28(18), 3016-3022.e4. https://doi.org/10.1016/j.cub.2018.07.042

Otto, A. R., Raio, C. M., Chiang, A., Phelps, E. A., \& Daw, N. D. (2013). Working-memory capacity protects model-based learning from stress. Proceedings of the National 
Academy of Sciences of the United States of America, 110(52), 20941-20946. https://doi.org/10.1073/pnas.1312011110

Otto, A. R., Skatova, A., Madlon-Kay, S., \& Daw, N. D. (2014). Cognitive control predicts use of model-based reinforcement learning. Journal of Cognitive Neuroscience, 27(2), 319-333. https://doi.org/10.1162/jocn_a_00709

Owen, A. M., McMillan, K. M., Laird, A. R., \& Bullmore, E. (2005). N-back working memory paradigm: A meta-analysis of normative functional neuroimaging studies. Human Brain Mapping, 25(1), 46-59. https://doi.org/10.1002/hbm.20131

Pace-Schott, E. F., \& Spencer, R. M. C. (2015). Sleep-dependent memory consolidation in healthy aging and mild cognitive impairment. Current Topics in Behavioral Neurosciences, 25, 307-330. https://doi.org/10.1007/7854_2014_300

Pascual-Leone, A., Wassermann, E. M., Grafman, J., \& Hallett, M. (1996). The role of the dorsolateral prefrontal cortex in implicit procedural learning. Experimental Brain Research, 107(3), 479-485. https://doi.org/10.1007/BF00230427

Patel, R., Silla, F., Pierce, S., Theule, J., \& Girard, T. A. (2020). Cognitive functioning before and after repetitive transcranial magnetic stimulation (rTMS): A quantitative metaanalysis in healthy adults. Neuropsychologia, 141 . https://doi.org/10.1016/j.neuropsychologia.2020.107395

Paulus, W. (2011). Transcranial electrical stimulation (tES - tDCS; tRNS, tACS) methods. Neuropsychological Rehabilitation, 21(5), 602-617. https://doi.org/10.1080/09602011.2011.557292

Peinemann, A., Reimer, B., Löer, C., Quartarone, A., Münchau, A., Conrad, B., \& Siebner, H. R. (2004). Long-lasting increase in corticospinal excitability after 1800 pulses of subthreshold $5 \mathrm{~Hz}$ repetitive TMS to the primary motor cortex. Clinical Neurophysiology, 115(7), 1519-1526. https://doi.org/10.1016/j.clinph.2004.02.005

Peirce, J. W. (2007). PsychoPy-Psychophysics software in Python. Journal of Neuroscience Methods, 162(1-2), 8-13. https://doi.org/10.1016/j.jneumeth.2006.11.017

Poldrack, R. A., \& Packard, M. G. (2003). Competition among multiple memory systems: 
Converging evidence from animal and human brain studies. Neuropsychologia, 41(3), 245-251. https://doi.org/10.1016/S0028-3932(02)00157-4

Poldrack, R. A., Sabb, F. W., Foerde, K., Tom, S. M., Asarnow, R. F., Bookheimer, S. Y., \& Knowlton, B. J. (2005). The neural correlates of motor skill automaticity. Journal of Neuroscience, 25(22), 5356-5364. https://doi.org/10.1523/JNEUROSCI.3880-04.2005

Preston, G., Anderson, E., Silva, C., Goldberg, T., \& Wassermann, E. M. (2010). Effects of $10 \mathrm{~Hz}$ rTMS on the neural efficiency of working memory. Journal of Cognitive Neuroscience, 22(3), 447-456. https://doi.org/10.1162/jocn.2009.21209

Rac-Lubashevsky, R., \& Kessler, Y. (2016). Decomposing the n-back task: An individual differences study using the reference-back paradigm. Neuropsychologia, 90, 190-199. https://doi.org/10.1016/j.neuropsychologia.2016.07.013

Reeve, C. L., \& Lam, H. (2007). The relation between practice effects, test-taker characteristics and degree of g-saturation. International Journal of Testing, 7(2), 225242. https://doi.org/10.1080/15305050701193595

Remillard, G. (2008). Implicit learning of second-, third-, and fourth-order adjacent and nonadjacent sequential dependencies. Quarterly Journal of Experimental Psychology, 61(3), 400-424. https://doi.org/10.1080/17470210701210999

Rieckmann, A., Fischer, H., \& Bäckman, L. (2010). Activation in striatum and medial temporal lobe during sequence learning in younger and older adults: Relations to performance. NeuroImage, 50(3), 1303-1312. https://doi.org/10.1016/j.neuroimage.2010.01.015

Riley, J. D., Moore, S., Cramer, S. C., \& Lin, J. J. (2011). Caudate atrophy and impaired frontostriatal connections are linked to executive dysfunction in temporal lobe epilepsy. Epilepsy and Behavior, 21(1), 80-87. https://doi.org/10.1016/j.yebeh.2011.03.013

Rose, M., Haider, H., Salari, N., \& Buchel, C. (2011). Functional dissociation of hippocampal mechanism during implicit learning based on the domain of associations. Journal of Neuroscience, 31(39), 13739-13745. https://doi.org/10.1523/jneurosci.3020-11.2011

Röttger, E., Haider, H., Zhao, F., \& Gaschler, R. (2017). Implicit sequence learning despite 
multitasking: the role of across-task predictability. Psychological Research, 83(3), 526543. https://doi.org/10.1007/s00426-017-0920-4

Ruff, C. C., Driver, J., \& Bestmann, S. (2009). Combining TMS and fMRI: From "virtual lesions" to functional-network accounts of cognition. Cortex, 45(9), 1043-1049. https://doi.org/10.1016/j.cortex.2008.10.012

Sandrini, M., Cappa, S. F., Rossi, S., Rossini, P. M., \& Miniussi, C. (2003). The role of prefrontal cortex in verbal episodic memory: rTMS evidence. Journal of Cognitive Neuroscience, 15(6), 855-861. https://doi.org/10.1162/089892903322370771

Sandrini, M., Rossini, P. M., \& Miniussi, C. (2008). Lateralized contribution of prefrontal cortex in controlling task-irrelevant information during verbal and spatial working memory tasks: rTMS evidence. Neuropsychologia, 46(7), 2056-2063. https://doi.org/10.1016/j.neuropsychologia.2008.02.003

Sandrini, M., Umiltà, C., \& Rusconi, E. (2011). The use of transcranial magnetic stimulation in cognitive neuroscience: A new synthesis of methodological issues. Neuroscience and Biobehavioral Reviews, 35(3), 516-536. https://doi.org/10.1016/j.neubiorev.2010.06.005

Sato, H., Yahata, N., Funane, T., Takizawa, R., Katura, T., Atsumori, H., ... Kasai, K. (2013). A NIRS-fMRI investigation of prefrontal cortex activity during a working memory task. NeuroImage, 83, 158-173. https://doi.org/10.1016/j.neuroimage.2013.06.043

Savic, B., Cazzoli, D., Müri, R., \& Meier, B. (2017). No effects of transcranial DLPFC stimulation on implicit task sequence learning and consolidation. Scientific Reports, 7(1), 9649. https://doi.org/10.1038/s41598-017-10128-0

Schicktanz, N., Fastenrath, M., Milnik, A., Spalek, K., Auschra, B., Nyffeler, T., ... Schwegler, K. (2015). Continuous theta burst stimulation over the left dorsolateral prefrontal cortex decreases medium load working memory performance in healthy humans. PLoS ONE, 10(3), e0120640. https://doi.org/10.1371/journal.pone.0120640

Schneider, S. A., Wilkinson, L., Bhatia, K. P., Henley, S. M. D., Rothwell, J. C., Tabrizi, S. J., \& Jahanshahi, M. (2010). Abnormal explicit but normal implicit sequence learning in premanifest and early Huntington's disease. Movement Disorders, 25(10), 1343-1349. https://doi.org/10.1002/mds.22692 
Schwarb, H., \& Schumacher, E. H. (2009). Neural evidence of a role for spatial response selection in the learning of spatial sequences. Brain Research, 1247, 114-125. https://doi.org/10.1016/j.brainres.2008.09.097

Scimeca, J. M., \& Badre, D. (2012). Striatal contributions to declarative memory retrieval. Neuron, 75(3), 380-392. https://doi.org/10.1016/j.neuron.2012.07.014

Shields, J., Mock, J., Devier, D., \& Foundas, A. (2017). Unilateral repetitive transcranial magnetic stimulation differentially affects younger and older adults completing a verbal working memory task. Journal of the Neurological Sciences, 384, 15-20. https://doi.org/10.1016/j.jns.2017.10.021

Siegel, M., Warden, M. R., \& Miller, E. K. (2009). Phase-dependent neuronal coding of objects in short-term memory. Proceedings of the National Academy of Sciences of the United States of America, 106(50), 21341-21346. https://doi.org/10.1073/pnas.0908193106

Silvanto, J., Cattaneo, Z., Battelli, L., \& Pascual-Leone, A. (2008). Baseline cortical excitability determines whether TMS disrupts or facilitates behavior. Journal of Neurophysiology, 99(5), 2725-2730. https://doi.org/10.1152/jn.01392.2007

Simon, J. R., Vaidya, C. J., Howard, J. H., \& Howard, D. V. (2012). The effects of aging on the neural basis of implicit associative learning in a probabilistic triplets learning task. Journal of Cognitive Neuroscience, 24(2), 451-463. https://doi.org/10.1162/jocn_a_00116

Smalle, E. H. M., Panouilleres, M., Szmalec, A., \& Möttönen, R. (2017). Language learning in the adult brain: Disrupting the dorsolateral prefrontal cortex facilitates word-form learning. Scientific Reports, 7(1), 13966. https://doi.org/10.1038/s41598-017-14547-x

Smittenaar, P., FitzGerald, T. H. B., Romei, V., Wright, N. D., \& Dolan, R. J. (2013). Disruption of dorsolateral prefrontal cortex decreases model-based in favor of modelfree control in humans. Neuron, 80(4), 914-919. https://doi.org/10.1016/j.neuron.2013.08.009

Soderstrom, N. C., \& Bjork, R. A. (2015). Learning versus performance: An integrative review. Perspectives on Psychological Science, 10(2), 176-199. 
https://doi.org/10.1177/1745691615569000

Song, S., Howard, J. H., \& Howard, D. V. (2007). Sleep does not benefit probabilistic motor sequence learning. Journal of Neuroscience, 27(46), 12475-12483. https://doi.org/10.1523/JNEUROSCI.2062-07.2007

Squire, L. R. (2004). Memory systems of the brain: A brief history and current perspective. Neurobiology of Learning and Memory, 82(3), 171-177. https://doi.org/10.1016/j.nlm.2004.06.005

Stillman, C. M., Gordon, E. M., Simon, J. R., Vaidya, C. J., Howard, D. V., \& Howard, J. H. (2013). Caudate resting connectivity predicts implicit probabilistic sequence learning. Brain Connectivity, 3(6), 601-610. https://doi.org/10.1089/brain.2013.0169

Stout, J. C., Paulsen, J. S., Queller, S., Solomon, A. C., Whitlock, K. B., Campbell, J. C., ... Aylward, E. H. (2011). Neurocognitive signs in prodromal Huntington Disease. Neuropsychology, 25(1), 1-14. https://doi.org/10.1037/a0020937

Szegedi-Hallgató, E., Janacsek, K., Vékony, T., Tasi, L. A., Kerepes, L., Hompoth, E. A., ... Nemeth, D. (2017). Explicit instructions and consolidation promote rewiring of automatic behaviors in the human mind. Scientific Reports, 7(1), 4365. https://doi.org/10.1038/s41598-017-04500-3

Takács, Á., Kóbor, A., Chezan, J., Éltető, N., Tárnok, Z., Nemeth, D., ... Janacsek, K. (2018). Is procedural memory enhanced in Tourette syndrome? Evidence from a sequence learning task. Cortex, 100, 84-94. https://doi.org/10.1016/j.cortex.2017.08.037

Takács, Á., Shilon, Y., Janacsek, K., Kóbor, A., Tremblay, A., Németh, D., \& Ullman, M. T. (2017). Procedural learning in Tourette syndrome, ADHD, and comorbid TouretteADHD: Evidence from a probabilistic sequence learning task. Brain and Cognition, 117, 33-40. https://doi.org/10.1016/j.bandc.2017.06.009

Thomas, K. M., Hunt, R. H., Vizueta, N., Sommer, T., Durston, S., Yang, Y., \& Worden, M. S. (2004). Evidence of developmental differences in implicit sequence learning: An fMRI study of children and adults. Journal of Cognitive Neuroscience, 16(8), 13391351. https://doi.org/10.1162/0898929042304688 
Thut, G., \& Miniussi, C. (2009). New insights into rhythmic brain activity from TMS-EEG studies. Trends in Cognitive Sciences, 13(4), 182-189. https://doi.org/10.1016/j.tics.2009.01.004

Thut, G., \& Pascual-Leone, A. (2010). A review of combined TMS-EEG studies to characterize lasting effects of repetitive TMS and assess their usefulness in cognitive and clinical neuroscience. Brain Topography, 22(4), 219-232. https://doi.org/10.1007/s10548-009-0115-4

Trivedi, J. (2006). Cognitive deficits in psychiatric disorders: Current status. Indian Journal of Psychiatry, 48(1), 10. https://doi.org/10.4103/0019-5545.31613

Tse, N. Y., Goldsworthy, M. R., Ridding, M. C., Coxon, J. P., Fitzgerald, P. B., Fornito, A., \& Rogasch, N. C. (2018). The effect of stimulation interval on plasticity following repeated blocks of intermittent theta burst stimulation. Scientific Reports, 8(1), 8526. https://doi.org/10.1038/s41598-018-26791-w

Tupak, S. V., Dresler, T., Badewien, M., Hahn, T., Ernst, L. H., Herrmann, M. J., ... Fallgatter, A. J. (2013). Inhibitory transcranial magnetic theta burst stimulation attenuates prefrontal cortex oxygenation. Human Brain Mapping, 34(1), 150-157. https://doi.org/10.1002/hbm.21421

Turk-Browne, N. B., Scholl, B. J., Johnson, M. K., \& Chun, M. M. (2010). Implicit perceptual anticipation triggered by statistical learning. Journal of Neuroscience, 30(33), 11177-11187. https://doi.org/10.1523/jneurosci.0858-10.2010

Unoka, Z., Vizin, G., Bjelik, A., Radics, D., Nemeth, D., \& Janacsek, K. (2017). Intact implicit statistical learning in borderline personality disorder. Psychiatry Research, 255, 373-381. https://doi.org/10.1016/j.psychres.2017.06.072

Vallence, A. M., Goldsworthy, M. R., Hodyl, N. A., Semmler, J. G., Pitcher, J. B., \& Ridding, M. C. (2015). Inter- and intra-subject variability of motor cortex plasticity following continuous theta-burst stimulation. Neuroscience, 304, 266-278. https://doi.org/10.1016/j.neuroscience.2015.07.043

Vékony, T., Török, L., Pedraza, F., Schipper, K., Plèche, C., Tóth, L., ... Nemeth, D. (2019). Retrieval of a well-established skill is resistant to distraction: evidence from an implicit 
probabilistic sequence learning task. BioRxiv, 849729. https://doi.org/10.1101/849729

Viejo-Sobera, R., Redolar-Ripoll, D., Boixadós, M., Palaus, M., Valero-Cabré, A., \& Marron, E. M. (2017). Impact of prefrontal theta burst stimulation on clinical neuropsychological tasks. Frontiers in Neuroscience, 11, 462. https://doi.org/10.3389/fnins.2017.00462

Virag, M., Janacsek, K., Horvath, A., Bujdoso, Z., Fabo, D., \& Nemeth, D. (2015).

Competition between frontal lobe functions and implicit sequence learning: evidence from the long-term effects of alcohol. Experimental Brain Research, 233(7), 2081-2089. https://doi.org/10.1007/s00221-015-4279-8

Wager, T. D., \& Smith, E. E. (2003). Neuroimaging studies of working memory: a metaanalysis. Cognitive, Affective and Behavioral Neuroscience, 3(4), 255-274. https://doi.org/10.3758/CABN.3.4.255

Weigand, A., Grimm, S., Astalosch, A., Guo, J. S., Briesemeister, B. B., Lisanby, S. H., ... Bajbouj, M. (2013). Lateralized effects of prefrontal repetitive transcranial magnetic stimulation on emotional working memory. Experimental Brain Research, 227(1), 4352. https://doi.org/10.1007/s00221-013-3483-7

Weigand, A., Richtermeier, A., Feeser, M., Guo, J. S., Briesemeister, B. B., Grimm, S., \& Bajbouj, M. (2013). State-dependent effects of prefrontal repetitive transcranial magnetic stimulation on emotional working memory. Brain Stimulation, 6(6), 905-912. https://doi.org/10.1016/j.brs.2013.06.004

Weintraub, S., Wicklund, A. H., \& Salmon, D. P. (2012). The neuropsychological profile of Alzheimer disease. Cold Spring Harbor Perspectives in Medicine, 2(4), a006171. https://doi.org/10.1101/cshperspect.a006171

Yin, H. H., \& Knowlton, B. J. (2006). The role of the basal ganglia in habit formation. Nature Reviews Neuroscience, 7(6), 464-476. https://doi.org/10.1038/nrn1919 


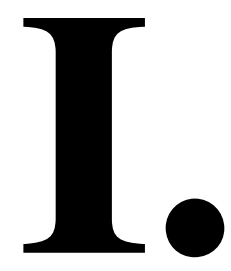




\section{SCIENTIFIC REP}

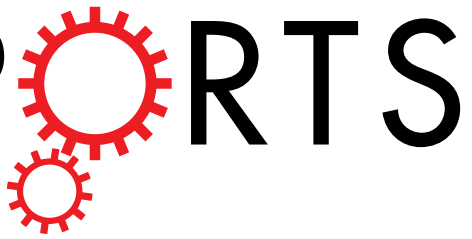
over the dorsolateral prefrontal cortex inhibits improvement on a

Received: 4 January 2018

Accepted: 24 September 2018

Published online: 04 October 2018

\section{working memory task}

Teodóra Vékony ${ }^{1}$, Viola Luca Németh ${ }^{1}$, Adrienn Holczer ${ }^{1}$, Krisztián Kocsis $^{1}$, Zsigmond Tamás Kincses ${ }^{1}$, László Vécsei ${ }^{1,2}$ \& Anita Must ${ }^{2,3}$

Theta-burst stimulation (TBS) over the dorsolateral prefrontal cortex (DLPFC) may be more effective for modulating cortical excitability compared to standard repetitive transcranial magnetic stimulation. However, the impact of intermittent (iTBS) and continuous TBS (cTBS) on working memory (WM) is poorly studied. The aim of our study was to compare the effects of iTBS and CTBS on WM over the left and right DLPFC. iTBS, CTBS or sham stimulation was administered over the right and left hemisphere of fifty-one healthy human subjects. WM was assessed before and after TBS using the 1-back, 2-back, and 3-back tasks. We found classical practice effects in the ITBS and the sham group: WM performance improved following stimulation as measured by the discriminability index. However, this effect could not be observed in the CTBS group. We did not find any hemisphere-dependent effects, suggesting that the practice effect is not lateralized, and TBS affects WM performance in a comparable manner if administered either over the left or the right hemisphere. We propose that our findings represent a useful addition to the literature of TBS-induced effects on WM. Moreover, these results indicate the possibility of clarifying processes underlying WM performance changes by using non-invasive brain stimulation.

Non-invasive brain stimulation has become a highly prosperous field of cognitive research in the last few decades. One of the available methods is repetitive transcranial magnetic stimulation (rTMS), that involve the application of rapid, brief magnetic fields to the scalp. These magnetic fields induce a current in the underlying tissues, leading to the depolarization of neurons in the targeted brain area ${ }^{1}$. Administering high-frequency rTMS (above $5 \mathrm{~Hz}$ ) over the dorsolateral prefrontal cortex (DLPFC) has been shown to improve working memory (WM). In contrast, applying low-frequency $(\leq 1 \mathrm{~Hz})$ rTMS results in performance disruption ${ }^{2-4}$. A relatively new and seemingly more effective alternative to rTMS is theta-burst stimulation (TBS), which uses gamma frequency trains applied at theta rhythm ${ }^{5}$. The pattern of TBS was designed to mimic theta-gamma coupling, i.e. the modulation of gamma power by theta phase ${ }^{6}$. This type of cross-frequency coupling serves as a tool for realizing effective coordination between several cortical areas during cognitive tasks ${ }^{7-9}$. Thus, it might be capable of inducing behavioral effects as theta-gamma coupling is involved in WM and long-term memory processes ${ }^{8,10-12}$. Two main type of TBS can be distinguished: the intermittent TBS (iTBS) is described as facilitating ${ }^{13}$, while the continuous TBS (cTBS) is expected to be suppressive on cortical excitability ${ }^{14}$. However, in the motor domain, TBS after-effects have been found to be highly variable across subjects ${ }^{15-18}$. Despite the advantages of TBS (its specific pattern, shorter stimulation period and longer-lasting effects, at least in the motor domain $)^{5,19}$, to date, only a few studies investigated the impact of TBS on $\mathrm{WM}^{20}$. Here we present a study comparing iTBS- and cTBS-induced effects on WM both over the right and left DLPFC.

Previous studies have found different effects of cTBS and iTBS on several cognitive domains. Interestingly, the distinct protocols did not consistently lead to opposite behavioral outcomes ${ }^{20,21}$. Three recent studies have investigated the effect of iTBS administration over the left DLPFC. Increased theta weighted phase-lag index between

${ }^{1}$ Department of Neurology, University of Szeged, Szeged, Hungary. ${ }^{2}$ MTA-SZTE Neuroscience Research Group, Szeged, Hungary. ${ }^{3}$ Institute of Psychology, University of Szeged, Szeged, Hungary. Correspondence and requests for materials should be addressed toT.V. (email: vekony.teodora@med.u-szeged.hu) or A.M. (email: must.anita@ med.u-szeged.hu) 
frontoparietal regions and increased parietal gamma power has been found following iTBS along with behavioral improvement in the n-back WM task ${ }^{22}$. Enhanced amplitudes of TMS-evoked event-related potentials ${ }^{23}$, as well as increased theta and gamma power, have also been detected by Chung et al. ${ }^{24}$. However, in these studies, consistent behavioral improvement could not be revealed ${ }^{23,24}$. On the other hand, cTBS over the DLPFC has been reported to impair $\mathrm{WM}^{25,26}$ and also to decrease theta power ${ }^{27}$. Given these inconsistencies, a systematic investigation of cTBS- and iTBS-related impact on WM and direct comparisons of the effect of the two stimulation protocols with the same stimulation parameters on WM are needed.

A recent study has compared changes in performance on several neurocognitive tests, both following iTBS and cTBS. WM performance decreased following both active stimulation protocols considering reaction times, as reflected by a diminished practice effect. However, only cTBS had a disruptive effect on task accuracy ${ }^{21}$. This suggests that cTBS does not (only) disrupt WM itself but (also) the capacity to improve in a WM task. Although practice effects have been considered to be a confounding factor in cognitive measurements, practice-related changes - as a tool to measure cognitive plasticity ${ }^{28}$ - also yield useful information about the underlying mechanism of cognitive performance ${ }^{29,30}$. It is currently not known whether the mechanism behind the practice-related improvement (i.e. the ability to learn task-specific skills) in a WM task can be interfered by a single session of non-invasive stimulation. This question might also be relevant for the interpretation of TBS effects on brain plasticity, which is a key mechanism for learning and memory ${ }^{16,18}$.

The above-mentioned TBS-studies have applied stimulation over the left DLPFC only. Thus, effects of TBS administration over the right hemisphere also remain an open question. A recent study has found that left but not right cTBS induces bilateral blood oxygenation changes in the prefrontal area ${ }^{31}$, and dopamine release is enhanced only following left DLPFC stimulation ${ }^{32}$. From the behavioral perspective, a direct comparison of TBS effects on WM over the left and right DLPFC is lacking in the current literature. Traditional TMS studies have found that verbal WM appears to be modified by left but not right DLPFC stimulation ${ }^{33,34}$. Others have reported rTMS to effectively modulate WM both over the left and the right DLPFC 335 -38. However, in some cases, the effects of rTMS over the right DLPFC have only been prominent for WM tasks containing spatial ${ }^{39,40}$ or negatively valenced stimuli ${ }^{41,42}$. Thus, clarifying whether DLPFC stimulation over the left and the right hemisphere is associated with verbal WM changes is warranted.

The above-mentioned gaps in the literature have raised the following questions for our study: (1) is iTBS improving and cTBS worsening WM performance, (2) are left and right DLPFC stimulation leading to different consequences considering their distinct effect on WM performance? Therefore, we administered iTBS, cTBS or sham stimulation over the left and right DLPFC of healthy participants. The n-back task, a well-established approach for WM assessment, has been administered twice: once before and once after the stimulation. Hoy et al. found behavioral improvement on WM tasks, as well as increased fronto-parietal theta synchronization and parietal gamma band power following iTBS ${ }^{22}$. Therefore, we expected an increase in performance on the n-back task following iTBS administration. Opposite behavioral and electrophysiological effects using cTBS have been reported by Schicktanz et al. ${ }^{25}$ and Chung et al. ${ }^{24}$, respectively. Thus, we hypothesized that cTBS would decrease WM performance. Our third hypothesis was that the effects related to TBS would not be equal over the two hemispheres. Based on previous findings, we expect more pronounced results following left DLPFC stimulation compared to right DLPFC stimulation ${ }^{31,33,34,43}$.

\section{Results}

Overview. A total of fifty-one participants completed the study. Subjects received iTBS, cTBS or sham stimulation over the DLPFC. At two separate occasions, the left and right hemisphere were stimulated, respectively. The n-back working memory task (1-back, 2-back, and 3-back) was completed by the participants both prior to and following the stimulation. Performance was assessed by reaction times and $\mathrm{d}^{\prime}$ scores.

Pre-stimulation RTs and d' scores. We did not find pre-stimulation differences between groups regarding both median RTs and d' scores. This is supported by non-significant GROUP main effects and the lack of interactions including GROUP (all p > 0.05) (for details, see Supplementary Tables S1 and S2). These results indicate an equal pre-stimulation performance of the three groups for all administered levels of the n-back task and for each condition.

Median RTs. The mixed ANOVA for median RTs revealed a significant main effect of $\mathrm{TIME}\left(\mathrm{F}_{(1,48)}=8.763\right.$, $\left.\mathrm{p}=0.005, \mathrm{n}_{\mathrm{p}}{ }^{2}=0.154\right)$. Post-hoc tests showed slightly shorter overall median RTs following stimulation (mean scores: pre-stimulation $0.618 \mathrm{~s} \pm 0.012 \mathrm{SE}$; post-stimulation $0.600 \mathrm{~s} \pm 0.011 \mathrm{SE}$ ). An additional main effect of LOAD was found $\left(\mathrm{F}_{(2,96)}=115.233, \mathrm{p}<0.001, \mathrm{n}_{\mathrm{p}}{ }^{2}=0.706\right)$, indicating increasing RTs with higher cognitive demand (mean scores: $0.499 \mathrm{~s} \pm 0.009$ SE for 1-back; $0.609 \mathrm{~s} \pm 0.014$ SE for 2-back; $0.719 \mathrm{~s} \pm 0.017$ SE for 3-back). The interaction of the two main effects was also found to be significant $\left(\mathrm{F}_{(1,96)}=4.947, \mathrm{p}=0.009, \mathrm{n}_{\mathrm{p}}{ }^{2}=0.093\right)$. Post-hoc comparison revealed that the difference between the two time points was detectable for the 2-back $\left(\mathrm{F}_{(1,48)}=9.137, \mathrm{p}=0.004, \mathrm{n}_{\mathrm{p}}{ }^{2}=0.160\right)$ and 3-back level $\left(\mathrm{F}_{(1,48)}=6.519 \mathrm{p}=0.014, \mathrm{n}_{\mathrm{p}}{ }^{2}=0.120\right)$, but not for 1-back $\left(\mathrm{F}_{(1,48)}=0.591, \mathrm{p}=0.446, \mathrm{n}_{\mathrm{p}}{ }^{2}=0.012, \mathrm{BF}_{10}=0.200\right)$. The above interaction was not modified by GROUP $\left(\mathrm{F}_{(4,96)}=1.358, \mathrm{p}=0.254, \mathrm{n}_{\mathrm{p}}{ }^{2}=0.054\right)$, or by $\operatorname{SIDE}\left(\mathrm{F}_{(1.689,81.095)}=0.314, \mathrm{p}=0.694, \mathrm{n}_{\mathrm{p}}{ }^{2}=0.007\right)$. To clarify whether there is truly no difference related to stimulation type, or our data are just not sensitive enough to detect the difference, we calculated Bayes-factor excluding $\left(\mathrm{H}_{0}\right)$ and including $\left(\mathrm{H}_{1}\right)$ the effect of GROUP. The Bayes-factor model indicated a strong evidence for $\mathrm{H}_{0}\left(\mathrm{BF}_{10}=0.050\right)$. We applied the similar algorithm for SIDE, indicating strong evidence in favor of $\mathrm{H}_{0}\left(\mathrm{BF}_{10}=0.064\right)$.

d' scores. The ANOVA of $\mathrm{d}^{\prime}$ scores revealed a significant main effect of $\operatorname{LOAD}\left(\mathrm{F}_{(1,48)}=344.822, \mathrm{p}<0.001\right.$, $\left.\mathrm{n}_{\mathrm{p}}{ }^{2}=0.878\right)$, as well as TIME $\left(\mathrm{F}_{(1,48)}=30.297, \mathrm{p}<0.001, \mathrm{n}_{\mathrm{p}}{ }^{2}=0.387\right)$. Post-hoc tests showed better performance 


\section{A) LEFT DLPFC}

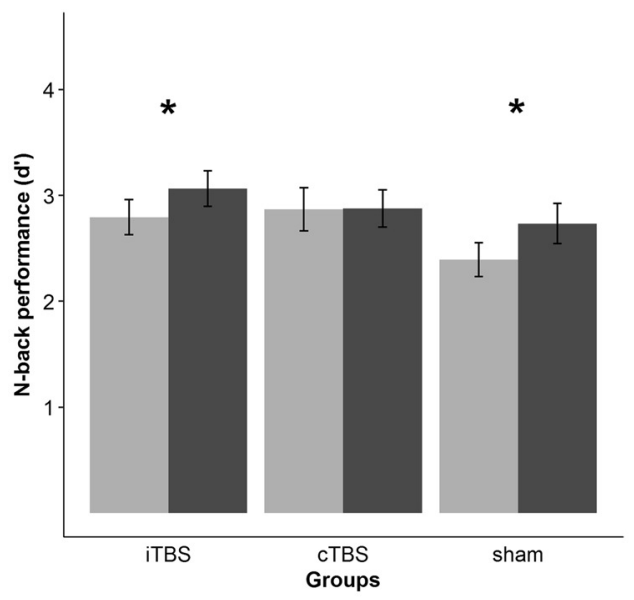

B) RIGHT DLPFC

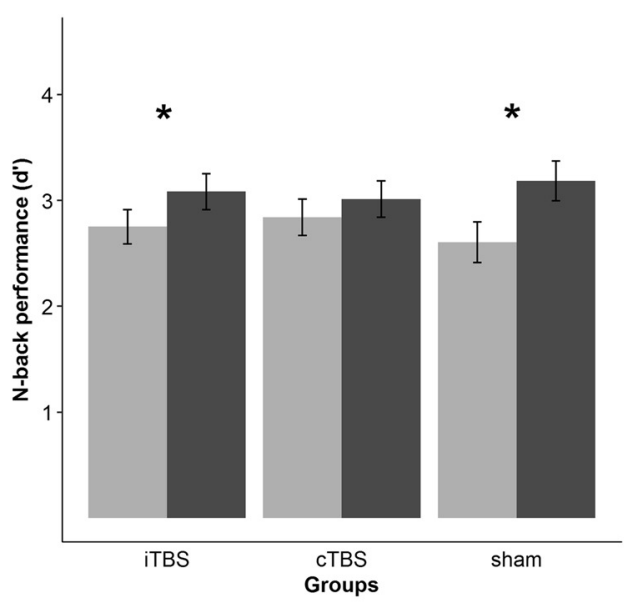

Figure 1. Pre- and post-stimulation n-back performance ( $\mathrm{d}^{\prime}$ scores on 2-back and 3-back) when stimulating over the left (A) and right (B) DLPFC. Error bars indicate the standard error of mean. $\mathrm{d}^{\prime}$ scores improved in the iTBS and sham, but not in the cTBS group following stimulation.

on the 2-back compared to 3-back condition, indicating a higher cognitive demand for the 3-back condition (mean scores: $3.575 \pm 0.065$ SE for 2-back; $2.125 \pm 0.088$ SE for 3-back). As for the main effect of TIME, increased performance was found following stimulation, signaling a potential practice effect between the two time points (mean scores: $2.708 \pm 0.072 \mathrm{SE}$ for pre-stimulation; $2.992 \pm 0.071 \mathrm{SE}$ for post-stimulation). Notably, a significant TIME $\times$ GROUP interaction was detected $\left(\mathrm{F}_{(2,48)}=4.252, \mathrm{p}=0.02, \mathrm{n}_{\mathrm{p}}{ }^{2}=0.151\right)$. To further analyze the source of this interaction, pairwise comparison with estimated marginal means was applied. We found a difference between the two time points in the iTBS $\left(\mathrm{F}_{(1,48)}=12.095, \mathrm{p}=0.001, \mathrm{n}_{\mathrm{p}}{ }^{2}=0.201\right)$ and the sham group $\left(\mathrm{F}_{(1,47)}=25.113\right.$, $\left.\mathrm{p}<0.001, \mathrm{n}_{\mathrm{p}}{ }^{2}=0.343\right)$, but this did not apply for the cTBS group $\left(\mathrm{F}_{(1,48)}=0.999, \mathrm{p}=0.323, \mathrm{n}_{\mathrm{p}}{ }^{2}=0.02\right.$, $\mathrm{BF}_{10}=0.398$ ) (Fig. 1). The interaction above was not modified by $\operatorname{SIDE}\left(\mathrm{F}_{(1,48)}=0.198, \mathrm{p}=0.821, \mathrm{n}_{\mathrm{p}}{ }^{2}=0.008\right)$. To support the finding that SIDE as a factor has no critical effect on the detected interaction, Bayes-factors were calculated excluding $\left(\mathrm{H}_{0}\right)$ and including $\left(\mathrm{H}_{1}\right)$ for the effect of SIDE. In this model, Bayes-factor indicated strong evidence in favor of $\mathrm{H}_{0}\left(\mathrm{BF}_{10}=0.096\right)$.

\section{Discussion}

In the current study, we aimed to investigate the effects of iTBS and cTBS delivered over the right and left DLPFC on two repeated measurements of WM performance. The n-back task has been administered before and after stimulation. The expected practice effect (i.e. general improvement due to repeated task completion ${ }^{44}$ ) occurred in the iTBS and sham stimulation group. This was reflected by the discriminability index. However, cTBS eliminated the practice effect and thus reducing WM performance. Considering RTs, practice-related effects occurred independently of stimulation type. Moreover, we detected a similar impact of TBS on both hemispheres. Bayesian statistics further support our findings obtained with more classical statistical approaches.

TBS has been shown to modulate brain activity by mimicking theta-gamma coupling ${ }^{5}$ which is a key feature for realizing coordination during cognitive tasks ${ }^{7-9}$. Thus, the effect of TBS is expected to modulate WM based on the theta-gamma neural code ${ }^{11}$. Previous studies have found a disruptive effect of cTBS on WM measured by behavioral methods ${ }^{25,26}$. Therefore, our results suggesting reduced WM performance (i.e. lack of improvement) following cTBS are in line with previous findings. We might argue that cTBS affected WM performance by causing long-term depression-like effects or by impairing the dopaminergic transmission in networks involving the DLPFC $^{5,32,45}$. Besides that, the modulation of theta-gamma oscillations could also play a role in the cTBS-related WM effects. Studies have found cTBS to increase the power of theta-gamma frequency oscillations over the DLPFC, whereas iTBS has been reported to increase it ${ }^{22,23,27}$.

Contrary to our hypothesis, a lack of iTBS effects was found in the current study. iTBS has been reported to enhance task-related theta-gamma synchronization between frontal and parietal areas ${ }^{22,24}$, and subtle effects of iTBS on WM have also been shown ${ }^{22}$. However, some recent studies have also failed to find behavioral enhancement after iTBS accompanied by oscillatory changes ${ }^{23,24}$. Behavioral modulations are difficult to show after a single session of stimulation of healthy individuals ${ }^{2}$, and the behavioral consequences of cTBS on cognition might be more stable than iTBS effects ${ }^{46}$. This assumption could partially explain why we only detected cTBS-related changes on the behavioral level. Studies show that iTBS is more likely to alter electrophysiological markers (i.e. to cause differences in the features of event-related potentials, to change the degree of theta-gamma coupling or power in the crucial frequency ranges) than to cause behavioral changes ${ }^{23,24}$. Thus, iTBS might have had an effect on the neural level, which was not manifested on the behavioral level. Nevertheless, as WM-related electrophysiological after-effects of cTBS are not revealed yet, further studies should be conducted to clarify the underlying mechanism of the cTBS-induced behavioral changes, also compared to iTBS. 
We might also speculate that the revealed disruptive effect of cTBS was not necessarily specific to WM itself, but rather to the consolidation of task-specific skills. Therefore, practice effects might also be interpreted as task-related knowledge which would help participants to improve their performance on the second administration. This might be supported by the lack of practice effect reported in neurocognitive disorders ${ }^{47,48}$, which are characterized by deficits in memory consolidation and acquiring new information ${ }^{49}$. Considering that memory processes may be modified by TBS ${ }^{50,51}$, and theta frequency oscillations are associated with memory consolidation processes ${ }^{12}$, it might be possible that prefrontal stimulation affected the consolidation of task-related skills. Future studies assessing whether consolidation may also play a role in TBS-related WM performance changes, seem warranted.

We did not find practice effects to occur in the 1-back condition. This supports the assumption that the changes detected here are WM-dependent to some extent and not solely associated with a more general task-related skill learning ability. Practice-related effects have been previously described to be independent of task difficulty ${ }^{52}$. However, different levels of performance modulation (or the lack of practice-related behavioral changes) following sham TBS have been found on several neuropsychological tasks ${ }^{21}$. A recent study has also revealed that the practice effect consists of various sub-processes, which are differently affected by the repeated completion of the task ${ }^{53}$. In the current study, processing speed was not modulated by TBS, thus supporting the notion that the applied stimulation affected only sub-processes defining WM performance. Nevertheless, the question if the measured effects originate from alterations in task-independent model-building processes or from solely WM-related changes remains in the focus of interest for future research. This could be addressed by measuring TBS-induced changes on the practice effect using other executive function tests with less WM demand (for example, Attention Network Task ${ }^{54}$ ). Additionally, the longitudinal assessment of practice effects (i.e. through multiple testing sessions) could reveal its dynamics and potential specificities. To sum up, we suggest that the disruption of practice effects related to cTBS might serve as a helpful concept to explain the inhibitory influence of cTBS on cognitive performance, whereas iTBS appears to be less robust than cTBS in this aspect.

Previous rTMS findings have reported bilateral contribution or even right DLPFC superiority for WM tasks using different cognitive targets $3,35-37,39,40,42$. In contrast, an early TMS study has suggested the left DLPFC stimulation leads to verbal WM performance modulation ${ }^{33}$. Interestingly, neuroimaging data have also suggested left DLPFC superiority for $\mathrm{WM}^{43}$. The n-back task used here was verbally featured, with no spatial or emotional aspects, which could partially explain the equal effects of TBS over the left and right hemisphere. We might argue that the left and right DLPFC play a similar role in the tested WM-related learning ability. Another possible explanation might be that disrupting activity in either hemisphere provokes an imbalance between the hemispheres that results in impaired performance ${ }^{55}$. This would also mean that enhancing activity using iTBS or high-frequency rTMS on one hemisphere could cause an imbalance that ultimately reduces performance. In studies using facilitatory TMS-methods either increase or a lack of change in performance has been detected ${ }^{2,46}$. This makes the hemispheric imbalance explanation less plausible. However, future studies specifically addressing this issue are required. Our current results further support the notion that WM-related processes can be equally modulated by TBS over either hemisphere in healthy individuals.

The stimulation intensity is crucial for the interpretation of our results considering that it differed remarkably from the intensity levels applied by previous studies testing the effects of TBS on WM. Nevertheless, cognitive changes have been described previously with comparable or even lower intensities ${ }^{56-58}$. The average MT of our participants was approximately $60 \%$ of the MSO. Thus, the stimulation intensity level was at nearly $50 \%$ of MT, compared to $80 \%$ of MT typically used in similar studies ${ }^{22,25}$. Consequently, we might speculate that cTBS exerts its effect on WM at low intensity, whereas iTBS might require higher intensities to have a more prominent or longer-lasting effect on WM performance. Additionally, iTBS and cTBS are characterized by distinct electrophysiological parameters, with the ideal stimulation intensity also differing if applied over motor cortical areas ${ }^{5}$. Similar to our findings, a diminished practice effect on n-back performance following cTBS has been previously found when comparing it to iTBS and sham stimulation ${ }^{21}$. The stimulation intensity applied by Viejo-Sobera et al. ${ }^{21}$ was $80 \%$ of the active motor threshold. This value is lower compared to motor threshold measured without voluntary contraction, typically by $5-20 \%$ of the $\mathrm{MSO}^{59}$. Thus, the level of intensity was comparable to our administration but lower than in studies reporting an increase in WM performance following iTBS ${ }^{22}$. Additionally, a recent study aimed to identify the ideal stimulation intensity for iTBS to modulate WM. This revealed an inverse U-shaped pattern of the different stimulation intensity effects, with nearly $75 \%$ of resting MT resulting in the largest neurophysiological changes. Nevertheless, $50 \%$ of resting MT also influenced neurophysiological functioning - but not at the behavioral level ${ }^{24}$. Exact data on the ideally recommended stimulation intensity of cTBS to affect WM is not yet available, therefore, future studies should address this issue. We strongly believe that our current results shed further light on potentially distinct but optimal stimulation intensities of different TBS protocols. This is supported by the disruptive effect of cTBS at the applied intensity, whereas iTBS remained ineffective at the same intensity.

In the present study, we found cTBS to have an inhibitory influence on the practice effect during the n-back task, while iTBS did not exert this fundamental effect. Our findings suggest that (1) the two distinct stimulation protocols presumably exert different effects on measured cognitive abilities, and (2) stimulation of the left or right DLFPC might have equivalent effects on WM at the behavioral level. Furthermore, in the light of previously reported neurophysiological changes related to WM following TBS administration ${ }^{22,24,25}$, future studies should examine post-TBS neuronal responses if stimulating both hemispheres. The role of practice underlying WM performance alterations and its interaction with TBS should be systematically explored in the future. The current results also emphasize the importance of investigating effects of low-intensity TBS on cognitive function and the potential differences of the ideal stimulation intensity for iTBS and cTBS to modulate WM. Above this, the assumption that iTBS and cTBS are defined by distinct ideal stimulation intensity levels could well influence the design of TBS-based treatment protocols. We strongly believe that the current findings represent a useful addition 


\begin{tabular}{|l|l|l|l|}
\hline & cTBS & iTBS & sham \\
\hline Gender $(\mathrm{f} / \mathrm{m})$ & $6 / 11$ & $8 / 10$ & $12 / 4$ \\
\hline Mean age & $24.23 \pm 2.81$ S.D. & $25.27 \pm 2.65$ S.D. & $21.31 \pm 2.3$ S.D. \\
\hline Education (undergradute/postgraduate) & $7 / 10$ & $8 / 10$ & $10 / 6$ \\
\hline Handedness (r/l) & $16 / 1$ & $17 / 1$ & $14 / 2$ \\
\hline
\end{tabular}

Table 1. Demographical data.

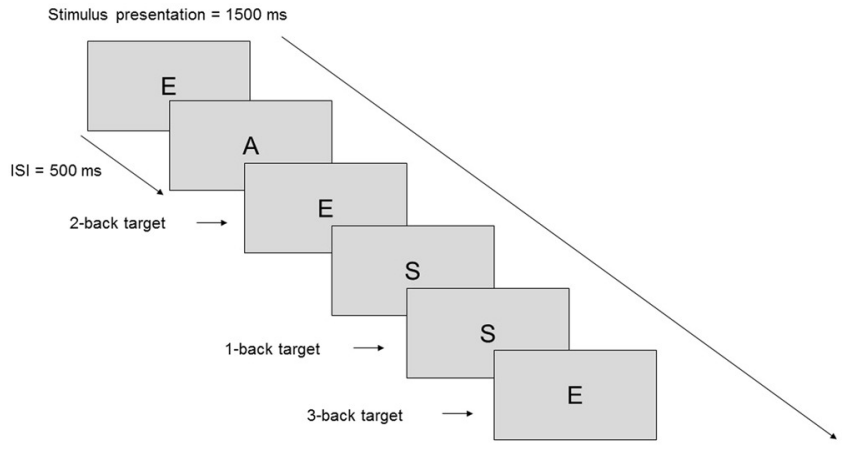

Figure 2. Illustration of the n-back task. Level 1-back, 2-back, and 3-back were tested separately.

to the process of developing effective ways to reveal the influence of iTBS and cTBS on WM and to clarify the sub-processes behind WM changes by using non-invasive brain stimulation.

\section{Methods}

Participants. The selected sample size was based on an a priori sample size estimation. We calculated the required sample size with a medium estimated effect size, as TBS-induced effect sizes were found to be larger than in rTMS studies ${ }^{5,22}$. To find group differences between the three groups and within two repeated measurements with a power of 0.85 , and assuming a medium effect size of 0.5 , the required sample size was 48 . Fifty-two healthy volunteers were recruited to participate in the study (see Table 1 for demographic data). None of the subjects had a history of any psychiatric or neurological disorder at the time of the participation. All participants had normal or corrected-to-normal vision. Participants were randomly assigned to one of the three groups and were naïve to the stimulation type. Informed consent was signed by all participants prior to the first session, and none of them withdrew from the experiment because of TBS discomfort. We had to exclude one participant due to administration failure in the first session. Thus, final analysis was carried out including fifty-one participants. The study was conducted in accordance with the Declaration of Helsinki, and the experimental protocol was approved by the Regional Scientific and Research Ethics Committee, Albert Szent-Györgyi Health Center, University of Szeged (Ref. no.: 165/2014).

Experimental design. The examination was performed in two separate sessions. At least two weeks of washout period was kept between the two occasions. During both sessions, all participants completed three levels of the $n$-back task before and after stimulation. Either the right or the left DLPFC was stimulated during the first examination, with the other hemisphere on the second occasion, respectively. The order of stimulation side was counterbalanced across participants. Three groups were formed: eighteen out of the fifty-one participants were given iTBS, seventeen of them received cTBS, and sixteen participants were assigned to the sham stimulation group. The experimental protocol was identical in each group, except for the type of stimulation.

N-back task. The 1-back, 2-back, and 3-back version of the n-back task was administered consecutively ${ }^{60}$ using PsychoPy (version: v1.82.01) ${ }^{61}$. During the n-back tasks, random capital letter stimuli (A, C, E, I, K, L, S, O, $\mathrm{R}, \mathrm{T}, \mathrm{U}$ ) were presented serially on the screen for $1500 \mathrm{~ms}$ with an interstimulus interval of $500 \mathrm{~ms}$. Participants had to respond by pressing the space bar if the letter on the screen was the same as the letter presented one (1-back task), two (2-back task) or three (3-back task) trials earlier (Fig. 2). A total of 100 trials was completed at each level meaning a total of 300 trials per measurement. The frequency of target stimuli was set at $20 \%$ of all presented stimuli. The total duration of the tasks was about 15 minutes. We assumed that post-stimulation effects lasted during the entire examination, based on previous studies using TBS to modulate cognitive performance ${ }^{22,25}$. Reaction times (RT), number of hits, correct rejections, false alarms and misses were recorded.

Theta-burst stimulation protocol. TBS was generated by a Magstim Rapid ${ }^{2}$ stimulator with a D70² $70 \mathrm{~mm}$ figure-of-eight coil (The Magstim Company Ltd, Whitland, Wales, UK). Prior to the experiment, each participant in the iTBS and cTBS group went through an anatomical T1-weighted MRI-scan using a 1.5T GE Signa Excite HDxt scanner (Milwaukee, WI, USA): 3D IR-FSPGR: TR/TE/TI: 10.3/4.2/450 ms; flip angle: 15; ASSET: 2, FOC: $25 \times 25 \mathrm{~cm}$; matrix: $256 \times 256$; slice thickness: $1 \mathrm{~mm}$. A TMS Neuronavigator (Brain Innovation, Maastricht, the Netherlands) with ultrasound CMS20 Measuring System (Zebris GmbH, Tübingen, Germany) 


\begin{tabular}{|l|l|l|l|}
\hline & cTBS & iTBS & sham \\
\hline Mean rMT (\% of MSO) & $62.35 \pm 12.81$ S.D. & $59.72 \pm 13$ S.D. & $55.69 \pm 10.26$ S.D. \\
\hline Range (\% of MSO) & $34-78$ & $43-80$ & $36-70$ \\
\hline
\end{tabular}

Table 2. rMT details.

was used to localize the target position on the scalp of each participant. Prior to this, 3D-brain models were created using participants' MRI scans for accurate and individual targeting. Right and left DLPFC has been marked as the target area located on the 3D surface rendering of the brain, based on each participants' gyral morphology (the anterior third of the middle frontal gyrus, Brodmann 9/46). We positioned the center of the coil over the target area, tangentially to the skull, with the handle pointing backward. During sham stimulation, the coil was rotated $45^{\circ}$ away from the skull, with one wing of the coil being in contact with the scalp. Within the sham group, we administered the iTBS protocol for half of the participants, and cTBS pattern for the other half of the participants.

The cTBS and iTBS administration protocols in the current study was based on the original TBS stimulation pattern described by Huang et al. ${ }^{5}$. The cTBS pattern consisted of 3 pulses given at $50 \mathrm{~Hz}$ (gamma frequency) in every $200 \mathrm{~ms}$ (theta frequency intervals of $5 \mathrm{~Hz}$ ) for $40 \mathrm{~s}$. Thus, a total of 600 pulses of uninterrupted TBS was administered to each participant in the cTBS group. As for iTBS, a $2 \mathrm{~s}$ train was repeated every $10 \mathrm{~s}$ for $190 \mathrm{~s}$ in total. In one train, 3 pulses were given at $50 \mathrm{~Hz}$, which resulted in 600 pulses for each subject in the iTBS group. The stimulation intensity was kept at $30 \%$ of the maximal stimulator output (MSO) of the Magstim Rapid ${ }^{2}$ stimulator, due to limitations in the maximal TBS intensity of the stimulator. We chose to administer equal intensity for all participants. This was based on evidence claiming that the individual adaptation of TMS intensity to the measured motor threshold does not necessarily lead to more prominent effects ${ }^{62}$. To make sure that the potential effects are not due to differences in the motor thresholds, we measured visible motor threshold (MT) without voluntary contraction prior to the two TBS sessions, at a separate occasion. It was defined as the lowest stimulation intensity applied over the right primary motor cortex (M1) required to elicit visible contraction of the left abductor pollicis brevis muscle in 3 out of 5 probes. If $80 \%$ of the individual MT did not reach $30 \%$ of MSO so that the intensity does not exceed the $80 \%$ of MT, the applied intensity was reduced by $20 \%$ of the MT. This occurred in one case in the cTBS group ( $27 \%$ of MSO) and in one case in the sham group ( $29 \%$ of MSO). The mean rMTs and ranges are given in Table 2. There was no difference between groups in terms of the mean $\operatorname{rMT}\left(\mathrm{F}_{(2,48)}=1.325\right.$, $\left.\mathrm{p}=0.275, \mathrm{n}_{\mathrm{p}}^{2}=0.52, \mathrm{BF}_{10}=0.385\right)$.

Statistical analysis. For statistical analysis with the frequentist approach, IBM SPSS Statistics 24.0 software package was used. N-back performance was evaluated by two scores: median reaction times (RT) and discriminability index $\left(\mathrm{d}^{\prime}\right)$ used in the framework of signal detection theor $\mathrm{y}^{63}$. We chose to use median RTs over mean scores to avoid the confounding effect of invalid (i.e. extremely short or extremely long) RTs ${ }^{64}$. For calculating $\mathrm{d}^{\prime}$ scores, we distinguished four types of answers: hits (correctly identified targets), misses (incorrectly identified targets as non-targets), false alarms (incorrectly identified non-targets as targets), and correct rejections (correctly identified non-targets). The $\mathrm{d}^{\prime}$ value is a highly sensitive statistical index involving both the ability to maximize hits and the efficiency of minimizing false alarms. It is calculated from the standard deviation of the signal and the noise distribution, with higher scores representing more readily detected signals (therefore greater discriminability) ${ }^{65}$. $\mathrm{d}^{\prime}$ scores were calculated individually as:

$$
d^{\prime}=Z \text { (hit rate) }-Z \text { (false alarm rate) }
$$

We analyzed RTs by a $3 \times 2 \times 2 \times 2$ mixed ANOVA with the cognitive load (LOAD: 1. 1-back; 2. 2-back; 3 . 3-back), the time of administration (TIME: 1. pre-stimulation; 2. post-stimulation) and the side of the stimulation (SIDE: 1. right DLPFC; 2. left DLPFC) as within-subject factors, and stimulation type (GROUP: 1. iTBS; 2. cTBS; 3. sham) as between-subject factor. Considering that participants completed the 1-back task with an extremely high accuracy (99.53\% in total), $\mathrm{d}^{\prime}$ scores were not calculated for this task. Thus, $\mathrm{d}^{\prime}$ scores have been analyzed by a $2 \times 2 \times 2 \times 2$ mixed ANOVA with the same parameters as for the RTs, excluding the 1-back condition. Pairwise comparisons of estimated marginal means (with Bonferroni correction for multiple testing) was used for follow-up on the significant main effects and interactions. Prior to analyzing TBS-induced effects, an additional mixed ANOVA with LOAD and SIDE as within-subject factors, and with GROUP as a between-subject factor was carried out, to test whether the three groups differed in terms of their pre-stimulation performance (factor levels were the same as previously defined).

In addition to the more conventional frequentist statistical approach, we also calculated Bayes-factors (BF) for the underpinning of our non-significant, but relevant results. Although the classical null-hypothesis testing mainly relies on the $\mathrm{p}$-value, it is important to keep in mind that a non-significant result could mean at least two different things: (1) the null hypothesis is true; or (2) the collected data are not sensitive enough to distinguish between the null and an alternative hypothesis ${ }^{66}$. BF could be considered as the weight of evidence provided by the collected data, helping to differentiate between these two options ${ }^{67}$. Therefore, to compare the likelihood of our models favoring the $\mathrm{H}_{0}$ versus the $\mathrm{H}_{1}$ hypothesis, we performed Bayesian ANOVAs using JASP 0.8.03.01 ${ }^{68}$ carried out by default prior. Here we report $\mathrm{BF}_{10}$ values that should be interpreted as follows: $\mathrm{BF}_{10}$ values between 1 and 0.33 indicate anecdotal, values between 0.33 and 0.1 substantial, and values below 0.1 strong evidence for $\mathrm{H}_{0}$. Reversely, values between 1 and 3 indicate anecdotal, values between 3 and 10 substantial, and values above 10 strong evidence in favor of $\mathrm{H}_{1}{ }^{67}$. 


\section{References}

1. Hallett, M. Transcranial magnetic stimulation: a primer. Neuron 55, 187-199 (2007).

2. Brunoni, A. R. \& Vanderhasselt, M.-A. Working memory improvement with non-invasive brain stimulation of the dorsolateral prefrontal cortex: A systematic review and meta-analysis. Brain Cogn. 86, 1-9 (2014).

3. Mottaghy, F. M., Döring, T., Müller-Gärtner, H. W., Töpper, R. \& Krause, B. J. Bilateral parieto-frontal network for verbal working memory: An interference approach using repetitive transcranial magnetic stimulation (rTMS). Eur. J. Neurosci. 16, 1627-1632 (2002).

4. Lage, C., Wiles, K., Shergill, S. S. \& Tracy, D. K. A systematic review of the effects of low-frequency repetitive transcranial magnetic stimulation on cognition. J. Neural Transm. 123, 1479-1490 (2016).

5. Huang, Y. Z., Edwards, M. J., Rounis, E., Bhatia, K. P. \& Rothwell, J. C. Theta burst stimulation of the human motor cortex. Neuron 45, 201-206 (2005).

6. Lisman, J. \& Buzsáki, G. A neural coding scheme formed by the combined function of gamma and theta oscillations. Schizophr. Bull. 34, 974-980 (2008).

7. Niessing, J. et al. Neuroscience: Hemodynamic signals correlate tightly with synchronized gamma oscillations. Science (80-.). 309, 948-951 (2005).

8. Axmacher, N. et al. Cross-frequency coupling supports multi-item working memory in the human hippocampus. Proc. Natl. Acad. Sci. 107, 3228-3233 (2010).

9. Canolty, R. T. et al. High gamma power is phase-locked to theta oscillations in human neocortex. Science (80-.). 313, 1626-1628 (2006).

10. Lisman, J. Working memory: The importance of theta and gamma oscillations. Curr. Biol. 20, R490-R492 (2010).

11. Lisman, J. E. \& Jensen, O. The theta-gamma neural code. Neuron 77, 1002-1016 (2013).

12. Düzel, E., Penny, W. D. \& Burgess, N. Brain oscillations and memory. Curr. Opin. Neurobiol. 20, 245-257 (2010).

13. Di Lazzaro, V. et al. The physiological basis of the effects of intermittent theta burst stimulation of the human motor cortex. J. Physiol. 586, 3871-3879 (2008).

14. Di Lazzaro, V. et al. Theta-burst repetitive transcranial magnetic stimulation suppresses specific excitatory circuits in the human motor cortex. J. Physiol. 565, 945-50 (2005).

15. Hamada, M., Murase, N., Hasan, A., Balaratnam, M. \& Rothwell, J. C. The role of interneuron networks in driving human motor cortical plasticity. Cereb. Cortex 23, 1593-1605 (2013).

16. Vallence, A. M. et al. Inter- and intra-subject variability of motor cortex plasticity following continuous theta-burst stimulation. Neuroscience 304, 266-278 (2015).

17. Jannati, A., Block, G., Oberman, L. M., Rotenberg, A. \& Pascual-Leone, A. Interindividual variability in response to continuous theta-burst stimulation in healthy adults. Clin. Neurophysiol. 128, 2268-2278 (2017).

18. Tse, N. Y. et al. The effect of stimulation interval on plasticity following repeated blocks of intermittent theta burst stimulation. Sci. Rep. 8, 8526 (2018).

19. Suppa, A. et al. Ten years of theta burst stimulation in humans: established knowledge, unknowns and prospects. Brain Stimul. 9 , 323-335 (2016)

20. Demeter, E. Enhancing cognition with theta burst stimulation. Curr. Behav. Neurosci. Reports 3, 87-94 (2016).

21. Viejo-Sobera, R. et al. Impact of prefrontal theta burst stimulation on clinical neuropsychological tasks. Front. Neurosci. 11, 462 (2017).

22. Hoy, K. E. et al. Enhancement of working memory and task-related oscillatory activity following intermittent theta burst stimulation in healthy controls. Cereb. Cortex 1-11, https://doi.org/10.1093/cercor/bhv193 (2016).

23. Chung, S. W., Rogasch, N. C., Hoy, K. E. \& Fitzgerald, P. B. The effect of single and repeated prefrontal intermittent theta burst stimulation on cortical reactivity and working memory. Brain Stimul. 11, 566-574 (2018).

24. Chung, S. W. et al. Impact of different intensities of intermittent theta burst stimulation on the cortical properties during TMS-EEG and working memory performance. Hum. Brain Mapp. https://doi.org/10.1002/hbm.23882 (2017).

25. Schicktanz, N. et al. Continuous theta burst stimulation over the left dorsolateral prefrontal cortex decreases medium load working memory performance in healthy humans. PLoS One 10, e0120640 (2015).

26. Lee, T. G. \& D'Esposito, M. The dynamic nature of top-down signals originating from prefrontal cortex: a combined fMRI-TMS study. J. Neurosci. 32, 15458-15466 (2012).

27. Chung, S. W. et al. Demonstration of short-term plasticity in the dorsolateral prefrontal cortex with theta burst stimulation: A TMSEEG study. Clin. Neurophysiol. 128, 1117-1126 (2017).

28. Calero, M. D. \& Navarro, E. Relationship between plasticity, mild cognitive impairment and cognitive decline. Arch. Clin. Neuropsychol. 19, 653-660 (2004).

29. Duff, K. et al. Practice effects in the prediction of long-term cognitive outcome in three patient samples: A novel prognostic index. Arch. Clin. Neuropsychol. 22, 15-24 (2007).

30. Duff, K., Callister, C., Dennett, K. \& Tometich, D. Practice effects: A unique cognitive variable. Clin. Neuropsychol. 26, 1117-1127 (2012).

31. Tupak, S. V. et al. Inhibitory transcranial magnetic theta burst stimulation attenuates prefrontal cortex oxygenation. Hum. Brain Mapp. 34, 150-157 (2013).

32. Ko, J. H. et al. Theta burst stimulation-induced inhibition of dorsolateral prefrontal cortex reveals hemispheric asymmetry in striatal dopamine release during a set-shifting task - A TMS-[11C]raclopride PET study. Eur. J. Neurosci. 28, 2147-2155 (2008).

33. Mull, B. R. \& Seyal, M. Transcranial magnetic stimulation of left prefrontal cortex impairs working memory. Clin. Neurophysiol. 112, $1672-1675$ (2001).

34. Shields, J., Mock, J., Devier, D. \& Foundas, A. Unilateral repetitive transcranial magnetic stimulation differentially affects younger and older adults completing a verbal working memory task. J. Neurol. Sci. 384, 15-20 (2017).

35. Mottaghy, F. M. et al. Modulation of the neuronal circuitry subserving working memory in healthy human subjects by repetitive transcranial magnetic stimulation. Neurosci. Lett. 280, 167-170 (2000).

36. Preston, G., Anderson, E., Silva, C., Goldberg, T. \& Wassermann, E. M. Effects of $10 \mathrm{~Hz}$ rTMS on the neural efficiency of working memory. J. Cogn. Neurosci. 22, 447-456 (2010).

37. Hamidi, M., Tononi, G. \& Postle, B. R. Evaluating the role of prefrontal and parietal cortices in memory-guided response with repetitive transcranial magnetic stimulation. Neuropsychologia 47, 295-302 (2009).

38. Hulst, H. E. et al. RTMS affects working memory performance, brain activation and functional connectivity in patients with multiple sclerosis. J. Neurol. Neurosurg. Psychiatry 88, 386-394 (2017).

39. Sandrini, M., Rossini, P. M. \& Miniussi, C. Lateralized contribution of prefrontal cortex in controlling task-irrelevant information during verbal and spatial working memory tasks: rTMS evidence. Neuropsychologia 46, 2056-2063 (2008).

40. Fried, P. J., Rushmore, R. J., Moss, M. B., Valero-Cabré, A. \& Pascual-Leone, A. Causal evidence supporting functional dissociation of verbal and spatial working memory in the human dorsolateral prefrontal cortex. Eur. J. Neurosci. 39, 1973-1981 (2014).

41. Weigand, A. et al. State-dependent effects of prefrontal repetitive transcranial magnetic stimulation on emotional working memory. Brain Stimul. 6, 905-912 (2013). 
42. Weigand, A. et al. Lateralized effects of prefrontal repetitive transcranial magnetic stimulation on emotional working memory. Exp. Brain Res. 227, 43-52 (2013).

43. Wager, T. D. \& Smith, E. E. Neuroimaging studies of working memory: A meta-analysis. Cogn. Affect. Behav. Neurosci. 3, 255-274 (2003).

44. Calamia, M., Markon, K. \& Tranel, D. Scoring higher the second time around: Meta-analyses of practice effects in neuropsychological assessment. Clin. Neuropsychol. 26, 543-570 (2012).

45. Rossini, P. M. et al. Non-invasive electrical and magnetic stimulation of the brain, spinal cord, roots and peripheral nerves: Basic principles and procedures for routine clinical and research application: An updated report from an I.F.C.N. Committee. Clin. Neurophysiol. 126, 1071-1107 (2015).

46. Lowe, C. J., Manocchio, F., Safati, A. B. \& Hall, P. A. The effects of theta burst stimulation (TBS) targeting the prefrontal cortex on executive functioning: A systematic review and meta-analysis. Neuropsychologia 111, 344-359 (2018).

47. Duff, K. et al. Practice effects predict cognitive outcome in amnestic mild cognitive impairment. Am. J. Geriatr. Psychiatry 19, 932-939 (2011)

48. Ivnik, R. J. et al. Diagnostic accuracy of four approaches to interpreting neuropsychological test data. Neuropsychology 14, 163-177 (2000).

49. Weintraub, S., Wicklund, A. H. \& Salmon, D. P. The neuropsychological profile of Alzheimer disease. Cold Spring Harb. Perspect. Med. 2, a006171 (2012).

50. Demeter, E., Mirdamadi, J. L., Meehan, S. K. \& Taylor, S. F. Short theta burst stimulation to left frontal cortex prior to encoding enhances subsequent recognition memory. Cogn. Affect. Behav. Neurosci. 16, 724-735 (2016).

51. Blumenfeld, R. S., Lee, T. G. \& D’Esposito, M. The effects of lateral prefrontal transcranial magnetic stimulation on item memory encoding. Neuropsychologia 53, 197-202 (2014).

52. Reeve, C. L. \& Lam, H. The relation between practice effects, test-taker characteristics and degree of g-saturation. Int. J. Test. 7, 225-242 (2007).

53. Dutilh, G., Krypotos, A. M. \& Wagenmakers, E. J. Task-related versus stimulus-specific practice: A diffusion model account. Exp. Psychol. 58, 434-442 (2011).

54. Fan, J., McCandliss, B. D., Sommer, T., Raz, A. \& Posner, M. I. Testing the efficiency and independence of attentional networks. J. Cogn. Neurosci. https://doi.org/10.1162/089892902317361886 (2002).

55. Grimm, S. et al. Imbalance between left and right dorsolateral prefrontal cortex in major depression is linked to negative emotional judgment: an fMRI study in severe major depressive disorder. Biol. Psychiatry 63, 369-376 (2008).

56. Verbruggen, F., Aron, A. R., Stevens, M. A. \& Chambers, C. D. Theta burst stimulation dissociates attention and action updating in human inferior frontal cortex. Proc. Natl. Acad. Sci. 107, 13966-13971 (2010).

57. Dambacher, F. et al. The role of right prefrontal and medial cortex in response inhibition: interfering with action restraint and action cancellation using transcranial magnetic brain stimulation. J. Cogn. Neurosci. 26, 1775-1784 (2014).

58. Drummond, N. M., Cressman, E. K. \& Carlsen, A. N. Offline continuous theta burst stimulation over right inferior frontal gyrus and pre-supplementary motor area impairs inhibition during a go/no-go task. Neuropsychologia 99, 360-367 (2017).

59. Chen, R. et al. Intracortical inhibition and facilitation in different representations of the human motor cortex. J. Neurophysiol. 80, 2870-81 (1998)

60. Gevins, A. \& Cutillo, B. Spatiotemporal dynamics of component processes in human working memory. Electroencephalogr. Clin. Neurophysiol. 87, 128-143 (1993).

61. Peirce, J. W. PsychoPy-Psychophysics software in Python. J. Neurosci. Methods 162, 8-13 (2007).

62. Kaminski, J. A., Korb, F. M., Villringer, A. \& Ott, D. V. M. Transcranial magnetic stimulation intensities in cognitive paradigms. PLoS One 6, e24836 (2011).

63. Stanislaw, H. \& Todorov, N. Calculation of signal detection theory measures. Behav. Res. Methods, Instruments, \{\&\} Comput. 31, 137-149 (1999)

64. Ulrich, R. \& Miller, J. Effects of outlier exclusion on reaction time analysis. J. Exp. Psychol. Gen., https://doi.org/10.1037//00963445.123.1.34 (1991).

65. Haatveit, B. C. et al. The validity of $\mathrm{d}$ prime as a working memory index: results from the 'Bergen n-back task'. J. Clin. Exp. Neuropsychol. 32, 871-880 (2010).

66. Dienes, Z. Using Bayes to get the most out of non-significant results. Front. Psychol. 5, 781 (2014).

67. Wagenmakers, E., Wetzels, R., Borsboom, D. \& van der Maas, H. L. J. Why psychologists must change the way they analyze their data: The case of psi: Comment on Bem (2011). J. Pers. Soc. Psychol. 100, 426-432 (2011).

68. JASP Team. JASP (Version 0.8.3.1) [Computer software] (2017).

\section{Acknowledgements}

The study was supported by the National Brain Research Program (Grant No. KTIA_13_NAP-A-II/20) and by the Human Resource Development Operational Programme (Grant No. EFOP-3.6.1-16-2016-00008) providing support for study equipment and conduction. A.M. received support from the Bolyai Scholarship Program of the Hungarian Academy of Sciences. A.M. was supported by the UNKP-17-4 and T.V. by the UNKP-17-3 New National Excellence Program of the Ministry of Human Capacities during study conduction and manuscript preparation. We are grateful to D.N. for his incredibly useful comments on the drafts of the manuscript.

\section{Author Contributions}

T.V. participated in data acquisition, data management, analyzed data and wrote the manusript; V.L.N. designed the study and participated in data acquisiton; A.H. participated in data acquisition, K.K. participated in data acquisition, Zs.T.K. designed the study; L.V. designed the study, A.M. designed the study and wrote the manuscript.

\section{Additional Information}

Supplementary information accompanies this paper at https://doi.org/10.1038/s41598-018-33187-3.

Competing Interests: The authors declare no competing interests.

Publisher's note: Springer Nature remains neutral with regard to jurisdictional claims in published maps and institutional affiliations. 
(1) Open Access This article is licensed under a Creative Commons Attribution 4.0 International License, which permits use, sharing, adaptation, distribution and reproduction in any medium or format, as long as you give appropriate credit to the original author(s) and the source, provide a link to the Creative Commons license, and indicate if changes were made. The images or other third party material in this article are included in the article's Creative Commons license, unless indicated otherwise in a credit line to the material. If material is not included in the article's Creative Commons license and your intended use is not permitted by statutory regulation or exceeds the permitted use, you will need to obtain permission directly from the copyright holder. To view a copy of this license, visit http://creativecommons.org/licenses/by/4.0/.

(C) The Author(s) 2018 


\section{Supplementary Material}

Continuous theta-burst stimulation over the dorsolateral prefrontal cortex inhibits improvement on a working memory task

Teodóra Vékony ${ }^{\mathrm{*}}$, Viola Luca Németh ${ }^{\mathrm{a}}$, Adrienn Holczer ${ }^{\mathrm{a}}$, Krisztián Kocsis ${ }^{\mathrm{a}}$, Zsigmond Tamás

Kincses $^{\mathrm{a}}$, László Vécsei ${ }^{\mathrm{a}, \mathrm{b}}$, Anita Must ${ }^{\mathrm{b}, \mathrm{c} *}$

${ }^{\text {a }}$ Department of Neurology, University of Szeged, Szeged, Hungary

${ }^{\mathrm{b}}$ MTA-SZTE Neuroscience Research Group, Szeged, Hungary

${ }^{\mathrm{c}}$ Institute of Psychology, University of Szeged, Szeged, Hungary

${ }^{*}$ Corresponding authors:

Anita Must MD PhD, Institute of Psychology, University of Szeged, Egyetem u. 2, H-6722, Szeged, Hungary, must.anita@med.u-szeged.hu

Teodóra Vékony, Department of Neurology, University of Szeged, Semmelweis u. 6, H-6725, Szeged, Hungary, vekony.teodora@med.u-szeged.hu

\begin{tabular}{lcccccc}
\hline & $\mathrm{DF}$ & $\mathrm{Error} \mathrm{DF}$ & $\mathrm{F}$ & $\mathrm{p}$ & $\mathrm{n}_{\mathrm{p}}{ }^{2}$ & $\mathrm{BF}_{10}$ \\
\hline GROUP & 2 & 48 & 2.993 & .06 & .111 & .722 \\
GROUP $\times$ SIDE & 2 & 48 & .471 & .627 & .019 & .075 \\
GROUP $\times$ LOAD & 3.436 & 82.474 & .537 & .682 & .019 & .062 \\
GROUP $\times$ SIDE $\times$ LOAD & 3.925 & 94.190 & .482 & .627 & .035 & .086 \\
\hline
\end{tabular}

Supplementary Table S1. Comparison of pre-stimulation median RTs between groups

\begin{tabular}{lcccccc}
\hline & DF & Error DF & $\mathrm{F}$ & $\mathrm{p}$ & $\mathrm{n}_{\mathrm{p}}^{2}$ & $\mathrm{BF}_{10}$ \\
\hline GROUP & 2 & 48 & 2.125 & .131 & .048 & $<.001$ \\
GROUP $\times$ SIDE & 2 & 48 & .586 & .560 & .024 & .190 \\
GROUP $\times$ LOAD & 2 & 48 & .065 & .937 & .003 & .105 \\
GROUP $\times$ SIDE $\times$ LOAD & 2 & 48 & .135 & .375 & .040 & $<.001$ \\
\hline
\end{tabular}

Supplementary Table S2. Comparison of pre-stimulation d' scores between groups 


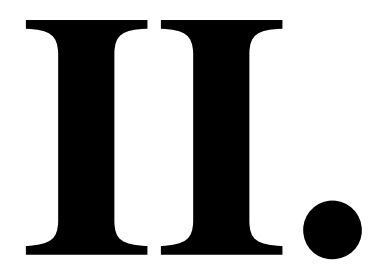




\title{
When less is more: Enhanced statistical learning of non-adjacent dependencies after disruption of bilateral DLPFC
}

\author{
Géza Gergely Ambrus ${ }^{\mathrm{a}, 1}$, Teodóra Vékony ${ }^{\mathrm{b}, 1}$, Karolina Janacsek ${ }^{\mathrm{c}, \mathrm{d}, \mathrm{e}, 1}$, Anna B.C. Trimborn ${ }^{\mathrm{f}, 1}$, \\ Gyula Kovács ${ }^{\mathrm{a}, 1}$, Dezso Nemeth ${ }^{\mathrm{c}, \mathrm{d}, \mathrm{g}_{*},}$ \\ ${ }^{a}$ Institute of Psychology, Friedrich Schiller University Jena, Leutragraben 1, D-07743 Jena, Germany \\ ${ }^{\mathrm{b}}$ Department of Neurology, University of Szeged, Semmelweis utca 6., H-6725 Szeged, Hungary \\ ${ }^{\mathrm{c}}$ Institute of Psychology, Eötvös Loránd University, Izabella utca 46., H-1064 Budapest, Hungary \\ ${ }^{\mathrm{d}}$ Brain, Memory and Language Research Group, Institute of Cognitive Neuroscience and Psychology, Research Centre for Natural Sciences, Hungarian Academy of Sciences, \\ Magyar tudósok körútja 2., H-1117 Budapest, Hungary \\ ${ }^{\mathrm{e}}$ School of Human Sciences, Faculty of Education, Health and Human Sciences, University of Greenwich, Old Royal Naval College, Park Row, SE10 9LS London, United \\ Kingdom \\ ${ }^{\mathrm{f}}$ Donders Institute for Brain, Cognition and Behaviour, Radboud University, Montessorilaan 3, 6525 HR Nijmegen, the Netherlands \\ ${ }^{\mathrm{g}}$ Lyon Neuroscience Research Center (CRNL), INSERM, CNRS, Université Claude Bernard Lyon 1, 95 Boulevard Pinel, 69675 Bron, France
}

\section{A R T I C L E I N F O}

\section{Keywords:}

Non-adjacent dependency

Statistical learning

Probabilistic sequence learning

Dorsolateral prefrontal cortex (DLPFC)

Memory consolidation

Transcranial magnetic stimulation (TMS)

\begin{abstract}
A B S T R A C T
Brain networks related to human learning can interact in cooperative but also competitive ways to optimize performance. The investigation of learning and memory processes in a competitive framework is still rare. Previous studies have shown that manipulations reducing the engagement of prefrontal cortical areas could lead to improved statistical learning performance. However, no study has investigated how disruption of the dorsolateral prefrontal cortex (DLPFC) affects the acquisition and consolidation of non-adjacent second-order dependencies. The present study aimed to test the role of the DLPFC, more specifically, the Brodmann 9 area in implicit temporal statistical learning of non-adjacent dependencies. We applied $1 \mathrm{~Hz}$ inhibitory transcranial magnetic stimulation or sham stimulation over both the left and right DLPFC intermittently during the learning. The DLPFC-stimulated group showed better performance compared to the sham group after a 24-hour consolidation period. This finding suggests that the disruption of DLPFC during learning induces qualitative changes in the consolidation of non-adjacent statistical regularities. A possible mechanism behind this result is that the stimulation of the DLPFC promotes a shift to model-free learning by weakening the access to model-based processes.
\end{abstract}

\section{Introduction}

Accumulating evidence supports the existence of interactive learning/memory processes, which can be either cooperative or competitive. Such a competitive relationship is theorized to exist between the deliberative model-based and the incidental/reflexive model-free processes underlying learning (Daw, Niv, \& Dayan, 2005). Model-based processes refer to the more controlled forms of learning that include the development of complex representations based on testing hypotheses about the environment (Beierholm, Anen, Quartz, \& Bossaerts, 2011; Wan Lee, Shimojo, \& O’Doherty, 2014). Consequently, internal models are formulated, which may be used both for the already learned and the newly encountered structures (Daw et al., 2005; Haith \& Krakauer,
2013). These processes were associated with goal-directed control, processing speed, executive functions, and working memory (e.g., Kurth-Nelson, Bickel, \& Redish, 2012; Otto, Raio, Chiang, Phelps, \& Daw, 2013; Schad et al., 2014). Model-free processes refer to habit-like, associative forms of learning whereby one extracts predictable structural regularities from the environment without intention or conscious monitoring. Thus, this type of learning is stimulus-driven and typically occurs implicitly. This predictive processing and capacity to detect patterns are crucial for aspects of statistical learning, which are involved in the acquisition of cognitive, social, and motor skills and habits (e.g., Kaufman et al., 2010; Lieberman, 2000; Nemeth \& Janacsek, 2011). Disruptive stimulation of the dorsolateral prefrontal cortex (DLPFC) was shown to shift the balance of model-free and model-based processes

\footnotetext{
* Corresponding author at: Lyon Neuroscience Research Center, Université Claude Bernard Lyon 1, 95 Boulevard Pinel, 69675 Bron, France.

E-mail address: dezso.nemeth@univ-lyon1.fr (D. Nemeth).

${ }^{1}$ These authors contributed equally to this work.
} 


\section{Experimental design. A. The ASRT task B. Experimental procedure}

A

\author{
Stimuli of the ASRT task
}

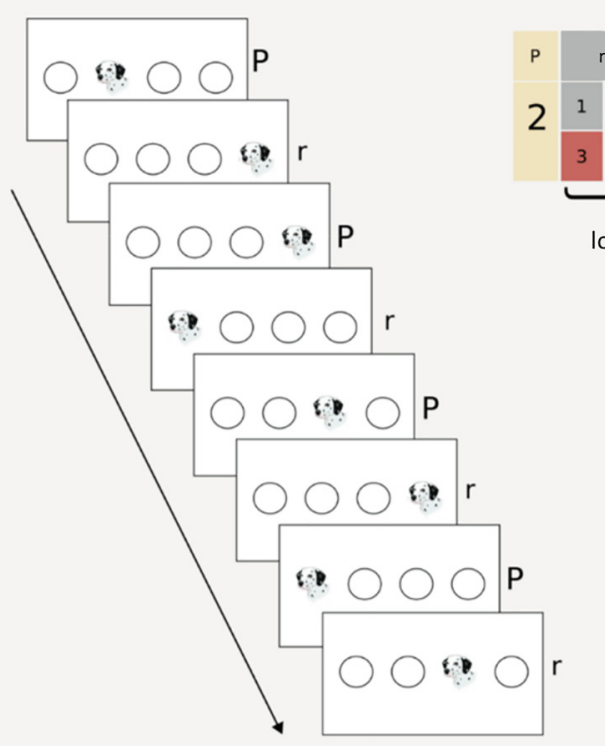

Sequence: e.g., $2 r 4 r 3 r 1 r$

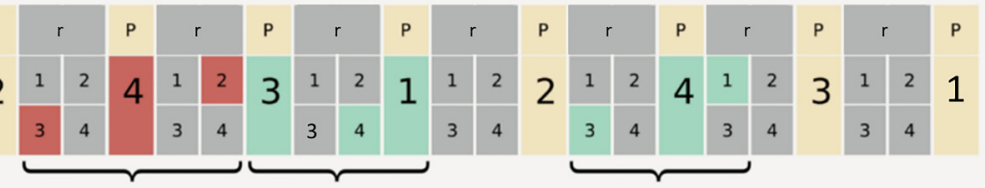

low-probability high-probability triplet triplet $r-P-r \quad P-r-P$

high-probability triplet $r-P-r$

B

\begin{tabular}{|c|c|c|}
\hline & $\begin{array}{c}\text { Structure: } \\
P-r-P\end{array}$ & $\begin{array}{c}\text { Structure: } \\
r-P-r\end{array}$ \\
\hline $\begin{array}{l}\text { High-probability } \\
\text { triplets } \\
62.5 \% \text { of all trials }\end{array}$ & $3-4-1(50 \%)$ & $3-4-1(12.5 \%)$ \\
\hline $\begin{array}{l}\text { Low-probability } \\
\text { triplets } \\
37.5 \% \text { of all trials }\end{array}$ & $\begin{array}{l}\text { never occurring } \\
\text { (always high) }\end{array}$ & $\begin{array}{l}3-4-2(12.5 \%) \\
3-4-3(12.5 \%) \\
3-4-4(12.5 \%)\end{array}$ \\
\hline
\end{tabular}

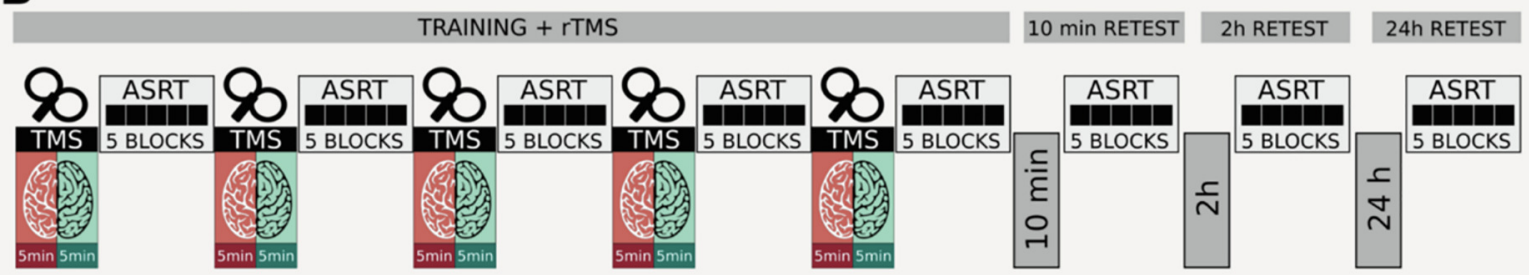

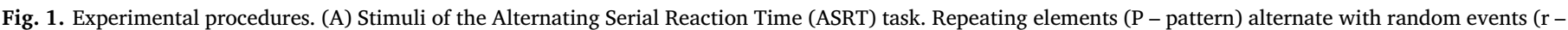

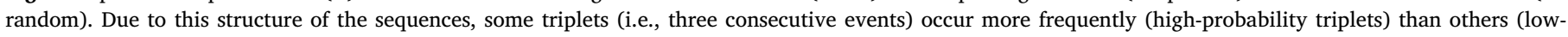

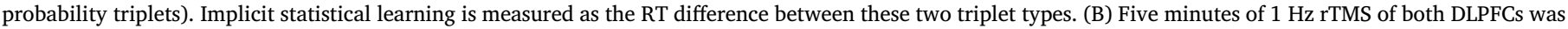

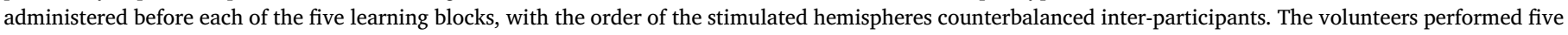
ASRT blocks $10 \mathrm{~min}, 2 \mathrm{~h}$, and $24 \mathrm{~h}$ post-learning, as well.

to the benefit of model-free processes during reinforcement learning (Smittenaar, FitzGerald, Romei, Wright, \& Dolan, 2013). Congruently, better statistical learning was associated with weaker model-based processes (Janacsek, Fiser, \& Nemeth, 2012; Nemeth, Janacsek, Polner, \& Kovacs, 2013; Tóth et al., 2017; Virag et al., 2015), supporting the potential competition between the two types of learning processes. Can "less" involvement of the DLPFC be "more" beneficial for cognitive functions driven by model-free processes? Our study aimed to answer this question by directly manipulating the involvement of DLPFC in temporally distributed statistical learning using repetitive transcranial magnetic stimulation (rTMS).

Previous research has proposed that the neural circuitry involving parts of the basal ganglia, notably the dorsolateral striatum, supports model-free learning processes. The circuitry involving medial temporal lobe structures, including the hippocampus, and the areas of the default network promotes model-based processes (Buckner \& DiNicola, 2019; Daw, Gershman, Seymour, Dayan, \& Dolan, 2011; Dayan \& Berridge, 2014; Janacsek et al., 2012; Packard \& Knowlton, 2002; Vikbladh et al., 2019; Wunderlich, Smittenaar, \& Dolan, 2012; Yin, Knowlton, \& Balleine, 2004). Studies suggest that both processes involve the lateral prefrontal cortical regions that subserve several cognitive functions, such as executive functions, working memory, memory encoding, and access to long-term memory (Baier et al., 2010; Blumenfeld \& Ranganath, 2007; Culbreth, Westbrook, Daw, Botvinick, \& Barch, 2016;
Koechlin \& Summerfield, 2007; Lara \& Wallis, 2015; McNab \& Klingberg, 2008; Otto et al., 2013; Otto, Skatova, Madlon-Kay, \& Daw, 2014). The neural basis of picking up probabilistic statistical regularities has been investigated by multiple neuroimaging studies. For example, Simon, Vaidya, Howard, and Howard (2012) used event-related functional magnetic resonance imaging during a probabilistic sequence learning task. The study provided evidence for the role of the hippocampus in the early stage of learning and the caudate in later stages. Using diffusion tensor imaging, Bennett, Madden, Vaidya, Howard, and Howard (2011) found that the integrity of the neural tracts between the DLPFC and the hippocampus, and also between the DLPFC and the caudate nucleus are related to the degree of statistical learning. Stillman et al. (2013) found that a functional connectivity index between the caudate and medial temporal lobe showed a positive correlation with probabilistic statistical learning. Based on their findings, the authors also stressed the potential mediating role of the DLPFC between other, statistical learning-related areas such as the caudate and the medial temporal regions. Thus, we hypothesized that the DLPFC might modulate statistical learning abilities by being a part of the neural circuits supporting the model-based and model-free processes.

Employing non-invasive brain stimulation methods, we can reveal relationships between learning and brain areas (and their related networks). To date, only a few studies have investigated the acquisition of temporally distributed deterministic or probabilistic regularities (often 
termed statistical learning as well) by stimulating the DLPFC. An early rTMS study by Pascual-Leone, Wassermann, Grafman, and Hallett (1996) showed that $5 \mathrm{~Hz}$ rTMS over the contralateral DLPFC during a deterministic serial reaction time task (SRTT) impairs online learning. Galea, Albert, Ditye, and Miall (2010) applied inhibitory continuous theta-burst stimulation (cTBS) over the DLPFC following practice on SRTT. After an 8-hour consolidation period, subjects were faster on sequence compared to random elements in the verum, but not in the sham group. Smalle, Panouilleres, Szmalec, and Möttönen (2017) found increased learning on phonological sequences following cTBS over the left DLPFC. Contrary, Savic, Cazzoli, Müri, and Meier (2017) reported null effects of different brain stimulation methods over the DLPFC on a deterministic sequence learning task that requires the use of only one hand. This result suggests that, in some cases, a robust interhemispheric compensation might obscure the effects of the stimulation. More importantly, all the mentioned studies used deterministic sequences with adjacent regularities instead of probabilistic sequences with non-adjacent regularities (Remillard, 2008). In non-adjacent statistical learning, the predictable information is hidden in noise within the stimulus stream. Therefore, the task mimics more closely the acquisition of real-life skills (for example, language learning, Christiansen \& Chater, 2015), which occurs under uncertainty, in a noisy environment (Fiser, Berkes, Orbán, \& Lengyel, 2010). The role of the DLPFC in this complex, ecologically valid form of statistical learning remains unclear.

In this study, we go beyond previous findings by disrupting the DLPFC during a non-adjacent statistical learning task using bilateral stimulation. We aimed to reveal whether less involvement of the DLPFC could be more beneficial for the acquisition and consolidation of statistical learning skills. To assess statistical learning, we chose a widely used probabilistic sequence learning task, namely the Alternating Serial Reaction Time (ASRT) task (Fig. 1A). This task has been used previously in experimental psychology (Howard \& Howard, 1997; Howard et al., 2004; Kóbor, Janacsek, Takács, \& Nemeth, 2017; Nemeth et al., 2010; Song, Howard, \& Howard, 2007), developmental (Janacsek et al., 2012; Juhasz, Nemeth, \& Janacsek, 2019; Nemeth, Janacsek, \& Fiser, 2013) as well as neuroimaging studies (Bennett et al., 2011; Stillman et al., 2013). The ASRT task is a four-choice reaction time task in which predetermined stimuli alternate with random elements, creating a probabilistic structure with more probable versus less probable stimulus triplets. Participants can pick up these non-adjacent statistical regularities: over time, performance differences emerge between highand low-probability triplets without the participants becoming aware of the underlying structure (Howard \& Howard, 1997; Howard et al., 2004). During the Training/rTMS session, we used $1 \mathrm{~Hz}$ repetitive TMS over the DLPFC, which was shown to decrease activity in the targeted brain area (Groiss, Ugawa, Paulus, \& Huang, 2012) extending beyond the termination of the stimulation (Robertson, Théoret, \& PascualLeone, 2003; Walsh \& Cowey, 2000). As ASRT requires the use of both hands, bilateral stimulation might be an ideal choice to control for the possible premotor response bias (by bilateral stimulation, high- and low-probability triplets are affected to a similar extent). Moreover, a sequential bilateral TMS protocol was chosen to suppress the compensation of the non-stimulated hemisphere. Retest sessions $10 \mathrm{~min}, 2 \mathrm{~h}$, and $24 \mathrm{~h}$ after the termination of the training were implemented in the design. Our purpose was to study the effect of TMS on the whole learning process and to differentiate between the immediate and longlasting results of stimulation and its impact on the offline consolidation of acquired knowledge. We hypothesized that disrupting the DLPFC bilaterally would increase statistical learning performance in the ASRT task.

\section{Materials and methods}

\section{Participants}

Thirty-two participants took part in the experiment. All of them were right-handed; their visual acuities were normal or corrected to normal. None of the participants reported a history of neurological or psychological disorders, drug or alcohol abuse, had metal implants, or were taking regular medication relevant to the study. Written informed consent was acquired from all participants. All participants tolerated the experimental procedures, and none withdrew because of discomfort with TMS. All participants were students of the University of Jena and participated in exchange for partial course credits or monetary compensation. One participant was excluded from the final sample because of poor performance on the Berg Card Sorting Test (\% of preservative errors $=31.25 \%$; $\%$ of correct responses: $51.56 \%$; the scores were more than three standard deviations above the mean, see group averages in the Experimental Procedure section). Thus, the final sample contained data from 31 participants (16 in the DLPFC Group and 15 in the Sham Group, four males, $\mathrm{M}_{\mathrm{age}}=22.16$ years, $\mathrm{SD}_{\text {age }} \pm 3.01$ ). The experiment was conducted in accordance with the guidelines of the Declaration of Helsinki and with the approval of the ethics committee of the University of Jena.

\section{Alternating serial reaction time task}

Statistical learning was measured using the Alternating Serial Reaction Time (ASRT) Task (Howard et al., 2004; Song et al., 2007). In this task, a stimulus (a dog's head) appeared in one of four horizontally arranged empty circles on the screen. Participants were instructed to press the corresponding key (Z, C, B, and M on a QWERTY keyboard), as quickly and as accurately as possible (Fig. 1A). The buttons $\mathrm{Z}$ and $\mathrm{C}$ had to be pressed by the middle and index fingers of the left hand. The $B$ and $\mathrm{M}$ buttons had to be pressed by the index and middle fingers of the right hand, respectively. The target remained on the screen until the participant pressed the correct button. The response-to-stimulus interval was set to $120 \mathrm{~ms}$. Stimuli were presented in blocks of 85 trials. The first five trials of each block were random elements and for practice purposes only (not analyzed further). After these five practice trials, an eight-element alternating sequence was repeated ten times in a block (e.g., $2 r 4 r 3 r 1 r$, where 1-4 indicate the target locations from left to right, and $r$ indicates a randomly selected position out of the four possible ones) (Fig. 1A). The predetermined order of the pattern elements remained unknown to the participants. Due to the alternation of random and pattern elements, some runs of three consecutive items (henceforth referred to as triplets) occurred with higher probability than other ones. We refer to these types of stimuli as high-probability and low-probability triplets, respectively. For example, considering the above illustration, 2_4, 4_3, 3_1, and 1_2 (where "." indicates the middle element of a triplet) occur with high-probability, because the third element (bold number) could be derived from a (predetermined) pattern, and in some cases, from random items. On the contrary, 1_3 and $4 \_1$ occur with less probability because in that case, the third element could only be random. Therefore, the third event of a highprobability triplet is more predictable from the first element than in the case of the low-probability triplets. Accordingly, each of the trials of the ASRT was categorized as either the third element of a high- or a lowprobability triplet. Overall, 64 possible triplets can occur in the task, of which 16 are high-probability triplets, each of them occurring in approximately $4 \%$ of the trials $(62.5 \%$ in total). Each of the remaining 48 triplets occurred in around $0.8 \%$ of the trials (37.5\% in total). Thus, the high-probability triplets occur five times more often than the lowprobability triplets.

\section{Structural MRI and neuronavigated TMS}

Structural MRI scanning was performed using a Siemens Magnetom Trio 3 T MRI scanner at the Institute for Diagnostic and Interventional Radiology, University of Jena. High-resolution sagittal T1-weighted images for the 3D head and brain meshes were acquired using a magnetization EPI sequence (MP-RAGE; TR $=2300 \mathrm{~ms}$; TE $=3.03 \mathrm{~ms}$; 
$1 \mathrm{~mm}$ isotropic voxel size). For neuronavigated TMS, the 3D-head and brain models were created from the participants' individual MRI scans. Coordinates for the DLPFC were taken from a meta-analysis by Cieslik et al. (2013), which corresponds to the dorsal part of the DLPFC (MNI coordinates: $\mathrm{x}=37, \mathrm{y}=33, \mathrm{Z}=32$ ). This area was revealed to be involved in executive control and working memory processes. For sham TMS, the coil was oriented perpendicularly, facing away from the skull (Lisanby, Gutman, Luber, Schroeder, \& Sackeim, 2001).

TMS stimulation was delivered using a PowerMag 100 Research Stimulator (MES Forschungssysteme $\mathrm{GmbH}$ ). Neuronavigation was carried out using a PowerMag View (MES Medizintechnik GmbH) Neuronavigation system. Magnetic pulses were delivered at $1 \mathrm{~Hz}$, at $55 \%$ maximum stimulator output. A single intensity was used based on previous studies (Figner et al., 2010; Silvanto, Cattaneo, Battelli, \& Pascual-Leone, 2008). TMS was applied before each of the five learning blocks, that is, before the first block and in the inter-block intervals (300 pulses, $5 \mathrm{~min}$ per hemisphere). The order in which the two hemispheres were stimulated was counterbalanced inter-participants.

\section{Experimental procedures}

Participants were seated in a dimly lit room; their heads were fixed using a chinrest, $60 \mathrm{~cm}$ viewing distance away from the stimulus presentation monitor. After giving informed consent, the volunteers performed an ASRT practice run to familiarize themselves with the task and the keyboard layout. In the Training/rTMS session, the participants received $1 \mathrm{~Hz}$ rTMS over both left and right hemispheres sequentially ( $5 \mathrm{~min}, 300$ TMS pulses for each hemisphere; thus, $5 \mathrm{~min}$ for the left and after that the right, in a counterbalanced order inter-participants), then performed five blocks of the ASRT task, lasting approximately $5 \mathrm{~min}$. This procedure was repeated five times. Therefore, a total of 25 blocks of ASRT were completed, which provides enough trials for learning to manifest as shown by previous studies (Janacsek, Fiser, \& Nemeth, 2012; Nemeth et al., 2010; Vékony et al., 2020). The order in which the two hemispheres were stimulated was assigned randomly, remained the same for each participant. To test the role of the DLPFC in statistical learning in both the acquisition and the consolidation, we tested ASRT performance multiple times after the stimulation as well. In the retest sessions, the participants performed five blocks of the ASRT task $10 \mathrm{~min}, 2 \mathrm{~h}$, and $24 \mathrm{~h}$ after the completion of the Training/rTMS session (Fig. 1B). The exact retest times followed previous literature (Janacsek, Ambrus, Paulus, Antal, \& Nemeth, 2015). The 10 min retest session aimed to test the immediate aftereffect of TMS without preceding stimulation. With the help of the $2 \mathrm{~h}$ retest session, we could test the long-term effects of the stimulation. The performance after $24 \mathrm{~h}$ was thought to provide information about the impact of the stimulation on consolidation.

To ensure that the two experimental groups did not differ in executive function performance, we administered the short-form of the Berg Card Sorting Test (Fox, Mueller, Gray, Raber, \& Piper, 2013) and the Counting Span test (Case, Kurland, \& Goldberg, 1982; Conway et al., 2005; Engle, Laughlin, Tuholski, \& Conway, 1999) after the completion of the ASRT in the last session. We observed no significant differences in performance between the two experimental groups (Berg Card Sorting Test, percent correct responses: DLPFC: $\mathrm{M}=81.06$, $\mathrm{SD}=5.90$; Sham: $\mathrm{M}=80.42, \mathrm{SD}=7.76, p=.80$; percent perseverative errors: $\mathrm{DLPFC}: \mathrm{M}=10.16, \mathrm{SD}=4.56$; Sham: $\mathrm{M}=11.67$, $\mathrm{SD}=4.83, p=.38$; percent non-perseverative errors: DLPFC: $\mathrm{M}=8.79, \mathrm{SD}=5.59$; Sham: $\mathrm{M}=7.92, \mathrm{SD}=5.06, p=.65$; Counting span, mean of three runs: DLPFC: $\mathrm{M}=4.06, \mathrm{SD}=1.11$, Sham: $\mathrm{M}=3.62$, SD $=0.85, p=.23$ ).

As a part of the post-experimental debriefing, the participants filled out a questionnaire assessing their levels of discomfort, tiredness, and perceived task difficulty, measured on a ten-point scale.

The Training/rTMS session (with informed consent) lasted approximately $2 \mathrm{~h}$, the $10 \mathrm{~min}$ and $2 \mathrm{~h}$ retest sessions lasted $5 \mathrm{~min}$ each, and the $24 \mathrm{~h}$ retest session (with the control tasks) and debriefing lasted approximately $30 \mathrm{~min}$.

\section{Statistical analysis}

Only correct responses were considered for the ASRT analysis, and stimulus repetitions (e.g., 333, 444) and trills (e.g., 313, 121) were also excluded (Howard \& Howard, 1997; Howard et al., 2004). Trials with reaction times (RTs) more than 2.5 standard deviations above or below the mean of the given epoch were eliminated (separately for each participant). After that, we calculated the mean RTs for high- and lowprobability triplets separately. Implicit statistical learning was assessed using a triplet-learning index, calculated by subtracting the RTs for high-probability triplets from those for low-probability triplets. To control for the non-specific effects of time and the possible individual differences in RTs, we calculated a percentage learning index as follows: [(RTs for low-probability triplets - RTs for high-probability triplets)/RTs for low-probability triplets)]. A higher learning index thus means faster responses for high- than for low-probability triplets, that is, better statistical learning performance.

The learning index was calculated for each session of the experiment for each participant. We conducted a 2 (Group: DLPFC Stimulation vs. Sham Stimulation) $\times 4$ (Session: Training/rTMS session vs. 10 min retest session vs. 2 h retest session vs. 24 h retest session) mixed-design ANOVA to compare the statistical learning performance between the two stimulation groups throughout the experiment. Greenhouse-Geisser corrections were applied where necessary. Significant main effects and interactions were further analyzed using Bonferroni-corrected post-hoc comparisons.

As we aimed to investigate the effects of rTMS on both hemispheres, we tested whether the observed effect was due to the stimulation of the hemisphere just before the particular ASRT block. Thus, we conducted a 2 (Order: Right Start vs. Left Start) $\times 4$ (Session: Training/rTMS session vs. 10 min retest session vs. $2 \mathrm{~h}$ retest session vs. $24 \mathrm{~h}$ retest session) ANOVA on the learning index to ascertain if our results were influenced by the stimulation order.

We also performed an analysis of the ASRT performance across the four sessions with the raw RTs for high- and low-probability triplets as dependent variables. To see how the initial learning was affected by the stimulation, we analyzed the learning indices of the five epochs of the Training/rTMS session. We also compared the learning indices of the three retest sessions to the last epoch of the Training/rTMS session (see details of these analyses and results in the Supplementary Materials).

All analyses were two-tailed and were conducted with a significance level of $p<.05$.

\section{Results}

The ANOVA conducted on the learning indices across sessions revealed a significant main effect of the experimental Session, $F_{3,87}=7.11, p<.001, \eta_{p}{ }^{2}=.20$. The pairwise comparisons of this main effect indicate an overall increase in performance in all three retest sessions when compared to the Training/rTMS session (Training/ rTMS session vs. 10 min retest session: $p=.001,2$ h retest session: $p=.001,24$ h retest session: $p=.004)$. There was no main effect of Group, $F_{1,29}=0.61, p=.44, \eta_{p}^{2}=.02$, but the interaction between the experimental Session and Group was shown to be significant, $F_{3}$, ${ }_{87}=3.96, p=.01, \eta_{p}{ }^{2}=.12$. In the Sham Group, pairwise comparisons revealed an increased performance at the 10 min retest session compared to the Training/rTMS session $(p=.002)$. Performance in the DLPFC Group also increased with time compared to the Training/rTMS session ( $2 \mathrm{~h}$ retest session: $p=.01$ ), and contrary to the Sham Group, the difference remained significant at the $24 \mathrm{~h}$ retest session $(p=.001)$ (Fig. 2A). The comparisons of the learning indices between the two groups revealed that the Sham and DLPFC Groups showed similar learning indices during the Training/rTMS, $10 \mathrm{~min}$, and $2 \mathrm{~h}$ sessions (all 
A

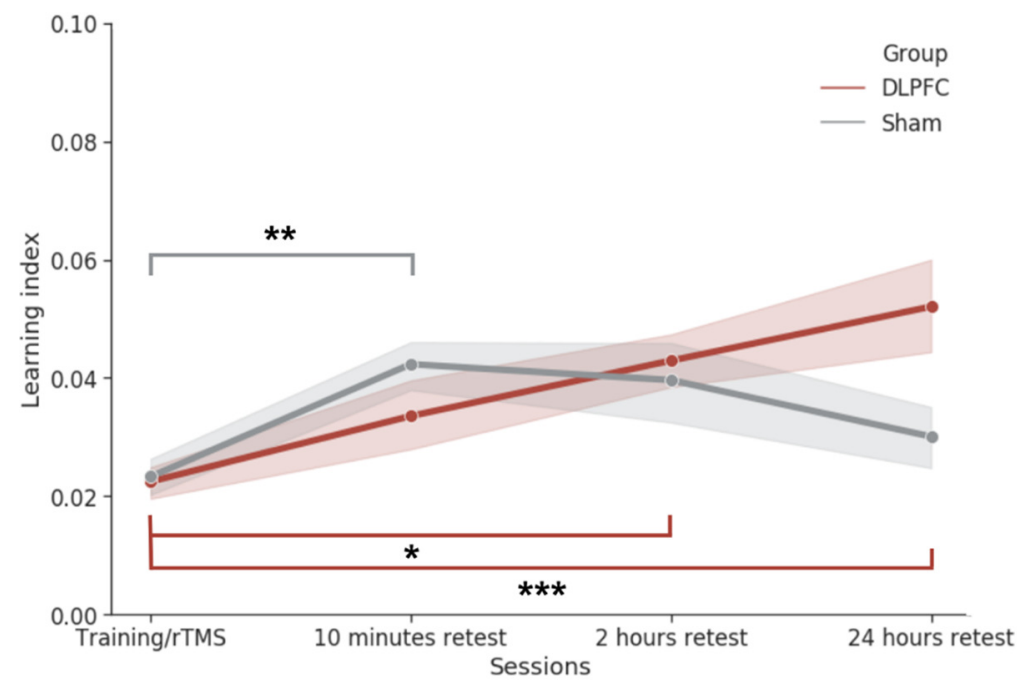

B

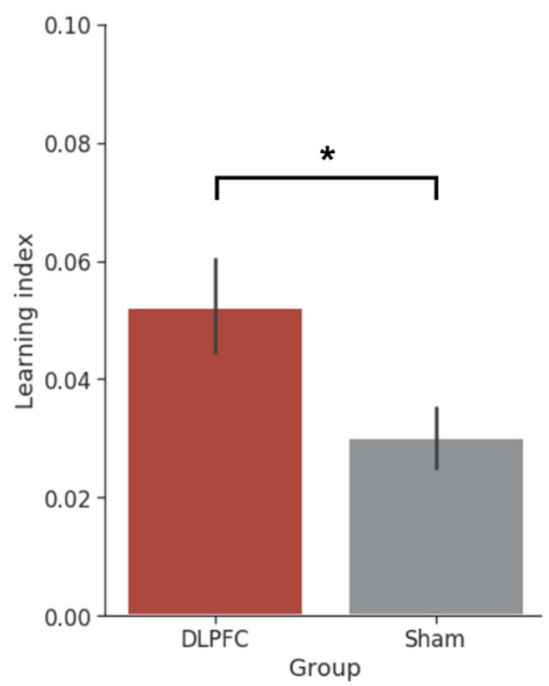

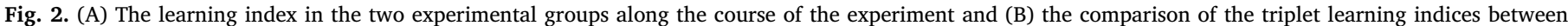

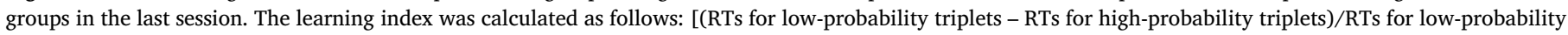

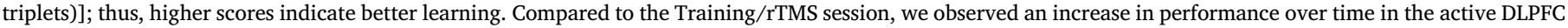

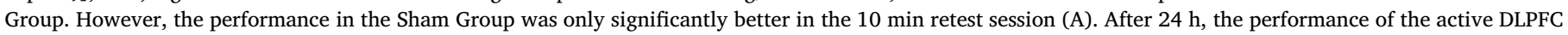
Group was statistically better that of the Sham Group (B). The error bars denote the SEM. *: $p<.05,{ }^{* *}: p<.01,{ }^{* * *}: p=.001$.

$p>$.25). However, the DLPFC Group showed a significantly greater learning index than the Sham Group at the $24 \mathrm{~h}$ retest session $(p=.03)$ (Fig. 2B). The stimulation seemed to affect the RTs for the low-probability triplets primarily; see details in the first section of the Supplementary Materials.

The observed group differences are unlikely to be due to a general effect of the stimulation on arousal level because the general reaction time (Session $\times$ Group interaction: $F_{3,87}=0.39, p=.76, \eta_{p}^{2}=.01$ ) and response accuracy (Session $\times$ Group interaction: $F_{3,87}=0.38, p=.77$, $\eta_{p}^{2}=.01$ ) was not statistically different between the two groups. Furthermore, the level of discomfort $(p=.57)$, tiredness $(p=.83)$, perceived task difficulty $(p=.24)$, assessed as a part of the post-experiment debriefing, was also not different between the DLPFC and the Sham Group (see Methods).

Moreover, the order of the hemispheres stimulated, assessed by the two-way interaction of a 2 (Order: Right Start vs. Left Start) $\times 4$ (Session: Training/rTMS session vs. 10 min retest session vs. $2 \mathrm{~h}$ retest session vs. $24 \mathrm{~h}$ retest session) mixed-design ANOVA, did not affect statistical learning performance, $F_{3,42}=0.15, p=.93, \eta_{p}^{2}=.01$.

\section{Discussion}

To date, only a few studies have investigated the role of the DLPFC in statistical learning (Galea et al., 2010; Pascual-Leone et al., 1996; Savic et al., 2017; Smalle et al., 2017), while none of them tested its role in the acquisition and consolidation of non-adjacent dependencies. Here, we aimed to fill this gap by administering bilateral rTMS over the DLPFCs during temporal statistical learning of non-adjacent dependencies. We went beyond previous studies in three aspects. First, instead of deterministic sequences, we tested the effect of rTMS on the acquisition of non-adjacent probabilities. Second, we applied bilateral stimulation to control for a possible interhemispheric compensation of the non-stimulated hemisphere. Third, we tested the effect of the TMS protocol used throughout the training session as well as $10 \mathrm{~min}, 2 \mathrm{~h}$, and $24 \mathrm{~h}$ post-training. Our results show that the bilateral disruption of the DLPFCs during the training session has a beneficial effect on the statistical learning performance after $24 \mathrm{~h}$. Therefore, we suggest that DLPFCs play a role in non-linguistic statistical learning processes. As predicted, our findings are in line with the competition model that posits an antagonistic relationship between model-based and modelfree learning processes (Daw et al., 2011; Janacsek et al., 2012; Nemeth et al., 2013; Smittenaar et al., 2013; Virag et al., 2015).

In agreement with the computational framework of model-free and model-based processes, previous research further demonstrated that cognitive functions that are mainly determined by these two types of processes have an inverse relationship on the behavioral level. For instance, Virag et al. (2015) showed a negative correlation between working memory/executive functions and implicit statistical learning. Filoteo, Lauritzen, and Maddox (2010) found that implicit category learning improved with the addition of a secondary working memory task, that is, with the reduction of the contribution of model-based learning processes (however, for a critical re-evaluation of this study see Newell, Moore, Wills, \& Milton, 2013). Nemeth et al. (2013) found increased statistical learning performance in hypnosis compared to an alert, awake state, by possibly reducing long-range brain connectivity (Fingelkurts, Fingelkurts, Kallio, \& Revonsuo, 2007; Oakley \& Halligan, 2009). Additionally, several developmental studies found that children perform better than adults on non-adjacent statistical learning tasks (Janacsek et al., 2012; Juhasz et al., 2019; Zwart, Vissers, Kessels, \& Maes, 2019). Supporting the competition model, the degree of learning decreases with the onset of adolescence, coinciding with the maturation of the dorsolateral regions of the prefrontal cortex (Bunge \& Zelazo, 2006; Gogtay et al., 2004; Kadosh, Heathcote, \& Lau, 2014; Thompson et al., 2001). Besides the above-mentioned behavioral studies, Tóth et al. (2017) found evidence for this inverse relationship at the level of neural oscillations. Namely, they detected increased statistical learning associated with weaker fronto-parietal connectivity in theta frequency, a band that plays a crucial role in memory access (Düzel, Penny, \& Burgess, 2010) and also in sentence processing and working memory (Beese, Meyer, Vassileiou, \& Friederici, 2017). These previous studies revealed only indirect, correlational relationships; however, our recent findings yield evidence for the role of the DLPFC in non-adjacent statistical learning.

To date, only four studies have investigated the role of the DLPFC on temporally distributed deterministic or probabilistic regularities using TMS protocols. Intending to disrupt cortical processing by $5 \mathrm{~Hz}$ rTMS during deterministic SRTT, Pascual-Leone et al. (1996) found that stimulation over the contralateral DLPFC impaired online learning. It 
should be noted that since the publication of this study, $5 \mathrm{~Hz}$ rTMS has been found to induce excitatory effects on cortical excitability (Matsunaga et al., 2005; Peinemann et al., 2004); thus, the performance decrease in this study might be better explained by the facilitation of DLPFC functions, in line with the competition account. To test the role of the DLPFC in the consolidation of sequential knowledge, Galea et al. (2010) applied disruptive cTBS after the execution of deterministic SRTT. They found an offline improvement following the inhibition of the right but not the left DLPFC, which they explained by interference between declarative and procedural consolidation processes. However, based on the results of Galea et al., we cannot decide whether a disrupted DLPFC during learning can affect the initial learning. Smalle et al. (2017) went beyond Galea et al. (2010) by applying disruptive stimulation over the left DLPFC prior to learning; increased learning on phonological sequences was found in the Hebb repetition paradigm. A follow-up analysis showed a negative correlation between learning performance and executive functions. To control for the possible compensation of the non-stimulated hemisphere, Savic et al. (2017) tested the effect of brain stimulation over the DLPFC on a deterministic sequence learning task, and no stimulation effect was found over either hemisphere. Taken together, most of the previous TMS studies (except Savic et al., 2017) point in the same direction: facilitating stimulation of the DLPFC hinders, while inhibitory stimulation improves the learning of new sequences, patterns, or statistical regularities.

Our results open up a new theoretical perspective in interpreting the role of the DLPFC in statistical learning. The DLPFC might have a role in model-based processes, such as in accessing the existing models or longterm memory representations, which might be "harmful" when learning of new patterns is required. This idea is supported by results showing that stronger executive functions that substantially involve the activation of the DLPFC might be associated with weaker statistical learning (Janacsek et al., 2012; Smalle et al., 2017; Virag et al., 2015). Moreover, already built models of the statistical regularities seem to hinder the adaptation to changes in those statistical regularities (Kóbor, Horváth, Kardos, Nemeth, \& Janacsek, 2019). If access to these modelbased processes is limited, then the learning process has to shift towards a model-free approach, which could lead to enhanced learning of entirely new patterns. This framework explains not only the results of the previously mentioned TMS studies on statistical learning well, but also our findings on the consolidation. Namely, as we disrupted the DLPFC, we found better performance after the 24-hour consolidation period. When the DLPFC is fully functioning, the model-free processes extract the statistical information from the stimulus stream, and the DLPFCmediated model-based processes contaminate these statistics with topdown information. In the offline period, this mixed information consolidates (Fig. 3). However, the stimulation of the DLPFC possibly interrupts the top-down information flow and its mixture with the datadriven extraction of pure statistical information. This pure statistical information consolidates, which is optimal when the brain faces the challenge of learning entirely new regularities. At the level of implementation, we suggest that these mechanisms might be realized by modulating the activation in the prefrontal-hippocampal circuitry. It was shown that the neural tracts between the DLPFC and the hippocampus are related to the degree of statistical learning (Stillman et al., 2013). For example, Ross, Brown, and Stern (2009) also showed the role of the hippocampus in the retrieval of learned sequences. A competitive relationship was also described between the hippocampus and striatum during sequence learning, whereby activity in the hippocampus decreases in parallel to an increase in the striatal area (Albouy et al., 2008, 2015). These competitive patterns were linked to performance gains after a consolidation period (Albouy, King, Maquet, \& Doyon, 2013). We hypothesize that the DLPFC might play a switching role in similar scenarios (Smittenaar et al., 2013; Stillman et al., 2013). By its disruption, the advantage in the competition changes in favor of the striatal areas. Thus, it can lead to a better consolidation of the statistical regularities because they are more involved in model-free processes.

We found an effect of stimulation only after a 24-hour consolidation period. Therefore, a major difference between our results and previous TMS studies is that they found immediate effects of stimulation (Pascual-Leone et al., 1996; Smalle et al., 2017). This difference may be explained by the differences between deterministic and probabilistic statistical learning tasks. In the ASRT task, the relevant information is hidden in the noise; therefore, the model-building process might be slower, and model-free learning might dominate the course of learning through a more extended period. Thus, if stimulation takes place before or during learning, the advantageous effects of reduced model-based processes might appear only at a later time point compared to deterministic tasks. Nevertheless, across-study differences in the stimulation intensity, the number of pulses, or the timing of the stimulation relative to the training session could also contribute to the inconsistencies in the observed aftereffects (Klomjai, Katz, \& Lackmy-Vallée, 2015; Thut \& Pascual-Leone, 2010). Systematic investigations of the role of the DLPFC in deterministic and probabilistic learning as well as the effects of different stimulation parameters on the learning process, will be required to clarify the inconsistencies in the outcome of stimulation.

We believe that our findings provide a broader theoretical perspective to the process-level understanding of statistical learning. In this view, the fine regulation of shifting between model-based and modelfree processes during learning and consolidation determines the quality of the acquired statistical knowledge. This theoretical framework is supported by evidence using linguistic stimuli (Smalle et al., 2017) and, through the present study, by perceptual-motor stimuli. Based on these findings, we speculate that this phenomenon is generalizable and we hypothesize its existence in most statistical learning situations and tasks. Despite studies investigating the connection between general cognitive functions (such as working memory, attention, executive functions) and statistical learning (Conway, 2020; Frost, Armstrong, \& Christiansen, 2019; Janacsek \& Nemeth, 2013, 2014), an in-depth theory about their relationship is still missing. The competition framework can open up new research lines to discover the dynamic

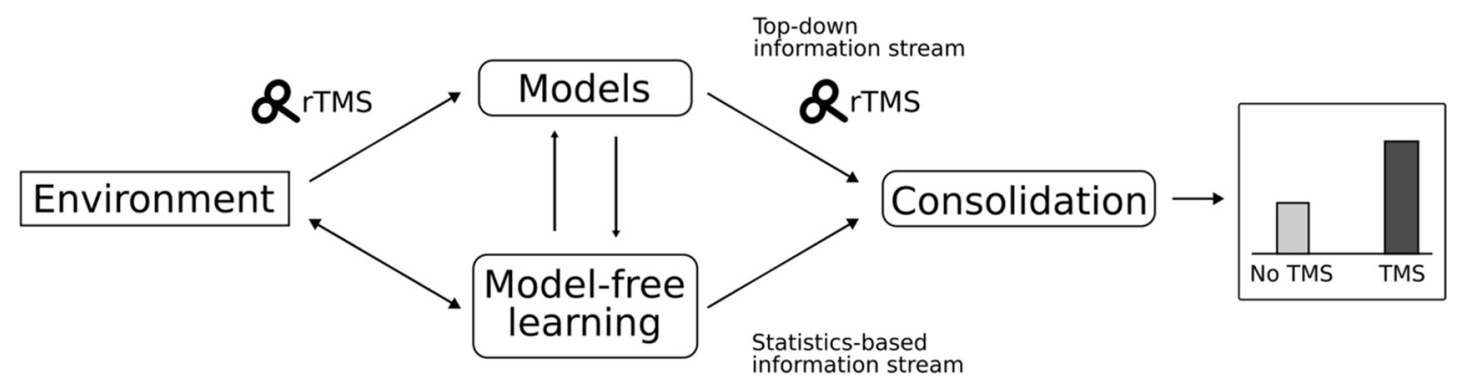

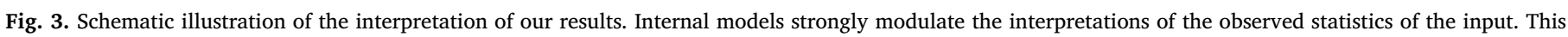

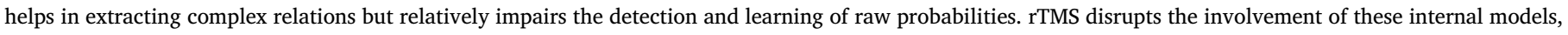
leading to a better consolidation of the newly detected non-adjacent dependencies. 
interactions between general cognitive functions and statistical learning. Future studies should test the competition framework in different statistical learning tasks as well by manipulating the DLPFC.

Our perceptual-motor task with non-adjacent regularities may share similarities with language processes. For example, Nemeth et al. (2011) revealed a relationship between sentence processing and the perceptual-motor statistical learning task used in our study. Here, we found DLPFC-involvement with a non-linguistic perceptual-motor task, and the aftereffects of DLPFC stimulation pointed in the same direction as it was found for a language-related sequence learning task (Smalle et al., 2017). If we broaden the view towards a developmental aspect, language learning and the acquisition of non-adjacent statistical learning appear to share their developmental characteristics: children seem to show better performance both in language learning (Goldowsky \& Newport, 1993; Newport, 1990) and perceptual-motor non-adjacent statistical learning (Janacsek et al., 2012; Zwart et al., 2019). Based on the late maturation of the DLPFC (Bunge \& Zelazo, 2006; Gogtay et al., 2004; Kadosh et al., 2014; Thompson et al., 2001), we can speculate that the mechanism behind the children's superiority in these two skills may be related to the effect of TMS over the DLPFC: not having built rigid models about our environment may help liberate our model-free approaches to support the learning of new skills. This notion is also in line with the finding that the model-based strategy is absent in childhood and gradually strengthens during adolescence up to adulthood (Decker, Otto, Daw, \& Hartley, 2016). Future studies directly examining the connection between model-free and model-based processes in language tasks, ideally from a developmental aspect, should be conducted.

Several studies have used non-invasive brain stimulation over Broca's area to investigate adjacent and non-adjacent dependencies in artificial grammar learning tasks (De Vries et al., 2010; Uddén et al., 2008; Uddén, Ingvar, Hagoort, \& Petersson, 2017). Using the same $1 \mathrm{~Hz}$ TMS stimulation protocol, they found weaker non-adjacent but better adjacent learning. Therefore, we suggest that the ventral areas (e.g., Broca's area) might have a different role in the acquisition of non-adjacent dependencies than the dorsal part of the lateral frontal cortex (e.g., Brodmann 9 area, targeted in our study). Yet in natural language, multiple simultaneous non-adjacent dependencies are present (De Vries, Christiansen, \& Petersson, 2011), which makes the comparison between the effects on second-order dependencies used in the present study and language learning more complicated. Further research is warranted to reveal the different roles of the dorsal vs. ventral parts of the lateral frontal areas in linguistic learning processes and non-linguistic statistical learning.

Previous studies revealed a possible methodological issue: the interhemispheric compensation might obscure the effects following unilateral stimulation. This effect might have played a role in the negative results of Savic et al. (2017). Galea et al. (2010) found the left hemisphere advantage, but it does not indicate that the activation of the right hemisphere cannot interfere with the results. TMS studies proved that lateralization does not necessarily suggest that the function is eliminated from the other hemisphere, even in the case of language processing (e.g., Hartwigsen et al., 2010) or working memory (e.g., Mottaghy, Döring, Müller-Gärtner, Töpper, \& Krause, 2002; Vékony et al., 2018). These results indicate that even if the dominating hemisphere is stimulated, the other can have confounding effects on the results. Thus, we eliminated this possible confounding factor using bilateral brain stimulation to disrupt the involvement of both DLPFCs during learning. Future studies could benefit from using both unilateral and bilateral stimulation in one experimental design to get a holistic picture of the role of the DLPFC in statistical learning.

Finally, there are some limitations to our study. First, we only assessed the level of TMS discomfort after the $24 \mathrm{~h}$ retest session. Thus, the relatively long elapsed time between the stimulation and assessment could have influenced the precision of the subjects' ratings. The reason for this was that we did not intend to draw the participants' attention to the perceived (lack of) TMS discomfort. It would have created a belief in the participants about their allocation to the DLPFC or Sham Group, which could have biased our results unnecessarily. Another limitation of our study is that we chose sham stimulation as a control condition: participants were stimulated with a perpendicularly oriented TMS coil. Using such sham control conditions, one can test whether the stimulation of the target area modifies specific processes. However, the regional specificity (i.e., whether stimulation over other regions would not lead to similar changes in performance) can be claimed only with an active control condition (Duecker \& Sack, 2015). In our study, the average RTs and accuracy were not altered by the stimulation, suggesting that our results are not due to the modulation of general arousal or attention (Kosinski, 2008), but instead due to the involvement of the targeted DLPFC area in statistical learning itself. Notably, active control stimulation instead of sham stimulation, as suggested above, may also not be optimal as it does not control for sensory side effects (Duecker \& Sack, 2015). Therefore, in future studies, it would be beneficial to utilize both types of controls within the same experimental design to reveal the regional specificity of the DLPFC for boosting statistical learning.

To sum up, we observed that the bilateral disruption of the DLPFCs during the training session had a beneficial effect on non-adjacent statistical learning performance that was observable after a 24-hour offline period. Our findings are significant in three aspects. First, this finding provides mechanistic level evidence for the models positing an antagonistic relationship between the model-based and model-free processes. Second, from a methodological viewpoint, previous investigations using external non-invasive brain stimulation methods stimulated only one hemisphere at a time. Therefore, the taking-over of the lost function by the contralateral hemisphere cannot be ruled out in earlier studies (Janacsek et al., 2015; Savic et al., 2017). Here, we showed that the sequential application of $1 \mathrm{~Hz}$ rTMS before learning blocks over both hemispheres establishes and sustains the inhibitory effect. The finding that no effect of stimulation order was observed supports the viability and practicality of this approach. It may form the basis for future research requiring bi-hemispherical/multi-site intervention. Third, and most importantly, our results raise a new possible theoretical framework explaining the role of the DLPFC in statistical learning and consolidation processes. Our findings shed light on the importance of exploring the possible interactive mechanisms underlying learning. This approach can help us more deeply understand the exact mechanism of skill acquisition and consolidation, and create a bridge between the research fields of general cognitive functions and statistical learning.

\section{Declaration of Competing Interest}

The authors declare that they have no known competing financial interests or personal relationships that could have appeared to influence the work reported in this paper.

\section{Acknowledgments}

This work was supported by a Deutsche Forschungsgemeinschaft Grant (KO3918/5-1, PI: G.K.) and also by the National Brain Research Program (project 2017-1.2.1-NKP-2017-00002); Hungarian Scientific Research Fund (NKFIH-OTKA K 128016, PI: D. N., NKFIH-OTKA PD 124148, PI: K.J.); Janos Bolyai Research Fellowship of the Hungarian Academy of Sciences (to K. J.); IDEXLYON Fellowship of the University of Lyon as part of the Programme Investissements d'Avenir (ANR-16IDEX-0005) (to D.N). The authors would like to thank Mareike Grotheer and Catarina Amado for performing the MRI scans, Maria Dotzer, Fabienne Windel, and Rebecca Mayer for their help in recruiting the participants. 


\section{Appendix A. Supplementary material}

Supplementary data to this article can be found online at https:// doi.org/10.1016/j.jml.2020.104144.

\section{References}

Albouy, G., Fogel, S., King, B. R., Laventure, S., Benali, H., Karni, A., ... Doyon, J. (2015). Maintaining vs. enhancing motor sequence memories: Respective roles of striatal and hippocampal systems. NeuroImage, 108, 423-434. https://doi.org/10.1016/j. neuroimage.2014.12.049.

Albouy, G., King, B. R., Maquet, P., \& Doyon, J. (2013). Hippocampus and striatum: Dynamics and interaction during acquisition and sleep-related motor sequence memory consolidation. Hippocampus, 23(11), 985-1004. https://doi.org/10.1002/ hipo. 22183.

Albouy, G., Sterpenich, V., Balteau, E., Vandewalle, G., Desseilles, M., Dang-Vu, T., ... Maquet, P. (2008). Both the hippocampus and striatum are involved in consolidation of motor sequence memory. Neuron, 58(2), 261-272. https://doi.org/10.1016/j. neuron.2008.02.008.

Baier, B., Karnath, H. O., Dieterich, M., Birklein, F., Heinze, C., \& Müller, N. G. (2010) Keeping memory clear and stable - The contribution of human basal ganglia and prefrontal cortex to working memory. Journal of Neuroscience, 30(29), 9788-9792. https://doi.org/10.1523/JNEUROSCI.1513-10.2010.

Beese, C., Meyer, L., Vassileiou, B., \& Friederici, A. D. (2017). Temporally and spatially distinct theta oscillations dissociate a language-specific from a domain-general processing mechanism across the age trajectory. Scientific Reports, 7(1), 1-11. https:// doi.org/10.1038/s41598-017-11632-z.

Beierholm, U. R., Anen, C., Quartz, S., \& Bossaerts, P. (2011). Separate encoding of model-based and model-free valuations in the human brain. NeuroImage, 58(3), 955-962. https://doi.org/10.1016/j.neuroimage.2011.06.071.

Bennett, I. J., Madden, D. J., Vaidya, C. J., Howard, J. H., \& Howard, D. V. (2011). White matter integrity correlates of implicit sequence learning in healthy aging. Neurobiology of Aging, 32(12), 2317.e1-2317.e12. https://doi.org/10.1016/j. neurobiolaging.2010.03.017.

Blumenfeld, R. S., \& Ranganath, C. (2007). Prefrontal cortex and long-term memory encoding: An integrative review of findings from neuropsychology and neuroimaging. Neuroscientist, 13(3), 280-291. https://doi.org/10.1177/1073858407299290.

Buckner, R. L., \& DiNicola, L. M. (2019). The brain's default network: Updated anatomy, physiology and evolving insights. Nature Reviews Neuroscience, 20(10), 593-608. https://doi.org/10.1038/s41583-019-0212-7.

Bunge, S. A., \& Zelazo, P. D. (2006). A brain-based account of the development of rule use in childhood. Current Directions in Psychological Science, 15(3), 118-121. https://doi. org/10.1111/j.0963-7214.2006.00419.x.

Case, R., Kurland, D. M., \& Goldberg, J. (1982). Operational efficiency and the growth of short-term memory span. Journal of Experimental Child Psychology, 33(3), 386-404. https://doi.org/10.1016/0022-0965(82)90054-6.

Christiansen, M. H., \& Chater, N. (2015). The language faculty that wasn't: A usage-based account of natural language recursion. Frontiers in Psychology, 6, 1182. https://doi. org/10.3389/fpsyg.2015.01182.

Cieslik, E. C., Zilles, K., Caspers, S., Roski, C., Kellermann, T. S., Jakobs, O., ... Eickhoff, S. B. (2013). Is there one DLPFC in cognitive action control? Evidence for heterogeneity from Co-activation-based parcellation. Cerebral Cortex, 23(11), 2677-2689. https:// doi.org/10.1093/cercor/bhs256.

Conway, A. R. A., Kane, M. J., Bunting, M. F., Hambrick, D. Z., Wilhelm, O., \& Engle, R. W. (2005). Working memory span tasks: A methodological review and user's guide. Psychonomic Bulletin and Review, 12(5), 769-786. https://doi.org/10.3758/ BF03196772.

Conway, C. M. (2020). How does the brain learn environmental structure? Ten core principles for understanding the neurocognitive mechanisms of statistical learning. Neuroscience and Biobehavioral Reviews, 112, 279-299. https://doi.org/10.1016/j. neubiorev.2020.01.032.

Culbreth, A. J., Westbrook, A., Daw, N. D., Botvinick, M., \& Barch, D. M. (2016). Reduced model-based decision-making in schizophrenia. Journal of Abnormal Psychology, 125(6), 777-787. https://doi.org/10.1037/abn0000164.

Daw, N. D., Gershman, S. J., Seymour, B., Dayan, P., \& Dolan, R. J. (2011). Model-based influences on humans' choices and striatal prediction errors. Neuron, 69(6), 1204-1215. https://doi.org/10.1016/j.neuron.2011.02.027.

Daw, N. D., Niv, Y., \& Dayan, P. (2005). Uncertainty-based competition between prefrontal and dorsolateral striatal systems for behavioral control. Nature Neuroscience, 8(12), 1704-1711. https://doi.org/10.1038/nn1560.

Dayan, P., \& Berridge, K. C. (2014). Model-based and model-free Pavlovian reward learning: Revaluation, revision, and revelation. Cognitive, Affective and Behavioral Neuroscience, 14(2), 473-492. https://doi.org/10.3758/s13415-014-0277-8.

De Vries, M. H., Barth, A. C. R., Maiworm, S., Knecht, S., Zwitserlood, P., \& Flöel, A. (2010). Electrical stimulation of Broca's area enhances implicit learning of an artificial grammar. Journal of Cognitive Neuroscience, 22(11), 2427-2436. https://doi. org/10.1162/jocn.2009.21385.

De Vries, M. H., Christiansen, M. H., \& Petersson, K. M. (2011). Learning recursion: Multiple nested and crossed dependencies. Biolinguistics, 5(1-2), 10-35. http://www. biolinguistics.eu.

Decker, J. H., Otto, A. R., Daw, N. D., \& Hartley, C. A. (2016). From creatures of habit to goal-directed learners: Tracking the developmental emergence of model-based reinforcement learning. Psychological Science, 27(6), 848-858. https://doi.org/10. $1177 / 0956797616639301$.
Duecker, F., \& Sack, A. T. (2015). Rethinking the role of sham TMS. Frontiers in Psychology, 6, 210. https://doi.org/10.3389/fpsyg.2015.00210.

Düzel, E., Penny, W. D., \& Burgess, N. (2010). Brain oscillations and memory. Current Opinion in Neurobiology, 20(2), 245-257. https://doi.org/10.1016/j.conb.2010.01. 004.

Engle, R. W., Laughlin, J. E., Tuholski, S. W., \& Conway, A. R. A. (1999). Working memory, short-term memory, and general fluid intelligence: A latent-variable approach. Journal of Experimental Psychology: General, 128(3), 309-331. https://doi. org/10.1037/0096-3445.128.3.309.

Figner, B., Knoch, D., Johnson, E. J., Krosch, A. R., Lisanby, S. H., Fehr, E., \& Weber, E. U. (2010). Lateral prefrontal cortex and self-control in intertemporal choice. Nature Neuroscience, 13(5), 538-539. https://doi.org/10.1038/nn.2516.

Filoteo, J. V., Lauritzen, S., \& Maddox, W. T. (2010). Removing the frontal lobes: The effects of engaging executive functions on perceptual category learning. Psychological Science, 21(3), 415-423. https://doi.org/10.1177/0956797610362646.

Fingelkurts, A. A., Fingelkurts, A. A., Kallio, S., \& Revonsuo, A. (2007). Cortex functional connectivity as a neurophysiological correlate of hypnosis: An EEG case study. Neuropsychologia, 45(7), 1452-1462. https://doi.org/10.1016/j.neuropsychologia. 2006.11.018.

Fiser, J., Berkes, P., Orbán, G., \& Lengyel, M. (2010). Statistically optimal perception and learning: From behavior to neural representations. Trends in Cognitive Sciences, 14, 119-130. https://doi.org/10.1016/j.tics.2010.01.003.

Fox, C. J., Mueller, S. T., Gray, H. M., Raber, J., \& Piper, B. J. (2013). Evaluation of a short-form of the Berg Card Sorting Test. PLoS ONE, 8(5), e63885. https://doi.org/ 10.1371/journal.pone.0063885.

Frost, R., Armstrong, B. C., \& Christiansen, M. H. (2019). Statistical learning research: A critical review and possible new directions. Psychological Bulletin, 145(12), 1128-1153. https://doi.org/10.1037/bul0000210.

Galea, J. M., Albert, N. B., Ditye, T., \& Miall, R. C. (2010). Disruption of the dorsolateral prefrontal cortex facilitates the consolidation of procedural skills. Journal of Cognitive Neuroscience, 22(6), 1158-1164. https://doi.org/10.1162/jocn.2009.21259.

Gogtay, N., Giedd, J. N., Lusk, L., Hayashi, K. M., Greenstein, D., Vaituzis, A. C., .. Thompson, P. M. (2004). Dynamic mapping of human cortical development during childhood through early adulthood. Proceedings of the National Academy of Sciences of the United States of America, 101(21), 8174-8179. https://doi.org/10.1073/pnas. 0402680101.

Goldowsky, B. N., \& Newport, E. L. (1993). The proceedings of the twenty-fourth annual Child Language Research Forum. In The proceedings of the twenty-fourth annual child language research forum (p. 124). Center for the Study of Language (CSLI).

Groiss, S., Ugawa, Y., Paulus, W., \& Huang, Y.-Z. (2012). Patterned protocols of transcranial magnetic stimulation. In C. Miniussi, W. Paulus, \& P. M. Rossini (Eds.), Transcranial Brain Stimulation (pp. 135-158). 10.1201/b14174-8.

Haith, A. M., \& Krakauer, J. W. (2013). Model-based and model-free mechanisms of human motor learning. Advances in Experimental Medicine and Biology, 782, 1-21. https://doi.org/10.1007/978-1-4614-5465-6_1.

Hartwigsen, G., Baumgaertner, A., Price, C. J., Koehnke, M., Ulmer, S., \& Siebner, H. R. (2010). Phonological decisions require both the left and right supramarginal gyri. Proceedings of the National Academy of Sciences of the United States of America, 107(38), 16494-16499. https://doi.org/10.1073/pnas.1008121107.

Howard, J. H., \& Howard, D. V. (1997). Age differences in implicit learning of higher order dependencies in serial patterns. Psychology and Aging, 12(4), 634-656. https:// doi.org/10.1037/0882-7974.12.4.634.

Howard, D. V., Howard, J. H., Japikse, K., DiYanni, C., Thompson, A., \& Somberg, R. (2004). Implicit sequence learning: Effects of level of structure, adult age, and extended practice. Psychology and Aging, 19(1), 79-92. https://doi.org/10.1037/08827974.19.1.79.

Janacsek, K., Ambrus, G. G., Paulus, W., Antal, A., \& Nemeth, D. (2015). Right hemisphere advantage in statistical learning: Evidence from a probabilistic sequence learning task. Brain Stimulation, 8(2), 277-282. https://doi.org/10.1016/j.brs.2014. 11.008 .

Janacsek, K., Fiser, J., \& Nemeth, D. (2012). The best time to acquire new skills: Agerelated differences in implicit sequence learning across the human lifespan. Developmental Science, 15(4), 496-505. https://doi.org/10.1111/j.1467-7687.2012. 01150.x.

Janacsek, K., \& Nemeth, D. (2013). Implicit sequence learning and working memory: Correlated or complicated? Cortex, 49(8), 2001-2006. https://doi.org/10.1016/j. cortex.2013.02.012.

Janacsek, K., \& Nemeth, D. (2014). The puzzle is complicated: when should working memory be related to implicit sequence learning, and when should it not? (Response to Martini et al.). Cortex, 64(411-412), https://doi.org/10.1016/j.cortex.2014.07. 020 .

Juhasz, D., Nemeth, D., \& Janacsek, K. (2019). Is there more room to improve? The lifespan trajectory of procedural learning and its relationship to the between- and within-group differences in average response times. PLOS ONE, 14(7), 593582. https://doi.org/10.1371/journal.pone.0215116.

Kadosh, K. C., Heathcote, L. C., \& Lau, J. Y. F. (2014). Age-related changes in attentional control across adolescence: How does this impact emotion regulation capacities? Frontiers in Psychology, 5, 111. https://doi.org/10.3389/fpsyg.2014.00111.

Kaufman, S. B., DeYoung, C. G., Gray, J. R., Jiménez, L., Brown, J., \& Mackintosh, N. (2010). Implicit learning as an ability. Cognition, 116(3), 321-340. https://doi.org/ 10.1016/j.cognition.2010.05.011.

Klomjai, W., Katz, R., \& Lackmy-Vallée, A. (2015). Basic principles of transcranial magnetic stimulation (TMS) and repetitive TMS (rTMS). Annals of Physical and Rehabilitation Medicine, 58(4), 208-213. https://doi.org/10.1016/j.rehab.2015.05. 005.

Kóbor, A., Horváth, K., Kardos, Z., Nemeth, D., \& Janacsek, K. (2019). Perceiving 
structure in unstructured stimuli: Implicitly acquired prior knowledge impacts the processing of unpredictable transitional probabilities. BioRxiv, 738419. https://doi. org/10.1101/738419.

Kóbor, A., Janacsek, K., Takács, A., \& Nemeth, D. (2017). Statistical learning leads to persistent memory: Evidence for one-year consolidation. Scientific Reports, 7(1), 1-10. https://doi.org/10.1038/s41598-017-00807-3.

Koechlin, E., \& Summerfield, C. (2007). An information theoretical approach to prefrontal executive function. Trends in Cognitive Sciences, 11(6), 229-235. https://doi.org/10. 1016/j.tics.2007.04.005.

Kosinski, R. J. (2008). A literature review on reaction time. Clemson University, 10 337-344. http://biology.clemson.edu/bpc/bp/Lab/110/reaction.htm.

Kurth-Nelson, Z., Bickel, W., \& Redish, A. D. (2012). A theoretical account of cognitive effects in delay discounting. European Journal of Neuroscience, 35(7), 1052-1064. https://doi.org/10.1111/j.1460-9568.2012.08058.x.

Lara, A. H., \& Wallis, J. D. (2015). The role of prefrontal cortex in working memory: A mini review. Frontiers in Systems Neuroscience, 9, 173. https://doi.org/10.3389/fnsys. 2015.00173.

Lieberman, M. D. (2000). Intuition: A social cognitive neuroscience approach. Psychological Bulletin, 126(1), 109-137. https://doi.org/10.1037/0033-2909.126.1. 109.

Lisanby, S. H., Gutman, D., Luber, B., Schroeder, C., \& Sackeim, H. A. (2001). Sham TMS: Intracerebral measurement of the induced electrical field and the induction of motorevoked potentials. Biological Psychiatry, 49(5), 460-463. https://doi.org/10.1016/ S0006-3223(00)01110-0.

Matsunaga, K., Maruyama, A., Fujiwara, T., Nakanishi, R., Tsuji, S., \& Rothwell, J. C. (2005). Increased corticospinal excitability after $5 \mathrm{~Hz}$ rTMS over the human supplementary motor area. Journal of Physiology, 562(1), 295-306. https://doi.org/10. 1113/jphysiol.2004.070755.

McNab, F., \& Klingberg, T. (2008). Prefrontal cortex and basal ganglia control access to working memory. Nature Neuroscience, 11(1), 103-107. https://doi.org/10.1038/ nn2024.

Mottaghy, F. M., Döring, T., Müller-Gärtner, H. W., Töpper, R., \& Krause, B. J. (2002). Bilateral parieto-frontal network for verbal working memory: An interference approach using repetitive transcranial magnetic stimulation (rTMS). European Journal of Neuroscience, 16(8), 1627-1632. https://doi.org/10.1046/j.1460-9568.2002. 02209.x.

Nemeth, D., \& Janacsek, K. (2011). The dynamics of implicit skill consolidation in young and elderly adults. Journals of Gerontology - Series B Psychological Sciences and Social Sciences, 66 B(1), 15-22. https://doi.org/10.1093/geronb/gbq063.

Nemeth, D., Janacsek, K., Csifcsak, G., Szvoboda, G., Howard, J. H., \& Howard, D. V. (2011). Interference between sentence processing and probabilistic implicit sequence learning. PLOS ONE, 6(3), https://doi.org/10.1371/journal.pone.0017577.

Nemeth, D., Janacsek, K., \& Fiser, J. (2013). Age-dependent and coordinated shift in performance between implicit and explicit skill learning. Frontiers in Computational Neuroscience, 7, 147. https://doi.org/10.3389/fncom.2013.00147.

Nemeth, D., Janacsek, K., Londe, Z., Ullman, M. T., Howard, D. V., \& Howard, J. H. (2010). Sleep has no critical role in implicit motor sequence learning in young and old adults. Experimental Brain Research, 201(2), 351-358. https://doi.org/10.1007/ s00221-009-2024-X.

Nemeth, D., Janacsek, K., Polner, B., \& Kovacs, Z. A. (2013). Boosting human learning by hypnosis. Cerebral Cortex, 23(4), 801-805. https://doi.org/10.1093/cercor/bhs068.

Newell, B. R., Moore, C. P., Wills, A. J., \& Milton, F. (2013). Reinstating the frontal lobes? Having more time to think improves implicit perceptual categorization: A Comment on Filoteo, Lauritzen, and Maddox (2010). Psychological Science, 24(3), 386-389. https://doi.org/10.1177/0956797612457387.

Newport, E. L. (1990). Maturational constraints on language learning. Cognitive Science, 14(1), 11-28. https://doi.org/10.1016/0364-0213(90)90024-Q.

Oakley, D. A., \& Halligan, P. W. (2009). Hypnotic suggestion and cognitive neuroscience. Trends in Cognitive Sciences, 13(6), 264-270. https://doi.org/10.1016/j.tics.2009.03. 004.

Otto, A. R., Raio, C. M., Chiang, A., Phelps, E. A., \& Daw, N. D. (2013). Working-memory capacity protects model-based learning from stress. Proceedings of the National Academy of Sciences of the United States of America, 110(52), 20941-20946. https:// doi.org/10.1073/pnas.1312011110.

Otto, A. R., Skatova, A., Madlon-Kay, S., \& Daw, N. D. (2014). Cognitive control predicts use of model-based reinforcement learning. Journal of Cognitive Neuroscience, 27(2), 319-333. https://doi.org/10.1162/jocn_a_00709.

Packard, M. G., \& Knowlton, B. J. (2002). Learning and memory functions of the basal ganglia. Annual Review of Neuroscience, 25(1), 563-593. https://doi.org/10.1146/ annurev.neuro.25.112701.142937.

Pascual-Leone, A., Wassermann, E. M., Grafman, J., \& Hallett, M. (1996). The role of the dorsolateral prefrontal cortex in implicit procedural learning. Experimental Brain Research, 107(3), 479-485. https://doi.org/10.1007/BF00230427.

Peinemann, A., Reimer, B., Löer, C., Quartarone, A., Münchau, A., Conrad, B., \& Siebner, H. R. (2004). Long-lasting increase in corticospinal excitability after 1800 pulses of subthreshold $5 \mathrm{~Hz}$ repetitive TMS to the primary motor cortex. Clinical Neurophysiology, 115(7), 1519-1526. https://doi.org/10.1016/j.clinph.2004.02.005. Remillard, G. (2008). Implicit learning of second-, third-, and fourth-order adjacent and nonadjacent sequential dependencies. Quarterly Journal of Experimental Psychology, 61(3), 400-424. https://doi.org/10.1080/17470210701210999.

Robertson, E. M., Théoret, H., \& Pascual-Leone, A. (2003). Studies in cognition: The problems solved and created by transcranial magnetic stimulation. Journal of Cognitive Neuroscience, 15(7), 948-960. https://doi.org/10.1162/
089892903770007344

Ross, R. S., Brown, T. I., \& Stern, C. E. (2009). The retrieval of learned sequences engages the hippocampus: Evidence from fMRI. Hippocampus, 19(9), 790-799. https://doi. org/10.1002/hipo.20558.

Savic, B., Cazzoli, D., Müri, R., \& Meier, B. (2017). No effects of transcranial DLPFC stimulation on implicit task sequence learning and consolidation. Scientific Reports, 7(1), 9649. https://doi.org/10.1038/s41598-017-10128-0.

Schad, D. J., Jünger, E., Sebold, M., Garbusow, M., Bernhardt, N., Javadi, A. H., ... Huys, Q. J. M. (2014). Processing speed enhances model-based over model-free reinforcement learning in the presence of high working memory functioning. Frontiers in Psychology, 5, 1450. https://doi.org/10.3389/fpsyg.2014.01450.

Silvanto, J., Cattaneo, Z., Battelli, L., \& Pascual-Leone, A. (2008). Baseline cortical excitability determines whether TMS disrupts or facilitates behavior. Journal of Neurophysiology, 99(5), 2725-2730. https://doi.org/10.1152/jn.01392.2007.

Simon, J. R., Vaidya, C. J., Howard, J. H., \& Howard, D. V. (2012). The effects of aging on the neural basis of implicit associative learning in a probabilistic triplets learning task. Journal of Cognitive Neuroscience, 24(2), 451-463. https://doi.org/10.1162/ jocn_a_00116.

Smalle, E. H. M., Panouilleres, M., Szmalec, A., \& Möttönen, R. (2017). Language learning in the adult brain: Disrupting the dorsolateral prefrontal cortex facilitates word-form learning. Scientific Reports, 7(1), 13966. https://doi.org/10.1038/s41598-017 14547-x.

Smittenaar, P., FitzGerald, T. H. B., Romei, V., Wright, N. D., \& Dolan, R. J. (2013) Disruption of dorsolateral prefrontal cortex decreases model-based in favor of modelfree control in humans. Neuron, 80(4), 914-919. https://doi.org/10.1016/j.neuron. 2013.08.009.

Song, S., Howard, J. H., \& Howard, D. V. (2007). Sleep does not benefit probabilistic motor sequence learning. Journal of Neuroscience, 27(46), 12475-12483. https://doi. org/10.1523/JNEUROSCI.2062-07.2007.

Stillman, C. M., Gordon, E. M., Simon, J. R., Vaidya, C. J., Howard, D. V., \& Howard, J. H. (2013). Caudate resting connectivity predicts implicit probabilistic sequence learning. Brain Connectivity, 3(6), 601-610. https://doi.org/10.1089/brain.2013. 0169.

Thompson, P. M., Vidal, C., Giedd, J. N., Gochman, P., Blumenthal, J., Nicolson, R., .. Rapoport, J. L. (2001). Mapping adolescent brain change reveals dynamic wave of accelerated gray matter loss in very early-onset schizophrenia. Proceedings of the National Academy of Sciences of the United States of America, 98(20), 11650-11655. https://doi.org/10.1073/pnas.201243998.

Thut, G., \& Pascual-Leone, A. (2010). A review of combined TMS-EEG studies to characterize lasting effects of repetitive TMS and assess their usefulness in cognitive and clinical neuroscience. Brain Topography, 22(4), 219-232. https://doi.org/10.1007/ s10548-009-0115-4.

Tóth, B., Janacsek, K., Takács, Á., Kóbor, A., Zavecz, Z., \& Nemeth, D. (2017). Dynamics of EEG functional connectivity during statistical learning. Neurobiology of Learning and Memory, 144, 216-229. https://doi.org/10.1016/j.nlm.2017.07.015.

Uddén, J., Folia, V., Forkstam, C., Ingvar, M., Fernandez, G., Overeem, S., ... Petersson, K. M. (2008). The inferior frontal cortex in artificial syntax processing: An rTMS study. Brain Research, 1224, 69-78. https://doi.org/10.1016/j.brainres.2008.05.070.

Uddén, J., Ingvar, M., Hagoort, P., \& Petersson, K. M. (2017). Broca's region: A causal role in implicit processing of grammars with crossed non-adjacent dependencies. Cognition, 164, 188-198. https://doi.org/10.1016/j.cognition.2017.03.010.

Vékony, T., Marossy, H., Must, A., Vécsei, L., Janacsek, K., \& Nemeth, D. (2020). Skil learning can be independent of speed and accuracy instructions. BioRxiv, 726315. https://doi.org/10.1101/726315.

Vékony, T., Németh, V. L., Holczer, A., Kocsis, K., Kincses, Z. T., Vécsei, L., \& Must, A (2018). Continuous theta-burst stimulation over the dorsolateral prefrontal cortex inhibits improvement on a working memory task. Scientific Reports, 8(1), 14835. https://doi.org/10.1038/s41598-018-33187-3.

Vikbladh, O. M., Meager, M. R., King, J., Blackmon, K., Devinsky, O., Shohamy, D., ... Daw, N. D. (2019). Hippocampal contributions to model-based planning and spatial memory. Neuron, 102(3), 683-693.e4. https://doi.org/10.1016/j.neuron.2019.02. 014.

Virag, M., Janacsek, K., Horvath, A., Bujdoso, Z., Fabo, D., \& Nemeth, D. (2015). Competition between frontal lobe functions and implicit sequence learning: Evidence from the long-term effects of alcohol. Experimental Brain Research, 233(7), 2081-2089. https://doi.org/10.1007/s00221-015-4279-8.

Walsh, V., \& Cowey, A. (2000). Transcranial magnetic stimulation and cognitive neuroscience. Nature Reviews Neuroscience, 1(1), 73-80. https://doi.org/10.1038/ 35036239.

Wan Lee, S., Shimojo, S., \& O’Doherty, J. P. (2014). Neural computations underlying arbitration between model-based and model-free learning. Neuron, 81(3), 687-699. https://doi.org/10.1016/j.neuron.2013.11.028.

Wunderlich, K., Smittenaar, P., \& Dolan, R. J. (2012). Dopamine enhances model-based over model-free choice behavior. Neuron, 75(3), 418-424. https://doi.org/10.1016/j. neuron.2012.03.042.

Yin, H. H., Knowlton, B. J., \& Balleine, B. W. (2004). Lesions of dorsolateral striatum preserve outcome expectancy but disrupt habit formation in instrumental learning. European Journal of Neuroscience, 19(1), 181-189. https://doi.org/10.1111/j.14609568.2004.03095.x.

Zwart, F. S., Vissers, C. T. W. M., Kessels, R. P. C., \& Maes, J. H. R. (2019). Procedural learning across the lifespan: A systematic review with implications for atypical development. Journal of Neuropsychology, 13, 149-182. https://doi.org/10.1111/jnp. 12139. 


\section{Supplementary Materials}

\section{When less is more: Enhanced statistical learning of non-adjacent dependencies after disruption of bilateral DLPFC}

Géza Gergely Ambrus ${ }^{1 *}$, Teodóra Vékony ${ }^{2 *}$, Karolina Janacsek ${ }^{3,4,5^{*}}$, Anna B. C. Trimborn ${ }^{6 *}$, Gyula Kovács ${ }^{1 *}$, Dezso Nemeth ${ }^{3,4,7^{*}}$

(1) Institute of Psychology, Friedrich Schiller University Jena, Leutragraben 1, D-07743 Jena, Germany.

(2) Department of Neurology, University of Szeged, Semmelweis utca 6., H-6725, Szeged, Hungary.

(3) Institute of Psychology, Eötvös Loránd University, Izabella utca 46., H-1064, Budapest, Hungary.

(4) Brain, Memory and Language Research Group, Institute of Cognitive Neuroscience and Psychology, Research Centre for Natural Sciences, Hungarian Academy of Sciences, Magyar tudósok körútja 2., H1117, Budapest, Hungary.

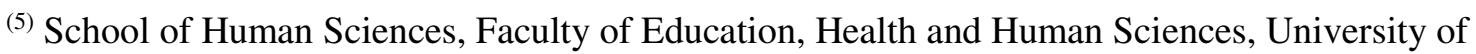
Greenwich, Old Royal Naval College, Park Row, SE10 9LS, London, United Kingdom

${ }^{(6)}$ Donders Institute for Brain, Cognition and Behaviour, Radboud University, Montessorilaan 3, 6525 HR Nijmegen, The Netherlands.

${ }^{(7)}$ Lyon Neuroscience Research Center (CRNL), INSERM, CNRS, Université Claude Bernard Lyon 1, 95 Boulevard Pinel, 69675 Bron, France.

*These authors contributed equally to this work.

Corresponding author: Dezso Nemeth, Lyon Neuroscience Research Center, Université Claude Bernard Lyon 1, 95 Boulevard Pinel, 69675 Bron, France. Email: dezso.nemeth@univlyon1.fr, tel +33766807005

Conflict of interest: The authors report no conflict of interest. 


\section{Analysis of performance separately for high- and low-probability triplets}

A mixed-design ANOVA with the within-subject factors of Session (Training/rTMS session vs. 10 min retest session vs. 2 h retest session vs. 24 h retest session) and Triplet (high- vs. lowprobability) and with the between-subject factor of Group (DLPFC Stimulation vs. Sham Stimulation) was performed. The main effect of Session was significant, $F_{(3,87)}=37.87, p<.001$, $\eta_{p}{ }^{2}=.57$. Note that as here the raw reaction times (RTs) were used as dependent variables, the main effect of Session indicates a change in the average RTs throughout the four sessions. The subsequent post-hoc analysis showed that the RTs in the Training/rTMS session were significantly larger than in the 10 min retest session $(p<.001)$. In the $2 \mathrm{~h}$ retest session, RTs were larger than in the 10 min retest session $(p<.001)$. However, for the $24 \mathrm{~h}$ retest session, the $\mathrm{RT}$ secame shorter compared to the $2 \mathrm{~h}$ retest session $(p<.001)$. No main effect of Group was detected, $F_{(1,29)}=0.78$, $p=.38, \eta_{p}{ }^{2}=.03$, suggesting that the lack of statistically significant RT difference between groups. We did not find group difference in the pattern of change in average RTs throughout the sessions either (revealed by a non-significant Session $\times$ Group interaction: $F_{3,87}=0.45, p=.72, \eta_{p}{ }^{2}=.02$ ).

The main effect of the Triplet factor was significant $F_{1,29}=190.27, p<.001, \eta_{p}{ }^{2}=.87$, indicating that the RTs for the high-probability triplets were faster than for the low-probability triplets. The interaction between the Triplet and Group did not reach significance $F_{1,29}=0.75, p=$ $.39, \eta_{p}{ }^{2}=.03$. However, the interaction of the Triplet and Session factor was significant $F_{3,87}=$ $6.52, p<.001, \eta_{p}{ }^{2}=.18$, indicating that the RTs for high- and low-probability triplets changed diversely on the different levels of Session. The pairwise comparisons showed that for the highprobability triplets, the RTs did change between each session except between the $10 \mathrm{~min}$ and $24 \mathrm{~h}$ retest sessions $(p=.17$, all other $p<.01)$. For the low-probability triplets, the RTs did not change between the Training/rTMS session and $2 \mathrm{~h}$ retest session $(p>.99)$ and between the 10 min retest session and $24 \mathrm{~h}$ retest session $(p=.59)$. Triplet learning was influenced by the stimulation as revealed by a significant Session $\times$ Triplet $\times$ Group interaction $F_{4,116}=4.19, p=.008, \eta_{p}{ }^{2}=.13$. The pairwise comparisons showed that the RTs for the high- and low-probability triplets did differ in both groups, at each time points (all $p<.001$ ), and that RTs did not differ between groups in either session either for high- (all $p>.31$ ) or low-probability triplets (all $p>.25$ ). This indicates that the difference in the dynamics of the learning curve cannot be explained by solely the changes for the high- or low-probability triplets separately. The subsequent two-way ANOVAs revealed that there was a difference between groups at the $24 \mathrm{~h}$ retest session, Triplet $\times$ Group: $F_{1,29}=5.73$, 
$p=.02, \eta_{p}{ }^{2}=.17$, but not at the initial learning session $(p=.86)$, the 10 min retest session $(p=.25)$ or at the $2 \mathrm{~h}$ retest session $(p=.63)$ (Supplementary Figure 1).
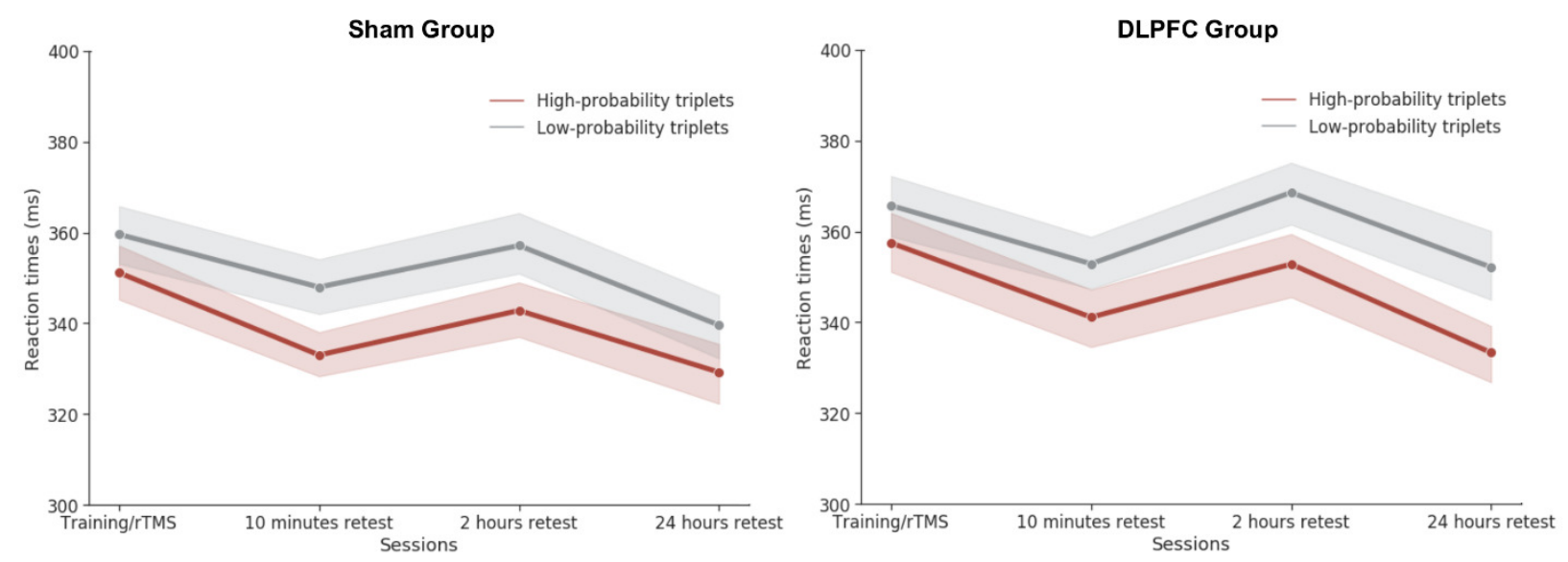

Supplementary Figure 1. The learning performance in the four experimental sessions. The vertical axis indicates the RTs for high- and low-probability triplets, while the horizontal axis presents the four experimental sessions. The red line indicates the RT's for the high-probability triplets, while the gray line the RTs for the low-probability ones. The error bars denote SEM.

\section{Analysis of the Training/rTMS session}

\section{1) Learning index}

We also performed an analysis of the Training/rTMS session with the percentage scores. We ran a mixed-design ANOVA with the within-subject factor of Epoch (Epoch 1-5), and the betweensubject factor of Group (DLPFC vs. Sham). The main effect of Epoch was found to be significant, $F_{4,116}=9.47, p<.001, \eta_{p}{ }^{2}=.25$. Compared to the first epoch, the degree of learning did not change for the second epoch $(p=.73)$; however, in the fourth and the fifth epoch, there were significantly higher learning (first epoch vs. fourth epoch: $p=.001$, first epoch vs. fifth epoch: $p<.001$ ). The interaction of the Epoch and the Group factors were not significant, $F_{4,116}=.41, p=.80, \eta_{p}{ }^{2}=.01$, suggesting that the stimulation did not modify how the learning indices changes during the initial learning phase. The main effect of Group was also not significant $F_{1,29}=0.04, p=.84, \eta_{p}^{2}<.001$, indicating that the overall learning in the initial learning indices in this session was not affected by the stimulation (Supplementary Figure 2). 


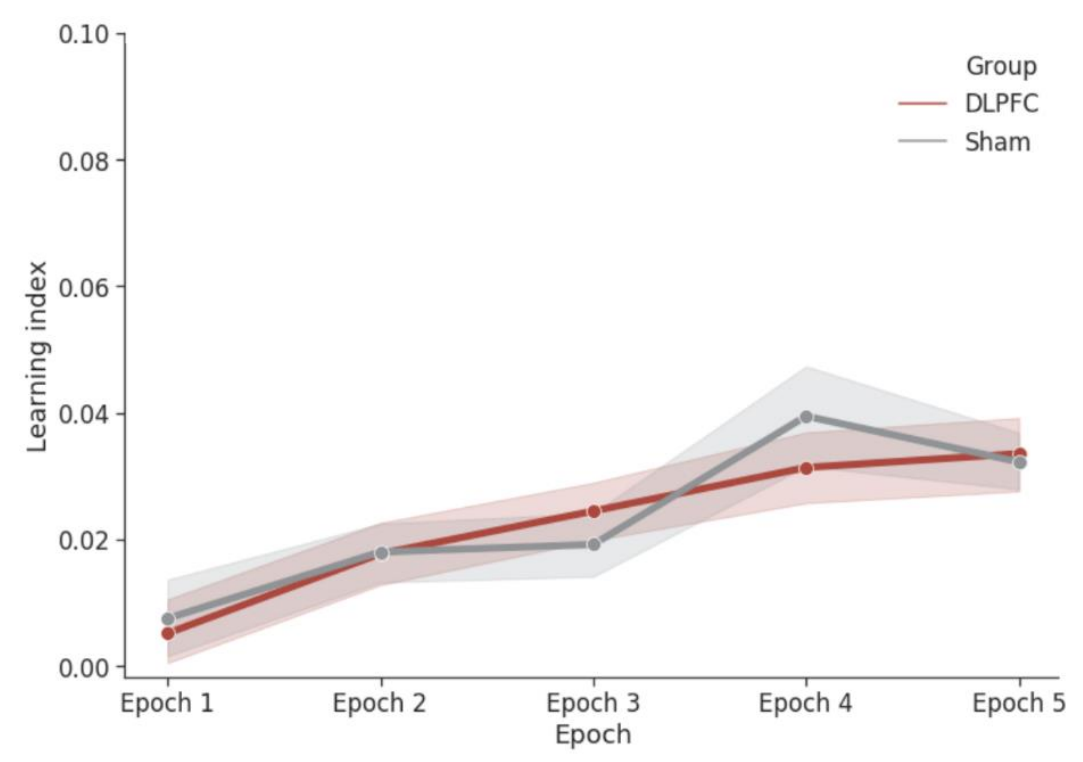

Supplementary Figure 2. The learning indices of Epoch 1 - Epoch 5 in Session 1. The vertical axis indicates the learning indices calculated as [(RTs for low-probability triplet - RTs for high-probability triplets)/RT's for low-probability triplets]. The horizontal axis presents the five epochs of the Training/rTMS session. The red line indicates the learning indices for the DLPFC Group, while the grey line for the Sham Group. The error bars denote SEM.

\section{2) Raw RTs}

We performed a similar analysis with the raw RT scores for the high- and low-probability triplets. We ran a mixed-design ANOVA with the within-subject factors of Epoch (Epoch 1-5) and Triplet (high- vs. low-probability) and with the between-subject factor of Group (DLPFC Stimulation vs. Sham Stimulation). The main effect of Epoch was significant, $\mathrm{F}_{4,116}=86.10, p<.001, \eta_{p}{ }^{2}=.75$. As here the raw RTs were used as dependent variables, the main effect of Epoch indicates that there was a change in the average RTs throughout the five epochs. The pairwise comparisons showed that there was a gradual speed-up of RTs throughout the five epochs. The RTs in the second epoch was significantly shorter than in the first epoch $(p<.001)$, the third epoch's RTs were shorter than the second epoch's RTs $(p=.003)$, and the RTs in the fifth was shorter than in the fourth epoch $(p=.03)$. The RTs between the third and the fourth epoch did not change significantly $(p=.99)$. No significant main effect of Group was detected $F_{1,29}=0.45, p=.51, \eta_{p}{ }^{2}$ $=.02$, suggesting that the average RTs did not differ in the two groups. The Epoch $\times$ Group interaction was not significant, $F_{4,116}=0.14, p=.97, \eta_{p}{ }^{2}=.01$, revealing that the pattern of change in RTs was not affected by the stimulation. 
The main effect of Triplet was significant, $F_{1,29}=109.88, p<.001, \eta_{p}{ }^{2}=.79$ : the RTs for the high-probability triplets were faster than for the low-probability ones. The interaction of the Triplet and Epoch factor was significant, $F_{4,116}=8.24, p<.001, \eta_{p}{ }^{2}=.22$. The pairwise comparisons revealed that for the low-probability triplets, there was no change in RTs between the third and the fourth epoch $(p>.99)$ and only a trend was revealed between the second and the third $(p=.07)$ and the fourth and the fifth $(p=.09)$. In contrast, a gradual change was found between almost every consecutive epoch for the high-probability triplets (except between the fourth and the fifth epoch, $p=.45$, and only a trend was revealed between the third and the fourth epoch, $p=.06$ ). The difference between the high- and low-probability triplets did not reach significance in the first epoch $(p=.11)$, but it did for the last four epochs (all $p<.001)$. The interaction between the Triplet and Group did not reach significance, $F_{1,29}=0.02, p=.89, \eta_{p}{ }^{2}=.001$, suggesting that, overall, the degree of learning was not affected by the stimulation. Moreover, the Epoch $\times$ Triplet $\times$ Group interaction was not significant either, $F_{4,116}=0.40, p=.81, \eta_{p}{ }^{2}=.01$, suggesting that the change in the difference between the high- and low-probability triplets was not affected by the stimulation (Supplementary Figure 3).
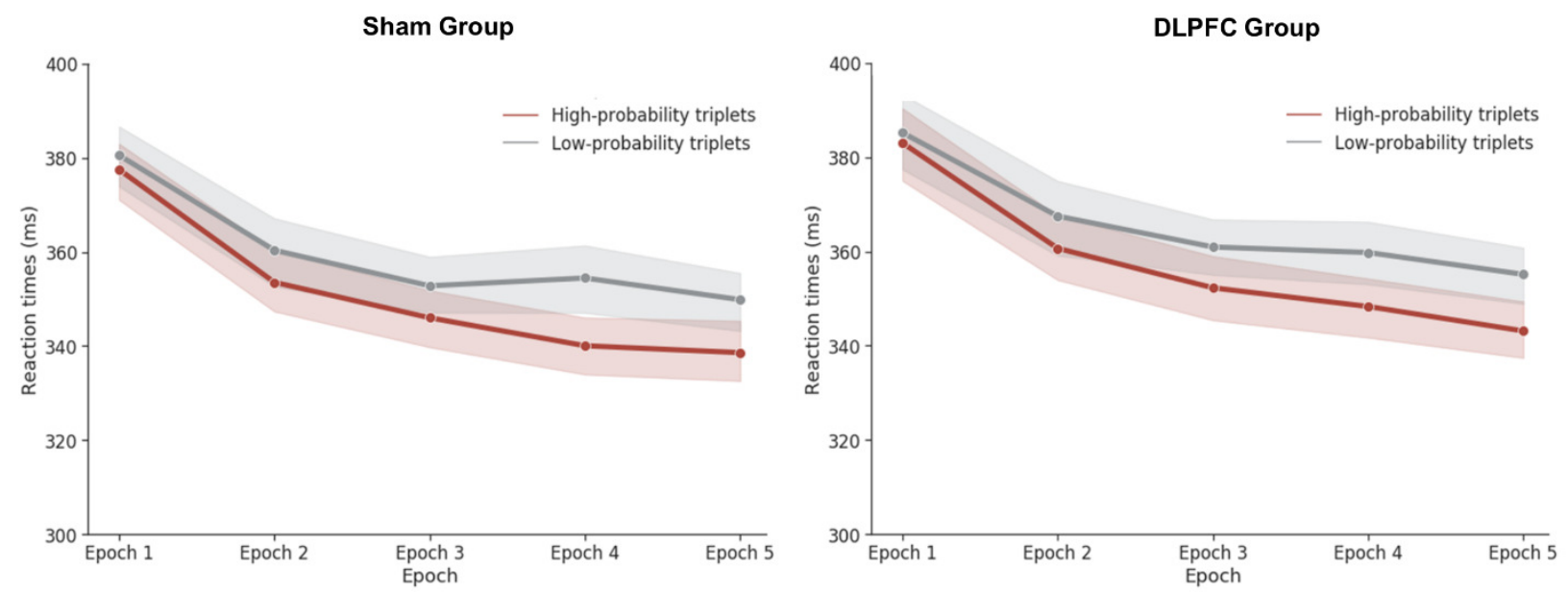

Supplementary Figure 3. The raw RTs of Epoch 1 - Epoch 5 in Session 1. The vertical axis indicates the RTs, while the horizontal axis presents the five epochs of the Training/rTMS session. The red line indicates the RT's for the high-probability triplets, while the gray line the RTs for the low-probability ones. The error bars denote SEM. 


\section{Comparing the performance in the last epoch of the Training/rTMS session to the subsequent sessions}

To check how the performance changed after stimulation has worn off, we performed an analysis of the last epoch of the Training/rTMS session and three subsequent retest session. A mixed-design ANOVA with the within-subject factor of Session $\left(5^{\text {th }}\right.$ Epoch of the Training/rTMS session vs. 10 min retest session vs. 2 h retest session vs. 24 h retest Session) and the between-subject factor of Group (DLPFC Stimulation vs. Sham Stimulation) was run on the percentage learning indices as described above. The main effect of Session was not significant, $F_{3,87}=1.22, p=.31, \eta_{p}{ }^{2}=.04$, indicating that, overall, the degree of learning did not change from the end of the Training/rTMS session. The main effect of Group was also not significant, $F_{1,29}=.66, p=.42, \eta_{p}^{2}=.02$, suggesting that the overall learning score was not affected by the stimulation. Importantly, the interaction of the Session and Group factors was significant, $F_{3,87}=3.29, p=.03, \eta_{p}{ }^{2}=.10$, indicating that the degree of learning in the four sessions was differently affected by the stimulation. The pairwise comparisons showed that in the Sham Group, the learning indices did not change across the sessions (all $p>.60$ ). In contrast, in the DLPFC Group, the learning indices showed a trend-level improvement between the Training/rTMS session and the $24 \mathrm{~h}$ retest session $(p=.10)$, and between the $10 \mathrm{~min}$ retest session and the $24 \mathrm{~h}$ retest session $(p=.08)$. Moreover, learning was higher for the DLPFC Group at $24 \mathrm{~h}$ retest session compared to the Sham Group ( $p$ $=.03$ ), but not at the other three sessions (all $p>.25)$ (Supplementary Figure 4).

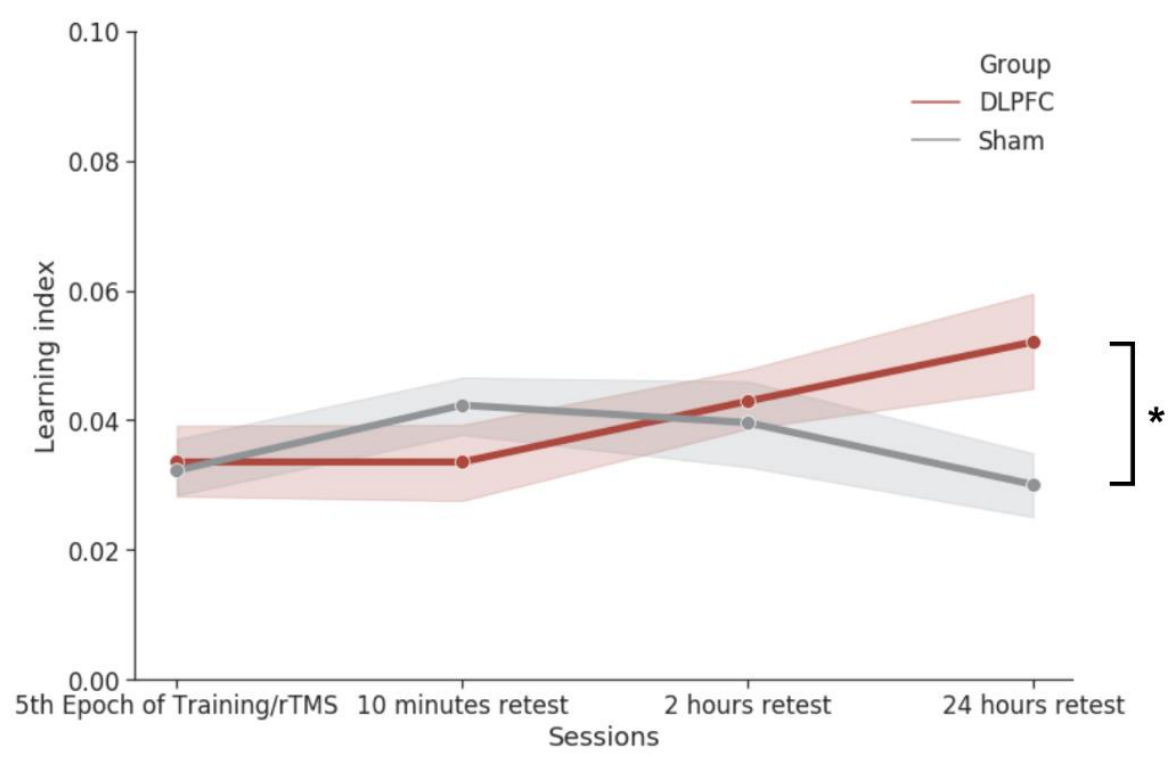


Supplementary Figure 4. The learning indices of the four experimental sessions. The vertical axis indicates the learning indices calculated as [(RTs for low-probability triplet - RTs for high-probability triplets)/RT's for low-probability triplets]. The horizontal axis presents the four experimental sessions (for the Training/rTMS session, only the last epoch is included). The red line indicates the learning indices for the DLPFC Group, while the grey line for the Sham Group. The error bars denote SEM. 


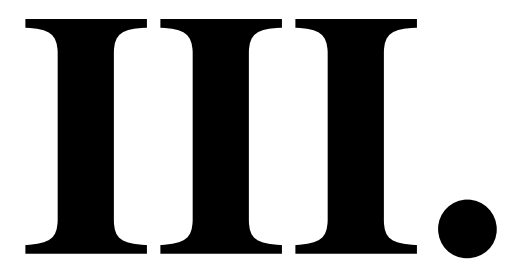




\title{
Speed or Accuracy Instructions During Skill Learning do not Affect the Acquired Knowledge
}

\author{
Teodóra Vékony ${ }^{1}$, Hanna Marossy ${ }^{2}$, Anita Must ${ }^{3}$, László Vécsei ${ }^{1,4}$, \\ Karolina Janacsek (1D) $2,5,6, \dagger$ and Dezso Nemeth (1D) $2,5,7, \dagger$
}

${ }^{1}$ Department of Neurology, University of Szeged, 6725 Szeged, Hungary, ${ }^{2}$ Institute of Psychology, ELTE Eötvös Loránd University, 1064 Budapest, Hungary, ${ }^{3}$ Institute of Psychology, University of Szeged, 6722 Szeged, Hungary, ${ }^{4}$ MTA-SZTE Neuroscience Research Group, University of Szeged, 6725 Szeged, Hungary, ${ }^{5}$ Brain, Memory and Language Research Group, Institute of Cognitive Neuroscience and Psychology, Research Centre for Natural Sciences, 1117 Budapest, Hungary, ${ }^{6}$ Centre for Thinking and Learning, Institute for Lifecourse Development, School of Human Sciences, Faculty of Education, Health and Human Sciences, University of Greenwich, Old Royal Naval College, London, SE10 9LS UK and ${ }^{7}$ Lyon Neuroscience Research Center (CRNL), INSERM, CNRS, Université Claude Bernard Lyon 1, 69675 Bron, France

Address correspondence to Dezso Nemeth, Lyon Neuroscience Research Center (CRNL), INSERM, CNRS, Université de Lyon, Centre Hospitalier Le Vinatier Bâtiment 462 - Neurocampus 95 boulevard Pinel, 69675 Bron, France. E-mail: dezso.nemeth@univ-lyon1.fr.

†These authors contributed equally to this work.

\begin{abstract}
A crucial question in skill learning research is how instruction affects the performance or the underlying representations. Little is known about the effects of instructions on one critical aspect of skill learning, namely, picking-up statistical regularities. More specifically, the present study tests how prelearning speed or accuracy instructions affect the acquisition of non-adjacent second-order dependencies. We trained 2 groups of participants on an implicit probabilistic sequence learning task: one group focused on being fast and the other on being accurate. As expected, we detected a strong instruction effect: accuracy instruction resulted in a nearly errorless performance, and speed instruction caused short reaction times (RTs). Despite the differences in the average RTs and accuracy scores, we found a similar level of statistical learning performance in the training phase. After the training phase, we tested the 2 groups under the same instruction (focusing on both speed and accuracy), and they showed comparable performance, suggesting a similar level of underlying statistical representations. Our findings support that skill learning can result in robust representations, and they highlight that this form of knowledge may appear with almost errorless performance. Moreover, multiple sessions with different instructions enabled the separation of competence from performance.
\end{abstract}

Key words: implicit learning, instruction, probabilistic learning, speed-accuracy, statistical learning 


\section{Introduction}

Our social, motor, and cognitive skills help us adapt to and function in various situations in our everyday life. Therefore, fine-tuning the ability to learn new skills can be advantageous for an individual. Previous studies investigating sports performance (Beilock et al. 2004, 2008) and sequence learning (Hoyndorf and Haider 2009; Barnhoorn et al. 2019) found that speed and accuracy strategies differently affect skill learning. However, skill learning is multifaceted, and it is still not clear what underlying mechanisms benefit from speed and accuracy instructions and what mechanisms do not. A core component of learning new skills is picking up complex statistical regularities from the environment (Janacsek et al. 2012; Conway 2020). To date, no study has investigated the effects of prioritizing speed or accuracy on the acquisition of such statistical dependencies. Here, we aim to unveil how emphasizing speed or accuracy influences this essential aspect of skill learning.

Hoyndorf and Haider (2009) investigated the sequencing aspect of skill learning and found an accuracy strategy to impair the expression of implicit knowledge compared to speed instruction; however, evidence of learning was still detected under accuracy instruction compared to a non-learning control group. Yet, in this experiment, the accumulated sequenceknowledge under speed/accuracy instructions was not compared to a phase where the importance of speed and accuracy was equally emphasized. Such a comparison would reveal whether implicit sequence knowledge is acquired at the same level under different instructions. Recently, Barnhoorn et al. (2019) found that speed instruction benefits the development of representations about repeating sequences while forcing participants to be more accurate leads to a faster selection of responses via better stimulus-response associations. In this study, the participants were aware of the repeating sequences; thus, the learning was completely explicit. The studies mentioned above suggest that speed instruction might benefit sequence learning more than accuracy instruction. These studies used relatively simple, deterministic sequences (i.e., sequences with a simple repeating pattern). Therefore, data are still lacking on whether instruction affects probabilistic representations.

Human participants can rapidly extract statistical information from the environment (Frost et al. 2015). But how fragile are these representations? Previous studies have shown that accelerated learning can be advantageous for habit formation (Hardwick et al. 2019) and also affects the sequencing aspect of skill learning (Hoyndorf and Haider 2009; Barnhoorn et al. 2019). However, these studies could not distinguish whether the instructions affect the representations or momentary performance. Instructing participants to be fast or accurate during the learning process, and test their knowledge after the instructed phase would allow us to decipher whether the statistical representations are themselves fragile or only the performance is affected. If instructions do not affect statistical learning, it will underscore the robust nature of picking up non-adjacent statistical regularities (Kóbor et al. 2017).

Here, we aimed to test whether speed or accuracy instructions affect the acquisition of complex statistical regularities using an implicit probabilistic sequence learning task. We go beyond previous investigations by at least 2 aspects: First, by studying complex probabilistic sequences with non-adjacent secondorder dependencies (Remillard 2008). This feature means that to predict the nth element of the sequence, we need to know the $n-2$ th element instead of $n-1$ th. This structure creates an abstract sequence representation, and its acquisition will be based on statistical regularities (Nemeth et al. 2013), which are also fundamental in complex cognitive skills such as human language (Christiansen and Chater 2015).

The second novel contribution of our study is that we also test the implicit sequence knowledge of our participants after the (instructed) training phase. Our learning task was completed in 2 different phases. In the first phase, we instructed the participants to focus either on accuracy or speed while performing the task (different instruction phase, accuracy vs. speed group). After the training phase, we tested both groups of participants with the same instruction (i.e., focusing both on accuracy and speed, same instruction phase). By doing so, we aimed to differentiate between the effects of instructions on training performance and acquired knowledge. Our questions were 1) whether the speed/accuracy instruction affects the learning of probabilistic statistical regularities, and if yes, 2) do they affect the training performance (different instruction phase) and the retrieval of knowledge (same instruction phase) equally?

\section{Materials and Methods}

\section{Participants}

Sixty-six healthy young adults took part in the study. Five of them were excluded from the experiment because they conceivably misunderstood the instructions. Their performance was more than 2 standard deviations above or below the mean of their group in more than $50 \%$ of the epochs (units of analysis), which was not observable during the practice session. Therefore, 61 participants remained in the final sample (40 females), which is sufficient to detect group differences in statistical learning (see power analysis in the "Justification for sample size" section of the Supplementary Materials). Another 4 participants were excluded from the analysis of the inclusion/exclusion task for not following instructions (see inclusion/exclusion part of the Results section).

Participants were between 19 and 27 years of age $\left(M_{\text {age }}=\right.$ 21.18 years, $\mathrm{SD}_{\text {age }}=2.13$ years $)$. All of them were students from Budapest, Hungary $\left(M_{\text {years of education }}=14.14\right.$ years, $\mathrm{SD}_{\text {years of education }}=$ 1.64 years). Participants had a normal or corrected-to-normal vision, none of them reported a history of any neurological and/or psychiatric disorders, and none of them was taking any psychoactive medication at the time of the experiment. Handedness was measured using the Edinburgh Handedness Inventory (Oldfield 1971). The laterality quotient (LQ) of the sample varied between -84.62 and 100 (-100 indicates complete left-handedness, 100 indicates complete right-handedness, $\mathrm{M}_{\mathrm{LQ}}=62.25, \mathrm{SD}_{\mathrm{LQ}}=53.73$ ). They performed in the normal range on the counting span task $\left(M_{\text {Counting Span }}=3.66, \mathrm{SD}_{\text {Counting Span }}=0.81\right)$ All participants gave written informed consent before enrollment and received course credit for participating. They were randomly assigned to the accuracy group $(n=31)$ or speed group $(n=30)$.

No group differences were observed in terms of age, years of education, handedness, and neuropsychological performance (see Table 1). Males and females were equally represented in the sample (accuracy group: 11 males, speed group: 10 males, $\chi^{2}(1$, $N=61)=0.03, P=0.86)$. The study was approved by the Research Ethics Committee of the Eötvös Loránd University, Budapest, Hungary, and it was conducted in accordance with the Declaration of Helsinki. 
Table 1. Comparison of the 2 groups on age, years of education, handedness, and neuropsychological performance

\begin{tabular}{lccc}
\hline & Accuracy group M(SD) & Speed group M(SD) & t-test \\
\hline Age (years) & $21.29(2.28)$ & $21.07(2.00)$ & $t(59)=-0.41, P=0.69, \mathrm{BF}_{01}=4.82$ \\
Education (years) & $14.31(1.60)$ & $13.97(1.71)$ & $t(59)=-0.80, P=0.43, \mathrm{BF}_{01}=3.87$ \\
Handedness (LQ) & $54.88(55.00)$ & $69.86(52.20)$ & $t(59)=1.09, P=0.28, \mathrm{BF}_{01}=3.02$ \\
Counting span score & $3.69(0.75)$ & $3.64(0.88)$ & $t(59)=0.21, P=0.83, \mathrm{BF}_{01}=5.08$ \\
\hline
\end{tabular}

\section{Alternating Serial Reaction Time Task}

In this study, we used the implicit version of the alternating serial reaction time (ASRT) task (Howard and Howard 1997; Nemeth, Janacsek, Londe, et al. 2010). In the ASRT task, 4 empty circles were presented horizontally in front of a white background in the middle of a computer screen. A target stimulus (drawing of a dog's head) was presented sequentially in one of the 4 empty circles (Fig. 1A). The stimuli were 300 pixels each. The monitor resolution was $1280 \times 1024$ pixels, and the viewing distance from the monitor was approximately $60 \mathrm{~cm}$. A keyboard with 4 heightened keys (Z, C, B, and M on a QWERTY keyboard) was used as a response device, each of the 4 keys corresponding to the circles in a horizontal arrangement. Participants were asked to respond with their middle and index fingers of both hands by pressing the button corresponding to the target position. At the beginning of each block of the ASRT task, the 4 empty circles appeared horizontally on the screen for $200 \mathrm{~ms}$, and then, the first target stimulus occurred, and it remained on the screen until the first correct response. The next stimulus appeared after a $120 \mathrm{~ms}$ response-to-stimulus interval.

The serial order of the 4 possible positions (coded as 1, 2, 3, and 4 in a horizontal arrangement, with 1 as the leftmost and 4 as the rightmost position) in which target stimuli could appear was determined by an eight-element probabilistic sequence. In this sequence, every second element appeared in the same order. In contrast, the other elements' positions were randomly chosen out of the 4 possible locations (e.g., 2r4r3r1r, where $r$ indicates a truly random position). Therefore, some combinations of 3 consecutive trials (triplets) occurred with a higher probability than others. For example, 2X4, 4X3, 3X1, and 1X2 (where "X" indicates any possible middle element of the triplet) would often occur because the third element (bold numbers) could be derived from the sequence (or occasionally could be a random element as well). In contrast, $1 \mathrm{X} 3$ or $4 \mathrm{X} 2$ would occur with lower probability because the third element could only be random (Fig. 1B). Therefore, the third element of a high-probability triplet is more predictable from the first event when compared to a low-probability triplet.

There were 64 possible triplets in the task (4 stimuli combined for 3 consecutive trials). Sixteen of them were high-probability triplets, each of them occurring in approximately $4 \%$ of the trials, 5 times more often than the low-probability triplets. Overall, high-probability triplets occur with approximately $62.5 \%$ probability during the task, while low-probability triplets only occur with a probability of $37.5 \%$ (Fig. 1C).

As participants practice the ASRT task, their responses become faster and more accurate to the high-probability triplets compared to the low-probability triplets, revealing statistical learning throughout the task (Howard and Howard 1997; Song et al. 2007; Kóbor et al. 2017; Unoka et al. 2017). Each block of the ASRT task contained 85 stimuli (5 random trials were presented at the beginning of the block, then the eight-element alternating sequence was repeated 10 times). Each participant performed a randomly selected sequence from the 6 possible original sequences: 2r1r3r4r, 2r1r4r3r, 2r3r4r1r, 2r3r1r4r, 2r4r3r1r, and 2r4r1r3r.

\section{Inclusion-Exclusion Task}

We also administered an inclusion-exclusion task (Destrebecqz and Cleeremans 2001; Destrebecqz et al. 2005; Jiménez et al. 2006; Fu et al. 2010), which is based on the "Process Dissociation Procedure," a widely used method to disentangle the explicitimplicit processes in memory tasks (Jacoby 1991). In the first part of the task, we asked participants in what order the stimuli (both pattern and random elements) appeared during the task and to type the sequence using the same 4 response buttons they used during the ASRT task (inclusion instruction). After that, they had to generate new sequences that were different from the learned sequence (exclusion condition). Both parts consisted of 4 runs, and each run finished after 24 button presses, which is equal to 3 rounds of the eight-element alternating sequence (Kóbor et al. 2017; Horvath et al. 2018; Kiss et al. 2019).

We assessed performance by the occurrence of highprobability triplets in the sequence of responses. Thus, in the inclusion condition, successful performance is indicated by producing high-probability triplets above chance level. It can be achieved solely by implicit knowledge (however, explicit knowledge can also boost performance, but it is not necessary for the successful completion of the task).

On the contrary, successful performance in the exclusion condition (i.e., generating a new sequence that is different from the learned one) is indicated by the production of high-probability triplets at or under chance level. This is only possible if the participant has conscious (explicit) knowledge about the learned statistical regularities, and they can inhibit the production of highprobability triplets consciously. The generation of the learned statistical regularities above chance level, even in the exclusion task, indicates that the participant relies on their implicit knowledge, as it cannot be controlled consciously.

To test whether the participants gained consciously accessible triplet knowledge, first, we calculated the percentage of the generated high-probability triplets in the inclusion and exclusion conditions separately. Then, we tested whether the occurrence of high-probability triplets differed from the probability of generating them by chance. The chance level was considered $25 \%$ because, after 2 consecutive button presses, the chance for the third button press to form a high-probability triplet with the 2 preceding button presses is $1 / 4=25 \%$. We also compared the percentages of the high-probability triplets across conditions (inclusion and exclusion task) and groups (accuracy group and speed group) (for more details about the inclusion-exclusion task, see: Kóbor et al. 2017; Horvath et al. 2018; Kiss et al. 2019).

\section{Questionnaire}

We used a questionnaire to scrutinize whether the participants preferred accuracy or speed in general and whether they were 
A)

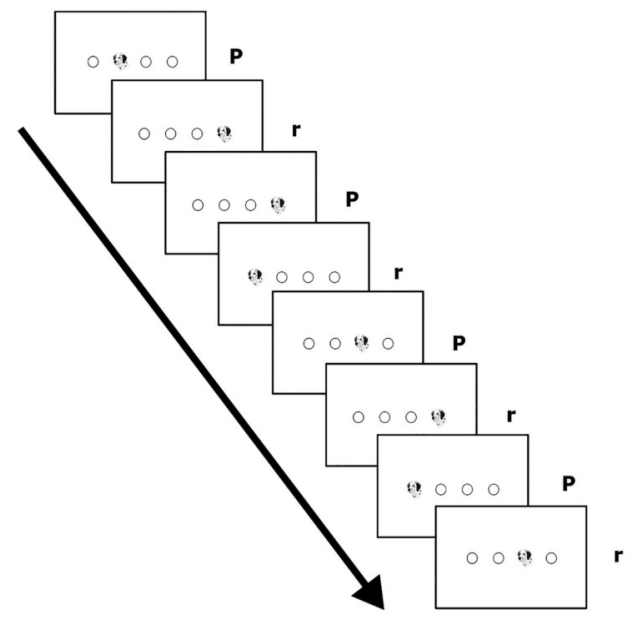

B) SEQUENCE: $2 r 4 r 3 r 1 r$

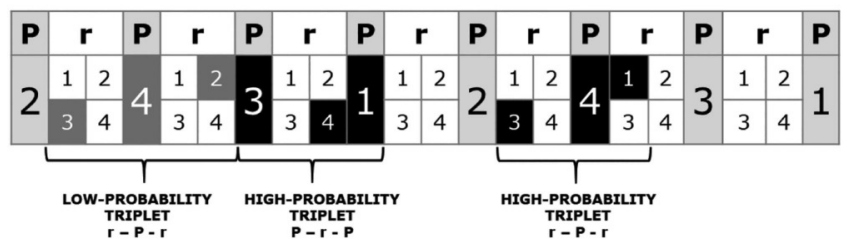

C)

\begin{tabular}{l|c|c|}
\cline { 2 - 3 } & $\begin{array}{c}\text { Structure: } \\
\mathbf{P}-\mathbf{r}-\mathbf{P}\end{array}$ & $\begin{array}{c}\text { Structure: } \\
\mathbf{r}-\mathbf{P}-\mathbf{r}\end{array}$ \\
\hline $\begin{array}{l}\text { High-probability triplets } \\
\mathbf{6 2 . 5} \% \text { of all trials }\end{array}$ & $3-4-1(50 \%)$ & $3-4-1(12.5 \%)$ \\
\hline & & $3-4-2(12.5 \%)$ \\
Low-probability triplets & never occurring (always & $3-4-3(12.5 \%)$ \\
37.5\% of all trials & high) & $3-4-4(12.5 \%)$ \\
\hline
\end{tabular}

D)

\begin{tabular}{|l|}
\hline PRACTICE \\
\hline blocks of \\
practice with \\
random \\
stimuli
\end{tabular}

$1 \mathrm{EPOCH}=5$ blocks

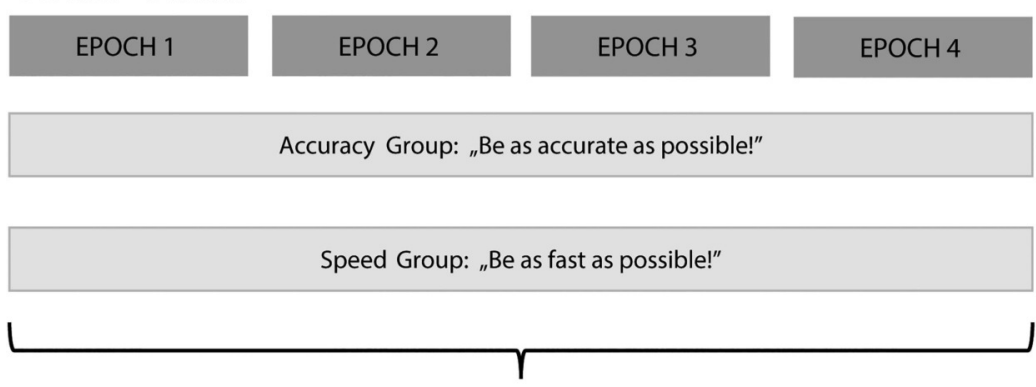

DIFFERENT INSTRUCTION PHASE

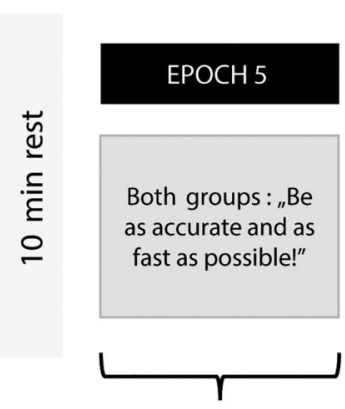

SAME INSTRUCTION PHASE

Figure 1. Task and design of the experiment. (A) Stimulus presentation in the ASRT task. A dog's head appeared in one of the 4 positions. Stimuli appeared in either a pattern $(\mathrm{P})$ or a random $(\mathrm{r})$ position, creating an 8-item long alternating sequence structure. (B) High- and low-probability triplets. Due to the alternating sequence structure, some runs of consecutive stimuli (called triplets) occurred with a higher probability than others. Every trial was defined as the third element of a high- or a low-probability triplet, based on the 2 preceding trials. High-probability triplets can be formed by 2 patterns and 1 random element, but also by 2 random and 1 pattern element. (C) Proportion of high- and low-probability triplets. High-probability triplets occurred in $62.5 \%$ of all trials (of which $50 \%$ came from pattern elements, i.e., from P-r-P structure, and $12.5 \%$ came from random elements, that is, from the r-P-r structure, by chance). Low-probability triplets occurred in the remaining $37.5 \%$ of all trials (of which each individual low-probability triplet occurred with a $12.5 \%$ probability by chance, originating only from the r-P-r structure). (D) Design of the study. In the different instruction phase, different instructions were given to the 2 groups. After 4 epochs (each containing 5 blocks) of the ASRT task, and a 10 min long rest period, the instruction changed. In the fifth epoch (containing 5 blocks of stimuli), the same instruction was given to all of the participants (same instruction phase).

rather accurate or fast in their everyday life. The questionnaire consisted of the following questions: "In an everyday situation, what do you attend more: speed or accuracy (on a scale from 1 to 10 , where 1 means that only the accuracy is important and 10 means that only the speed is important)?", "In an everyday situation, how important is for you to be accurate/fast on a scale from 1 to 10 ?", "According to your friends and family, how fast/accurate are you when you need to solve a problem (on a scale from 1 to 10)?"

\section{Design}

First, the participants completed 3 practice blocks of 85 random trials each to familiarize themselves with the task. After that, the participants completed 2 sessions of the ASRT task. In the training session (referred to as different instruction phase), we gave different instructions to the 2 groups. For the accuracy group, the instruction was to try to be as accurate as possible during the task. On the contrary, the instruction for the speed group was to be as quick as possible. Twenty blocks were presented to the participants in the different instruction phase (for analysis, we organized the blocks into 4 epochs by merging 5 consecutive blocks). Participants could rest a bit after each block. A 10 min rest period was inserted before the second ASRT session. During this period, participants were not involved in any demanding cognitive activity. The second session of ASRT (referred to as the same instruction phase) contained 5 blocks (one epoch). This time, both the accuracy and speed group were instructed to respond to the target stimulus as quickly and as accurately as possible (Fig. 1D). After the ASRT task, the inclusion-exclusion task was administered.

\section{Statistical Analysis}

We defined each trial as the third element of a high- or lowprobability triplet. Trills (e.g., 1-2-1) and repetitions (e.g., 1-1-1) were eliminated from the analysis because participants tended to show preexisting response tendencies to these types of triplets (Howard et al. 2004; Unoka et al. 2017; Janacsek et al. 2018; Takács et al. 2018). The first 5 button presses were random; thus, only the eighth button press could be evaluated as the last element of a valid triplet. Therefore, the first 7 trials were 
excluded from the analysis. Blocks were collapsed into 4 epochs in the different instruction phase (Epoch 1-4), and one epoch in the same instruction phase (Epoch 5) to facilitate data processing and to reduce intraindividual variability. We calculated the median reaction times (RTs) separately for high- and lowprobability triplets for each participant and each epoch. Only correct responses were considered for the RT analysis (we also performed the analyses with the incorrect trials included, see "Analyses including the incorrect trials" in the Supplementary Materials). To ensure that our results on the learning measures were not due to the differences in the average RTs and accuracies, we repeated the analyses with standardized scores (for details, see "Standardized learning scores" section in the Supplementary Materials).

We used mixed-design ANOVAs to compare the learning performance between the 2 groups in the different and same instruction phase. ANOVAs with the within-subject factor of triplet (high- vs. low-probability triplets) and the betweensubjects factor of group (accuracy group vs. speed group) were run (and also with the epoch factor for the analysis of the different instruction phase). In all ANOVAs, the GreenhouseGeisser epsilon $(\varepsilon)$ correction was used if necessary. Corrected $\mathrm{df}$ values and corrected $P$ values are reported (if applicable) along with partial eta-squared $\left(\eta_{p}{ }^{2}\right)$ as the measure of effect size. We used the least significant difference tests for pairwise comparisons. Significant interactions involving the triplet factor were further analyzed using follow-up ANOVAs on the difference scores by the Triplet factor (high-probability triplets vs. lowprobability triplets).

To further support the results of our comparisons, we ran Bayesian t-tests with a standard Cauchy prior distribution $(r=1)$ (Rouder et al. 2009). Here, we report $\mathrm{BF}_{01}$ values: greater values support the null hypothesis over the alternative hypothesis. $\mathrm{BF}_{01}$ values between 1 and 3 indicate anecdotal evidence for $\mathrm{H}_{0}$, while values between 3 and 10 indicate substantial evidence for $\mathrm{H}_{0}$. Values between 1 and 0.33 indicate anecdotal evidence for $\mathrm{H}_{1}$, values between 0.33 and 0.1 indicate substantial evidence for $\mathrm{H}_{1} \cdot \mathrm{BF}_{01}$ values around one do not support either $\mathrm{H}_{0}$ or $\mathrm{H}_{1}$ (Wagenmakers et al. 2011).

To obtain a robust indication of which factors determine performance, we also performed Bayesian repeated-measures ANOVAs on the learning scores (the difference between the 2 levels of triplet factor, i.e., learning scores) (Zavecz et al. 2020). We decided to run the ANOVAs on the learning scores because our primary interest was to quantify the contribution of each interaction to statistical learning rather than to general RTs. Here, we present Bayesian Model Averaging and report the inverted BF inclusion values $\left(1 / \mathrm{BF}_{\text {inclusion }}\right)$. These values indicate the amount of evidence for the exclusion of the given factor from our model. Thus, values below 1 support the inclusion and values above 1 the exclusion of the given factor. Full model comparisons are included in the Supplementary Materials (see "Model comparisons of statistical learning" section in the Supplementary Materials). Cauchy prior distribution was used for the ANOVA with a fixed-effects scale factor of $r=0.5$, and a random-effects scale factor of $r=1$ (JASP Team 2020).

To test whether participants developed conscious knowledge about the learned statistical regularities, we compared the percentage of the generated high-probability triplets in the inclusion-exclusion task to chance level $(25 \%)$ separately for the 2 groups with one-sample t-tests. We compared the percentage of high-probability triplets with a mixed-design ANOVA to reveal whether the level of explicitness differs between groups and conditions.
Additionally, we correlated the average RTs and accuracy scores with the rates of the different items of the questionnaire to check whether the subjective preferences of the participant are related to the ability to follow the instructions.

\section{Results}

\section{Did the Two Groups Perform Equally Before Learning?}

To ensure the lack of substantial preexisting differences between groups in terms of speed or accuracy, we compared the median RTs (only for correct responses) and the accuracy of the 2 groups in the practice session (random stimuli). We did not find differences between groups either in RTs, $t(59)=0.48$, $P=0.64, B_{01}=4.67$, or in accuracy measures, $t(59)=1.08, P=0.28$ $\mathrm{BF}_{01}=3.04$. Therefore, we assumed that there were no preexisting differences between groups regarding their speed or accuracy.

\section{General Speed Changes and Statistical Learning in RT Measures in the Different Instruction Phase}

We investigated how 1) general RTs changed, and 2) whether statistical learning differed between groups during the different instruction phase. RTs were analyzed with a mixed-design ANOVA with the within-subject factors of triplet (high- vs. lowprobability triplets) and epoch (Epoch 1-4), and the betweensubjects factor of group (accuracy group vs. speed group). Please note that main effects and interaction excluding the triplet factor could reveal changes in the average speed/accuracy during the task, independent of the acquisition of statistical regularities, and the main effects and interaction including the triplet factor could unveil differences in statistical learning.

We also compared the learning process with standardized learning scores (see Materials and Methods section). To this end, a mixed-design ANOVA was performed on the standardized RT learning scores with epoch (Epoch 1-4) as a within-subject factor and group (accuracy group vs. speed group) as a betweensubjects factor.

\section{Did the Instruction Affect General RTs in the Different Instruction Phase?}

The main effect of group was significant, $F(1,59)=51.86, P<0.001$, $\eta_{p}{ }^{2}=0.47$, indicating faster overall RTs in the speed group, and the Bayesian comparison of means also favored the difference, $\mathrm{BF}_{01}<0.001$; thus, the instruction did modify the average speed of the participants. A main effect of epoch was found, $F(1.97$, 116.33) $=7.46, P=0.001, \eta_{p}{ }^{2}=0.11$, indicating a change in average RTs during the task: significantly faster RTs were observed between Epoch 2 and Epoch $3(P=0.008)$ as well as between Epoch 3 and Epoch $4(P=0.049)$. The epoch $\times$ group interaction was nonsignificant, $F(1.97,116.33)=2.30, P=0.10, \eta_{p}^{2}=0.04$ (Fig. 2)

\section{Did Statistical Learning Measured by RTs Differ Between Groups in the Different Instruction Phase?}

The main effect of triplet was significant, $F(1,59)=49.41$, $P<0.001, \eta_{p}{ }^{2}=0.46$ : faster RTs were found for high-probability triplets compared to low-probability triplets $\left(\mathrm{BF}_{01}<0.001\right)$, revealing significant implicit statistical learning. Importantly, the triplet $\times$ group interaction was non-significant, $F(1,59)=0.48$, $P=0.49, \eta_{p}{ }^{2}=0.01$ : the degree of learning did not differ between the 2 groups over the course of the learning. The Bayesian 


\section{A) Reaction Time Measures}

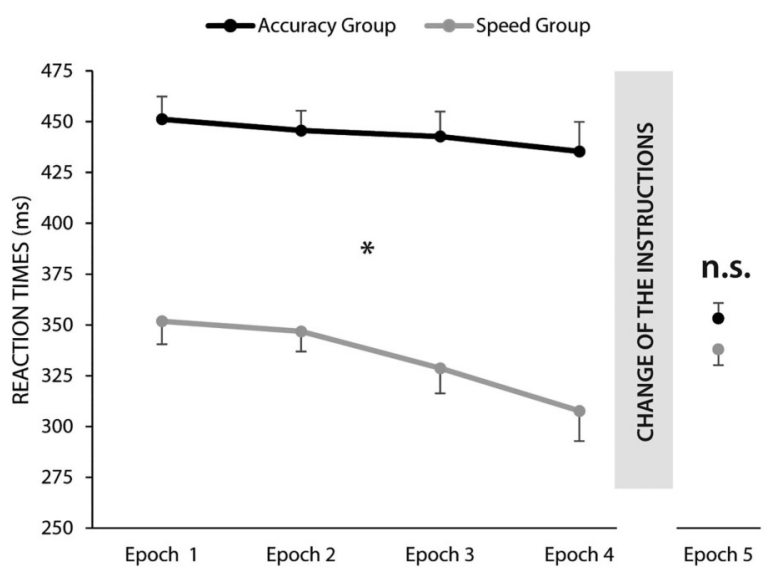

B) Accuracy Measures

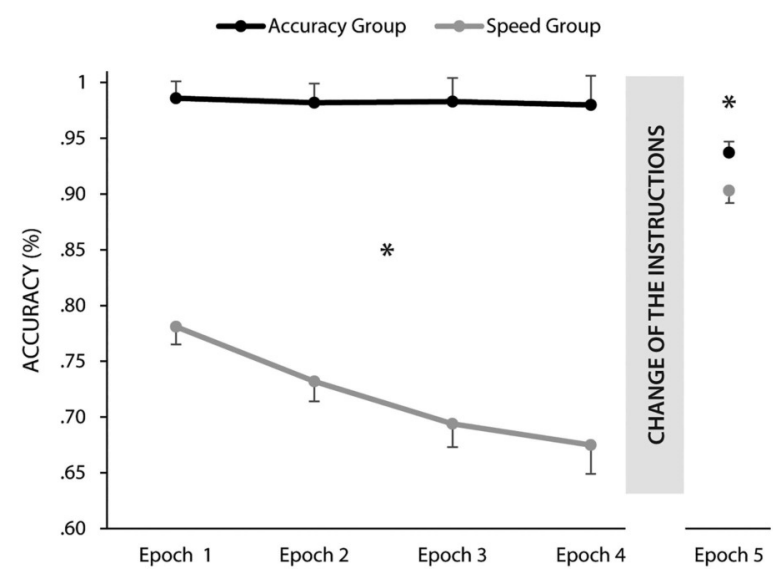

Figure 2. Effects of instruction on (A) average RTs and (B) accuracies. The horizontal axis indicates the 5 epochs of the task and the vertical axis the RTs in milliseconds/accuracies in percentage. The error bars represent the standard error of the mean (SEM). Average RTs were significantly shorter and accuracies lower for the speed group from the first epoch, indicating that the participants followed the instructions. After the change of the instructions (Epoch 5)-although the average scores of the 2 groups approached each other-the difference persisted for accuracies; however, the difference disappeared for the average RTs. $*=P<0.05$, n.s. $=P>0.05$.

comparison of mean differences also supported the lack of difference, $\mathrm{BF}_{01}=4.17$ (Fig. 3). The triplet $\times$ epoch interaction was significant, $F(3,177)=5.66, P=0.001, \eta_{p}^{2}=0.09$ : In the first epoch, independently from groups, no difference was detected between high- and low-probability triplets $(P=0.54)$, and learning (faster RTs for high- than for low-probability triplets) emerged from the second epoch (each $P<0.007$ ). Follow-up analysis on the difference between high- and low-probability triplets (learning scores) revealed an increase in learning scores between Epoch 1 and Epoch $2(P<0.001)$, but not between Epoch 2 and Epoch $3(P=0.90)$ or Epoch 3 and Epoch $4(P=0.17)$. The interaction between the triplet, epoch, and group factors was non-significant, $F(3,177)=0.90, P=0.43, \eta_{p}{ }^{2}=0.02$, revealing no difference in the time course of statistical learning between groups. The analysis with the standardized learning scores in the RT measures revealed similar results (see Supplementary Materials for details).

\section{Bayesian Model Averaging in the Different Instruction Phase in RT Measures}

We conducted a Bayesian repeated-measures ANOVA to quantify the contribution of the different factors to statistical learning (to the difference between the 2 levels of the Triplet factor, that is, low-probability triplets minus high-probability triplets). The ANOVA was performed on the learning scores as the dependent variable, with the within-subject factor of epoch (Epoch 1-4) and the between-subject factor of group (accuracy group vs. speed group). Please note that, because this ANOVA is conducted on learning scores, here the epoch factor corresponds to the triplet $x$ epoch interaction, the group factor to the triplet $x$ group interaction, and the epoch $\times$ group interaction to the three-way interaction of the frequentist ANOVA. The Bayesian ANOVA supported the inclusion of the epoch factor, and the exclusion of the group factor and the epoch $\times$ group interaction (Table 2). This result suggests that although the learning scores changed throughout the task, this change was independent of the instructions, and the overall statistical knowledge was not different between the 2 groups (see detailed model comparisons in Supplementary Table 7).
Table 2. Analysis of effects for the RT learning scores

\begin{tabular}{lccc}
\hline Effects & $P($ incl $)$ & $P($ incl data $)$ & $B_{\text {exclusion }}$ \\
\hline Epoch & 0.60 & 0.98 & 0.04 \\
Group & 0.60 & 0.19 & 6.49 \\
Epoch $\times$ Group & 0.20 & 0.02 & 11.13 \\
\hline
\end{tabular}

Notes: The column "Effects" lists the main effects and interactions. The P(incl) column denotes the prior, and the $\mathrm{P}$ (incl|data) the posterior inclusion probability. The $\mathrm{BF}_{\text {exclusion }}$ column indicates the change from prior to posterior odds.

\section{General Accuracy Changes and Statistical Learning in Accuracy Measures in the Different Instruction Phase}

Next, we repeated the above analyses on accuracy measures to see how 1) general accuracy changed, and 2) whether statistical learning differed between groups during the different instruction phase. We ran a mixed-design ANOVA with the within-subject factors of triplet (high- vs. low-probability triplets) and epoch (Epoch 1-4), and the between-subject factor of group (accuracy group vs. speed group). Please note again that the main effects and interaction excluding the triplet factor could reveal information about the average speed/accuracy during the task, independent of statistical learning, and main effects and interaction including the triplet factor could unveil potential differences in terms of statistical learning.

\section{Did the Instruction Affect General Accuracies in the Different Instruction Phase?}

The main effect of group was significant, $F(1,59)=117.40$, $P<0.001, \eta_{p}{ }^{2}=0.67$, signaling higher average accuracy in the accuracy group; thus, the instructions did influence the accuracy of the participants. The Bayesian comparison of means also supported the difference $\left(\mathrm{BF}_{01}<0.001\right)$. The ANOVA revealed a main effect of epoch, $F\left(1.81,107=8.19, P=0.001, \eta_{p}{ }^{2}=0.13\right.$, revealing a significant decrease in accuracies between Epoch 1 and Epoch $2(P=0.02)$ and between Epoch 2 and Epoch $3(P=0.002)$. The epoch $\times$ group interaction was significant, $F(1.84,107)=7.08$, $P=0.002, \eta_{p}{ }^{2}=0.11$, indicating that accuracy decreased over the 


\section{A) Accuracy Group}

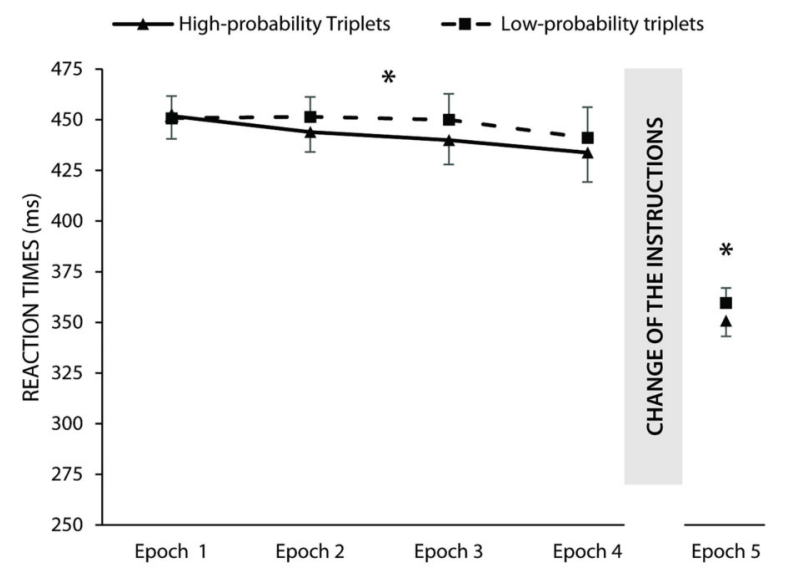

B) Speed Group

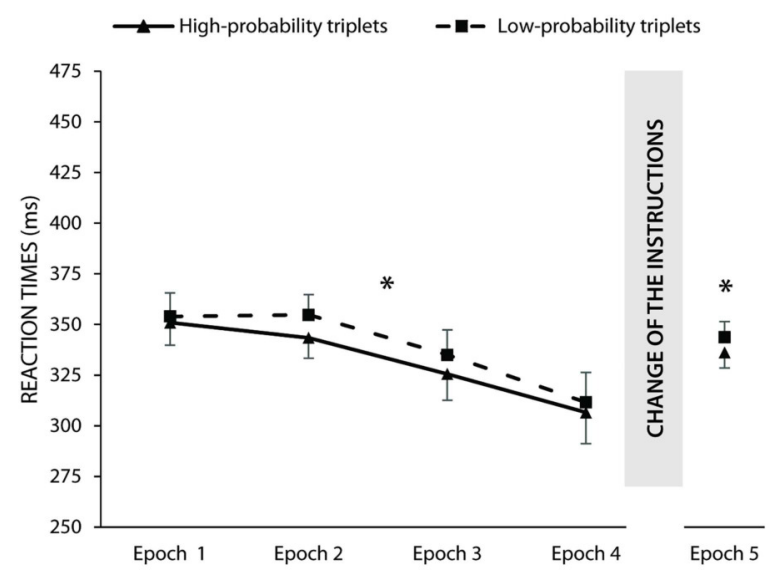

Figure 3. Learning in RT measures in the (A) accuracy group and (B) speed group. The horizontal axis shows the 5 epochs of the task and the vertical axis the RTs. The solid line represents the RTs for the high-probability triplets, while the dashed line indicates the RTs for the low-probability triplets. The error bars represent the SEM. Please note that the gap between the 2 lines indicates the learning of statistical regularities. The RTs for high-probability triplets were smaller for both groups and phases. The difference between the 2 trial types remained after the change of the instructions. A similar level of learning was measured in both groups and phases. $* P<0.05$.

epochs in the speed group (each $P<0.005$, except between Epoch 3 and Epoch $4, P=0.36)$, and it remained similarly high in all epochs in the accuracy group (each $P>0.74$ ) (Fig. 2).

\section{Did Statistical Learning Measured by Accuracies Differ Between Groups in the Different Instruction Phase?}

The main effect of triplet was significant, $F(1,59)=93.88$, $P<0.001, \eta_{p}{ }^{2}=0.61$ : participants responded more accurately to high-probability triplets compared to low-probability triplets, revealing prominent implicit statistical learning also in accuracy measures. The Bayesian comparison also supported the difference, $\mathrm{BF}_{01}<0.001$. Contrary to the $\mathrm{RT}$ results, the ANOVA revealed a significant interaction between the triplet and group factors, $F(1,59)=45.25, P<0.001, \eta_{p}{ }^{2}=0.43$. The speed group responded more accurately to high-probability triplets compared to the low-probability triplets; the accuracy group exhibited similarly accurate responses to the 2 types of triplets $\left(\mathrm{BF}_{01}<0.001\right)$ (Fig. 4). The triplet $\times$ epoch interaction was significant, $F(3,177)=3.69$, $P=0.01, \eta_{p}{ }^{2}=0.06$; thus, the degree of statistical learning changed over the course of learning. Follow-up analysis of the difference between high- and low-probability triplets (learning scores) revealed a decrease in statistical knowledge between Epoch 3 and Epoch $4(P=0.01)$, but not between Epoch 1 and Epoch 2 $(P=0.19)$ or Epoch 2 and Epoch $3(P=0.13)$. The triplet $\times$ epoch $\times$ group interaction was also significant, $F(2.95,174.28)=2.99$, $P=0.03, \eta_{p}{ }^{2}=0.05$, suggesting different dynamics of implicit statistical learning for the 2 groups. The follow-up analysis on the difference between high- and low-probability triplets (learning scores) revealed that in the accuracy group, no change was observed between consecutive epochs (each $P>0.74$ ). On the contrary, in the speed group, an increase was observed between Epoch 2 and Epoch $3(P=0.04)$ and a decrease between Epoch 3 and Epoch $4(P=0.001)$. The analysis with the standardized learning scores in accuracy measures revealed similar results (see Supplementary Materials for details).

\section{Bayesian Model Averaging in the Different Instruction Phase in Accuracy Measures}

We ran a Bayesian repeated-measures ANOVA on the accuracy learning scores with the same factors as for the RT analysis.
Table 3. Analysis of effects for the accuracy learning scores

\begin{tabular}{lccl}
\hline Effects & $P($ incl $)$ & $P($ incl $\mid$ data $)$ & $B F_{\text {exclusion }}$ \\
\hline Epoch & 0.60 & 0.80 & 0.38 \\
Group & 0.60 & 1.00 & $9.50 \mathrm{e}-7$ \\
Epoch $\times$ Group & 0.20 & 0.47 & 0.29
\end{tabular}

Notes: The column "Effects" indicates the main effects and interactions. The $P($ incl) column denotes the prior, and the P(incl|data) the posterior inclusion probability. The $\mathrm{BF}_{\text {exclusion }}$ column indicates the change from prior from posterior odds.

The Bayesian ANOVA indicates that, averaged across all models, the models including the group factor, the epoch factor, and the interaction are more likely. However, the latter 2 improve the model to a much smaller extent compared to the Group factor. This result underscores that the instructions affected statistical learning in accuracy measures, and the dynamic of the learning trajectory is different between the 2 groups (see detailed model comparisons in Supplementary Table 8) (Table 3).

\section{Did the Acquired Knowledge Differ Between Groups in the Same Instruction Phase?}

First, we calculated the median RTs separately for the highand low-probability triplets in the same instruction phase. We analyzed RTs of Epoch 5 with a mixed-design ANOVA with the within-subject factor of triplet (high-probability triplets vs. lowprobability triplets) and the between-subjects factor of group (accuracy group vs. speed group).

A significant main effect of triplet was found, $F(1,59)=50.50$, $P<0.001, \eta_{p}^{2}=0.46$, indicating the emergence of statistical knowledge, as RTs for high-probability triplets were smaller than RTs for low-probability triplets $\left(\mathrm{BF}_{01}<0.001\right)$. The main effect of group did not reach significance, $F(1,59)=2.03, P=0.16, \eta_{p}{ }^{2}=0.03$, indicating that after the change of the instructions, the average RT difference between the 2 groups disappeared; however, the Bayesian comparison revealed only anecdotal evidence for the lack of difference, $\mathrm{BF}_{01}=2.08$ (Fig. 2). Importantly, the triplet $\times$ group interaction did not reach significance, $F(1,59)=0.27$, 


\section{A) Accuracy Group}

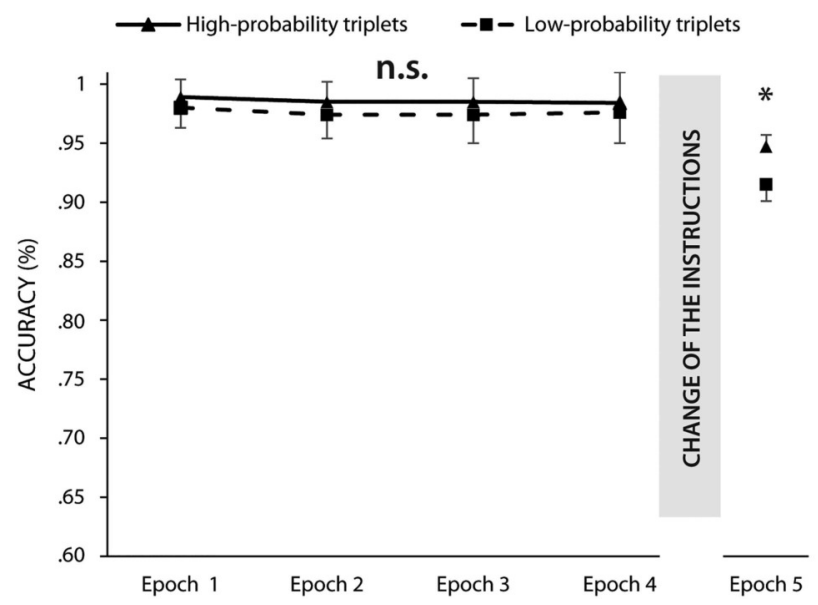

\section{B) Speed Group}

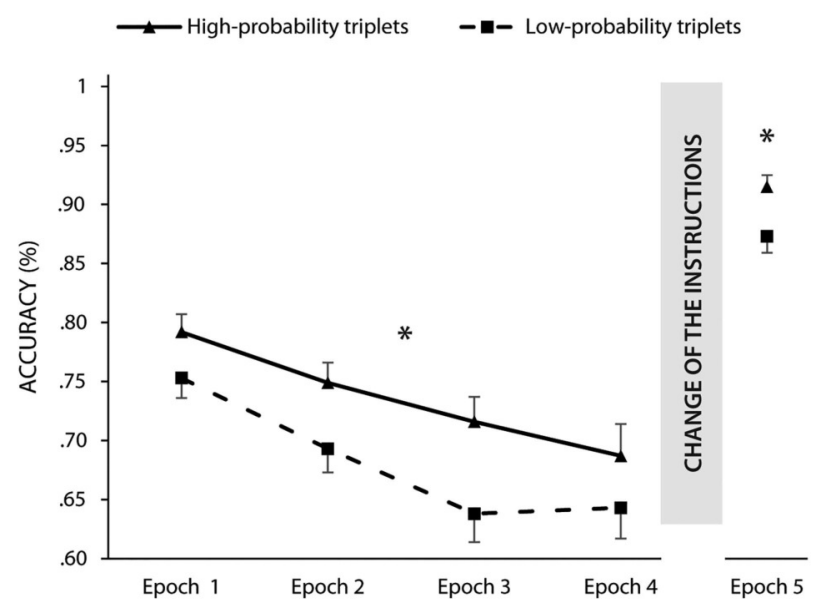

Figure 4. Learning in accuracy measures in the (A) accuracy group and (B) speed group. The horizontal axis shows the 5 epochs of the task and the vertical axis the RTs. The solid line represents the RTs for the high-probability triplets, while the dashed line indicates the RTs for the low-probability triplets. The error bars represent the SEM. Please note that the learning of statistical regularities is measured by the gap between the 2 lines. The accuracies for high-probability triplets were smaller in the speed group, but not in the accuracy group. However, learning was measurable in both groups after the change of the instructions. ${ }^{*}=P<0.05, \mathrm{n} . \mathrm{s} .=\mathrm{P}>0.05$.

$P=0.60, \eta_{p}{ }^{2}=0.01$. It indicates that, irrespective of the instruction during training, the 2 groups showed the same level of statistical knowledge in the same instruction phase (Fig. 5). Moreover, the Bayesian comparison of statistical learning (the difference between high- and low-probability triplets) between groups also favored the lack of difference, $\mathrm{BF}_{01}=4.58$. The analysis with the standardized learning scores in the RT measures revealed similar results (see Supplementary Materials for details).

Next, we repeated the above analysis on the accuracy scores. The triplet $\times$ group ANOVA revealed a significant main effect of triplet, $F(1,59)=39.96, P<0.001, \eta_{p}{ }^{2}=0.40$, indicating statistical knowledge in accuracy as well: more accurate responses for high-probability triplets compared to the low-probability triplets $\left(\mathrm{BF}_{01}<0.001\right)$. The main effect of group was also significant, $F(1$, 59) $=5.08, P=0.03, \eta_{p}{ }^{2}=0.08$, indicating that the overall difference in accuracy persisted after the change of the instructions; however, according to the Bayesian t-test, the difference was only anecdotal $\left(\mathrm{BF}_{01}=0.55\right)$. Importantly, the triplet $\times$ group interaction did not reach significance, $F(1,59)=0.85, P=0.36$, $\eta_{p}{ }^{2}=0.01$, indicating a similar level of statistical knowledge after the change of the instructions (Fig. 5). The Bayesian comparison of statistical learning between groups also supported the lack of difference, $\mathrm{BF}_{01}=3.53$. The analysis with the standardized learning scores in accuracy measures revealed comparable results (see Supplementary Materials for details).

\section{Did the Participants Develop Conscious Knowledge about the Statistical Regularities, and was it Different Between Groups?}

The inclusion-exclusion task was administered to reveal whether the acquired statistical knowledge remained implicit or became explicitly accessible for the participants. We compared the percentage of the generated high-probability triplets to the chance level separately for the 2 groups (see Materials and Methods section for details).

In the accuracy group, 2 participants were excluded from this analysis because they did not follow the instructions. Participants in the accuracy group generated 32.33\% $(0.15 \%$ SEM) high-probability triplets in the Inclusion condition, which is significantly higher than chance level, $t(28)=4.82, P<0.001$, $\mathrm{BF}_{01}=0.002$. In the exclusion condition, they generated $29.81 \%$ ( $0.12 \%$ SEM) high-probability triplets, which is significantly above chance level, $\mathrm{t}(28)=4.04, \mathrm{P}<0.001, \mathrm{BF}_{01}=0.01$, indicating that they could not consciously inhibit the emergence of this knowledge. These results show that in the accuracy group, knowledge about the statistical regularities remained implicit.

In the speed group, 2 participants were excluded because they did not follow the instructions. Participants in the speed group generated $30.34 \%$ (0.15\% SEM) high-probability triplets in the inclusion condition, which is significantly above the chance level, $\mathrm{t}(27)=3.58, P=0.001, \mathrm{BF}_{01}=0.04$. They also generated more highprobability triplets than expected by chance in the exclusion condition, $29.25 \%(0.21 \% \mathrm{SEM}), \mathrm{t}(27)=2.07, \mathrm{P}=0.048, \mathrm{BF}_{01}=0.99$; thus, knowledge about the statistical regularities remained implicit in the speed group.

Furthermore, we compared the differences between groups and tasks with a 2 (condition: inclusion vs. exclusion) $\times 2$ (group: accuracy group vs. speed group) ANOVA. The main effect of condition was not significant, $F(1,55)=1.66, P=0.20, \eta_{p}{ }^{2}=0.03$, indicating that participants did not perform better in either condition, which was confirmed by a Bayesian t-test, $\mathrm{BF}_{01}=4.21$. Thus, the triplet knowledge of the participants remained implicit. The group main effect did not reach significance, $F(1,55)=0.53, P=0.47, \eta_{p}{ }^{2}=0.01$, indicating that the 2 groups performed equally on the 2 tasks, confirmed also by the Bayesian t-test, $\mathrm{BF}_{01}=3.96$. The interaction of the condition and group factors was not significant, $F(1,55)=0.26, P=0.61, \eta_{p}{ }^{2}=0.01$, revealing that the lack of difference between groups was not influenced by the type of task $\left(\mathrm{BF}_{01}=4.47\right)$. To sum up, the 2 groups performed similarly on the task.

\section{Did the Preexisting Preferences of the Participants Affect Their Performance on the Task?}

We used a questionnaire to check whether the subjective preferences on being fast or accurate in real life were related to the ability to follow instructions (see Materials and Methods section for the questions). We correlated the questionnaire scores with the average RTs and accuracy of the participants separately 
Reaction Times Measures

A)

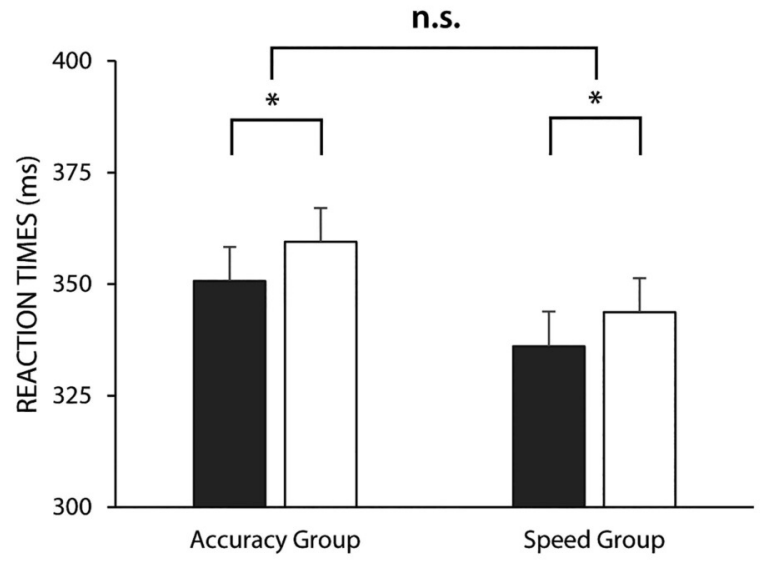

- High-probability triplets $\square$ Low-probability triplets

C)

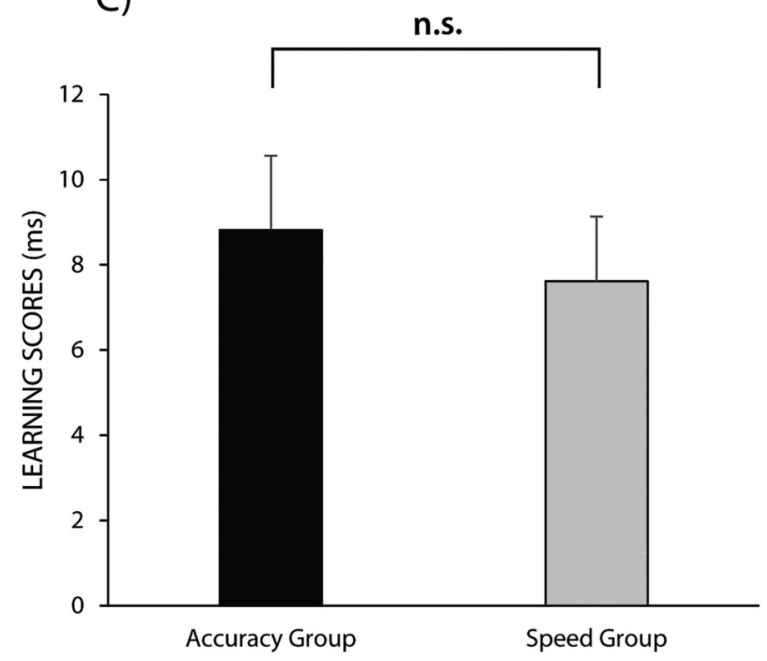

\section{Accuracy Measures}

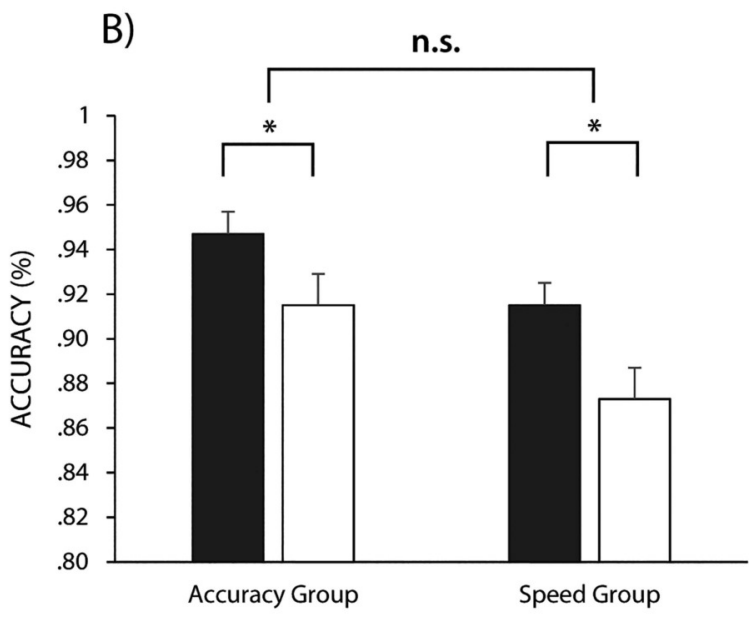

High-probability triplets $\square$ Low-probability triplets

D)

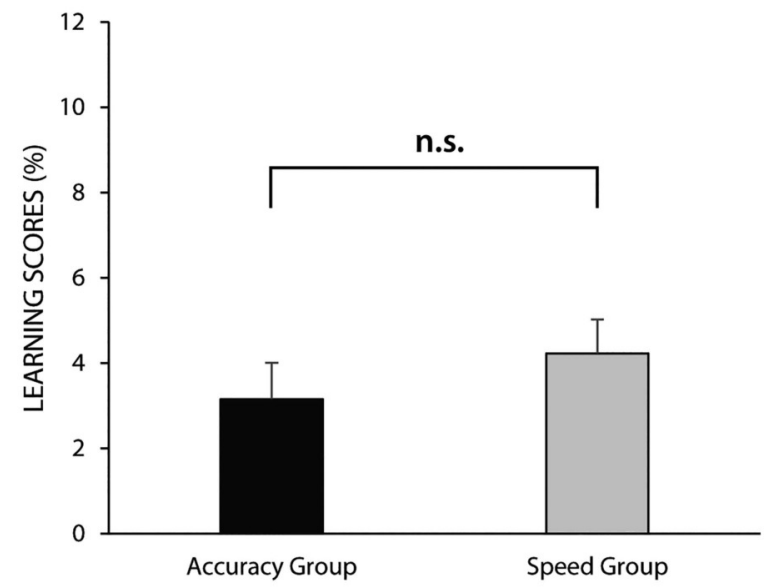

Figure 5. Comparison of the high- and low-probability triplets (A and B), and the learning scores in the same instruction phase. The vertical axis indicates the RTs $(A)$, accuracy $(B)$, or the learning scores (the difference between high- and low-probability triplets, $C$ and $D$ ). The horizontal axis represents the 2 groups. The error bars denote the SEM. Although statistical knowledge was detected in both groups, no significant difference was found in the learning scores, and the lack of difference was confirmed by Bayesian analysis. ${ }^{*}=P<0.05$, n.s. $=P>0.05$.

for the 2 groups. We did not find any significant correlations between the average scores and subjective ratings either in the accuracy group or in the speed group (each $P>0.09$ ). This result indicates that the preference for accuracy or speed, and whether the participants are rather fast or accurate in real life did not play a role in the ability to follow the instructions.

\section{Discussion}

Here, we aimed to unveil whether speed/accuracy instructions can influence an essential component of skill learning, namely the acquisition of probabilistic statistical regularities. To this end, we instructed 2 groups of participants to be either fast or accurate during the training on an implicit probabilistic sequence learning task (different instruction phase). In the testing phase, we assessed the acquired knowledge of probabilistic regularities, and this time, all participants were instructed to be both fast and accurate (same instruction phase). As predicted, the instructions greatly affected the average speed and accuracy of the participants: the speed instructions resulted in faster RTs and a higher number of errors, while the accuracy instructions caused slower average RTs and an almost errorless performance. Despite these differences during training, the statistical learning scores based on RTs were similar between groups. However, statistical learning was not detectable with accuracy instructions. Thus, measured by RTs, the instructions did not affect the acquisition of implicit probabilistic regularities during training. Moreover, no difference between the groups was found in the testing phase. This lack of difference suggests that instructions did not affect either the performance during training or the acquired statistical knowledge. Similar results were obtained when we controlled for the differences in average speed between groups. Moreover, 
Bayesian statistical methods also supported the lack of difference between groups in terms of acquired knowledge.

Our main result is that, irrespective of the strategy used during the training, we detected a similar level of acquired statistical knowledge. This finding has several implications. From a narrower, learning perspective, it suggests that our ability to extract the relevant pieces of statistical information from the environment is so robust that instructions cannot influence it. This conclusion is in accordance with the findings that statistical knowledge persists and remains resistant to interference even after 1 year (Kóbor et al. 2017), is intact in dual-task conditions (Vékony et al. 2019) or in certain disorders characterized by cognitive dysfunctions, such as obstructive sleep apnea (Nemeth et al. 2012; Csabi et al. 2014), sleep-disordered breathing (Csábi et al. 2013, 2016), autism (Nemeth, Janacsek, Balogh, et al. 2010), borderline personality disorder (Unoka et al. 2017) or alcohol dependency (Virag et al. 2015). Deterministic learning tasks test patterns that occur with a $100 \%$ probability over time, while the alteration of the random and pattern elements in the ASRT task creates a noisy, uncertain environment, which is similar to the natural environments of learning in everyday life (Fiser et al. 2010). Our results showed that using complex probabilistic regularities, a similar level of statistical knowledge emerges throughout learning, even when learning occurs under different circumstances and with different strategies.

Another compelling result of our study is that participants in the accuracy instruction condition acquired stable statistical knowledge despite the minimization of motor (response) errors during training. The extent of this statistical knowledge was comparable with the knowledge acquired with the speed instruction. This result is especially interesting in light of the theory claiming that the brain is a Bayesian inference machine (Friston 2010) because our results contradict to the findings that committing errors facilitates learning (Bubic et al. 2010). Our brain learns associations between events through continuous adjustments of the estimated probability distribution, that is, the prior. After a prediction error, the prior should be updated in accordance with new information about the probabilistic structure (Friston 2010). Based on these theories, we would expect a low number of errors to impair the learning process; however, this was not the case in our study. This finding raises the possibility that the motor aspect of prediction errors is not crucial in all circumstances for updating the priors during probabilistic sequence learning. This claim is also supported by other studies reporting statistical and sequence learning without overt errors (Fiser and Aslin 2001; Aslin 2017). However, it is also possible that a similar amount of prediction errors might be detected with other methods, for example, by investigating eye movements (Wills et al. 2007; Le Pelley et al. 2011). The exploration of the role of errors in implicit statistical learning deserves future investigation using eye-tracking and electrophysiological methods.

Measured by RTs, a similar level of statistical learning was found under the speed and accuracy instruction conditions in the training phase. This finding is in contrast with the results of Hoyndorf and Haider (2009), as they reported impaired implicit learning performance with an accuracy strategy. In their study, participants performed a regular and a random task set during a number reduction task. They found that only the participants focusing on speed had increased speed for the regular task set. The authors claimed that the increased monitoring due to the accuracy instruction might have impeded the performance, similarly to the results of skill acquisition studies (Beilock et al. 2004, 2008). However, in the same study, Hoyndorf and Haider (2009) found a preference for the regular task set also in the accuracy group, which they interpreted as the focus on accuracy affects only the expression of implicitly acquired knowledge rather than learning processes per se. This conclusion is in accordance with our results, as we found a similar level of statistical knowledge when we equally emphasized the importance of speed and accuracy after the initial learning. The difference in the training phase might be due to the more complex, probabilistic sequence representations used in our study. They may be more resistant to instructions than deterministic patterns. Similarly, Barnhoorn et al. (2019), who have also found the speed instruction to benefit the development of sequence representations, used simple repeating sequences. Moreover, this study investigated explicit sequence learning processes, while our participants were unaware of their accumulated statistical knowledge. A possible explanation for the difference between the effect of implicit and explicit learning conditions could be that the increased speed covers up the explicitness of the task. As a consequence, the task becomes more implicit, the top-down control reduces, and the learning becomes better. In our study, the learning was entirely implicit; therefore, the speeding up could not improve the level of implicitness. Thus, the learning was similar under speed and accuracy instructions. Future investigations are needed to determine the extent to which the implicit or probabilistic nature of the task affects the lack of speed benefit during training.

Although we found a similar level of the acquired statistical knowledge in accuracy measures, a difference was revealed in the training performance: only the speed instruction resulted in measurable statistical learning. Accuracy is a measure that can reach a maximum of $100 \%$; that is, the task can be performed without errors. Our results suggest that the accuracy instruction caused a ceiling effect. Participants completed the task nearly without error, which did not allow us to measure statistical learning in accuracy measures (i.e., to find a significant difference between responses to high- vs. low-probability triplets). However, learning did occur, evidenced by the results of the testing phase. These findings call for a more careful approach when we evaluate the learning phase in terms of accuracy measures: focusing on being accurate can distort the learning scores of interest so much that, in some instances, we cannot reveal the knowledge that exists.

From a broader cognitive neuroscience perspective, it is essential to highlight the relationship between learning and performance in our study. Most studies in the field of cognitive neuroscience measure learning in a single context, and draw conclusions about brain-behavior relationships based on either "long-term learning" (the relatively permanent changes in knowledge, i.e., competence) or "momentary performance" (the temporary fluctuation in behavior) (e.g., Thomas et al. 2004; Turk-Browne et al. 2010; Rose, Haider, Salari, and Buchel 2011; Heideman, van Ede, and Nobre 2018). However, it was shown that these 2 factors could be separated from each other. For example, learning and performance can differ due to fatigue, different types of practice, latent learning, or overlearning of the practiced skill (Soderstrom and Bjork 2015). Our study also revealed that skill learning competence could differ from the momentary performance due to different instructions, at least when accuracy is used as an indicator. This result draws attention to the problem of using only one session to evaluate learning. For example, if the fatigue or boredom of the participants are different when they concentrate on being fast or accurate, then it can influence the conclusions we draw from our results. However, when the learning score (difference score) is based on RTs, this contingency appears smaller, at least when investigating implicit probabilistic sequence learning. 
Future studies should reveal to what extent this phenomenon is generalizable to other types of learning, such as to more explicit or non-statistical learning tasks. Non-learning tasks should also be tested, as general speed-up and changes in accuracy can be seen over the course of various cognitive tasks requiring fast decision-making. Based on our results, we recommend taking into consideration the possible differences between the measured competence and performance when designing learning studies.

We manipulated the general speed and accuracy of the participants by giving explicit instructions to focus either on speed or accuracy, as previous non-learning cognitive tasks also did (e.g., Osman et al. 2000; Christensen et al. 2001; Ullsperger et al. 2004; Aasen and Brunner, 2016). However, it might be questionable if our results genuinely reflect the effect of instructions on learning. One can argue that the instructions in our study were not strong enough to manipulate the learning strategy and the learning processes because previous studies used more pronounced instructions and feedback to modify the strategy of the participants (Hoyndorf and Haider 2009; Barnhoorn et al. 2019). This possibility seems unlikely because, based on our results, the average speed and accuracy were affected by the instructions. Group differences also emerged in "general skill learning" as 1) participants who focused on their speed showed increasingly faster responses, and 2) participants who focused on their accuracy sustained a high level of accuracy during the learning phase compared to the other group. In contrast to these findings, the acquisition of statistical regularities was not affected by the instructions. To sum up, we found evidence that speed and accuracy affect general skill learning and statistical learning differently.

One could also argue that verbal instructions given at the beginning of the task might not be sufficient to regulate subjects' average speed and accuracy because, as time goes on, participants tend to wane in favor of their response tendencies (Heitz 2014). In other words, they will behave according to their preferences for being accurate or fast on a task. In our case, this change in behavior seems unlikely. First, we found no differences in the average RTs and accuracy scores between groups when the participants practiced the task on random sequences (before we gave distinct instructions to the groups), and second, participants did not become less accurate or slower throughout the task. Therefore, the observed effects should be the result of the instructions. Additionally, we measured the participants' individual preferences on response tendencies using a questionnaire (whether they preferred to be accurate or fast). No correlations were observed between these individual preferences and the average speed and accuracy during the task in either group. These aspects indicate that our results indeed reflect the effect of instructions, and participants did not follow their individually preferred response tendencies during the task.

\section{Conclusion}

Our study investigated the effects of speed and accuracy instructions on an essential component of skill learning, namely, the acquisition of probabilistic regularities. Our main finding is that our ability to pick up statistical regularities in a noisy, uncertain environment is so robust that instructions do not influence it. This result indicates that implicit probabilistic sequence learning is independent of the manipulation of the speed/accuracy tradeoff. Another finding of our study is that learning can occur with an almost $100 \%$ accuracy level as well. This result suggests that statistical learning is at least partly independent of accuracy level, and statistical knowledge about the environmental regularities can be acquired even if no response (motor) errors occur. Our results also raise the possibility that competence and performance can differ in some instances. Accuracy instructions can mask the accumulating statistical knowledge during learning when measured by accuracy, although knowledge does emerge in these cases as well. Future studies investigating whether this robustness is related to the implicit feature of the task or whether different types of learning are affected equally seem warranted.

\section{Supplementary Material}

Supplementary material can be found at Cerebral Cortex Communications online.

\section{Notes}

The authors are grateful to Lucia Nemes, Soma Beres, and Reka Sefcsik for their help in data acquisition. Conflict of Interest: None declared.

\section{Funding}

National Brain Research Program (project 2017-1.2.1-NKP-201700002); Hungarian Scientific Research Fund (NKFIH-OTKA K 128016, PI: D. N., NKFIH-OTKA PD 124148, PI: K.J.); Janos Bolyai Research Fellowship of the Hungarian Academy of Sciences (to K.J. and A.M.); EFOP-3.6.1-16-2016-00008 (to A.M.); IDEXLYON Fellowship of the University of Lyon as part of the Programme Investissements d'Avenir (ANR-16-IDEX-0005) (to D.N).

\section{References}

Aasen IE, Brunner JF. 2016. Modulation of ERP components by task instructions in a cued go/no-go task. Psychophysiology. 53: 171-185.

Aslin RN. 2017. Statistical learning: a powerful mechanism that operates by mere exposure. Wiley Interdiscip Rev Cogn Sci. 8:e1373.

Barnhoorn JS, Panzer S, Godde B, Verwey WB. 2019. Training motor sequences: effects of speed and accuracy instructions. J Mot Behav. 51:540-550.

Beilock SL, Bertenthal BI, Hoerger M, Carr TH. 2008. When does haste make waste? Speed-accuracy tradeoff, skill level, and the tools of the trade. J Exp Psychol Appl. 14:340-352.

Beilock SL, Bertenthal BI, McCoy AM, Carr TH. 2004. Haste does not always make waste: expertise, direction of attention, and speed versus accuracy in performing sensorimotor skills. Psychon Bull Rev. 11:373-379.

Bubic A, Von Cramon DY, Schubotz RI. 2010. Prediction, cognition and the brain. Front Hum Neurosci. 4:1-15.

Christensen CA, Ivkovich D, Drake KJ. 2001. Late positive ERP peaks observed in stimulus-response compatibility tasks tested under speed-accuracy instructions. Psychophysiology. 38:404-416.

Christiansen MH, Chater N. 2015. The language faculty that wasn't: a usage-based account of natural language recursion. Front Psychol. 6:1182.

Conway CM. 2020. How does the brain learn environmental structure? Ten core principles for understanding the neurocognitive mechanisms of statistical learning. Neurosci Biobehav Rev. 112:279-299. 
Csábi E, Benedek P, Janacsek K, Katona G, Nemeth D. 2013. Sleep disorder in childhood impairs declarative but not nondeclarative forms of learning. J Clin Exp Neuropsychol. 35:677-685.

Csábi E, Benedek P, Janacsek K, Zavecz Z, Katona G, Nemeth D. 2016. Declarative and non-declarative memory consolidation in children with sleep disorder. Front Hum Neurosci. 9:709.

Csabi E, Varszegi-Schulz M, Janacsek K, Malecek N, Nemeth D. 2014. The consolidation of implicit sequence memory in obstructive sleep apnea. PLoS One. 9:1-6.

Destrebecqz A, Cleeremans A. 2001. Can sequence learning be implicit? New evidence with the process dissociation procedure. Psychon Bull Rev. 8:343-350.

Destrebecqz A, Peigneux P, Laureys S, Degueldre C, Del Fiore G, Aerts J, Luxen A, Van Der Linden M, Cleermans A, Maquet P. 2005. Neural correlates of implicit and explicit sequence learning: interacting networks revealed. Learn Mem. 12:480-490.

Fiser J, Aslin RN. 2001. Unsupervised statistical learning of higher-order spatial structures from visual scenes. Psychol Sci. 12:499-504.

Fiser J, Berkes P, Orbán G, Lengyel M. 2010. Statistically optimal perception and learning: from behavior to neural representations. Trends Cogn Sci. 14:119-130.

Friston K. 2010. The free-energy principle: a unified brain theory? Nat Rev Neurosci. 11:127-138.

Frost R, Armstrong BC, Siegelman N, Christiansen MH. 2015. Domain generality versus modality specificity: the paradox of statistical learning. Trends Cogn Sci. 19:117-125.

Fu Q, Dienes Z, Fu X. 2010. Can unconscious knowledge allow control in sequence learning? Conscious Cogn. 19:462-474.

Hardwick RM, Forrence AD, Krakauer JW, Haith AM. 2019. Timedependent competition between goal-directed and habitual response preparation. Nat Hum Behav. 3:1252-1262.

Heideman SG, van Ede F, Nobre AC. 2018. Temporal alignment of anticipatory motor cortical beta lateralisation in hidden visualmotor sequences. Eur J Neurosci. 48:2684-2695.

Heitz RP. 2014. The speed-accuracy tradeoff: history, physiology, methodology, and behavior. Front Neurosci. 8:1-19.

Horváth K, Török C, Pesthy O, Nemeth D, Janacsek K. 2019. Intention to learn differentially affects subprocesses of procedural learning and consolidation: Evidence from a probabilistic sequence learning task. bioRxiv. 433243.

Howard JH, Howard DV. 1997. Age differences in implicit learning of higher order dependencies in serial patterns. Psychol Aging. 12:634-656.

Howard DV, Howard JH, Japikse K, DiYanni C, Thompson A, Somberg R. 2004. Implicit sequence learning: effects of level of structure, adult age, and extended practice. Psychol Aging. 19:79-92.

Hoyndorf A, Haider H. 2009. The "not letting go" phenomenon: accuracy instructions can impair behavioral and metacognitive effects of implicit learning processes. Psychol Res. 73: 695-706.

Jacoby LL. 1991. A process dissociation framework: separating automatic from intentional uses of memory. J Mem Lang. 30: 513-541.

Janacsek K, Borbély-Ipkovich E, Nemeth D, Gonda X. 2018. How can the depressed mind extract and remember predictive relationships of the environment? Evidence from implicit probabilistic sequence learning. Prog Neuropsychopharmacol Biol Psychiatry. 81:17-24.

Janacsek K, Fiser J, Nemeth D. 2012. The best time to acquire new skills: age-related differences in implicit sequence learning across the human lifespan. Dev Sci. 15:496-505.

JASP Team. 2020. JASP (Version 0.13.1)[Computer software].
Jiménez L, Vaquero JMM, Lupiáñez J. 2006. Qualitative differences between implicit and explicit sequence learning. J Exp Psychol Learn Mem Cogn. 32:475-490.

Kiss M, Nemeth D, Janacsek K. 2019. Stimulus presentation rates affect performance but not the acquired knowledge-evidence from procedural learning. bioRxiv. 650598.

Kóbor A, Janacsek K, Takács A, Nemeth D, Kobor A, Janacsek K, Takacs A, Nemeth D. 2017. Statistical learning leads to persistent memory: evidence for one-year consolidation. Sci Rep. 7:1-10.

Le Pelley ME, Beesley T, Griffiths O. 2011. Overt attention and predictiveness in human contingency learning. J Exp Psychol Anim Behau Process. 37:220-229.

Nemeth D, Csábi E, Janacsek K, Várszegi M, Mari Z. 2012. Intact implicit probabilistic sequence learning in obstructive sleep apnea. J Sleep Res. 21:396-401.

Nemeth D, Janacsek K, Balogh V, Londe Z, Mingesz R, Fazekas M, Jambori S, Danyi I, Vetro A. 2010a. Learning in autism: implicitly superb. PLoS One. 5:1-7.

Nemeth D, Janacsek K, Király K, Londe Z, Németh K, Fazekas K, Adam I, Király E, Csányi A. 2013. Probabilistic sequence learning in mild cognitive impairment. Front Hum Neurosci. 7:318.

Nemeth D, Janacsek K, Londe Z, Ullman MT, Howard DV, Howard JH. 2010b. Sleep has no critical role in implicit motor sequence learning in young and old adults. Exp Brain Res. 201: 351-358.

Oldfield RC. 1971. The assessment and analysis of handedness: the Edinburgh inventory. Neuropsychologia. 9:97-113.

Osman A, Lou L, Muller-Gethmann H, Rinkenauer G, Mattes S, Ulrich R. 2000. Mechanisms of speed-accuracy tradeoff: evidence from covert motor processes. Biol Psychol. 51: 173-199.

Remillard G. 2008. Implicit learning of second-, third-, and fourthorder adjacent and nonadjacent sequential dependencies. QJ Exp Psychol. 61:400-424.

Rose M, Haider H, Salari N, Buchel C. 2011. Functional dissociation of hippocampal mechanism during implicit learning based on the domain of associations. J Neurosci. 31: 13739-13745.

Rouder JN, Speckman PL, Sun D, Morey RD, Iverson G. 2009. Bayesian t tests for accepting and rejecting the null hypothesis. Psychon Bull Rev. 16:225-237.

Soderstrom NC, Bjork RA. 2015. Learning versus performance: an integrative review. Perspect Psychol Sci. 10:176-199.

Song S, Howard JH, Howard DV. 2007. Sleep does not benefit probabilistic motor sequence learning. J Neurosci. 27:1247512483.

Takács Á, Kóbor A, Chezan J, Éltető N, Tárnok Z, Nemeth D, Ullman MT, Janacsek K. 2018. Is procedural memory enhanced in Tourette syndrome? Evidence from a sequence learning task. Cortex. 100:84-94.

Thomas KM, Hunt RH, Vizueta N, Sommer T, Durston S, Yang Y, Worden MS. 2004. Evidence of developmental differences in implicit sequence learning: an fMRI study of children and adults. J Cogn Neurosci. 16:1339-1351.

Turk-Browne NB, Scholl BJ, Johnson MK, Chun MM. 2010. Implicit perceptual anticipation triggered by statistical learning. J Neurosci. 30:11177-11187.

Ullsperger M, Bylsma LM, Botvinick MM. 2004. The conflictadaptation effect : it's not just priming. Cogn Affect Behav Neurosci. 5:467-472.

Unoka Z, Vizin G, Bjelik A, Radics D, Nemeth D, Janacsek K. 2017. Intact implicit statistical learning in borderline personality disorder. Psychiatry Res. 255:373-381. 
Vékony T, Török L, Pedraza F, Schipper K, Plèche C, Tóth L, Janacsek K, Nemeth D. 2020. Retrieval of a well-established skill is resistant to distraction: evidence from an implicit probabilistic sequence learning task. bioRxiv. 849729.

Virag M, Janacsek K, Horvath A, Bujdoso Z, Fabo D, Nemeth D. 2015. Competition between frontal lobe functions and implicit sequence learning: evidence from the long-term effects of alcohol. Exp Brain Res. 233:2081-2089.

Wagenmakers E, Wetzels R, Borsboom D, van der Maas HLJ. 2011. Why psychologists must change the way they analyze their data: the case of psi: comment on Bem (2011).J Pers Soc Psychol. 100:426-432.

Wills AJ, Lavric A, Croft GS, Hodgson TL. 2007. Predictive learning, prediction errors, and attention: evidence from eventrelated potentials and eye tracking. J Cogn Neurosci. 19: 843-854.

Zavecz Z, Horváth K, Solymosi P, Janacsek K, Nemeth D. 2020. Frontal-midline theta frequency and probabilistic learning: a transcranial alternating current stimulation study. Behav Brain Res. 393:112733. 


\section{Supplementary Materials}

\section{Speed or accuracy instructions during skill learning do not affect the acquired \\ knowledge}

Teodóra Vékony ${ }^{\mathrm{a}}$, Hanna Marossy ${ }^{\mathrm{b}}$, Anita Must $^{\mathrm{c}}$, László Vécsei ${ }^{\mathrm{a}, \mathrm{d}}$, Karolina Janacsek $^{\mathrm{b}, \mathrm{e}, \mathrm{f}^{*}}$ \& Dezső Németh $^{\text {b,e, } \text { g* }^{*}}$

${ }^{a}$ Department of Neurology, University of Szeged, Szeged, Semmelweis utca 6, 6725 Szeged, Hungary

b Institute of Psychology, ELTE Eötvös Loránd University, Izabella utca 46, 1064, Budapest, Hungary

${ }^{c}$ Institute of Psychology, University of Szeged, Egyetem utca 2, 6722 Szeged, Hungary

${ }^{\mathrm{d}}$ MTA-SZTE Neuroscience Research Group, University of Szeged, Semmelweis u. 6, 6725 Szeged, Hungary

e Brain, Memory and Language Research Group, Institute of Cognitive Neuroscience and Psychology, Research Centre for Natural Sciences, Magyar tudósok körútja 2, 1117 Budapest, Hungary

${ }^{\mathrm{f}}$ Centre for Thinking and Learning, Institute for Lifecourse Development, School of Human Sciences, Faculty of Education, Health and Human Sciences, University of Greenwich, Old Royal Naval College, 150 Dreadnought, Park Row, SE10 9LS, London, United Kingdom

${ }^{g}$ Lyon Neuroscience Research Center (CRNL), INSERM, CNRS, Université Claude Bernard Lyon 1, Centre Hospitalier Le Vinatier - Bâtiment 462 - Neurocampus 95 boulevard Pinel 69675 Bron, France

* These authors contributed equally to this work.

\section{Author Note}

Correspondence concerning this article should be addressed to Dezso Nemeth, Lyon Neuroscience Research Center (CRNL), INSERM, CNRS, Université de Lyon, Centre Hospitalier Le Vinatier - 
Bâtiment 462 - Neurocampus 95 boulevard Pinel 69675 Bron, France. E-mail: dezso.nemeth@univ-lyon1.fr Phone: +33 481106546 


\section{Justification for sample size}

We ran a power analysis with $G^{*}$ Power 3.1.9.7 to justify our sample size (Faul et al. 2007). We calculated the required sample size based on the results of previously published ASRT studies with a significant interaction between the within-subject factor of Triplet (high- vs. lowprobability) and the between-subjects factor of Group with two independent groups (Nemeth, Janacsek, and Fiser 2013; Nemeth, Janacsek, Király, et al. 2013; Nemeth, Janacsek, Polner, et al. 2013; Virag et al. 2015). The effect sizes ranged from a $\eta_{\mathrm{p}}{ }^{2}$ effect size of .12 to .35 ; therefore, we estimated a $\eta_{\mathrm{p}}{ }^{2}$ effect size of .21 (mean of the previous effect sizes). With an alpha level of .05 and the desired power level of .90 , a total sample size of at least 46 participants is required to detect significant group differences in triplet learning, and 54 participants are required with a power level of .95. Moreover, if we calculate with the lowest expected effect size $\left(\eta_{\mathrm{p}}{ }^{2}=.12\right)$, we would need 60 participants to find a difference with a power of .80 , which is the commonly recommended value (Ellis 2010). Therefore, our sample of 61 participants should be sufficient to detect group differences in triplet learning. 


\section{Model comparisons of statistical learning}

Supplementary Table 1. Bayesian model comparisons for RT learning scores

\begin{tabular}{lccccc}
\hline \multicolumn{1}{c}{ Models } & $\mathbf{P}(\mathbf{M})$ & $\mathbf{P}(\mathbf{M} \mid \mathbf{d a t a})$ & $\mathbf{B F}_{\mathbf{M}}$ & $\mathbf{B F}_{\mathbf{0 1}}$ & error \% \\
\hline Null model (incl. subject) & 0.20 & 0.02 & 0.08 & 1.00 & \\
Epoch & 0.20 & 0.79 & 15.38 & 0.02 & 0.56 \\
Epoch + Group & 0.20 & 0.16 & 0.77 & 0.12 & 1.17 \\
Epoch + Group + Epoch $\times$ Group & 0.20 & 0.02 & 0.09 & 0.85 & 1.70 \\
Group & 0.200 & 0.004 & 0.02 & 4.86 & 1.21 \\
\end{tabular}

Note. All models include subject. The Models column indicates the predictors included in each model, the P(M) column the prior model probability, the $\mathrm{P}\left(\mathrm{M} \mid\right.$ data) column the posterior model probability, the $\mathrm{BF}_{\mathrm{M}}$ column the posterior model odds, and the $\mathrm{BF}_{01}$ column the Bayes factors of all models compared to the null model. The error is an estimate of the numerical error in the computation of the Bayes factor.

Supplementary Table 2. Bayesian model comparisons for accuracy learning scores

\begin{tabular}{lrrrrr}
\hline \multicolumn{1}{c}{ Models } & $\mathbf{P}(\mathbf{M})$ & $\mathbf{P}(\mathbf{M} \mid \mathbf{d a t a})$ & $\mathbf{B F}$ M & $\mathbf{B F}_{\mathbf{0 1}}$ & error \% \\
\hline Null model (incl. subject) & 0.20 & $2.52 \mathrm{e}-7$ & $1.01 \mathrm{e}-6$ & 1.00 & \\
Epoch + Group + Epoch $\times$ Group & 0.20 & 0.47 & 3.50 & $5.40 \mathrm{e}-7$ & 2.42 \\
Epoch + Group & 0.20 & 0.33 & 1.98 & $7.61 \mathrm{e}-7$ & 2.73 \\
Group & 0.20 & 0.20 & 1.01 & $1.25 \mathrm{e}-6$ & 2.24 \\
Epoch & 0.20 & $3.81 \mathrm{e}-7$ & $1.52 \mathrm{e}-6$ & 0.66 & 0.47
\end{tabular}

Note. All models include subject. The Models column indicates the predictors included in each model, the P(M) column the prior model probability, the $\mathrm{P}\left(\mathrm{M} \mid\right.$ data) column the posterior model probability, the $\mathrm{BF}_{\mathrm{M}}$ column the posterior model odds, and the $\mathrm{BF}_{01}$ column the Bayes factors of all models compared to the null model. The error is an estimate of the numerical error in the computation of the Bayes factor. 


\section{Standardized learning scores}

The instructions in the experiment could cause significant differences in the average RTs and accuracies (i.e., all valid trials collapsed together) between the two experimental groups. To ensure that our results on the learning measures were not due to the differences in the average RTs and accuracies, we repeated all our primary analyses with standardized scores. To this end, we divided the learning scores (median RTs for low-probability triplets minus median RTs for highprobability triplets) by the average of RTs for the high- and low-probability triplets of the given epoch, for each participant and each epoch. Similarly, we divided the learning scores (mean accuracy for high-probability triplets minus mean accuracy for low-probability triplets) by the average of accuracies for the high- and low-probability triplets of the given epoch, for each participant and each epoch. RT and accuracy standardized learning scores were analyzed with mixed-design ANOVAs with the within-subject factor of Epoch (Epoch 1 to 4), and the betweensubjects factor of Group (Accuracy Group vs. Speed Group).

\section{Results of the Different Instruction Phase}

\section{RT measures}

First, we compared the standardized learning scores between the two groups in the Different Instruction Phase. The main effect of Group was not significant, $F(1,59)=0.40, p=.53$, $\eta_{\mathrm{p}}{ }^{2}=.01$, indicating that, in accordance with the results of the non-standardized data, the two groups exhibited similar learning scores in the task. The Bayesian comparison of means also supported the lack of difference, $\mathrm{BF}_{01}=4.32$. The ANOVA revealed a main effect of Epoch, $F(2.24,132.58)=2.99, p=.048, \eta_{\mathrm{p}}^{2}=.05$, suggesting that, in accordance with the nonstandardized data, learning scores changed during the task: they became larger from Epoch 1 and 
Epoch $2(p=.005)$, but remained unchanged after that (each $p>.09)$. The interaction of Epoch and Group did not reach significance, $F(3,177)=1.39, p=.25, \eta_{\mathrm{p}}{ }^{2}=.02$, indicating the lack of significant group differences in the dynamics of learning over the epochs.

\section{Bayesian Model Averaging in the Different Instruction Phase in standardized RT}

measures

We conducted a Bayesian repeated-measures ANOVA on the standardized learning scores with the within-subject factor of Epoch (Epoch 1-4) and the between-subjects factor of Group (Accuracy Group vs. Speed Group). The Bayesian ANOVA revealed anecdotal evidence for the inclusion of the Epoch factor, substantial evidence for the exclusion Group factor, and strong evidence for the exclusion of the interaction (Supplementary Table 3). This result suggests that the change of performance was independent of the instructions, and the overall statistical knowledge was not different between groups. The model comparisons can be found in Supplementary Table 4.

\section{Supplementary Table 3. Analysis of effects of the standardized RT learning scores}

\begin{tabular}{lcccr}
\hline & Effects & P(incl) & P(incl|data) & BF exclusion \\
\hline Epoch & 0.60 & 0.54 & 1.27 \\
Group & 0.60 & 0.18 & 6.80 \\
Epoch $\times$ Group & 0.20 & 0.02 & 11.66
\end{tabular}

Note: The column Effects indicates the main effects and interactions. The P(incl) column denotes the prior, and the $\mathrm{P}$ (incl|data) the posterior inclusion probability. The $\mathrm{BF}_{\text {exclusion }}$ column indicates the change from prior to posterior odds. 


\section{Supplementary Table 4. Bayesian model comparisons for standardized RT learning scores}

\begin{tabular}{lccccc}
\hline \multicolumn{1}{c}{ Models } & $\mathbf{P}(\mathbf{M})$ & $\mathbf{P}(\mathbf{M} \mid \mathbf{d a t a})$ & $\mathbf{B F}$ м & BF 01 & error \% \\
\hline Null model (incl. subject) & 0.20 & 0.38 & 2.49 & 1.00 & \\
Epoch & 0.20 & 0.44 & 3.09 & 0.88 & 0.73 \\
Epoch + Group & 0.20 & 0.08 & 0.37 & 4.55 & 1.82 \\
Group & 0.20 & 0.08 & 0.33 & 5.08 & 2.01 \\
Epoch + Group + Epoch $\times$ Group & 0.20 & 0.02 & 0.09 & 18.25 & 1.27 \\
& & & & & \\
\hline
\end{tabular}

Note. All models include subject. All models include subject. The Models column indicates the predictors included in each model, the $\mathrm{P}(\mathrm{M})$ column the prior model probability, the $\mathrm{P}(\mathrm{M} \mid$ data) column the posterior model probability, the $\mathrm{BF}_{\mathrm{M}}$ column the posterior model odds, and the $\mathrm{BF}_{01}$ column the Bayes factors of all models compared to the null model. The error is an estimate of the numerical error in the computation of the Bayes factor.

\section{Accuracy measures}

We also compared the standardized accuracy learning scores between the two groups in the Different Instruction Phase. Importantly, the main effect of Group was significant, $F(1,59)=$ 46.17, $p<.001, \eta_{\mathrm{p}}{ }^{2}=.44:$ the Speed Group showed learning in accuracy measures, while the Accuracy Group did not $\left(\mathrm{BF}_{01}<0.001\right)$. A significant main effect of Epoch was found, $F(3,177)$ $=5.21, p=.002, \eta_{\mathrm{p}}^{2}=.08$. The pairwise comparisons revealed a significant decrease between Epoch 3 and Epoch $4(p=.01)$. The Epoch $\times$ Group interaction was also significant, $F(3,177)=$ 4.82, $p=.003, \eta_{\mathrm{p}}^{2}=.08$. The pairwise comparisons revealed no change in learning scores in the Accuracy Group (each $p>$.87). On the contrary, in the Speed Group, we found a change in learning scores between Epoch 1 and Epoch $2(p=.02)$, between Epoch 2 and Epoch $3(p=.01)$, and between Epoch 3 and $4(p=.001)$. 
Bayesian Model Averaging in the Different Instruction Phase in standardized accuracy measures

We ran a Bayesian repeated-measures ANOVA on the standardized accuracy learning scores with the same factors as for the RT analysis. Averaged across all models, the Bayesian ANOVA strongly supported the inclusion of the Group factor, but also the inclusion of the Epoch factor and the Epoch $\times$ Group interaction (Supplementary Table 5). The results support that statistical learning in accuracy measures is determined by the instructions, and the learning trajectory is different between groups. The model comparisons can be found in Supplementary Table 6.

Supplementary Table 5. Analysis of effects of the standardized accuracy learning scores

\begin{tabular}{lcccr}
\hline & Effects & P(incl) & P(incl|data) & BF exclusion \\
\hline Epoch & 0.60 & 0.99 & 0.01 \\
Group & 0.60 & 1.00 & $9.87 \mathrm{e}-8$ \\
Epoch $\times$ Group & 0.20 & 0.92 & 0.02
\end{tabular}

Note: The column Effects indicates the main effects and interactions. The P(incl) column denotes the prior, and the $\mathrm{P}\left(\right.$ incl|data) the posterior inclusion probability. The $\mathrm{BF}_{\text {exclusion }}$ column indicates the change from prior from posterior odds.

Supplementary Table 6. Bayesian model comparisons for standardized accuracy learning scores

\begin{tabular}{lcrrrr}
\hline \multicolumn{1}{c}{ Models } & P(M) & P(M|data) & BF $\mathbf{M}$ & BF 01 & error \% \\
\hline Null model (incl. subject) & 0.20 & $7.91 \mathrm{e}-9$ & $3.16 \mathrm{e}-8$ & 1.00 & \\
Epoch + Group + Epoch $\times$ Group & 0.20 & 0.92 & 45.95 & $8.60 \mathrm{e}-9$ & 2.66 \\
Epoch + Group & 0.20 & 0.07 & 0.31 & $1.12 \mathrm{e}-7$ & 0.74 \\
Group & 0.20 & 0.01 & 0.04 & $8.49 \mathrm{e}-7$ & 1.77
\end{tabular}


Supplementary Table 6. Bayesian model comparisons for standardized accuracy learning scores

\begin{tabular}{lcrrrrr}
\hline & Models & P(M) & P(M|data $)$ & BF $\mathbf{M}$ & BF 01 & error \% \\
\hline Epoch & & & & & & \\
\hline
\end{tabular}

Note. All models include subject. All models include subject. The Models column indicates the predictors included in each model, the $\mathrm{P}(\mathrm{M})$ column the prior model probability, the $\mathrm{P}(\mathrm{M} \mid$ data) column the posterior model probability, the $\mathrm{BF}_{\mathrm{M}}$ column the posterior model odds, and the $\mathrm{BF}_{01}$ column the Bayes factors of all models compared to the null model. The error is an estimate of the numerical error in the computation of the Bayes factor.

Results of the Same Instruction Phase

We compared the acquired knowledge of the two groups in RT measures with standardized learning scores, and no difference was found between groups, $t(59)=-0.58, p=.57, \mathrm{BF}_{01}=4.46$. We also compared the acquired knowledge of the two groups in accuracy measures, and we did not find differences between the two groups, $t(59)=0.89, p=.38, \mathrm{BF}_{01}=3.61$. The Bayes factors also support the lack of difference for both comparisons. 


\section{Analyses including the incorrect trials}

We analyzed RTs also with the incorrect trials included. For the analysis of the Different Instruction Phase, we ran a mixed-design ANOVA with the within-subject factors of Triplet (highvs. low-probability triplets) and Epoch (Epoch 1 to 4) and the between-subjects factor of Group (Accuracy Group vs. Speed Group).

\section{Different Instruction Phase}

The main effect of Group was highly significant, $F(1,59)=58.85, p<.001, \eta_{\mathrm{p}}^{2}=.50$, indicating that the Speed Group was faster than the Accuracy Group. The Bayesian comparison of means also favored the difference, $\mathrm{BF}_{01}<0.001$. The main effect of Epoch was significant, $F(3,177)=7.23, p<.001, \eta_{\mathrm{p}}^{2}=.11$, indicating a change in RTs over the course of the task. The pairwise comparisons revealed that there was no difference in RTs between Epoch 1 and Epoch 2 $(p=.41)$; however, after that, RTs became faster between every consecutive epoch (each $p<.01)$. The interaction between the Epoch and Group factors was approaching significance, $F(1.96$, $115.70)=2.41, p=.10, \eta_{\mathrm{p}}^{2}=.04$

The main effect of Triplet was significant, $F(1,59)=24.04, p<.001, \eta_{\mathrm{p}}{ }^{2}=.29$ : faster RTs were found for high-probability triplets compared to low-probability triplets $\left(\mathrm{BF}_{01}=0.001\right)$. The interaction between Triplet and the Group factors was significant, $F(1,59)=7.46, p=.008, \eta_{\mathrm{p}}{ }^{2}=$ $.11, \mathrm{BF}_{01}=0.20$ : only the Accuracy Group showed learning $(p<.001)$, and learning was not measurable in the Speed Group $(p=.13)$ (Supplementary Figure 1). The interaction between the Epoch and Triplet factors was also significant, $F(3,177)=5.14, p=.002, \eta_{\mathrm{p}}^{2}=.08$ : post-hoc comparisons revealed that triplet learning was not detectable in the first epoch $(p=.35)$, but in the remaining epochs $(p<.001$ in Epoch 2 and Epoch 3, and trend-level difference in Epoch 4, $p=$ 
.06). The interaction between Epoch, Triplet, and Group was not significant, $F(80.92,171.90)=$ $1.06, p=.37, \eta_{\mathrm{p}}^{2}=.02$.

We repeated the analyses with standardized scores. The main effect of Group was significant, $F(1,59)=6.47, p=.01, \eta_{\mathrm{p}}^{2}=.10$, because learning was higher for the Accuracy Group. The Bayesian comparison of means also supported the difference between groups, $\mathrm{BF}_{01}=0.31$. The main effect of Epoch was significant, $F(3,177)=3.21, p=.02, \eta_{\mathrm{p}}{ }^{2}=.05$, indicating a difference in statistical learning between epochs. The pairwise comparisons revealed a difference between Epoch 1 and Epoch $2(p=.01)$, but not between other two consecutive epochs (each $p>.33)$. The interaction between Epoch and Group was not significant, $F(3,177)=0.39, p=.76, \eta_{\mathrm{p}}^{2}=.01$, indicating that statistical learning was not changing differently between the two groups over the course of the session.

A) Accuracy Group

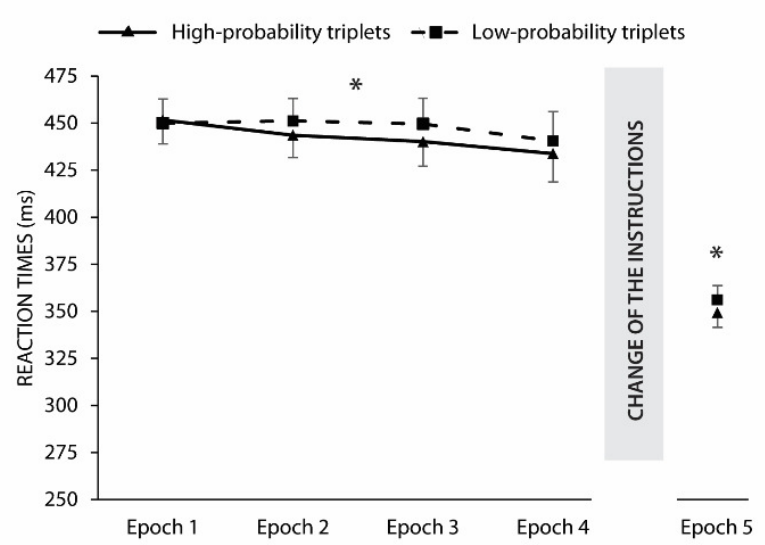

B) Speed Group

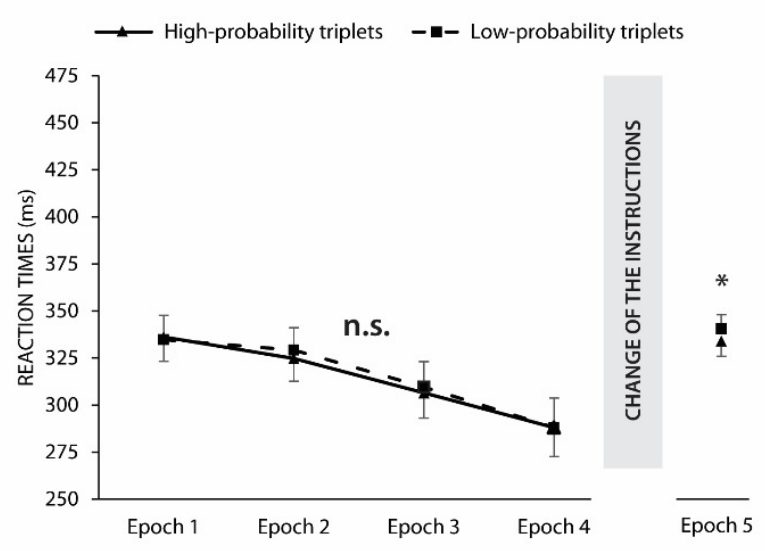

Supplementary Figure 1. Performance in the (A) Accuracy Group and (B) Speed Group when incorrect responses were also considered. The horizontal axis shows the five epochs of the task and the vertical axis the RTs. The solid line represents the RTs for the high-probability triplets, and the dashed line indicates the RTs for the lowprobability triplets. The error bars denote the standard error of the mean (SEM). Please note the gap between the two lines indicates the learning of statistical regularities. In the Same Instruction Phase (first four epochs), RTs for high- 
and low-probability triplets only differed in the Accuracy Group. However, a difference between the two trial types was measurable in both groups after the change of the instructions. $*=p<.05$, n.s. $=p>.05$

\section{Same Instruction Phase}

For the analysis of the Same Instruction Phase, we ran a mixed-design ANOVA with the within-subject factor of Triplet (high- vs. low-probability triplets) and the between-subjects factor of Group (Accuracy Group vs. Speed Group).

The main effect of Group was not significant, $F(1,59)=2.00, p=.16, \eta_{\mathrm{p}}^{2}=.03$, indicating that the speed difference disappeared after the change of the instructions; however, according to Bayesian comparison of the group means, the lack of difference was only anecdotal, $\mathrm{BF}_{01}=2.19$. The main effect of Triplet was significant, $F(1,59)=41.08 p<.001, \eta_{\mathrm{p}}{ }^{2}=.41$ : faster RTs were found for high-probability triplets compared to low-probability triplets $\left(\mathrm{BF}_{01}<0.001\right)$. The interaction between Triplet and the Group factors was non-significant, $F(1,59)=0.02, p=.90, \eta_{\mathrm{p}}{ }^{2}$ $<.001$ : despite the differences in the Different Instruction Phase, a comparable level of statistical knowledge was revealed after the change of the instructions $(p=.90)$. The Bayesian comparison of the difference also supported the null-hypothesis, $\mathrm{BF}_{01}=5.15$ (Supplementary Figure 2). We repeated the analyses with the standardized scores and again, no difference was found between groups, $t(59)=-0.07, p=.95, \mathrm{BF}_{01}=5.17$. 

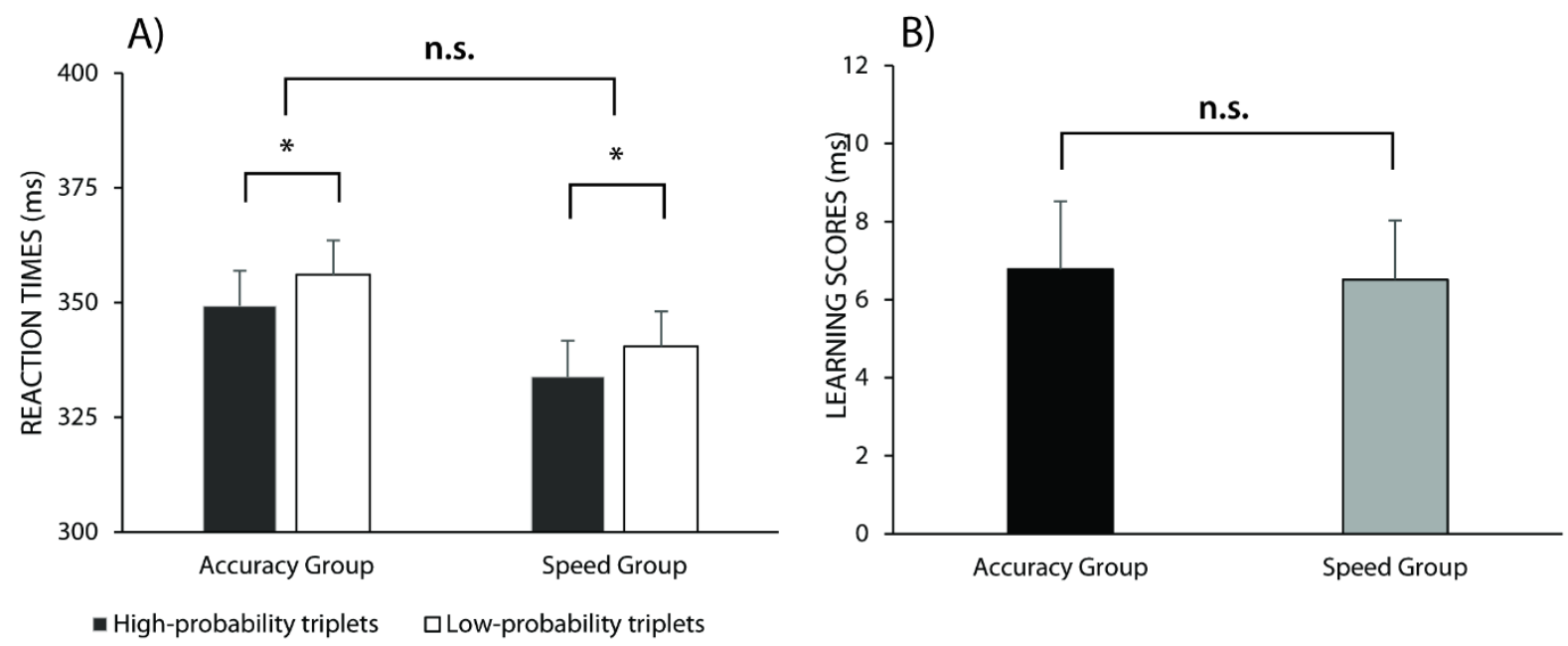

Supplementary Figure 2. Comparison of the high- and low-probability triplets (A) and learning scores (B) in the Same Instruction Phase (including incorrect responses). The vertical axis indicates the RTs (A) or the learning scores (RTs for low-probability triplets minus RTs for high-probability triplets, B), and the horizontal axis represents the two groups. The error bars denote the SEM. Although statistical knowledge was detected, no significant difference was found between groups, and the lack of difference was confirmed by Bayesian analysis. $*=p<.05$, n.s. $=p>.05$

\section{Bayesian Model Averaging in the Different Instruction Phase with the incorrect}

\section{responses included}

We conducted a Bayesian ANOVA on the difference between the two levels of the Triplet factor, (i.e., high-probability and low-probability triplets) with the within-subject factor of Epoch (Epoch 1-4) and the between-subject factor of Group (Accuracy Group vs. Speed Group). The Bayesian ANOVA supported the inclusion of the Epoch factor (Supplementary Table 7). The model tended to favor the exclusion of the interaction, and provided anecdotal evidence for the inclusion of the Group factor. This result suggests that the learning scores changed throughout the task, and this change was independent of the instructions (see detailed model comparisons in Supplementary Table 8). 


\section{Supplementary Table 7. Analysis of effects of the RT learning scores including errors}

\begin{tabular}{|c|c|c|c|}
\hline Effects & $\mathbf{P}($ incl $)$ & $\mathbf{P}($ incl|data) & BF excl \\
\hline Epoch & 0.60 & 0.97 & 0.05 \\
\hline Group & 0.60 & 0.64 & 0.85 \\
\hline Epoch $\times$ Group & 0.20 & 0.08 & 2.73 \\
\hline
\end{tabular}

Note: The column Effects indicates the main effects and interactions. The P(incl) column denotes the prior, and the $\mathrm{P}$ (incl|data) the posterior inclusion probability. The $\mathrm{BF}_{\text {exclusion }}$ column indicates the change from prior to posterior odds.

Supplementary Table 8. Bayesian model comparisons for RT learning scores including the errors

\begin{tabular}{lccccc}
\hline \multicolumn{1}{c}{ Models } & $\mathbf{P}(\mathbf{M})$ & $\mathbf{P}(\mathbf{M} \mid \mathbf{d a t a})$ & $\mathbf{B F}_{\mathbf{M}}$ & $\mathbf{B F}_{\mathbf{0 1}}$ & error \% \\
\hline Null model (incl. subject) & 0.20 & 0.01 & 0.05 & 1.00 & \\
Epoch + Group & 0.20 & 0.54 & 4.69 & 0.02 & 9.78 \\
Epoch & 0.20 & 0.35 & 2.13 & 0.04 & 1.26 \\
Epoch + Group + Epoch $\times$ Group & 0.20 & 0.08 & 0.37 & 0.15 & 3.72 \\
Group & 0.20 & 0.02 & 0.07 & 0.78 & 3.37 \\
\end{tabular}

Note. All models include subject. All models include subject. The Models column indicates the predictors included in each model, the $\mathrm{P}(\mathrm{M})$ column the prior model probability, the $\mathrm{P}(\mathrm{M} \mid$ data) column the posterior model probability, the $\mathrm{BF}_{\mathrm{M}}$ column the posterior model odds, and the $\mathrm{BF}_{01}$ column the Bayes factors of all models compared to the null model. The error is an estimate of the numerical error in the computation of the Bayes factor. 


\section{References}

Ellis PD. 2010. The Essential Guide to Effect Sizes, The Essential Guide to Effect Sizes. 41: $1581-1588$.

Faul F, Erdfelder E, Lang AG, Buchner A. 2007. G*Power 3: A flexible statistical power analysis program for the social, behavioral, and biomedical sciences. In: Behavior Research Methods. 39:175-191.

Nemeth D, Janacsek K, Fiser J. 2013. Age-dependent and coordinated shift in performance between implicit and explicit skill learning. Front Comput Neurosci. 7:147.

Nemeth D, Janacsek K, Király K, Londe Z, Németh K, Fazekas K, Adam I, Király E, Csányi A. 2013. Probabilistic sequence learning in mild cognitive impairment. Front Hum Neurosci. $7: 318$

Nemeth D, Janacsek K, Polner B, Kovacs ZA. 2013. Boosting human learning by hypnosis. Cereb Cortex. 23:801-805.

Virag M, Janacsek K, Horvath A, Bujdoso Z, Fabo D, Nemeth D. 2015. Competition between frontal lobe functions and implicit sequence learning: evidence from the long-term effects of alcohol. Exp Brain Res. 233:2081-2089. 